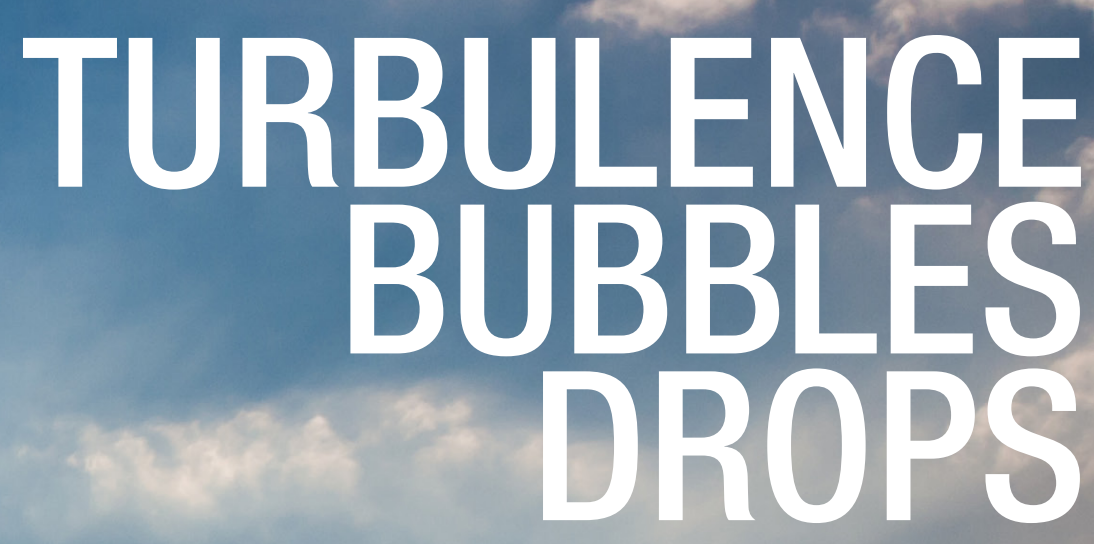

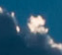
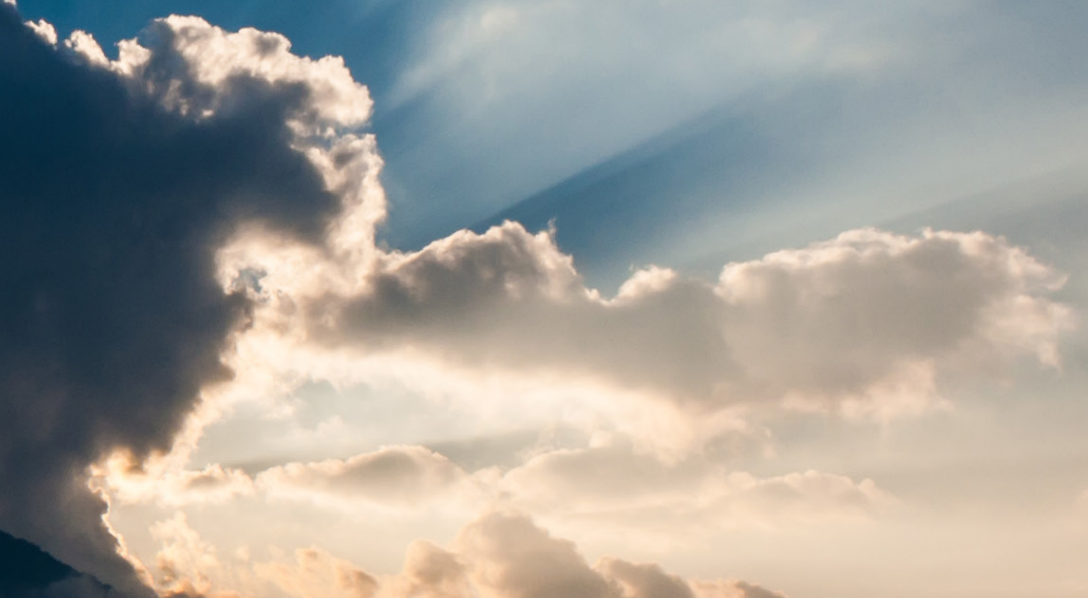

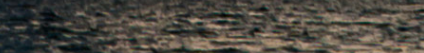




\section{TURBULENCE, BUBbLES AND DROPS}

Roeland Cornelis Adriaan van der Veen 


\section{Graduation committee members:}

Prof. dr. ir. Hans Hilgenkamp (chair) University of Twente

Prof. dr. rer. nat. Detlef Lohse (promotor) University of Twente

Prof. dr. Chao Sun (co-promotor) University of Twente

Prof. dr. Jennifer Herek University of Twente

Prof. dr. Roberto Verzicco University of Twente/Tor Vergata

Prof. dr. ir. Jerry Westerweel Delft University of Technology

Prof. dr. Ke-Qing Xia

Chinese University of Hong Kong

\section{European Research Council}

Established by the European Commission

The work in this thesis was carried out at the Physics of Fluids group of the Faculty of Science and Technology of the University of Twente. It was financially supported by a European Research Council Advanced Grant.

Dutch title: Turbulentie, bellen en druppels

Publisher:

Roeland C. A. van der Veen, Physics of Fluids, University of Twente, PO Box 217, 7500 AE Enschede, The Netherlands www.roelandvanderveen.nl

(C) 2016 Roeland C. A. van der Veen, Enschede, The Netherlands All rights reserved. No part of this publication may be reproduced by any means without the written permission of the publisher.

ISBN: 978-90-365-4083-4

DOI: $10.3990 / 1.9789036540834$ 


\title{
TURBUlENCE, BUBBLES AND DROPS
}

\author{
DISSERTATION
}

to obtain

the degree of doctor at the University of Twente, on the authority of the rector magnificus, prof. dr. H. Brinksma,

on account of the decision of the graduation committee, to be publicly defended

on Thursday the 24th of March 2016 at 16:45 hours

by

Roeland Cornelis Adriaan van der Veen

born on the 10th of August 1988

in Enschede, The Netherlands 
This dissertation has been approved by the promotor:

Prof. dr. rer. nat. Detlef Lohse

and the co-promotor:

Prof. dr. Chao Sun 


\section{Contents}

Introduction $\quad 1$

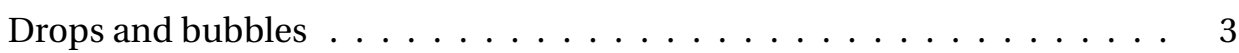

Taylor-Couette turbulence . . . . . . . . . . . . . . 6

I Drops and bubbles $\quad 11$

1 Direct measurements of air layer profiles under impacting droplets using $\begin{array}{ll}\text { high-speed color interferometry } & 13\end{array}$

1.1 Introduction $\ldots \ldots \ldots \ldots \ldots \ldots \ldots \ldots \ldots \ldots$

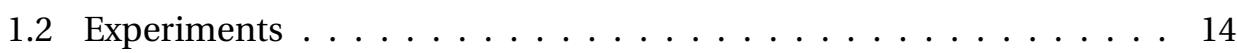

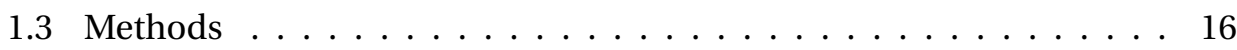

1.4 Results and discussion . . . . . . . . . . . . . . . . . 19

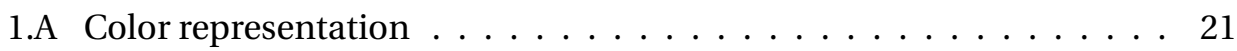

1.B A test case to assess the method's accuracy . . . . . . . . . 22

2 Maximal air bubble entrainment at liquid drop impact 25

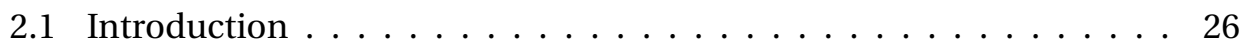

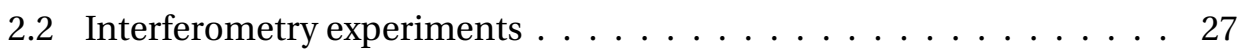

2.3 Boundary Integral simulations $\ldots \ldots \ldots \ldots \ldots$

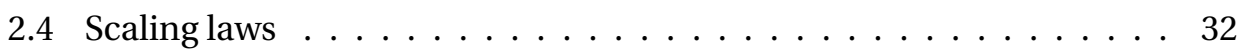

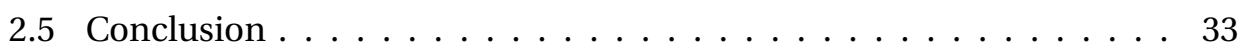

3 How microstructures affect air film dynamics prior to drop impact 35

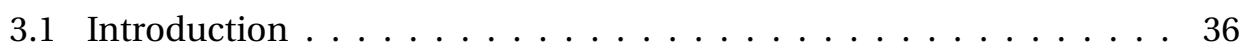

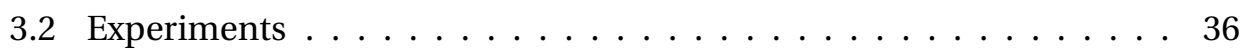

3.3 Results and discussion . . . . . . . . . . . . . . 38

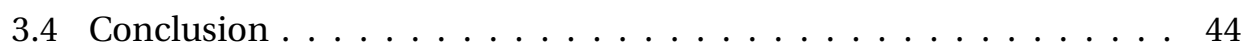

3.A Details on the method . . . . . . . . . . . . 44 
4 The Boiling Twente Taylor-Couette (BTTC) facility: temperature controlled turbulent flow between independently rotating, coaxial cylinders 49

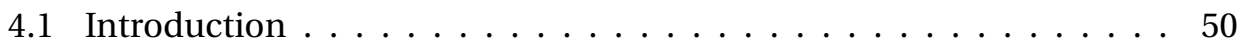

4.2 System description $\ldots \ldots \ldots \ldots \ldots \ldots$

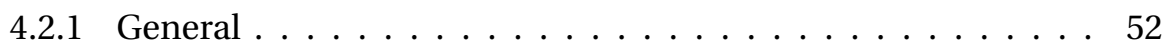

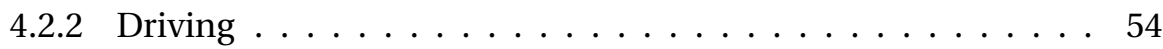

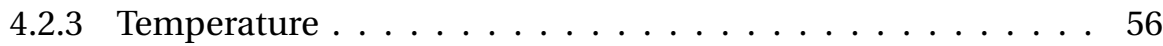

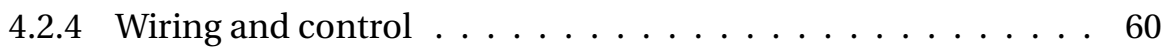

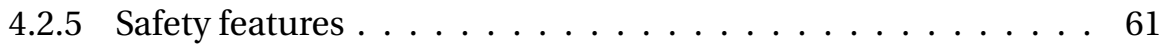

4.2.6 Other details and features $\ldots \ldots \ldots \ldots$

4.3 Example of flow measurement $\ldots \ldots \ldots$. . . . . . . . 63

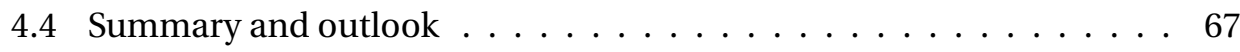

5 Multiple states in highly turbulent Taylor-Couette flow 69

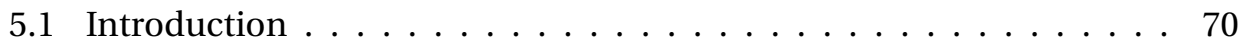

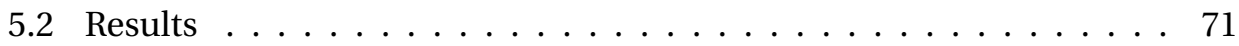

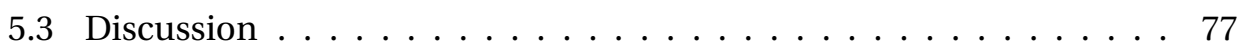

6 Exploring the phase space of multiple states in highly turbulent TaylorCouette flow $\quad 79$

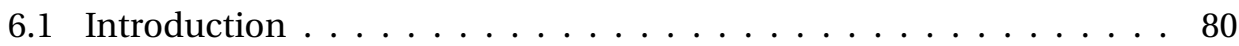

6.2 Setups and methods $\ldots \ldots \ldots \ldots \ldots$

6.3 Phase space of multiple states in the $\mathrm{T}^{3} \mathrm{C}$ with $\Gamma=11.7 \ldots \ldots$. . . 87

6.4 Flow structures in the BTTC setup with $\Gamma=18.3 \ldots \ldots \ldots \ldots$

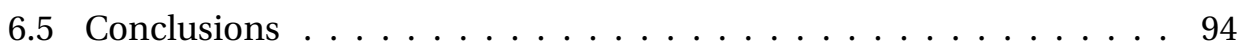

7 Taylor-Couette turbulence at radius ratio $\eta=0.5$ : scaling, flow structures and plumes $\quad 95$

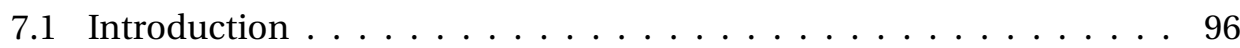

7.2 Setup \& explored parameter space $\ldots \ldots \ldots \ldots$

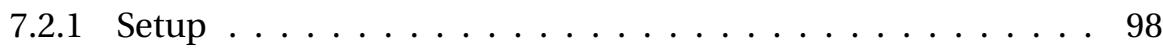

7.2 .2 Explored parameter space . . . . . . . . . . . . . . 99

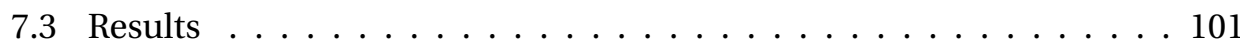

7.3.1 Azimuthal and angular velocity profiles . . . . . . . . . . . . . . . . . . . . . .

7.3.2 Wind Reynolds number . . . . . . . . . . . . . . . . . 103

7.3 .3 Roll structures . . . . . . . . . . . . . . . . . . . . . . . 105

7.3.4 Turbulent plumes . . . . . . . . . . . . . . . . . . . 107

7.3.5 Logarithmic velocity profiles . . . . . . . . . . . . . 111

7.4 Conclusions . . . . . . . . . . . . . . . . 113 
CONTENTS

Conclusions

Bibliography 119

Summary 131

Samenvatting 133

Acknowledgements 135

About the author 139

Publications 141 


\section{Introduction}

Turbulence, bubbles and drops. We are very familiar with these concepts from our daily lives. We know them from the awe-inspiring meteorological phenomena of storms and rain, to the trickling of the coffee machine.

Imagine a raindrop hitting a windshield, or a drop of ink hitting the paper in an inkjet printer. Perhaps surprisingly, these seemingly simple and mundane events of a falling drop hitting a surface can display a myriad of complicated phenomena, such as splashing, jetting, spreading, receding, rebounding, cavity formation and air entrainment. In the past century, huge steps have been taken in understanding these different drop impact events, although many are still not fully explained. In this thesis, the stage just before the drop fully hits the surface is investigated. It turns out that especially in this part of the impact process, the air surrounding the drop plays an important role, and that the deformation of the drop can result in an air bubble being entrapped below the drop. With a better understanding of this air entrainment phenomenon, it may be possible to optimize or improve many industrial and technical applications, such as spray painting, inkjet printing, sprinkling in agriculture, spray cooling and internal combustion.

Let us now turn our attention to the other side of the puzzle; turbulence. Just as drops and bubbles, turbulence can be found everywhere. Almost all fluid flows of human scale and larger are turbulent, from the breeze of a table fan to the convective flows in the sun. Turbulent flows exhibit irregular and chaotic behavior, and are therefore very hard to predict. The most sophisticated weather models running on the fastest supercomputers can only predict a few days into the future. To understand and predict turbulent flows in natural, industrial and technological applications, we need to study turbulence in a highly controlled way, on a more fundamental level. This is where model systems such as the Taylor-Couette (TC) setup come in. Taylor-Couette flow is the flow between two co-axial cylinders that can independently rotate. It is a fundamental configuration to test theories in fluid dynamics, and is very well suited to study turbulence. In this thesis various aspects of Taylor-Couette turbulence are studied, with the main focus on the existence of multiple turbulent states at very high Reynolds number. 


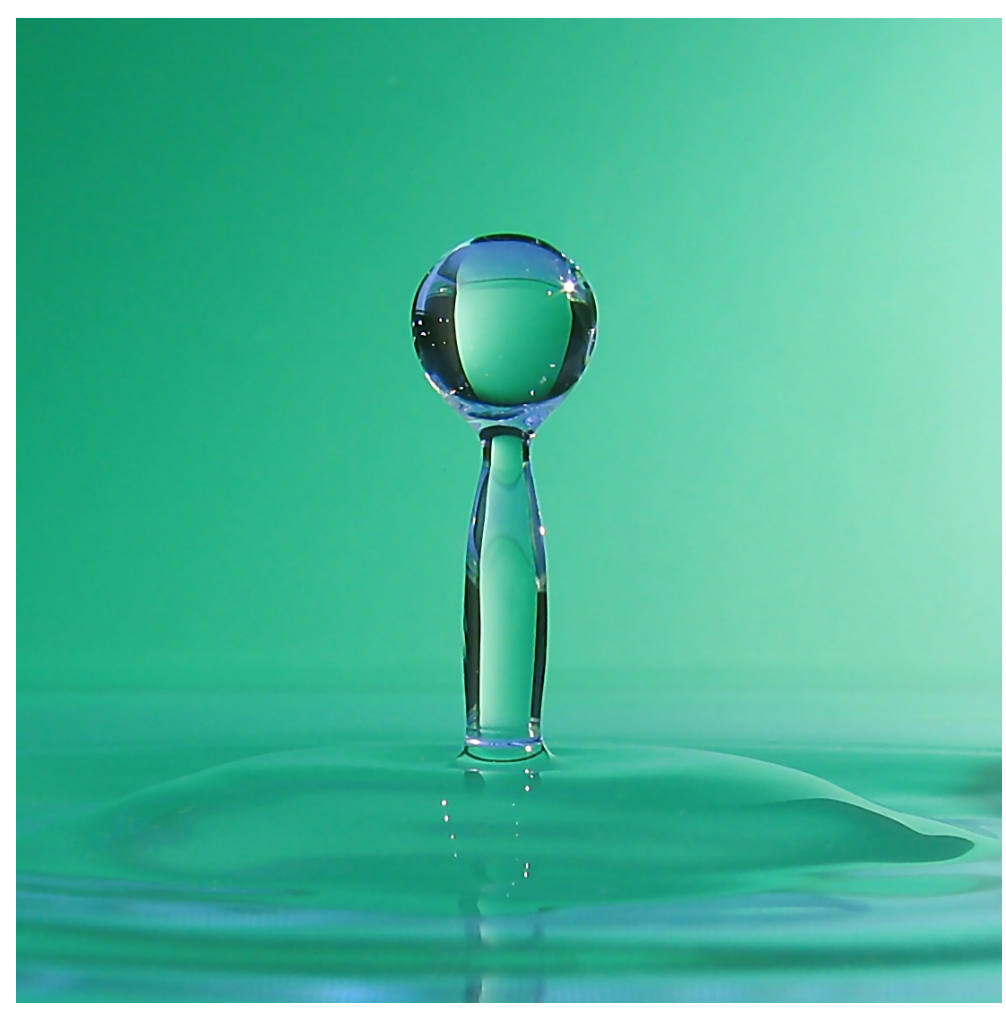

Figure 1: A jet created from a drop impacting on a liquid pool.

The connecting element between turbulence, bubbles and drops is, of course, the fluid itself. It is astounding that a single entity can display such a wide range of different phenomena, and, perhaps therefore, be of such great importance in our lives. Besides the fluid, another theme running throughout this thesis is 'visualization'. While the phenomena that we describe are familiar from daily life, the specifics often elude direct observation. For example, when turbulent flows contain only a single phase of fluid, the chaotic swirls and vortices cannot be observed directly. This is why measurements techniques such as particle image velocimetry (PIV) and laser Doppler anemometry (LDA) are used to reveal the behavior of fluid flows. In the case of drops, we are interested in what happens between the drop and the surface, on a sub-millimeter and sub-millisecond scale. Needless to say, also here, sophisticated measurement techniques are necessary to reveal the hidden phenomena. 


\section{Drops and bubbles}

\section{Fundamentals and applications}

The impact of drops exhibits a host of beautiful phenomena, such as the one shown in Fig. 1, where a drop impacting on a liquid pool creates a Worthington jet [1]. This is one of the many events of drop impact that are thoroughly treated in the reviews of Refs. [2-4]. In this thesis the focus will be on the impact of millimeter-sized drops on solid substrates with a range of impact velocities from millimeters per second to meters per second. In Fig. 2(a), a series of images is shown depicting the dynamics of drop impact. The drop first deforms before completely wetting the substrate. Before the wetting, an air film is present below the drop, which collapses into a bubble when contact occurs, see Fig. 2(b). This bubble is important for many applications; in the sense that often it is unwanted. For example, in inkjet printing it can reduce print quality, and in spray painting it can affect the finish of the paint. This is why it is important to know how the preceding air layer can be quantified, and how the bubble size can be controlled.

(a)
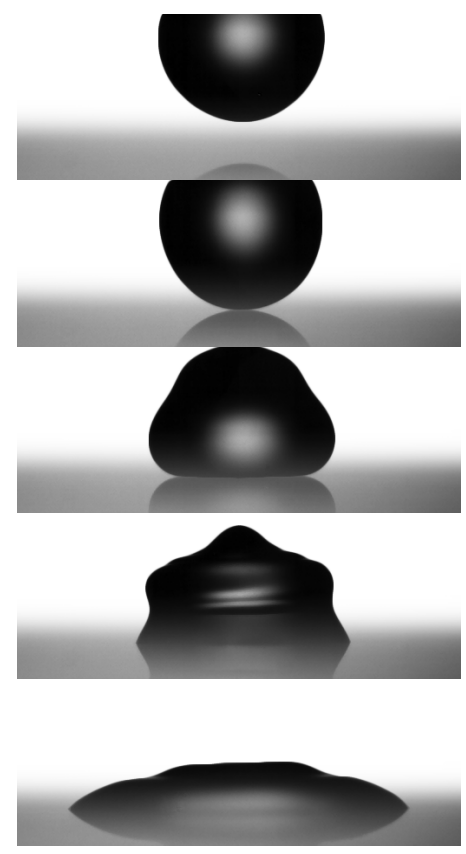

(b)
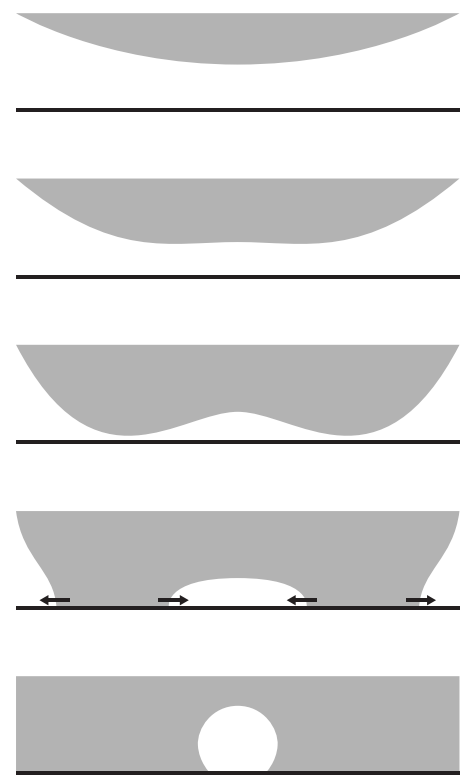

Figure 2: (a) Snapshots of a drop impacting on and wetting a surface. (b) Schematic of the bubble entrainment process. 


\section{Techniques}

\section{Interferometry}

The wavelength of visible light ranges from 390 to $700 \mathrm{~nm}$. While objects and structures of this size are impossible to directly resolve with human vision, effects can be seen in nature. When structures are of approximately the same size as the wavelength of light, they can make the light waves interfere, creating patterns with very specific colors. Examples are butterfly wings, soap bubbles and oil films on water (Fig. 3(a)). The oil film example has the most direct connection to the studies in this thesis, but instead of an oil film on water, we concern ourselves with the air layer between a drop and a surface, as sketched in Fig. 4(a). Light is sent in from below, which reflects from both the bottom of the drop and the top of the transparent substrate, and combines to create an interference pattern. One can imagine that the light source influences this interference pattern. In the case of a single-wavelength light source such as a laser, alternating dark and bright interference fringes as in Fig. 4(b) are observed. These, however, leave an ambiguity in the thickness of the film, because a change in thickness of an integer number of half wavelengths creates an identical pattern. This problem can be mitigated by using white light in combination with a color camera. This will create a pattern with colors that uniquely correspond to a certain film thickness, as in Fig. 4(c). This can be used to get an absolute film thickness of the air layer underneath impacting drops. In addition to using smooth surfaces, we also apply this color interferometry technique to transparent micropatterned surfaces to study impact on rough surfaces. A reconstruction of a fringe pattern created from a drop impacting on the latter kind of surface is shown in Fig. 3(b).

(a)

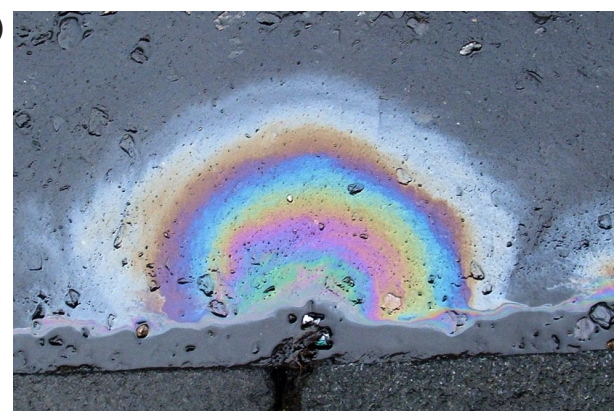

(b)

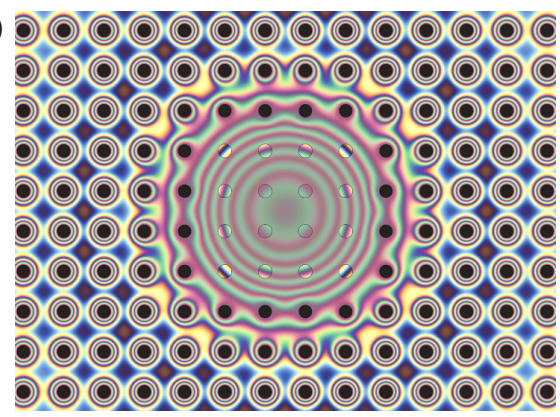

Figure 3: (a) Colorful interference pattern from an oil spill on a wet road. From https://commons.wikimedia.org/wiki/File:Dieselrainbow.jpg. (retrieved at 21-12-2015), by 'John' under the CC BY-SA 2.5 license. (b) Interference pattern created by light that reflects between a falling drop and a transparent microstructure. 
(a)

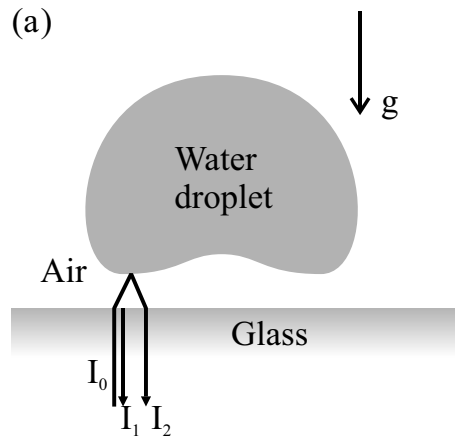

(b)

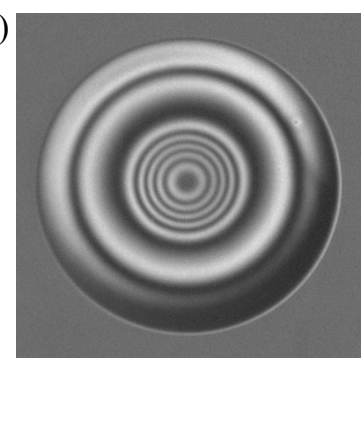

(c)

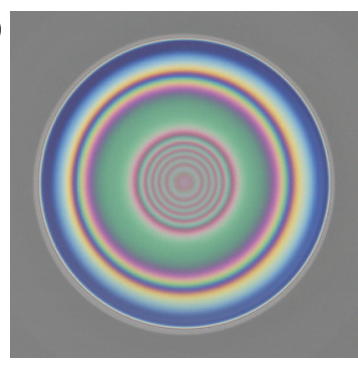

Figure 4: (a) Schematic view of a drop approaching the glass slide (not drawn to scale). Light is supplied from the bottom for illumination; reflection of light from the upper surface of the glass slide and from the bottom surface of the drop causes interference. (b) Interference pattern produced by a single-wavelength source, imaged by a grayscale camera. (c) Interference pattern produced by a broadband light source, imaged by a color camera.

\section{High-speed cameras}

In the past two decades, digital imaging has become all but indispensable for observing the physical behavior of fluids. Suppose one wants to image a drop moving over a distance of one millimeter at a velocity of one meter per second. When a sequence of ten images is required, a straightforward calculation shows that one needs to obtain 10,000 images per second. This lies far beyond the capabilities of ordinary video cameras, which record between 24 and 60 frames per second. We have to turn to high-speed cameras, which posses framerates from a few hundred frames per second, up to millions of frames per second. The reviews of Thoroddson et al. [5] and Versluis [6] treat the use of high-speed cameras in fluid dynamics. In this thesis the aforementioned color interferometry technique is combined with high-speed imaging to dynamically measure the air layer evolution underneath impacting drops. 


\section{Taylor-Couette turbulence}

\section{Fundamentals}

Taylor-Couette flow, named after Sir Geoffrey Ingram Taylor and Maurice Marie Alfred Couette, refers to the flow between two co-axial cylinders that can independently rotate [7-9]. It is one of the basic geometries in fluid dynamics, and draws interest from engineers, mathematicians and physicists. That is not surprising, since this setup can be mathematically well described and is experimentally easily accessible. Although the geometry is simple, the fluid flow within is not. Even for relatively slow rotation, the system shows very intricate flow behavior [10]. A large range of phenomena and concepts occur and have been studied in this system, such as instabilities, nonlinear dynamics and spatiotemporal chaos, pattern formation and turbulence. In this thesis the focus is on highly turbulent TC flow, which was recently reviewed by Grossmann, Lohse and Sun [11].

Although Taylor-Couette setups come in many shapes and sizes, the general geometry is captured in Fig. 5(a). Fig. 5(b) shows the $\mathrm{T}^{3} \mathrm{C}$ facility, which is one of the setups that is used in this thesis. The geometrical parameters of the TC system are the inner and outer cylinder radii $r_{i}$ and $r_{o}$ respectively, the gap width $d=r_{o}-$ $r_{i}$, and the height of the setup $L$. These can be expressed in dimensionless form by the radius ratio $\eta=r_{i} / r_{o}$ and the aspect ratio $\Gamma=L / d$. The inner and outer cylinder rotate with angular velocities $\omega_{i, o}$, which can be expressed in dimensionless form

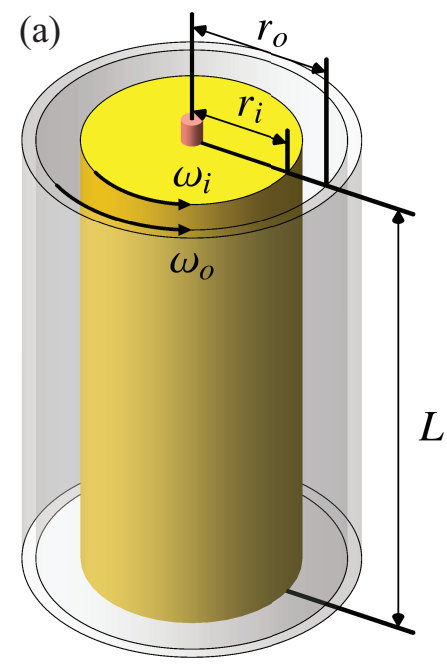

(b)

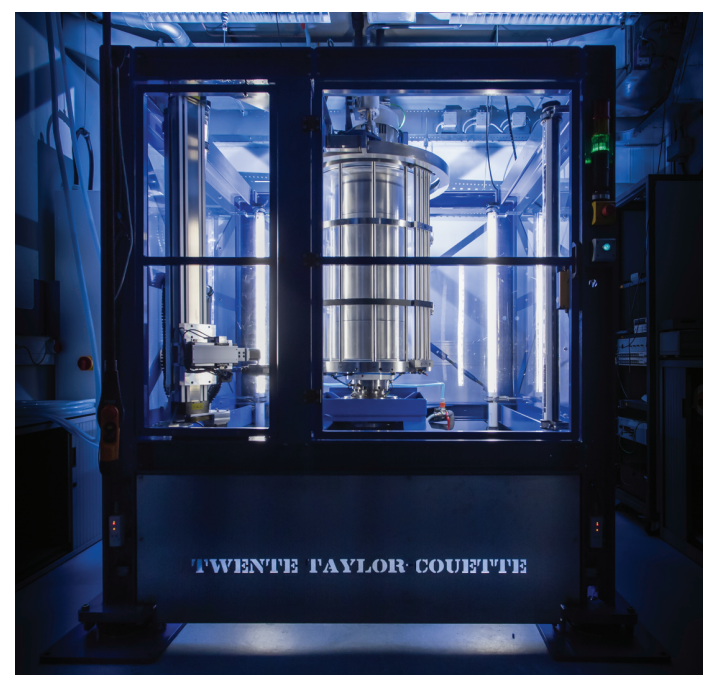

Figure 5: (a) Schematic of the Taylor-Couette setup with the main parameters. (b) The Twente Turbulent TC facility $\left(\mathrm{T}^{3} \mathrm{C}\right)$. 
by the Reynolds numbers $\operatorname{Re}_{i, o}=\omega_{i, o} r_{i, o} d / v$ where $v$ is the kinematic viscosity. Using the analogy [12] of TC flow with Rayleigh-Bénard convection, the driving of the flow can alternatively be characterized by the Taylor number:

$$
\mathrm{Ta}=\frac{(1+\eta)^{4}}{64 \eta^{2}} \frac{\left(r_{o}-r_{i}\right)^{2}\left(r_{i}+r_{o}\right)^{2}\left(\omega_{i}-\omega_{o}\right)^{2}}{v^{2}},
$$

combined with the (negative) rotation ratio

$$
a=-\frac{\omega_{o}}{\omega_{i}}
$$

with $a>0$ for counter-rotation, $a<0$ for corotation and $a=0$ for pure inner rotation. The response of the system is the torque $\tau$ required to sustain constant angular velocity or a 'Nusselt' number, $\mathrm{Nu}_{\omega}=\tau / \tau_{\text {laminar }}$, which is the angular velocity flux averaged over axial and azimuthal directions, nondimensionalised with the flux of the laminar, nonvortical, flow.

\section{Techniques}

However turbulent a flow is, when it consists of only a single phase fluid, no flow patterns will be visible. To study the flow patterns, we turn to laser-based measurement techniques such as particle image velocimetry (PIV) and laser Doppler anemometry (LDA). In contrast to techniques such as hot-wire anemometry, these are nonintrusive, meaning they do not affect the flow.

\section{LDA}

Laser Doppler anemometry (LDA), also known as laser Doppler velocimetry (LDV), uses the Doppler shift in a laser beam to measure the velocity in fluid flows. Typically, it consists of two laser beams intersecting at their waist, where they interfere and generate fringes. Particles in the fluid pass through the fringes and reflect light that is then collected by receiving optics and a photodetector. The reflected light fluctuates in intensity, the frequency of which is equivalent to the Doppler shift between the incident and scattered light, and is proportional to the particle velocity. Two or three pairs of beams can be used to measure two or three components of the velocity simultaneously. Although LDA is only a single-point measurement, it has the advantage that high data rates can be achieved. In addition, no calibration is necessary, which is a great advantage when measuring in e.g. the gap of a Taylor-Couette setup. In case one is interested in time-averaged quantities, it is possible to still obtain a velocity field with high spatial resolution by scanning the flow point-by-point. 

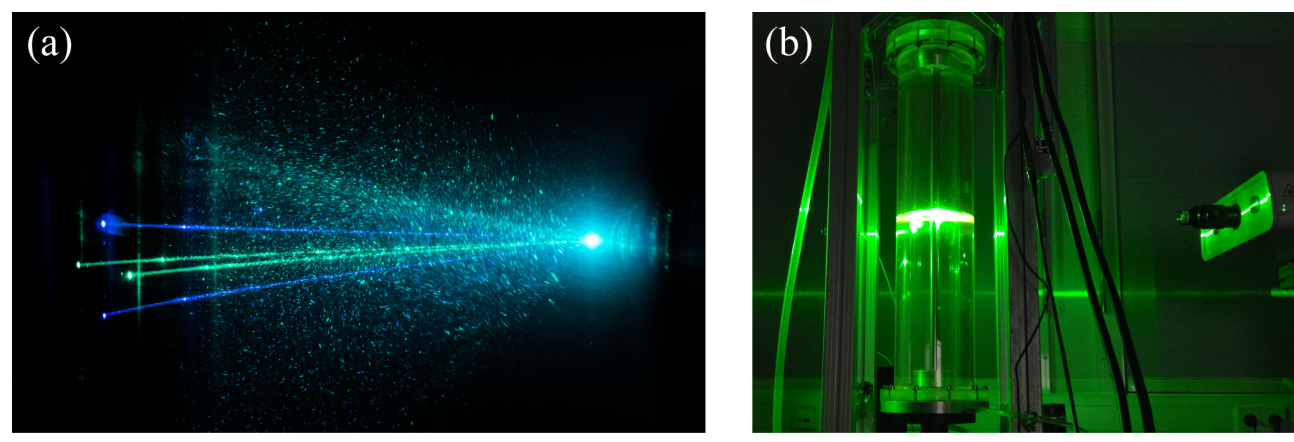

Figure 6: (a) The blue and green laser beams of an LDA setup. (b) PIV applied in a TaylorCouette setup.

\section{PIV}

Particle image velocimetry (PIV) makes it possible to measure the instantaneous velocity field simultaneously at many points, with a high spatial resolution. Most commonly it is used to extract snapshots of two- or three-component velocity vector fields on a planar cross section of the flow, although in recent years it has become possible to measure over volumetric domains and resolve the temporal evolution of the flow. A recent review is given by Westerweel et al. [13]. A typical modern PIV setup consists of a digital camera, a laser, synchronization hardware and the fluid with seeding particles. The laser gives off two pulses (with a predetermined time $\Delta t$ in between), which fall in two separate exposures of the camera. This results in two images with slightly displaced particles. Software partitions the images into blocks and performs signal processing to calculate displacement vectors for each block. Using the value of $\Delta t$ and the pixel size of the image, a velocity field can then be calculated. As compared to LDA, PIV offers the advantage of generating full, instantaneous velocity fields. When a fast sampling rate is desired, however, constraints with respect to the camera and data processing come into play. 


\section{Questions}

Drops and bubbles:

- How can the air layer dynamics underneath an impacting drop be measured? (chapter 1)

- How does the size of the entrapped air bubble depend on impact parameters? (chapter 2)

- How does the air layer behave in the case of impact on micropatterned surfaces? (chapter 3)

Taylor-Couette turbulence:

- Do large scale flow structures exist for highly turbulent TC flow, and if so, are multiple turbulent flow structures (states) possible? (chapter 5)

- For what parameter ranges do these multiple states exist? (chapter 6)

- Can the wind-Reynolds scaling that is predicted by Grossmann \& Lohse [14] be confirmed in the classical turbulent regime of TC flow? (chapter 7)

- Can turbulent plumes in TC flow be connected to the velocity profiles? (chapter 7)

\section{Guide through the thesis}

In Part 1 of the thesis, impacting drops and the role of the entrapped air are treated. A method to measure the air layer underneath an impacting drop is developed and tested in chapter 1 . In chapter 2 this method is applied to measure the entrapped bubble size for a large range of impact velocities. In chapter 3 the method is used to show the influence of microstructures on the deforming drop.

Part 2 of the thesis is about Taylor-Couette turbulence. In chapter 4 the development of a new Taylor-Couette setup is addressed. In chapter 5, the existence of multiple turbulent states in highly turbulent TC flow is investigated. The phase space of these multiple states is explored further in chapter 6 . In chapter 7 several properties of the turbulent flow in a $\eta=0.5$ TC setup are investigated, such as velocity profiles, turbulent plumes and scaling of the wind Reynolds number. 


\section{Part I}

\section{Drops and bubbles}





\section{Direct measurements of air layer profiles under impacting droplets using high-speed color inter- ferometry*}

A drop impacting on a solid surface deforms before the liquid makes contact with the surface. We directly measure the time evolution of the air layer profile under the droplet using high-speed color interferometry, obtaining the air layer thickness before and during the wetting process. Based on the time evolution of the extracted profiles obtained at multiple times, we measure the velocity of air exiting from the gap between the liquid and the solid, and account for the wetting mechanism and bubble entrapment. This chapter offers a tool to accurately measure the air layer profile and quantitatively study the impact dynamics at a short time scale before impact.

${ }^{*}$ Published as: Roeland C. A. van der Veen, Tuan Tran, Detlef Lohse and Chao Sun, Direct measurements of air layer profiles under impacting droplets using high-speed color interferometry, Phys. Rev. E 85, 026315 (2012). Development of the method, experiments, and analysis by RVDV, writing by TT and RVDV, supervision by CS and DL, discussion of the results and proofreading of the manuscript by everyone. 


\subsection{Introduction}

Drop impact on solid surfaces, beside its inherent beauty, has been playing an increasingly important role in industrial processes as diverse as ink-jet printing, spray cooling, and spray coating. Since it was first studied in 1876 by Worthington [15], the phenomenon has received tremendous attention from researchers, yet our understanding of this subject is still far from being complete (see review article [3]). A challenge in studying this problem arises from widely different time and spatial scales of the involved effects. Another difficulty comes from determining relevant physical parameters that govern the impact dynamics. For example, beside apparent parameters such as the surface roughness and wettability, the liquid viscosity, surface tension, and density, it was recently discovered that the ambient pressure is also a crucial parameter as it dictates the splash threshold after impact [16]. This finding and subsequent studies $[17,18]$ suggest that the air layer between an impinging droplet and a solid surface may have significant effects on the impact's outcomes. Hence, it is essential to understand how the drop and the surface interact through the air layer.

On the theoretical side, a mechanism of splash formation focusing on the short time scale within which the drop starts being deformed has been proposed [19, 20]. Detailed analysis and simulations have been subsequently developed [21, 22]. On the experimental side, the dynamics of droplet impact at the earliest time scale have also been studied; one of the most remarkable phenomena is the detection of entrapped bubbles under an impacting drop [23-26]. The existence of these bubbles indicates that the drop's bottom surface is deformed before it makes contact with the surface. There is, however, a lack of detailed measurements of the air layer thickness at the earliest time of impact, as well as the formation of the entrapped bubbles.

Here we report the first direct measurement of the evolution of the air layer profile between an impinging droplet and a solid surface using high-speed color interferometry. We focus on the earliest time of impact when the liquid has not touched the surface but starts being deformed due to the pressure increase in the air layer between the liquid and the solid surface. We measure the air flow between the droplet and the solid surface, and investigate the mechanism of bubble entrapment.

\subsection{Experiments}

In Fig. 1.1(a), we show a schematic of the experimental setup for the present work. We generate liquid drops by using a syringe pump to push liquid out of a fine needle. The drop detaches as soon as its weight overcomes the surface tension and 

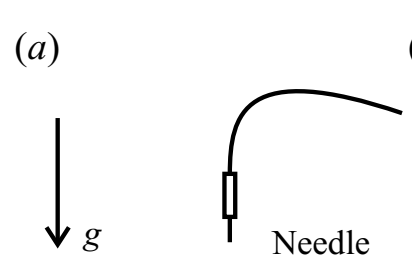

(b)
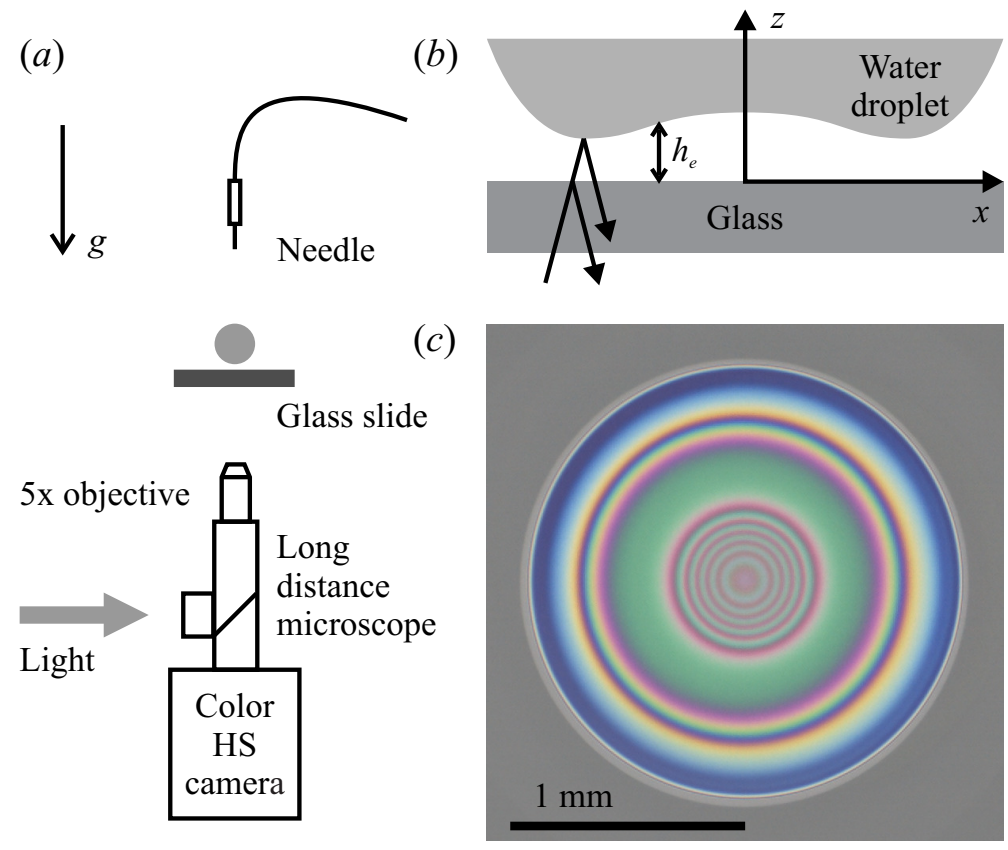

Figure 1.1: (a) Schematic of the experimental setup (not drawn to scale) used to study droplet impact on smooth surfaces. A water droplet of initial diameter $D_{0} \approx 2 \mathrm{~mm}$ falls on a glass slide of average roughness $10 \mathrm{~nm}$. The bottom view is captured by a high-speed color camera (SA2, Photron Inc.). The camera is connected to a long working-distance microscope and a $5 \mathrm{x}$ objective to obtain a $2 \mathrm{~mm}$ field of view. (b) Schematic of the air film between the drop and the glass slide (not drawn to scale). Light is supplied from the bottom for illumination; reflection of light from the upper surface of the glass slide and from the bottom surface of the drop causes interference fringes captured with the color camera. (c) An example of an interference pattern.

then falls on a microscope glass slide (Menzel microscope slide, average roughness $\approx 10 \mathrm{~nm}$ ). In our experiments, the working liquid is milli-Q water (density $\rho_{w}=998 \mathrm{~kg} / \mathrm{m}^{3}$, surface tension $\sigma_{w}=72 \times 10^{-3} \mathrm{~N} / \mathrm{m}$, viscosity $v_{w}=10^{-6} \mathrm{~m}^{2} / \mathrm{s}$ ). The drop typically has diameter $D \approx 2 \mathrm{~mm}$ and its velocity before impacting the surface can be adjusted by varying the needle's height $H$. We capture the drop impact from the bottom with a color high-speed camera (SA2, Photron Inc.) connected to a long working-distance microscope (Navitar Inc.) and a 5X objective. The field of view achieved by this combination is $2 \mathrm{~mm}$. We illuminate the impact area from below by supplying white light from a high-intensity fibre lamp (Olympus ILP-1) to the microscope's coaxial light port. When a drop approaches the glass slide, a thin film of air is formed between the liquid and solid surfaces before wetting occurs. Light of the same wavelength coming from the bottom, 


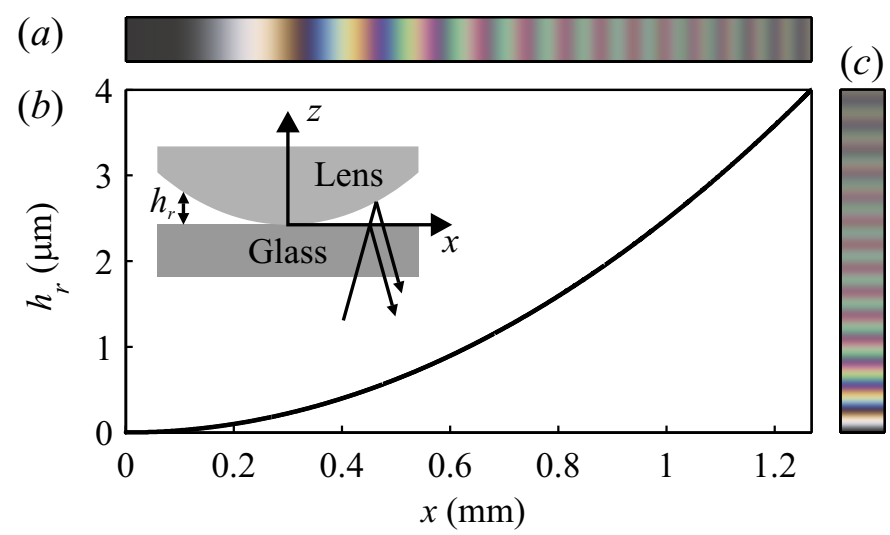

Figure 1.2: (a) Color variation in the radial direction of the interference pattern used to calibrate colors. (b) Reference thickness of the air film between a lens and a glass slide. The lens has radius of the surface in adjacent with the glass $R=200 \mathrm{~mm}$. Inset: schematic of the setup used to calibrate colors. (c) Relation between thickness and reference colors.

upon reflection from both surfaces of the film (Fig. 1.1(b)) forms interference patterns recorded by the camera. Each one of these patterns consists of constructive (bright) and destructive (dark) fringes; the fringe spacing depends on the air layer thickness and the wavelength of incident light. Since the lamp emits light of multiple wavelengths, the superposition of all available patterns produces concentric rings of rainbow colors as shown in Fig. 1.1(c).

In most of our experiments, we set the camera's frame rate to 10000 frames per second (FPS), and its resolution to $512 \times 512$ pixels to capture droplets with impact velocity less than $0.5 \mathrm{~m} / \mathrm{s}$. In the case that the impact velocity is higher, the frame rate can be set as high as 86400 FPS at resolution $32 \times 256$ pixels to capture the impact dynamics.

\subsection{Methods}

In order to extract the absolute thickness of the air layer between an impinging drop and a glass surface, we construct a set of reference colors that can be related to absolute thickness. We put a convex lens on top of the glass slide (see inset in Fig. 1.2(b)) and observe the interference rings caused by the air film between two surfaces. Since the pattern consists of concentric rings of different colors, and the air film thickness is known at each radial location, each color along a line passing through the center of these rings is associated with a thickness value. In Fig. 1.2(b), we show the air thickness profile between the lens and the glass slide. The color variation due to change in air thickness is obtained by taking a thin radial strip 
of $100 \times 2200$ pixels from an image of an interference pattern and then averaging colors in the transverse direction to reduce noise. The resulting strip (Fig. 1.2(a)), which has no color variation in the transverse direction, contains $N=2200$ pixels in the $x$-direction and hence $N$ reference colors that can be used for calibration. Since the camera uses the sRGB model to represent colors, the color of each pixel $i$ is represented by a color vector $\left(R_{i}, G_{i}, B_{i}\right)$. The pixel's coordinate is $x_{i}$, which is related to a value of thickness $h_{r}^{i}$. Thus, we have a set of reference colors $\left(R_{i}, G_{i}, B_{i}\right)$ for $1 \leq i \leq N$, each of which is associated with a reference thickness $h_{r}^{i}$. The reference thickness range is $0 \leq h_{r}^{i} \leq 4 \mu \mathrm{m}$. The thickness-color relation is shown in Fig. 1.2(c).

The sRGB model, however, is generally not preferred when comparing colors between experiments because it does not decouple light intensity and color information, which poses a problem due to variations in illumination conditions such as light intensity, incident and observing angles. Instead, we use the CIE 1976 color model (also called CIELAB), a model that is most effective in decoupling light intensity [27] (see appendix 1.A for details). To work with colors in the CIELAB color space, we convert sRGB-format images to the absolute color space (XYZ) and then to CIELAB [27]. A color in CIELAB model has three components: $\boldsymbol{L}$ for lightness information, and $\boldsymbol{a}$ and $\boldsymbol{b}$ for color information. Thus, we can separate light intensity from our analysis by omitting the component $\boldsymbol{L}$. Each reference color $i$ after intensity decoupling is represented by a two-component vector $\left(\boldsymbol{a}_{r}^{i}, \boldsymbol{b}_{r}^{i}\right)$ and is associated with a value of reference thickness $h_{r}^{i}$ for $1 \leq i \leq N$ and $h_{r}^{i}$ is in the range $0 \leq h_{r}^{i} \leq 4 \mu \mathrm{m}$.

In Fig. 1.3(a), we show a color sample, which was taken along a diameter of an interference pattern under a drop with impacting velocity $V=0.22 \mathrm{~m} / \mathrm{s}$. After converting the color of each pixel to CIELAB color space and decoupling light intensity, we calculated the color difference $d E^{i j}$ for each color $\left(\boldsymbol{a}_{e}^{j}, \boldsymbol{b}_{e}^{j}\right)$ in the sample $(1 \leq j \leq M=596)$ and each reference color $\left(\boldsymbol{a}_{r}^{i}, \boldsymbol{b}_{r}^{i}\right)$ using the Euclidean distance:

$$
d E^{i j}=\left[\left(\boldsymbol{a}_{e}^{j}-\boldsymbol{a}_{r}^{i}\right)^{2}+\left(\boldsymbol{b}_{e}^{j}-\boldsymbol{b}_{r}^{i}\right)^{2}\right]^{1 / 2},
$$

for $1 \leq i \leq N$ and $1 \leq j \leq M$. Since each color $\left(\boldsymbol{a}_{e}^{j}, \boldsymbol{b}_{e}^{j}\right)$ is associated with a coordinate $x_{j}$ and, recall that each reference color $\left(\boldsymbol{a}_{r}^{i}, \boldsymbol{b}_{r}^{i}\right)$ is associated with a value of reference thickness $h_{r}^{i}$, the color difference $d E^{i j}$ can be thought of as a function of $h_{r}^{i}$ and $x_{j}$. In Fig. 1.3(b), we show a plot of $d E^{i j}$ in grayscale for $1 \leq i \leq N$ and for $1 \leq j \leq M$. The range of the index $i$ translates to the range of reference thickness $0 \leq h_{r} \leq 4 \mu \mathrm{m}$, and similarly that of the index $j$ to $-1 \leq x \leq 1 \mathrm{~mm}$. In the plot, black means $d E=0$ and hence zero color difference, whereas white means the largest color difference. A vertical line at a particular value of $x$ has all possible values of the film thickness at that point; the correct thickness value corresponds to the darkest point. In the case that there are multiple dark points on the same 


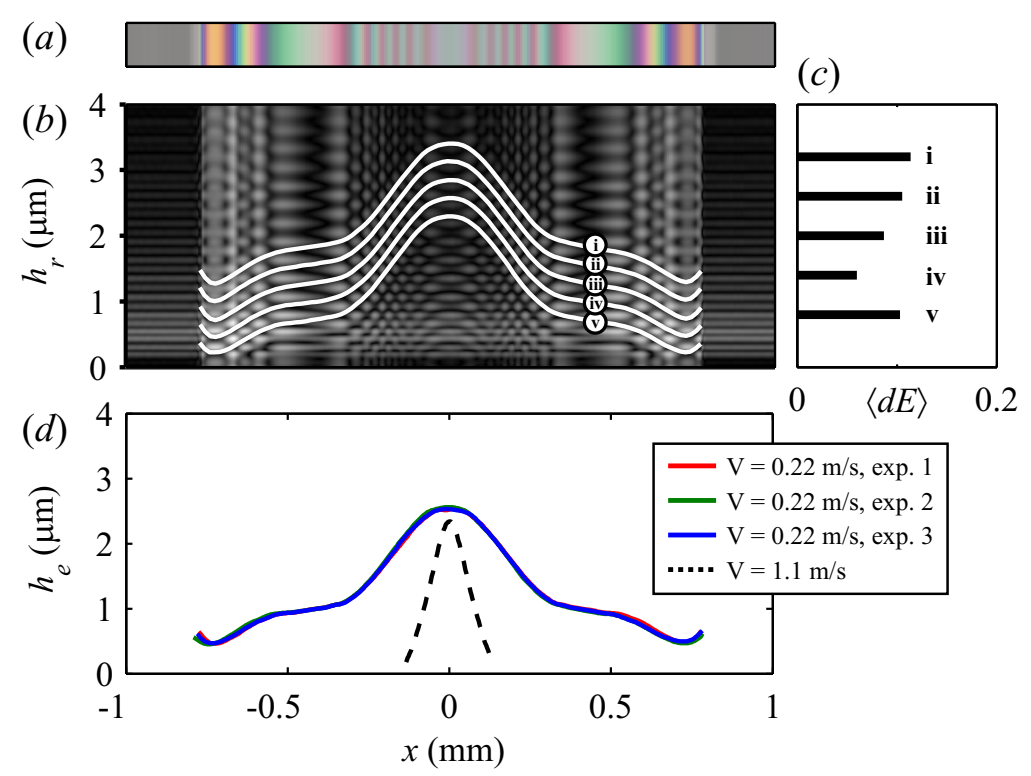

Figure 1.3: (a) Color sample of interference pattern taken at $t=1.67 \mathrm{~ms}$ after the bottomview camera detected the drop. (b) Color difference in grayscale computed by Eq. 1.1 with candidate profiles shown in white solid lines. (c) Average color difference of candidate profiles shown in $(b)$. (d) Solid lines: profiles computed from three different experiments with $V=0.22 \mathrm{~m} / \mathrm{s}$. Dashed line: $V=1.1 \mathrm{~m} / \mathrm{s}$. Note the extremely different length scales at the $x$-axis $(\mathrm{mm})$ and $h_{r}$-axis $(\mu \mathrm{m})$ in $(b)$ and $(d)$.

vertical line with insignificant difference between them, thickness determination is not trivial (see appendix 1.A for details). We note that, however, the film profile is continuous and smooth. Evidently, there are only a few continuous dark lines that can be distinguished without any abrupt change in slope. In fFig. 1.3(b), we show the candidate profiles in white solid lines (labeled from (i) to (v)). The film thickness profile can be identified by considering the average color difference $\langle d E\rangle$ along each candidate profile $L$ :

$$
\langle d E\rangle^{L}=\frac{1}{N_{L}} \sum_{L} d E^{L},
$$

where the sum is taken for all the pixels along the profile $L$ and then divided by the number of pixels $\left(N_{L}\right)$. In Fig. 1.3(c), we show $\langle d E\rangle^{L}$ for all profiles. The smallest color difference is along profile $(i v)$ for which $\langle d E\rangle^{i v}=0.06$, whereas the second smallest one is along profile $(i i i)$ for which $\langle d E\rangle^{i i i}=0.09$. As a result, we conclude that profile $(i v)$ is the air layer profile. A test case of an air film with a known thickness profile shows that the accuracy of our method is within $40 \mathrm{~nm}$ (see appendix 1.B). To check the reproducibility we repeated the experiment several times and 

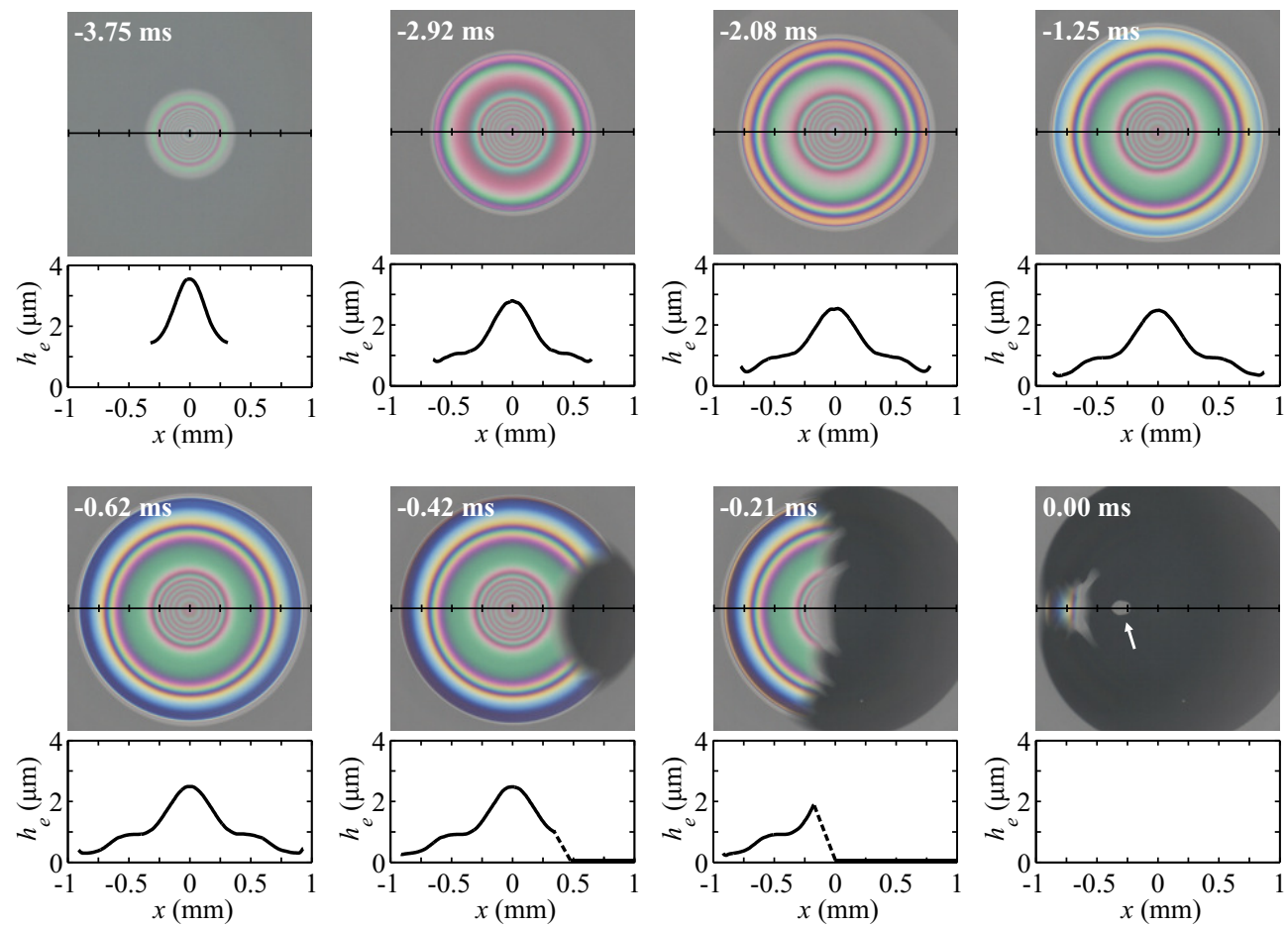

Figure 1.4: Snapshots of interference patterns obtained during drop impact and their corresponding calculated profiles $(V=0.22 \mathrm{~m} / \mathrm{s}, D=2 \mathrm{~mm})$.

extracted the air thickness in each experiment at the same time. The computed profiles are shown in Fig. 1.3(d). Given the variations between experiments such as releasing time, drop size, surface properties, etc., the method gives remarkably consistent results.

\subsection{Results and discussion}

In Fig. 1.4, we show interference patterns obtained during drop impact $(V=0.22$ $\mathrm{m} / \mathrm{s}$ and $D=2 \mathrm{~mm}$ ) and their corresponding thickness profiles of the air layer. We define $t=0$ as the moment when the liquid completely wets the solid surface. From the first pattern detected by the camera $(t=-3.75 \mathrm{~ms})$, it is readily seen that a dimple is already formed, which means that the camera did not capture the entire deformation process of the drop's lower surface, probably due to limited coherence length of the light source used in the present experiment. Subsequent profiles show that the dimple's height gradually reduces, while the liquid continues spreading in the radial direction. At $t=-0.42 \mathrm{~ms}$, the liquid starts wetting the glass surface at one point along the rim of the air layer and then propagates to the 

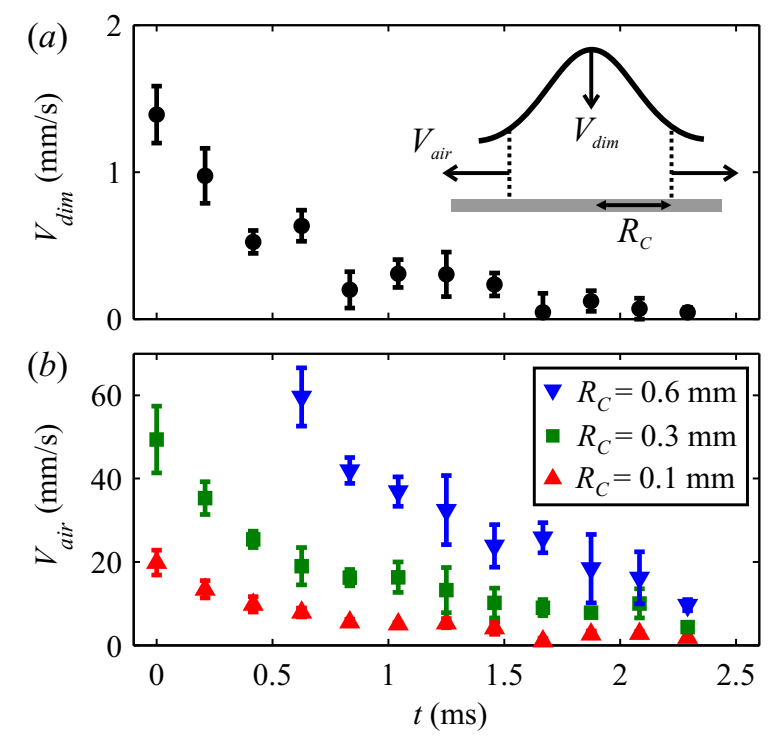

Figure 1.5: (a) Dimple velocity $V_{d i m}$ vs. time. (b) The average velocity of air $V_{a i r}$ vs. time at different radial locations $R_{C}=0.1 \mathrm{~mm}$ (upward triangles), $R_{C}=0.3 \mathrm{~mm}$ (squares), $R_{C}=$ $0.6 \mathrm{~mm}$ (downward tringles) for an experiment with impact velocity $V=0.22 \mathrm{~m} / \mathrm{s}$.

other side; the wetting process happens faster along the rim where the air thickness is smallest and finally traps air bubbles (indicated by an arrow at $t=0 \mathrm{~ms}$ ). For experiments done under the same conditions, although the time that wetting occurs varies, we observe the same bubble-trapping dynamics, that is, the wetting front propagates faster at the rim and finally encloses the air pocket underneath the drop.

We note that there is a plateau (at $x \approx \pm 0.5 \mathrm{~mm}$ ) in the thickness profiles from $t=-2.92 \mathrm{~ms}$ to $t=-0.21 \mathrm{~ms}$. This is due to the drop's oscillation as it falls down at a small distance from the surface (in this case $H=6 \mathrm{~mm}$ ). When the drop detaches from the needle, capillary waves are generated and propagate to the other side. The surface deformation caused by these waves affects the dimple shape in addition to the pressure increase in the air layer under the drop. In the case that the drop is released from a larger height leaving sufficient time for viscosity to damp capillary waves, we do not observe the plateau in the thickness profiles. As shown in Fig. 1.3(d), the plateau is not present in the case of higher impact velocity case $(V=1.1 \mathrm{~m} / \mathrm{s}, H=66 \mathrm{~mm})$.

We now quantify the velocity at the center of the dimple $V_{\text {dim }}$ (see inset of Fig. 1.5(a)). As shown in Fig. 1.5(a), $V_{\text {dim }}$ is found to be very small (roughly two orders of magnitude smaller) as compared to the impact velocity $V=0.22 \mathrm{~m} / \mathrm{s}$, which implies that the fluid at the bottom of the drop has decelerated before the camera starts capturing the interference fringes. Nonetheless, our measurements 
capture well the deceleration process of the lower surface of the drop from the detection point until it is brought to rest. Moreover, we estimate the horizontal velocity of air $V_{a i r}$ based on the change in volume confined by a cylinder of radius $R_{C}$ under the liquid surface (inset of Fig. 1.5(a)). In Fig. 1.5(b), we show $V_{a i r}$ at several values of $R_{C}$. The data show a consistent increase of the air velocity at a given time as it gets closer to the rim of the air layer where the thickness is minimum. For higher impact velocity cases, the velocity of air is much higher due to the extremely thin air gap at the rim.

In conclusion, we have used high-speed color interferometry to measure the complete profile and its evolution of the air layer under an impacting drop for impact velocity $V=0.22 \mathrm{~m} / \mathrm{s}$ and $V=1.1 \mathrm{~m} / \mathrm{s}$. From the experimental measurements, we account for the wetting mechanism which results in entrapment of bubbles after impact. We also experimentally quantify the velocity of air flow between the drop and the surface, as well as the velocity of the dimple before wetting occurs. Our results offer a benchmark for theories of drop impact.

\section{A Color representation}

The necessity of decoupling light intensity from color analysis leads us to resource the CIELAB model instead of the sRGB one. Here we present a test case comparing sRGB and CIELAB models. First we reduce light intensity in the interference pattern resulting from the calibration step by multiplying each channel of the RGB model by 0.75 . The darkened pattern is shown in Fig. 1.6(a). We use this pattern as a color sample from which air layer thickness is recovered. An image showing the color difference using sRGB representation in grayscale between the modified color set and the reference one is shown in Fig. 1.6(b). Clearly, without intensity decoupling, it is difficult to recover the air thickness profile. Even examining the average color difference along a few candidate profiles does not reveal which one is correct (Fig. 1.6(d)). In contrast, the color sample after intensity decoupling with the CIELAB model gives profiles with high contrast from the background (see Fig. 1.6(e)). Fig. 1.6(g) also shows that the correct one also has the smallest value of average color difference along candidate profiles in Fig. 1.6(f).

We now discuss an inherent issue of methods using color interferometry to measure film thickness regardless of color model, namely, repetition of colors at multiple values of film thickness [28]. We demonstrate this problem for the reference colors represented by the CIELAB model in Fig. 1.7(a). The plot shows $\boldsymbol{b}_{r}^{i}$ vs. $\boldsymbol{a}_{r}^{i}$ for $1 \leq i \leq N$ and correspondingly $0 \leq h_{r}^{i} \leq 4 \mu \mathrm{m}$. Note that each pair of $\left(\boldsymbol{a}_{r}^{i}, \boldsymbol{b}_{r}^{i}\right)$ represents one color and is associated with a value of thickness $h_{r}^{i}$. Thus, at each intersection of the curve with itself, there are two values of thickness producing the same color in the interference pattern. By omitting these points, we obtain 
(a)

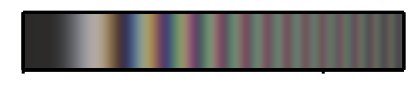

0 $x(\mathrm{~mm}) \quad 1$
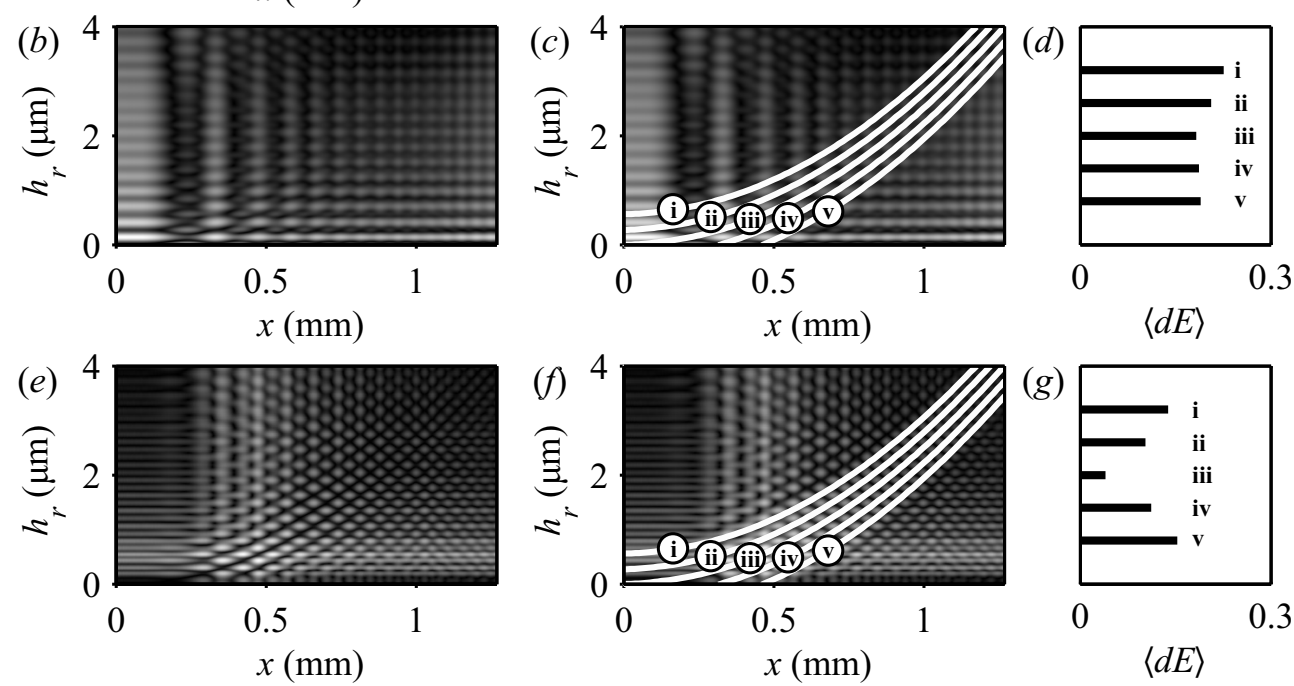

Figure 1.6: (a) Color variation (after reducing the intensity to 75\%) depending on the air film thickness obtained in the calibration step. $(b)$ sRGB model: color difference between the reference colors and the darkened ones in grayscale. (c) Candidate profiles resulted from the color difference in $(b)$. (d) The averaged color differences along the candidate profiles shown in $(c)$. (e) CIELAB model: color difference between the reference colors and the darkened ones in grayscale. ( $f$ ) Candidate profiles resulted from the color difference in $(e)$. $(g)$ The averaged color differences along the candidate profiles shown in $(f)$.

the thickness values that produce unique colors (Fig. 1.7(b)). It is clearly seen that the color database can be used to estimate film thickness ranging from $0.5 \mu \mathrm{m}$ to $1 \mu \mathrm{m}$ without ambiguity. Outside of this range, thickness measurements for individual points are not reliable. The entire profile, however, can be constructed if the smoothness and continuity of thickness profiles are taken into account.

\section{B A test case to assess the method's accuracy}

First, we generate an interference pattern from an air film between a glass slide and a lens. This arrangement is similar to the one used for color calibration but with a different lens (the radius of the lens used in this setup is $300 \mathrm{~mm}$ ). From the resulting interference pattern, we exclude the part where two surfaces are in contact (correspondingly, the color is close to black) to simulate the real situations in which the liquid does not necessarily touch the solid surface. The film thickness that needs to be determined is within the range of the calibrated thickness. The 

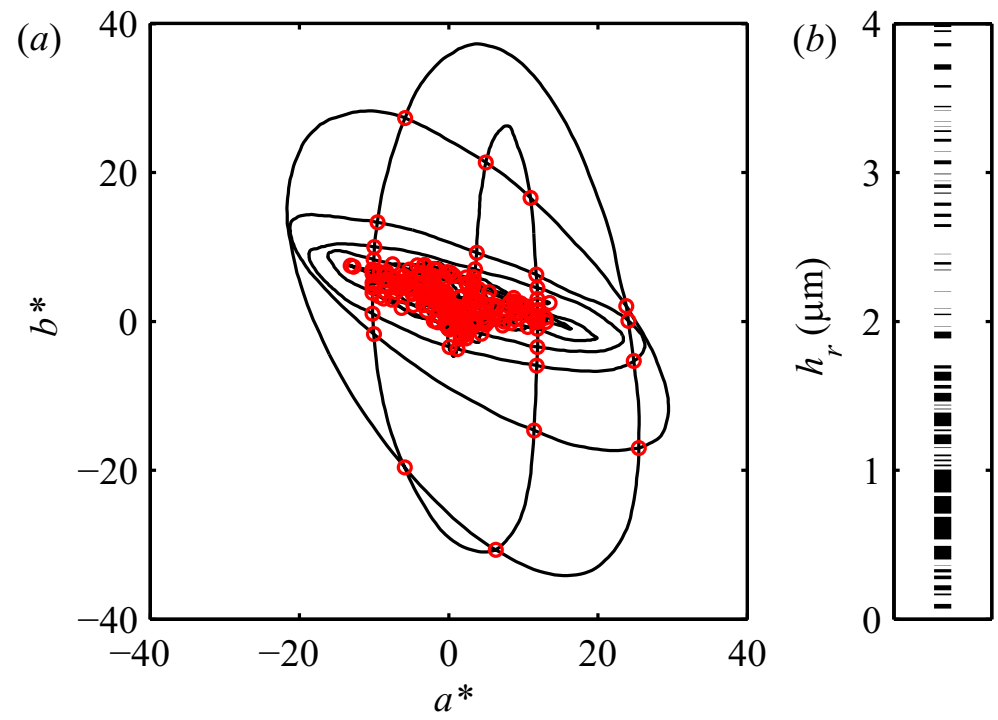

Figure 1.7: (a) Plot of reference color in $\boldsymbol{a}_{r}, \boldsymbol{b}_{r}$ space showing the color is not unique at some thickness values (red circles). (b) Only thickness values that have unique color are shown.

interference pattern, after average azimuthally and expanded in the transverse direction, is the color sample shown in Fig. 1.8(a).

Using the method described in Sec. 1.3, we decouple light intensity from the color sample and construct a grayscale plot of color difference between reference and sampled colors. The grayscale plot with candidate profiles of the air thickness are shown in Fig. 1.8(b). The profile with the smallest average color difference is then plotted against the profile of the lens used to generate the interference pattern (Fig. 1.8(d)). As can be seen the difference between these profiles is no greater than $40 \mathrm{~nm}$. 


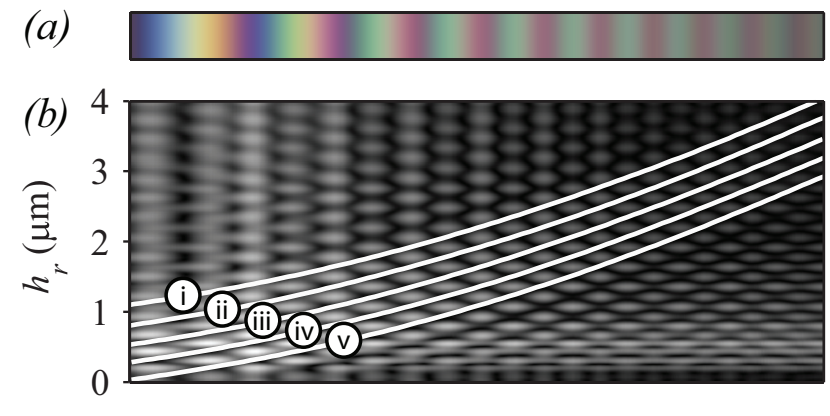

(c)
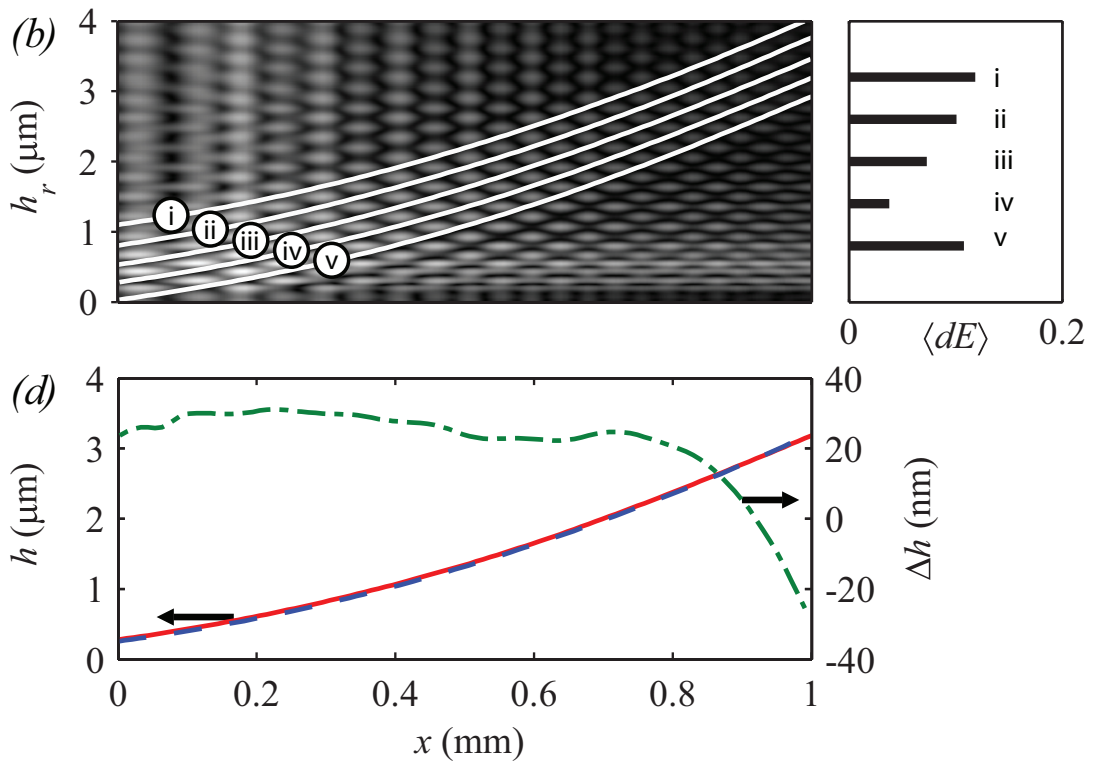

Figure 1.8: $(a)$ Color sample taken along a radial direction of a interference pattern. The origin $x=0$ is not where the lens and the glass slide are in contact. (b) Color difference with candidate profiles in white solid lines. $(c)$ Average color difference of candidate profiles shown in $(b)$. The selected profile is profile $(i v)$. (d) Comparison between the selected profile (shown in solid line) and the profile of the lens (shown in dashed line). The difference $\Delta h$ (shown in dashed-dotted line) between the selected profile and the lens's profile. 


\section{2}

\section{Maximal air bubble entrainment at liquid drop impact $^{*}$}

At impact of a liquid drop on a solid surface, an air bubble can be entrapped. Here, we show that two competing effects minimize the (relative) size of this entrained air bubble: for large drop impact velocity and large droplets the inertia of the liquid flattens the entrained bubble, whereas for small impact velocity and small droplets capillary forces minimize the entrained bubble. However, we demonstrate experimentally, theoretically, and numerically that in between there is an optimum, leading to maximal air bubble entrapment. For a $1.8 \mathrm{~mm}$ diameter ethanol droplet this optimum is achieved at an impact velocity of $0.25 \mathrm{~m} / \mathrm{s}$. Our results have a strong bearing on various applications in printing technology, microelectronics, immersion lithography, diagnostics, or agriculture.

${ }^{*}$ Published as: W. Bouwhuis, R. C. A. van der Veen, T. Tran, D. L. Keij, K. G. Winkels, I. R. Peters, D. van der Meer, C. Sun, J. H. Snoeijer and D. Lohse, Maximal air bubble entrainment at liquiddrop impact, Phys. Rev. Lett. 109, 264501 (2012). Experiments and analysis by RVDV, numerical simulations by WB, theory by DLK, KGW, IRP, DVDM and JHS, supervison by DVDM, CS, JHS, DL, discussion of the results by everyone. 


\subsection{Introduction}

The impact of liquid droplets on surfaces is omnipresent in nature and technology, ranging from falling raindrops to applications in agriculture and inkjet printing. The crucial question often is: how well does the liquid wet a surface? The traditional view is that it is the surface tension which gives a quantitative answer. However, it has been shown recently that an air bubble can be entrapped under a liquid drop as it impacts on the surface [17, 19, 22, 25, 26, 29]. Also Xu et al. [16, 30] revealed the important role of the surrounding air on the impact dynamics, including a possible splash formation. The mechanism works as follows [17, 19, 22, 29]: the air between the falling drop and the surface is strongly squeezed, leading to a pressure buildup in the air under the drop. The enhanced pressure results in a dimple formation in the droplet and eventually to the entrapment of an air bubble (Fig. 2.1a). The very simple question we ask and answer in this chapter is: for which impact velocity is the entrapped bubble maximal?

(a)

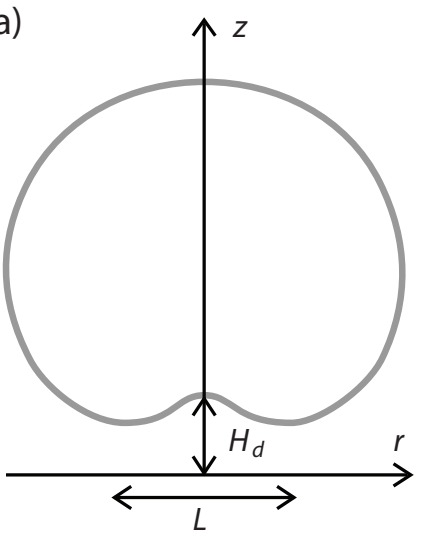

(b)

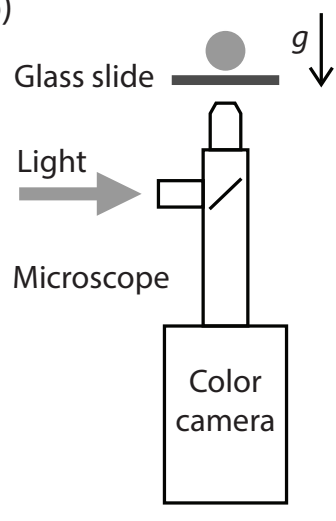

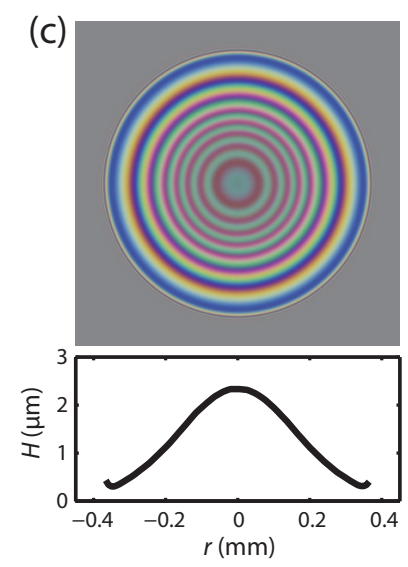

Figure 2.1: Experimental characterization of air bubble entrapment. (a) Sketch of dimple formation (not drawn to scale) just prior to impact. (b) Schematic of the experimental setup used to study droplet impact on smooth surfaces. An ethanol droplet of typical radius $R=0.9 \mathrm{~mm}$ falls on a glass slide of average roughness $10 \mathrm{~nm}$. The impact velocity is varied by varying the falling height of the droplet. For very small velocities below $0.31 \mathrm{~m} / \mathrm{s}$, the droplet is fixed at the tip of $0.4 \mathrm{~mm}$-diameter capillary that is vertically translated downwards at a constant velocity. The bottom view is captured by a high-speed color camera (SA2, Photron Inc.). The camera is connected to a long working-distance microscope and a $5 \times$ objective to obtain a $2 \mathrm{~mm}$ field of view. (c) An example of an interference pattern and the extracted air thickness profile. Note the difference in horizontal and vertical length scales. The exposure time was $1 / 15000 \mathrm{~s}$ and the typical frame rate of the recordings is 5000 frames per second. 


\subsection{Interferometry experiments}

Our experimental setup is shown in Fig. $2.1 \mathrm{~b}$ and is similar to that in chapter 1 and Ref. [31] where it is described in detail. An ethanol drop impacts on a smooth glass surface after detaching from a needle, or for velocities smaller than $0.32 \mathrm{~m} / \mathrm{s}$, after moving the needle downwards using a linear translation stage. A high-speed side view recording is used to measure the drop diameter and velocity. The experiment is carried out at room temperature. A synchronized bottom view recording by a high-speed color camera is used to measure the deformed shape of the liquid drop. Colored interference patterns are created by high-intensity coaxial white light, which reflects from both the glass surface and the bottom of the droplet. Using a color-matching approach in combination with known reference surfaces, the complete air thickness profile can be extracted (shown in Fig. 2.1c). For experiments done at larger impact velocities $(U>0.76 \mathrm{~m} / \mathrm{s})$, we use a pulse of diffused laser light triggered by an optical switch. The thickness of the air film at the rim is assumed to be zero, and the complete air thickness profile can then be obtained from the monochromatic fringe pattern. From these measurements we can determine the dimple height, $H_{d}$, and the volume of the entrained bubble, $V_{b}$, at the very moment of impact. This moment is defined by the first wetting of the surface. This is the moment when the concentric symmetry of the interference rings is lost, since due to unavoidable tiny tilts of the glass plate the wetting in general is non-axisymmetric. To calculate the bubble volume $V_{b}$, we integrate the thickness profile of the air layer trapped beneath the drop. Note that the dimple profiles and the volume of the entrained bubble are obtained before the wetting occurs, such that we do not have to take into account the properties of the surface (e.g. contact angle or roughness, which is of the order of $10 \mathrm{~nm}$ ). Alternatively, we can also measure the volume of the trapped bubble after impact when the liquid already wets the surface. Both measurements provide the same results. In the present chapter, we use the first approach.

The results are shown in Fig. 2.2. Clearly, both dimple height at impact and the size of the entrained bubble have a pronounced maximum as function of the impact velocity $U$. The corresponding impact velocity for which the air entrainment is maximal is in the regime $U_{0}=0.1-0.25 \mathrm{~m} / \mathrm{s}$ for an ethanol droplet of radius $R=0.9 \mathrm{~mm}$ (or the Stokes number $\mathrm{St}_{0}=0.3 \times 10^{4}-1 \times 10^{4}$ ). While length scales are given in multiples of the droplet radius $R$, following Brenner et al. [19, 22] we express the impact velocity $U$ in terms of the Stokes number St, defined with the dynamic air viscosity $\eta_{g}=1.82 \times 10^{-5} \mathrm{~Pa} \cdot \mathrm{s}$ and the liquid density $\rho_{l}=789 \mathrm{~kg} / \mathrm{m}^{3}$ as $\mathrm{St}=\rho_{l} R U / \eta_{g}=\rho_{l} / \rho_{g} \operatorname{Re}$, where $\mathrm{Re}=\rho_{g} R U / \eta_{g}$ is the standard Reynolds number of the gas. A further relevant parameter of the system is the surface tension $\gamma=$ $22 \mathrm{mN} / \mathrm{m}$, which can be expressed in terms of the Weber number We $=\rho_{l} R U^{2} / \gamma$ or in terms of the capillary number $\mathrm{Ca}=\eta_{g} U / \gamma=\mathrm{We} / \mathrm{St}$. 

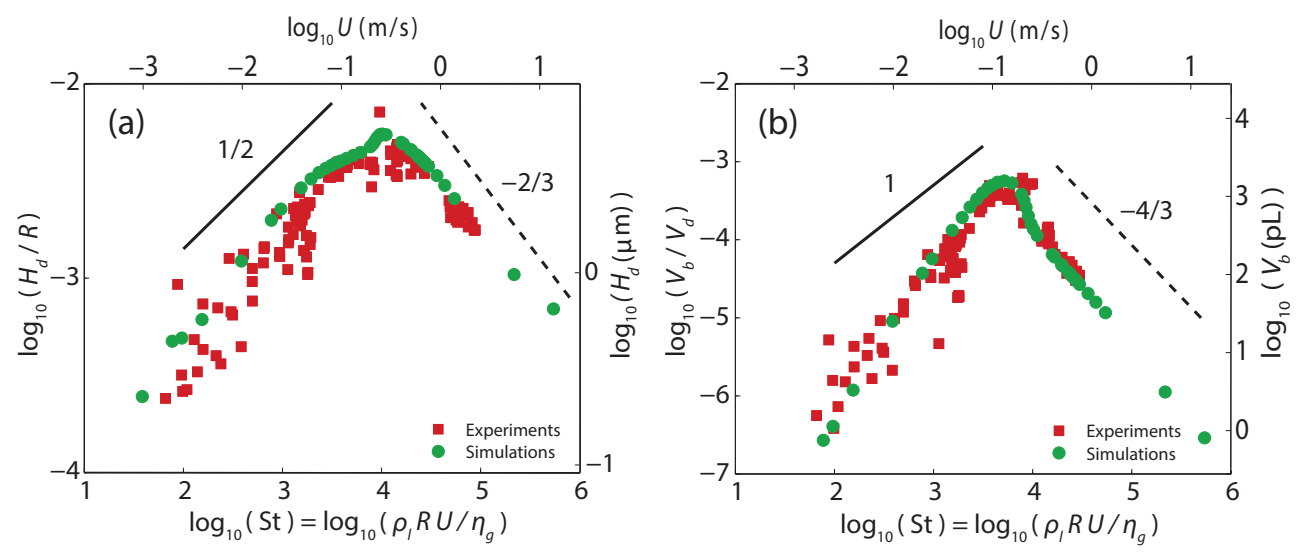

Figure 2.2: Maximum entrapment of air bubbles. (a) Dimple height $H_{d}$ and (b) entrained bubble volume $V_{b}$ as functions of the impact velocity $U$ (upper axes) and the Stokes number St (lower axes). The shape of the air layer can be characterized by the dimple height $H_{d}$ and the lateral extension $L$. Red squares correspond to high-speed color interferometry measurements, green dots correspond to numerical simulations. The straight lines correspond to the derived scaling laws in the capillary regime (solid) and inertial regime (dashed) with the respective scaling exponents.

\subsection{Boundary Integral simulations}

We compare and supplement our experimental findings on the dimple height at impact and the entrained bubble size to numerical results. The numerical simulation consists of an axisymmetric boundary integral (BI) simulation for the liquid droplet in which the droplet is assumed to obey potential flow, coupled to a lubrication approximation of the Stokes equation

$$
\frac{\partial P_{g}}{\partial r} \sim \eta_{g} \frac{\partial^{2} u_{r}}{\partial z^{2}}
$$

that describes the viscous, incompressible gas flow under the droplet [19-21, 32, 33]. Here, $z$ is the vertical direction, $P_{g}(r, t)$ is the gas pressure, while $u_{r}$ is the radially outward velocity in the gas parallel to the surface (Fig. 2.1a). Note that the gas flow under the droplet is indeed viscous: an upper bound for the Reynolds number relevant for the lubrication flow gives $U H_{d} / v_{g} \sim 0.1$ for the highest impact velocity, and is typically much smaller for most of our experiments.

We now give more details on the numerical simulation: the velocity field inside the droplet is described with a scalar velocity potential $\phi$, obeying the Laplace equation $\nabla^{2} \phi=0$. The axisymmetric droplet contour is described using cylindrical coordinates $r, z$ and is solved numerically by using the BI method; the simulations are based on the numerical code described by Refs. [34-36]. This BI simulation 
is an alternative way of solving the system of equations, compared to the method applied by Mani et al., 2010 [20], in which case a Hilbert transform method was applied. In contrast to Eggers et al., 2010 [33], we do not solve the complete NavierStokes equations, but do include dynamics of the air layer below the drop. The dynamic boundary condition valid on the droplet contours is given by the unsteady Bernoulli equation,

$$
\left(\frac{\partial \phi}{\partial t}+\frac{1}{2}|\nabla \phi|^{2}\right)=-g z-\frac{\gamma}{\rho_{l}} \kappa(r, t)-\frac{P_{g}(r, t)-P_{\infty}}{\rho_{l}} .
$$

Here $t$ is time, $g$ the acceleration of gravity, $z$ the absolute height, $\kappa(r, t)$ the interface curvature, and $P_{\infty}$ the far-field pressure. The key dynamical quantities in (2.2) are the gas pressure $P_{g}(r, t)$ and the interface curvature $\kappa(r, t)$. The curvature is related to the dimple profile $H(r, t)$ by the geometric relation

$$
\kappa(r, t)=\frac{\frac{\partial^{2} H(r, t)}{\partial r^{2}}}{\left(1+\left(\frac{\partial H(r, t)}{\partial r}\right)^{2}\right)^{3 / 2}}+\frac{\frac{\partial H(r, t)}{\partial r}}{r\left(1+\left(\frac{\partial H(r, t)}{\partial r}\right)^{2}\right)^{1 / 2}} .
$$

To close the problem, an additional equation is provided by the lubrication approximation for the viscous gas flow at the bottom of the droplet,

$$
\frac{\partial H(r, t)}{\partial t}-\frac{1}{r} \frac{\partial}{\partial r}\left[\frac{r(H(r, t))^{3}}{12 \eta_{g}} \frac{\partial P_{g}(r, t)}{\partial r}\right]=0,
$$

with boundary condition $\left.P_{g}\right|_{r=R}=P_{\infty}$; the gas pressure at the top of the droplet is set to atmospheric. Contrarily to Mani et al., 2010 [20], we do not incorporate effects of compressibility of the gas, since, following the analysis of Hicks et al., 2011 [32], there is little influence of compressibility in the regime that is studied here. The initial conditions for the simulations consist of a spherical droplet with radius $R$ having a downward velocity $U$. The initial height is taken sufficiently high for the pressure induced by the radial velocity profile to be still negligible as compared to the ambient pressure $(\sim 10 \mu \mathrm{m})$. The number of nodes on the droplet surface for which the BI equations are solved is of order 100, with node density increasing for $r \rightarrow 0$. The number of nodes and the size of the time steps vary during the simulation as a function of the local gap height and velocity of the droplet contour. The size of a time step is of order $10 \mathrm{~ns}$. For any number of nodes, the coupling between gap height and pressure profile breaks down for some small value of $H$, since the pressure diverges at vanishing thickness of the air layer. Consistent with the experimental resolution we continue our simulations until the minimum gap thickness reaches $0.4 \mu \mathrm{m}$, while ensuring that our algorithm remains accurate. This is the moment at which the values for $H_{d}$ and $V_{b}$ are extracted, which, as we will show below, have already achieved their final value much earlier. 

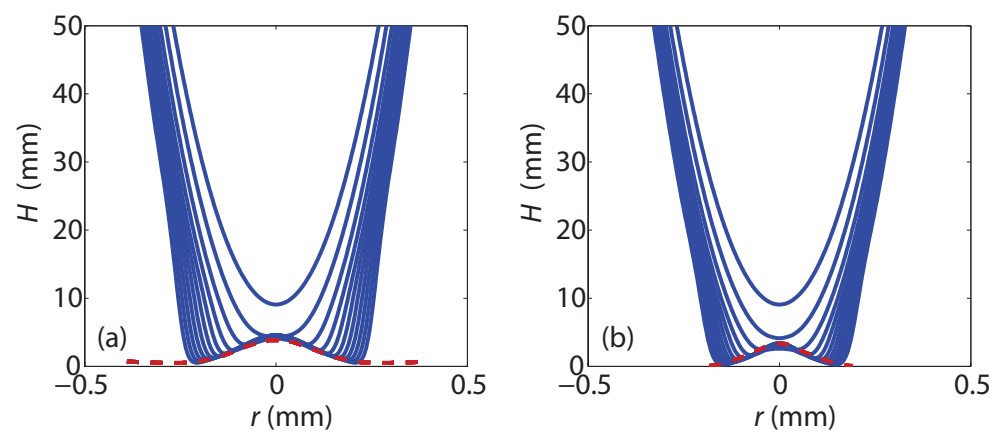

Figure 2.3: BI time evolution (solid blue lines) and an experimental profile (dashed red line) for ethanol droplet impact at (a): $U=0.32 \mathrm{~m} / \mathrm{s}\left(\mathrm{St}=1.25 \times 10^{4}\right)$ and $(\mathrm{b}): U=0.76 \mathrm{~m} / \mathrm{s}$ $\left(\mathrm{St}=2.97 \times 10^{4}\right)$.
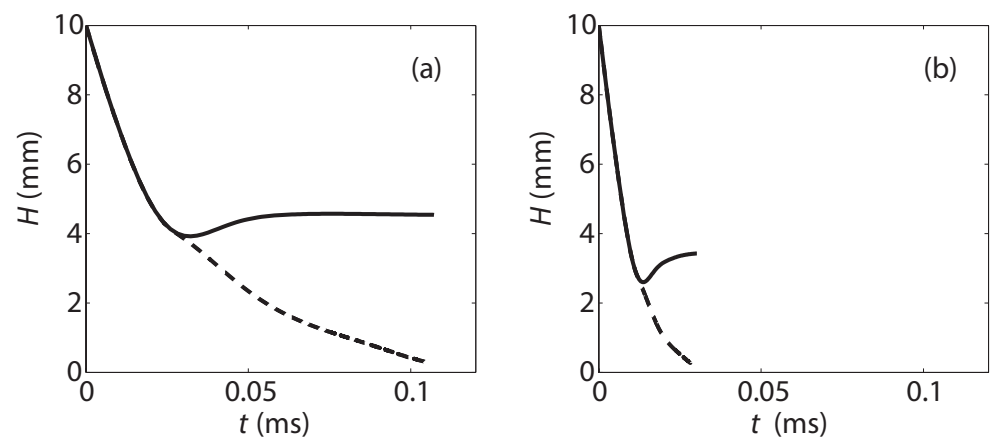

Figure 2.4: Time evolution of the height $H_{d}$ of the dimple (solid line) and the distance $d$ between the closest point of the liquid surface and the solid surface (dashed line) for the impact velocities (a): $U=0.32 \mathrm{~m} / \mathrm{s}$ and (b): $U=0.76 \mathrm{~m} / \mathrm{s}$. For both cases it is visible that the final dimple height is achieved way before the simulation is stopped at the cut-off $d=0.4 \mu \mathrm{m}$. 
Figure 2.3 shows the evolutions of the simulated ethanol droplets (blue lines). The two panels correspond to $U=0.32 \mathrm{~m} / \mathrm{s}$ and $U=0.76 \mathrm{~m} / \mathrm{s}$ (both at the right side of the maximum in Fig. 2.2) and are compared directly with the one-frame-results from experiment (red line). The comparison involves no adjustable parameters and reveals an excellent agreement for the dimple height. Given these satisfactory results, we can use the simulations to obtain further information of the time evolution of the air layer. Figure 2.4 shows the dimple height $H_{d}$ (solid line) and the minimum gap height $d$ (dashed line) as a function of time. Here, $t=0$ is defined by the moment at which the drop passes $H_{d}=10 \mu \mathrm{m}$. The separation of the solid and dashed lines marks the moment at which the center height, $H(r=0)$, no longer represents a minimum but has turned into a local maximum. This feature can also be inferred from the drop profiles shown in Fig. 2.3. The dimple height remains approximately constant at the later stages of Fig. 2.3. This implies that it is not critical to know the exact time at which the experimental profile is determined: the value of $H_{d}$ is not expected to vary much in this stage of the experiment.

The results of the numerical calculations of the dimple height and bubble volume are shown in Fig. 2.2, together with the experimental data, showing very good agreement: in particular, we observe pronounced maxima in the dimple size and in the entrained bubble volume. In the numerically obtained dimple height (and volume, to a lesser extent), we observe a jump exactly at the crossover regime. This jump originates from a change in the shape of the dimple. We focus on this in Fig. 2.5, which compares the experimental and numerical dimple profiles for an
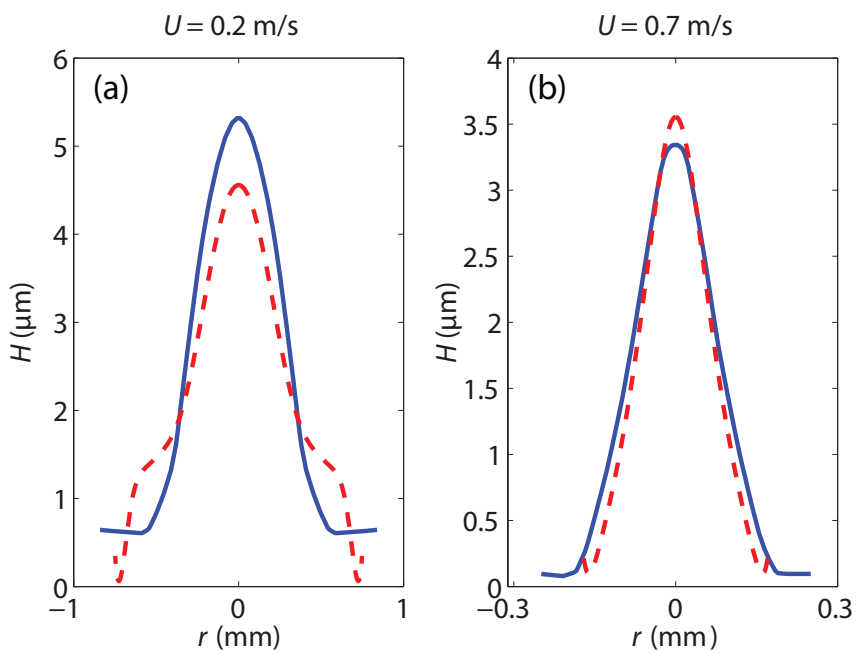

Figure 2.5: Comparison of experimental (blue) and numerical (red) dimple profiles for two different impact velocities; (a): $U=0.2 \mathrm{~m} / \mathrm{s}$ (St=7.8 $\times 10^{3}$; crossover regime) and (b): $U=0.7 \mathrm{~m} / \mathrm{s}\left(\mathrm{St}=2.7 \times 10^{4}\right.$; inertial regime). 
impact velocity at the crossover regime $(U=0.2 \mathrm{~m} / \mathrm{s})$ and an impact velocity in the inertial regime $(U=0.7 \mathrm{~m} / \mathrm{s})$ (these are parameters different from the ones chosen in Fig. 2.3 and Fig. 2.4). While the profiles are in excellent agreement within the St regime (both volume and dimple height), the numerical profile develops a "double dimple" at the crossover impact speed. Within the low St regime, we do not observe this double dimple, but the dimple is typically much broader. The variation in dimple shape results in the jump observed for the numerical dimple heights in the crossover regime (see Fig. 2.2a). In all cases, however, the dimple height $H_{d}$ and the entrapped bubble volume $V_{b}$ are in quantitative agreement without any adjustable parameters.

\subsection{Scaling laws}

Numerical and experimental results together suggest scaling laws $H_{d} / R \sim \mathrm{St}^{-2 / 3}$ for larger Stokes numbers, while $H_{d} / R \sim \mathrm{St}^{1 / 2}$ for smaller Stokes numbers. We will now theoretically derive these scaling laws. For large St we follow and extend Refs. [21, 22, 37]: the horizontal length scale $L$ of the dimple extension (see Fig. 2.1a) follows from geometrical arguments as $L \sim \sqrt{H_{d} R}$, and $u_{r}$ from mass conservation as $u_{r} \sim U L / H_{d}$. The Stokes equation (2.1) suggests $P_{g} \sim L \eta_{g} u_{r} / H_{d}^{2}$ as estimate for the gas pressure below the falling drop at touch-down. The liquid pressure $P_{l}$ can be estimated from the unsteady Bernoulli equation: dimensional analysis gives the deceleration timescale $H_{d} / U$ and the potential in the liquid $\sim U L$, resulting in $P_{l} \sim \rho_{l} U^{2} L / H_{d}$. Since the liquid drop will be deformed when $P_{g} \sim P_{l}$, one finally obtains the scaling for the dimple height and the bubble volume:

$$
H_{d} \sim R \mathrm{St}^{-2 / 3}, \quad V_{b} \sim L^{2} H_{d} \sim R^{3} \mathrm{St}^{-4 / 3} .
$$

This describes the air bubble in the inertial regime, i.e. large impact velocities, in agreement with our experimental and numerical findings.

For small St, corresponding to small impact velocity and small droplet radius, capillarity will take over and try to smoothen the dimple out. Then the pressure inside the gas must be balanced with the Laplace pressure $\gamma \kappa$ that is imposed at the liquid-air interface, where $\kappa \sim H_{d} / L^{2}$ is the curvature of the dimple. Using once more that the gas pressure $P_{g} \sim L \eta_{g} u_{r} / H_{d}^{2}$, one obtains

$$
\begin{aligned}
\frac{H_{d}}{R} & \sim \sqrt{\mathrm{Ca}} \sim \sqrt{\mathrm{We} / \mathrm{St}} \sim \frac{\eta_{g}}{\sqrt{\gamma \rho_{l} R}} \mathrm{St}^{1 / 2}, \\
\frac{V_{b}}{R^{3}} & \sim \frac{\eta_{g}^{2}}{\gamma \rho_{l} R} \mathrm{St},
\end{aligned}
$$

as scaling in the capillary regime. Again, this is consistent with the experimental and numerical findings. The crossover between the regimes, corresponding to the 
maximal air bubble entrainment, occurs at

$$
\mathrm{St}_{o} \sim \mathrm{Ca}_{o}^{-3 / 4} \quad \text { or } \quad U_{o} \sim \frac{\eta_{g}^{1 / 7} \gamma^{3 / 7}}{\rho_{l}^{4 / 7} R^{4 / 7}}
$$

Using prefactors obtained from our experimental data in Fig. 2.2, for an ethanol droplet of $0.9 \mathrm{~mm}$ radius, this translates to an impact velocity $U_{o}=0.25 \mathrm{~m} / \mathrm{s}$.

\subsection{Conclusion}

What is the physical reason for the maximum? For higher velocities inertia dominates and flattens the droplet at impact. For lower velocities and/or smaller droplets the capillary forces try to keep the drop spherical. In between these two regimes the maximal air entrainment under the droplet is achieved.

For many applications air entrainment is undesirable and maximal wetting must be achieved. This holds for immersion lithography, wafer drying, glueing, agricultural applications, etcetera [38, 39]. Intriguingly, for inkjet drops of radius $R \sim 10 \mu \mathrm{m}$, the optimal velocity according to (2.7) is approximately $1 \mathrm{~m} / \mathrm{s}$. This lies exactly in the range at which inkjet usually operates (typically a few $\mathrm{m} / \mathrm{s}$ ), and relatively large bubbles will thus be entrapped [25]. For immersion lithography the entrapment of even micron-sized bubbles can cause practical limitations [38, 39]. This technology is based on optical imaging of nanoscale structures, for which the optics is immersed in water to push the limits of spatial resolution. Clearly, it is crucial to avoid bubbles or to minimize their size, which also has bearing in cleaning and drying of wafers. Ideally, one should stay as far as possible from the optimal air entrainment impact velocity. Our findings will help to achieve this goal and thus optimal wetting. 


\section{3}

\section{How microstructures affect air film dynamics prior to drop impact*}

When a drop impacts on a surface, a dimple can be formed due to the increased air pressure beneath the drop before it wets the surface. We employ the high-speed color interferometry technique developed in chapter 1 to measure the evolution of the air layer profiles under millimeter-sized drops impacting on hydrophobic micropatterned surfaces for impact velocities of typically $0.4 \mathrm{~m} / \mathrm{s}$. We account for the impact phenomena and show the influence of the micropillar spacing and height on the air layer profiles. A decrease in pillar spacing increases the height of the air dimple below the impacting drop. Before complete wetting, when the impacting drop only wets the top of the pillars, the air-droplet interface deforms in between the pillars. For large pillar heights the deformation is larger, but the dimple height is hardly influenced.

${ }^{*}$ Published as: Roeland C. A. van der Veen, Maurice H. W. Hendrix, Tuan Tran, Chao Sun, Peichun Amy Tsai and Detlef Lohse, How microstructures affect air film dynamics prior to drop impact, Soft Matter 10, 3703-3707 (2014). Measurements done by RVDV and MHWH, substrates supplied by PAT, writing by RVDV, supervision by CS, TT, PAT and DL, discussion of the results and proofreading of the manuscript by everyone. 


\subsection{Introduction}

A drop impacting on a solid surface causes the air pressure underneath the drop to increase due to the thin air layer that needs to be squeezed out before the drop wets the surface. This build-up of air pressure can deform the drop, causing a non-equilibrium dimple, which may result in air bubble entrainment $[19,20,25$, 40]. The role of air is also important in the macroscopic splashing behaviour of drops impacting on smooth surfaces [17, 19, 20, 26, 29, 30] or micropatterned surfaces $[16,41,42]$. In the latter case, the interplay of the trapped air and the geometry of the structure determines the complex outcome of a drop impact event, e.g. directional splashing [43]. Previously, the evolution of the air-liquid interface of a gently deposited drop on a micropatterned surface, with essentially zero impact velocity, has been investigated, focusing on the Cassie-Baxter to Wenzel transition [44-48]. However, when a drop approaches a microstructure with a nonzero impact speed, the dynamics of the interface will be very different due to the increase of pressure in the microscopic air layer at the bottom of the drop before wetting. The evolution of the air film interacting with the microstructure during such an impact event has not been quantified yet. In this chapter we focus on the trapped air layer between an impacting water drop and various superhydrophobic micropatterned surfaces. We quantitatively measure the evolution of the air film thickness during drop impact, using the color interferometry method which has been used in chapters 1 and 2 to infer the dynamics of the air film under a drop impacting onto a smooth surface. The influence of the micropillar arrangement and size on the air-liquid interface is not only important from a fundamental point of view, but is also relevant for many industrial and technical applications, in which the size of entrained air bubbles needs to be controlled. Examples are ink-jet printing [25], spray coating and spray cooling. In the case of spray cooling the Leidenfrost phenomenon is crucial [49-52].

\subsection{Experiments}

To study the impact dynamics, we employ the setup shown in Fig. 3.1(a). This setup is similar to that in chapters 1 and 2, with the exception of the use of hydrophobic micropatterned surfaces instead of smooth ones. A milli-Q water drop detaches from a needle after growing quasi-statically and impacts on the surface. The solid substrate consists of glass micropillars regularly arranged in a square lattice, with pillar width $W$, pillar spacing $S$ and pillar height $H$, as shown in Fig. 3.1(b) and (c). The substrate has a hydrophobic fluorocarbon (FC) layer ( $\approx 100 \mathrm{~nm}$ thick) [53], which gives a static contact angle of $106 \pm 2^{\circ}$ for water on smooth glass. Micropatterned surfaces with hydrophilic coatings were also tried, 
(a)

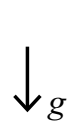

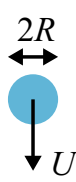

Micropatterned glass slide

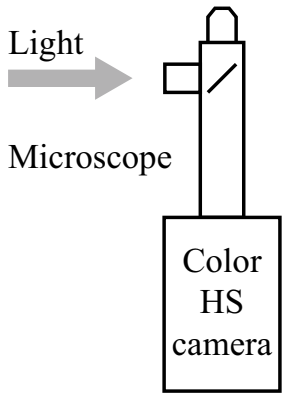

(b)

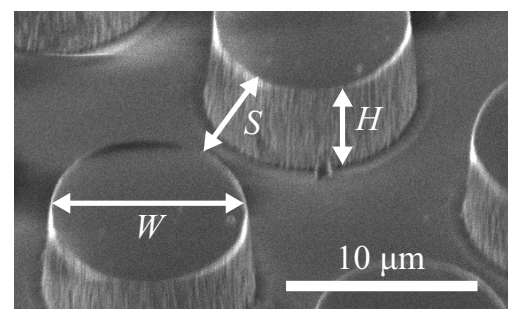

(c)

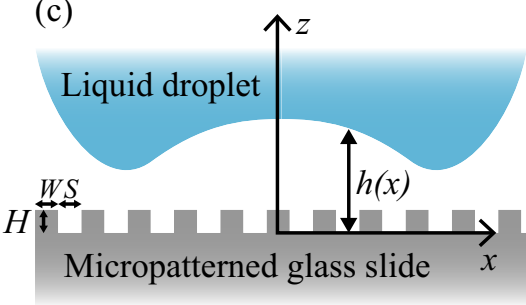

Figure 3.1: Experimental characterization of the drop impact experiments. (a) Schematic of the experimental setup used to study drop impact using high-speed color inferometry. A water drop with a typical radius of $R=1 \mathrm{~mm}$ falls on a transparent hydrophobic micropatterned or hydrophilic smooth glass slide. (b) Scanning electron microscope (SEM) image of a representative micropatterned surface, showing the width $(W)$, spacing $(S)$ and height $(H)$ of the micropillars. (c) Sketch of the dimple formation (not drawn to scale) prior to impact. The height $h(x)$ of the air film is defined to be from the bottom of the pillars to the bottom of the liquid drop.

but found to be less suitable to study air entrapment, because the liquid touches the pillars early in the impact process and consequently quickly completely wets the surface (Wenzel state $[46,54]$ ). In this work we only used hydrophobic coated micropatterned surfaces and compare with the case of smooth glass slides (Menzel microscope slides, average roughness $\approx 10 \mathrm{~nm}$ ). The impact velocity, which is typically $U=0.4 \mathrm{~m} / \mathrm{s}$ in the present work, can be varied by changing the falling height of the drop. The drop radius and velocity is measured using a high-speed side-view camera (SA1, Photron Inc.). The bottom view is captured by a synchronized high-speed color camera (SA2, Photron Inc.) operating at 10000 or 20000 frames per second (fps). The camera is connected to a long-working-distance microscope (Navitar Inc.) and a 10x objective with a $1 \mathrm{~mm}$ field of view to measure the air-water interface, or equivalently, the shape of the air film between the drop and the surface. Broad-spectrum white light from a high-intensity mercury vapor lamp (Olympus ILP-1) is fed into the coaxial light port of the long-workingdistance microscope. This light reflects from both the top surface of the substrate and the bottom surface of the drop, creating colored interference patterns. These 
colored patterns can be used to obtain the absolute thickness of the film in question. A color-matching approach in combination with known reference surfaces is employed, see also the appendix 3.A.

\subsection{Results and discussion}

A representative 2-D and 3-D reconstruction of the air layer resulting from impact on a substrate with pillar width $W=100 \mu \mathrm{m}$ and spacing $S=100 \mu \mathrm{m}$ is shown in Fig. 3.2(a) and (b). As is the case with smooth surfaces, upon drop impact with a structured surface, the liquid at the bottom is deformed and a dimple is created due to a pressure build-up in the air layer. In Fig. 3.2(b) it can be seen that the pressure build-up also happens very locally; above the pillar at $(x, y)=$ $(0.3 \mathrm{~mm}, 0.05 \mathrm{~mm})$ a local maxima in the air layer thickness is formed. The liquid first only wets the top of the pillars (see Fig. 3.2(a), Cassie-Baxter or Fakir state [5557]), trapping air in between the pillars. The small fringe spacing close to the pillars, corresponding to a steep profile, suggests that the liquid is pinned at the top pillar edges. As a further indication, a profile between two adjacent pillars is constructed (Fig. 3.2(c)). The color interference technique allows us to resolve a large part of the profile in between the pillars. This is important in many aspects, such as for understanding the effect of surface structure on the dynamic Leidenfrost temperature [52], which is affected by the additional surface area due to the protruding air-water interface. Another example is the application of heterogeneous porous catalysts [58], where it is desirable to quantify the exact contact area. Profiles at two instants in time both show, when extrapolating, that the liquid surface is connected very close to the top of the pillars. Considering the measured profiles and the fact that the Fakir state is (meta-)stable [45], we conclude that the liquid is pinned at the edge of the top of the pillars at least up to $2.3 \mathrm{~ms}$ in the current experiment. Having treated a single snapshot, we now turn our attention to the dynamic evolution of the air film during impact. In the case of smaller pillar width $W=10 \mu \mathrm{m}$ and spacing $S=20 \mu \mathrm{m}$, the air layer shapes and impact dynamics are reminiscent of impact on a smooth surface, see Fig. 3.3. A cross-section of the air layer is made through the space between two rows of pillars. Two distinct areas can be discerned. One is the central part of the dimple, of which the symmetry is not influenced much by the presence of the pillars. The second one is the outer region where the top of the pillars are wetted and the liquid bends down into the gaps to a height of approximately $0.3 \mu \mathrm{m}$, less than half of the pillar height. At an unpredictable time the liquid unpins (outside of the frame) from the pillars and starts to completely wet the surface. As can be the case with smooth surfaces, the dimple evolves into an entrapped air bubble. The exact time at which wetting starts varies between experiments, because it strongly depends on small irregularities or con- 
(a)
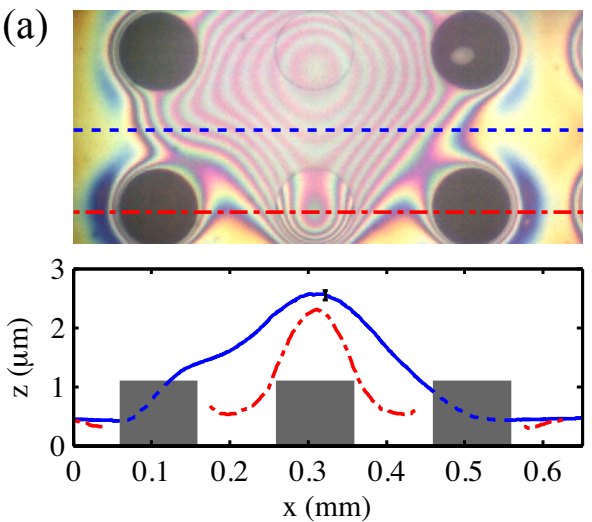

(c)
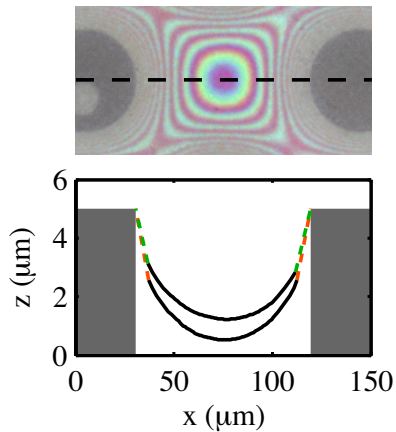

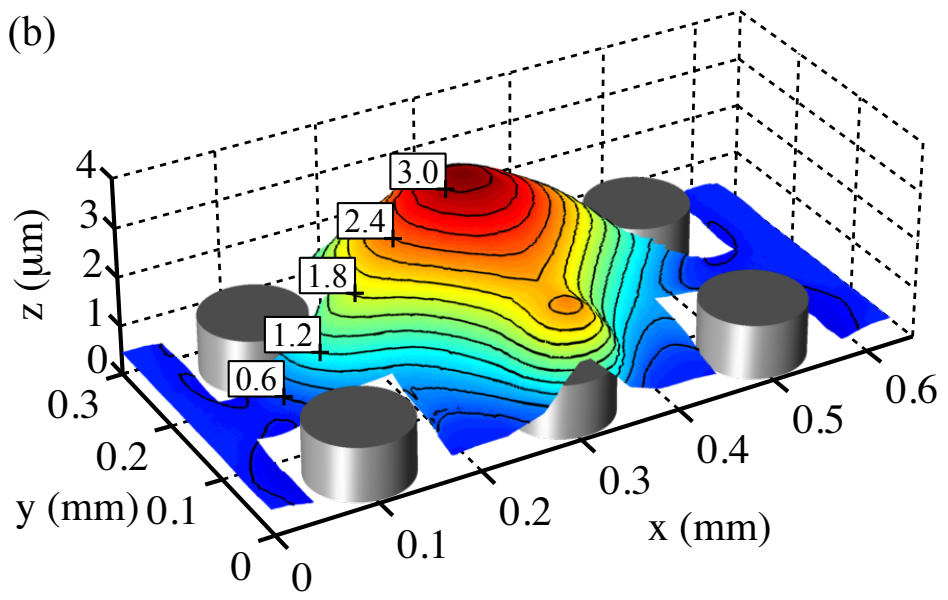

Figure 3.2: Two-dimensional (2-D) and three-dimensional (3-D) reconstruction of the air layer profile between an impacting drop and a micropatterned surface. (a) Top: Snapshot of the interference pattern created by light interference between the surface and the bottom of the drop. The liquid wets the top of four of the six pillars present in this picture. The drop radius is $R=1 \mathrm{~mm}$, the impact velocity is $U=0.4 \mathrm{~m} / \mathrm{s}$, the microstructure properties are $W=100 \mu \mathrm{m}, S=100 \mu \mathrm{m}, H=1.1 \mu \mathrm{m}$. Bottom: Air layer profiles along the two lines shown at the top. Note the difference in horizontal and vertical scale. (b) 3-D reconstruction of the air layer. Black iso-height lines are shown with labels in $\mu \mathrm{m}$. The dimple is deformed by the presence of the micropillars. (c) Top: Interference pattern between two pillars at $t=2.3 \pm 0.1 \mathrm{~ms}(U=0.3 \mathrm{~m} / \mathrm{s}, R=1 \mathrm{~mm}, W=50 \mu \mathrm{m}, S=50 \mu \mathrm{m}, H=5.1 \mu \mathrm{m})$. See the appendix 3.A for a definition of the reference time. Bottom: Air layer profiles for $t=0.3 \mathrm{~ms}$ (upper curve) and $t=2.3 \mathrm{~ms}$ (lower curve). The solid lines represent the profiles that are constructed using the color interference technique described in the text, while the dashed lines are a linear interpolation to the top edge of the pillars, serving as a guide to the eye. The shape and dynamics of this pattern suggest pinning of the liquid to the top edge of the pillars. 


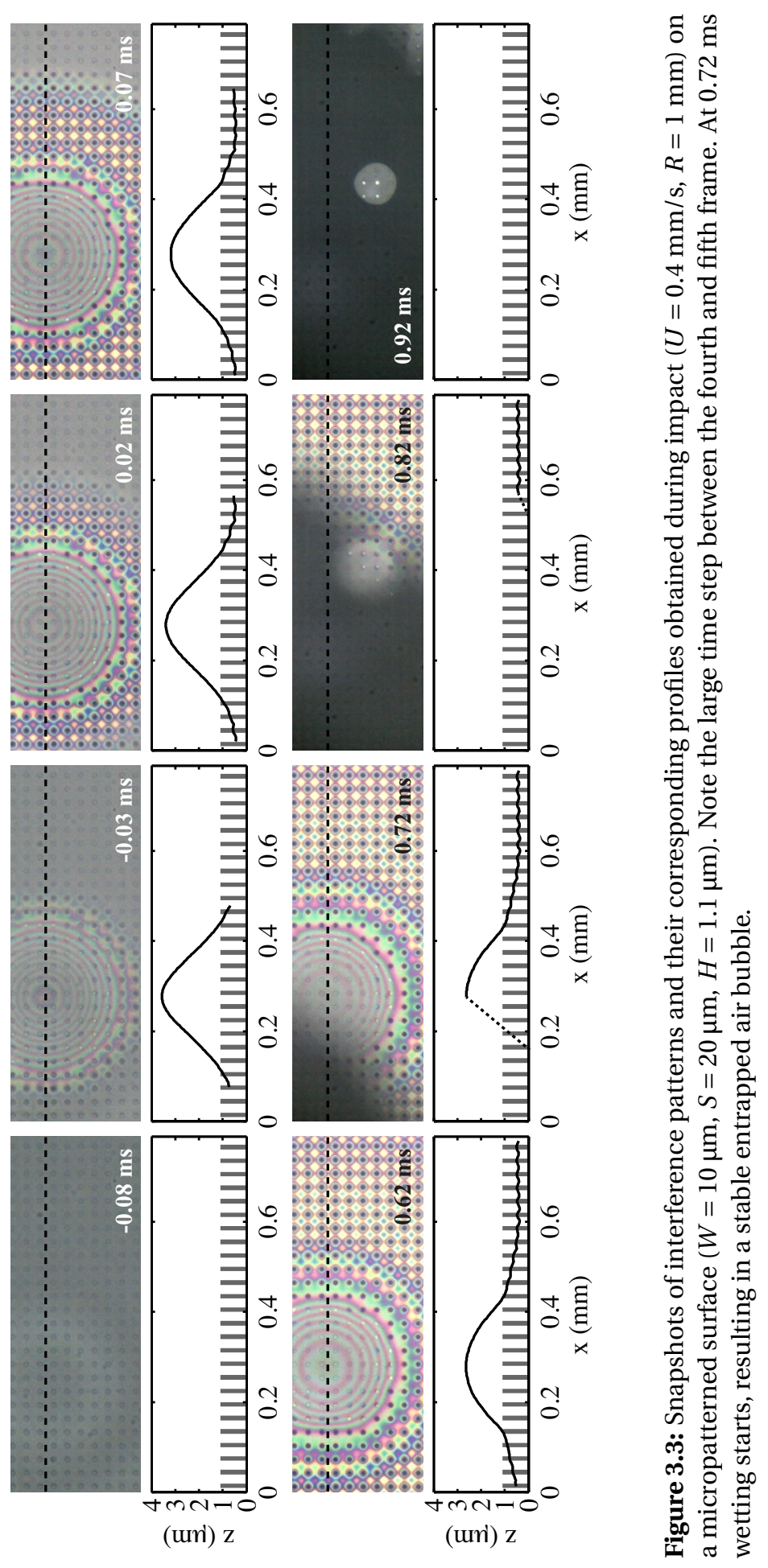


taminants. Together with unavoidable tiny tilts or non-symmetrical releases of the drops, these effects can also cause the non-axisymmetric wetting behavior.

To study the effect of the micropillar size and arrangement on the impact dynamics, we measure air layer profiles beneath the drops from the moment fringes are first visible until the moment wetting starts. From the measurements the height of the air film at the center of the dimple is extracted. The micropillar width and spacing are varied, while the pillar height and impact velocity are kept constant. In Fig. 3.4(a) it can be seen that the central part of the dimple is quite symmetrical for all pillar arrangements considered. By inspecting the interference fringes one can note that the dimple height is different for every arrangement. This is quantified in Fig. 3.4(b) by plotting the maximum dimple height $H_{d}$ versus time for seven different micropillar arrangements and one smooth surface case. Time $t=0$ is defined as the moment where fringes are first visible in the frame. This depends on imaging variables such as depth of field, but these were kept constant between experiments (see appendix 3.A for an explanation on the sub-frame accuracy). The first thing to notice when comparing the maximum dimple height evolutions is that all have very similar shape and seem to be shifted vertically with respect to each other. This is quantified in Fig. 3.4(c), where the difference of all profiles as compared to the smooth surface case is shown. In an interval of 0.2 ms the fluctuations of these lines are within the measurement error of $100 \mathrm{~nm}$. The presence of protruding pillars in the air layer creates an additional pressure build-up, or resistance, to the impacting drop, increasing the height of the dimple. For equal pillar width and smaller pillar spacing, the dimple is pushed up higher because air can escape less easily. Outside of the dimple, the liquid wets the top of the pillars, so there are only channels with cross-sectional area of less than $S \cdot H$ through which air can escape. Compressibility effects are not expected to play a role at these low impact velocities [32]. When comparing only cases with equal ratio of pillar spacing to pillar width $S / W$ (e.g. red crosses and light blue squares in Fig. 3.4(b)), the total area through which air can escape is constant. Nonetheless, the structures with larger dimensions have a smaller dimple height, suggesting that air escapes more easily in those cases. This can be qualitatively explained using aerodynamical resistance of the structure to the air flow, which is larger for many small channels than for a smaller number of large channels with equal total cross-sectional area. The geometrical parameter $W /(W+S)$ is used to show the dependence of the dimple height on the micropillar arrangement (Fig. 3.4(d)). The factor $W /(W+S)$ is a measure for the relative pillar width, and corresponds to smooth surfaces at values of 0 and 1 . The four different values in between show a monotonic increase of the normalized dimple height with the relative pillar width $W /(W+S)$. It will be of interest to theoretically explain this dependence, which is beyond the scope of the present work. 
(a)
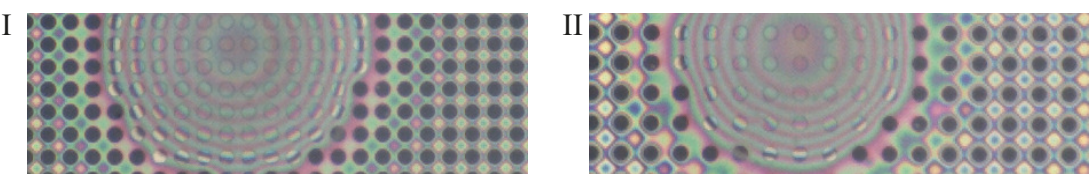

III
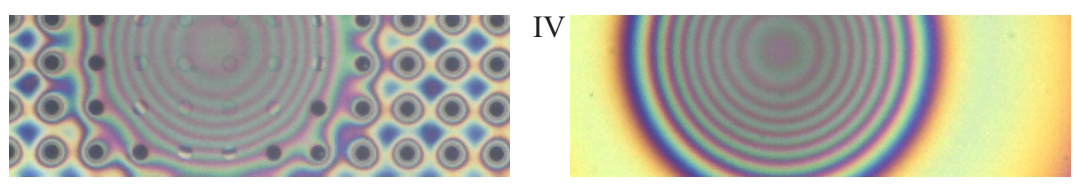

(b)
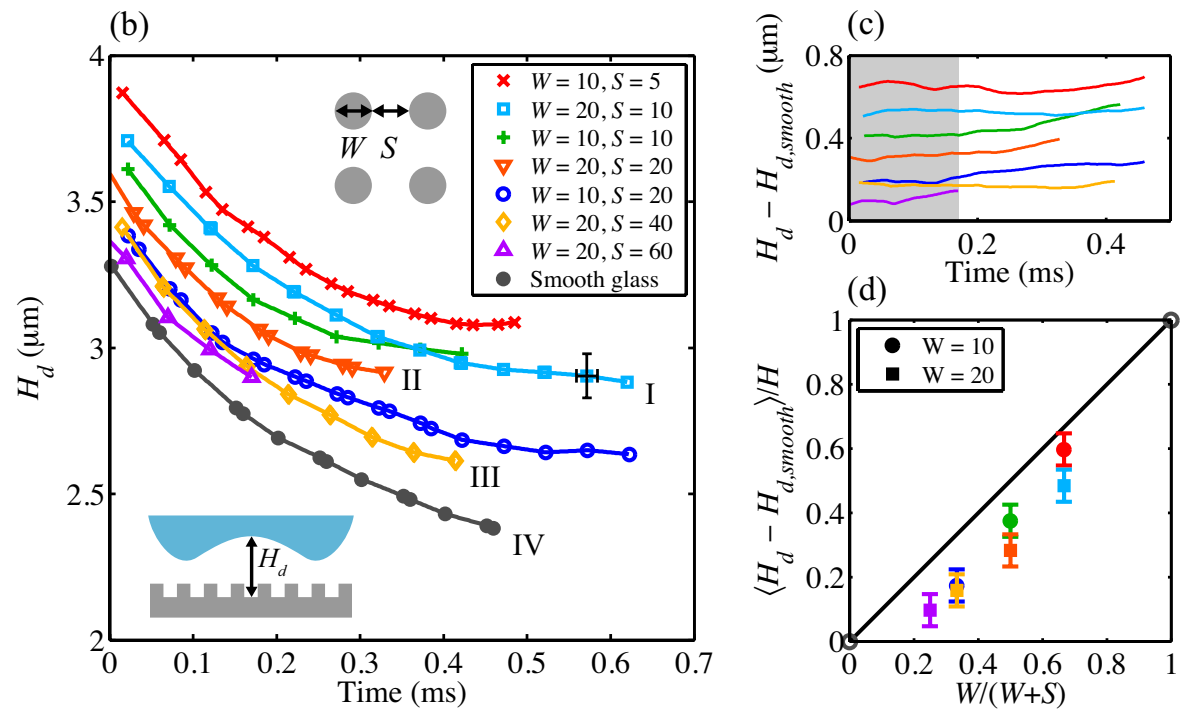

Figure 3.4: Overview of microstructure dependence for fixed pillar height $H=1.1 \mu \mathrm{m}$ ( $U=0.4 \mathrm{~m} / \mathrm{s}, R=1 \mathrm{~mm}$ ). (a) Four snapshots at $t=0.23 \pm 0.03 \mathrm{~ms}$ of impact on micropatterned surfaces $(W=20 \mu \mathrm{m})$ with spacings $S=10,20,40 \mu \mathrm{m}$, and on a smooth glass slide. (b) Evolution of maximum dimple height $H_{d}$ with time. Every line consists of an average of two individual experiments, which are independently shifted in time according to the method described in the appendix 3.A. The variation between every experiment falls within the systematic error of $150 \mathrm{~nm}$ which we attribute to the method of analysis. (c) The difference between the six microstructure experiments and the smooth surface case, showing that the shape of $H_{d}(t)$ hardly depends on the type of microstructure. The grey area shows the interval $0 \mathrm{~ms}<t<0.17 \mathrm{~ms}$ used to determine the average dimple height difference. (d) Average dimple height difference normalized by the pillar height $H=1.1 \mu \mathrm{m}$ versus the pillar width parameter $W /(W+S)$. A value of zero corresponds to a smooth surface at $z=0$, a value of one corresponds to a smooth surface at $z=H$. The wider the pillars compared to the unit cell, the higher the air dimple. In addition, all cases with a smaller pillar width ( $W=10 \mu \mathrm{m}$ compared to $W=20 \mu \mathrm{m}$ ) have a larger dimple height for equal parameter $W /(W+S)$. 
(a)

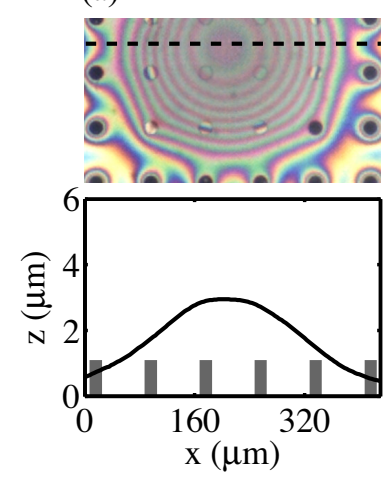

(b)

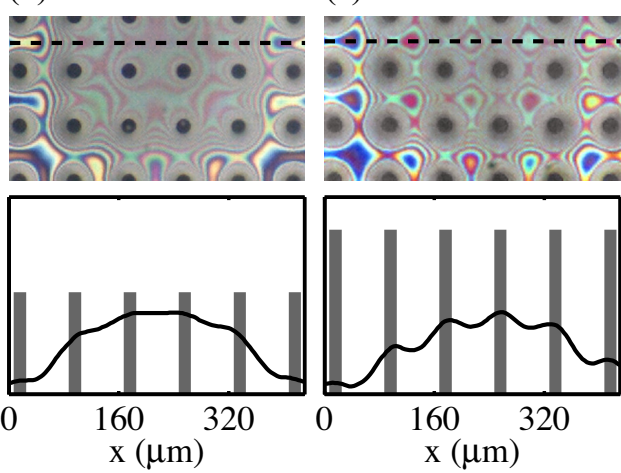

Figure 3.5: Influence of pillar height. (a), (b) and (c) Snapshots of measurements at $t=0.15 \pm 0.03 \mathrm{~ms}$ with $W=20 \mu \mathrm{m}$ and $S=60 \mu \mathrm{m}$, but different pillar heights of $H=$ $1.1,3.1$, and $5 \mu \mathrm{m}$ respectively $(U=0.4 \mathrm{~m} / \mathrm{s}, R=1 \mathrm{~mm})$. The profile is evaluated along a line between the pillars, showing that especially for large pillar height, the air film significantly penetrates the microstructure. The dimple height does not strongly depend on the pillar height.

Besides the pillar width and spacing, the influence of the pillar height is also of interest. Fig. 3.5 shows snapshots of the interference patterns and corresponding dimple shapes for three different pillar heights, keeping the pillar width and spacing constant. For a pillar height $H=1.1 \mu \mathrm{m}$, which is smaller than the typical dimple height, the dimple itself is not disturbed and still symmetric. In the case of a pillar height of $H=3.1 \mu \mathrm{m}$, the dimple retains its general shape, but the liquid wets the top of all the pillars and the dimple is much more deformed. The same characteristics can be seen for $H=5.1 \mu \mathrm{m}$, but the dimple is deformed even more. As described before and shown in Fig. 3.1(c), the liquid is still connected to the top of the pillars. When comparing these three cases, it can be seen that the pillar height, for a relatively small value of the relative pillar width $W /(W+S)=0.25$, does not significantly influence the dimple height. 


\subsection{Conclusion}

Using a high-speed color interferometry technique, the evolution of the air layer morphology between impacting drops and hydrophobic micropatterned surfaces was experimentally quantified. The liquid-air interface globally resembles the azimuthally symmetric dimple on smooth surfaces, however with local symmetrybreaking deformation caused by the microstructure. We found that the central dimple height is increased due to presence of protruding pillars, which create an additional pressure build-up. Finally, the maximum dimple height is hardly influenced by the height of the pillars. These results and the employed imaging technique can facilitate the understanding of drop impact on superhydrophobic surfaces.

\section{A Details on the method}

\section{Method}

A color-matching approach in combination with known reference surfaces is used to construct air layer profiles, see chapter 1 . For this method it is beneficial to obtain a good match between the white balance and exposure of the experiments and reference color database. To this end, the white balance and image contrast are independently optimized to obtain the smallest average color difference. After finding an approximate profile, this is optimized by vertically translating every data point (with steps of $3 \mu \mathrm{m}$ in the horizontal direction). For every point the minimal color difference is found, resulting in the final air layer profile, which is then smoothed with a moving average filter of width $4.5 \mu \mathrm{m}$.

Once the final air film profile is obtained, we can extract information such as the dimple volume and dimple height from this data. The dimple height is found by fitting the dimple profile with a second order polynomial to a width of $0.1 \mathrm{~mm}$ around the center.

\section{Error analysis}

The inaccuracies in determining the air layer profiles arise from several sources. One is in the comparison with a reference color database which has an intrinsic error of approximately $40 \mathrm{~nm}$ (see chapter 1). This is a systematic error which is equal for all experiments and therefore not relevant in for example Fig.4(b) where height differences between different experiments are considered. Another source of inaccuracies is in the determination of the line with smallest color difference. This results from white balance and exposure difference between measurements and reference images, and is slightly higher for larger air thicknesses because there 
the contrast is smaller. For an air layer thickness below $1 \mu \mathrm{m}$ this error is $50 \mathrm{~nm}$ and beyond $1 \mu \mathrm{m}$ it is approximately $100 \mathrm{~nm}$.

\section{Determination of $t=0$}

The reference time $t=0$ is determined by the time at which fringes are first fully visible in the image. This depends on the depth of field and/or coherence length of the imaging setup, but in the present experiments these variables are constant. In the case of a recording frame rate of 20000 frames per second (fps) and impact velocities of the order of $0.4 \mathrm{~m} / \mathrm{s}$, one typically first sees some faint fringes, after which in the next frames the fringes are fully visible.

To achieve sub-frame accuracy of the reference time $t=0$, we use the following method. Consider frame 0 without a visible fringe pattern, frame 1 with fringes which are visible to a certain degree, and frame 2 with completely visible fringes. The measure for visibility of fringes we use is the standard deviation of the intensity fluctuation along a line across the dimple, averaged over all three color channels. If the fringes in frame 1 are almost completely visible, we set $t=0$ to be at the beginning of frame 1 . If they are very faint, $t=0$ is set close to the beginning of frame 2. The start time from frame 1 is then $\Delta t=1 / \mathrm{fps} \cdot\left(1-\left(\sigma_{2}-\sigma_{1}\right) /\left(\sigma_{3}-\sigma_{1}\right)\right)$. Standard deviations are used to get a measure of the intensity fluctuation of the fringes themselves, and exclude variations in overall exposure. The accuracy we can achieve in $\Delta t$. fps is about 0.5 , meaning half a frame. This method can be expanded to higher frame rates or lower impact velocities by including more than three frames. 


\section{Part II}

\section{Taylor-Couette turbulence}





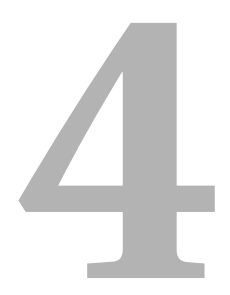

\section{The Boiling Twente Taylor-Couette (BTTC) facility: temperature controlled turbulent flow between in- dependently rotating, coaxial cylinders*}

A new Taylor-Couette system has been designed and constructed with precise temperature control. Two concentric independently rotating cylinders are able to rotate at maximum rates of $f_{i}= \pm 20 \mathrm{~Hz}$ for the inner cylinder and $f_{o}= \pm 10 \mathrm{~Hz}$ for the outer cylinder. The inner cylinder has an outside radius of $r_{i}=75 \mathrm{~mm}$, and the outer cylinder has an inside radius of $r_{o}=105 \mathrm{~mm}$, resulting in a gap of $d=30 \mathrm{~mm}$. The height of the gap is $L=549 \mathrm{~mm}$, giving a volume of $V=9.3 \mathrm{~L}$. The geometric parameters are $\eta=r_{i} / r_{o}=0.714$ and $\Gamma=L / d=18.3$. With water as working fluid at room temperature the Reynolds numbers that can be achieved are $R_{i}=\omega_{i} r_{i}\left(r_{o}-r_{i}\right) / v=$ $2.8 \times 10^{5}$ and $\operatorname{Re}_{o}=\omega_{o} r_{o}\left(r_{o}-r_{i}\right) / v=2 \times 10^{5}$, or a combined Reynolds number of up to $R e=\left(\omega_{i} r_{i}-\omega_{o} r_{o}\right)\left(r_{o}-r_{i}\right) / v=4.8 \times 10^{5}$. If the working fluid is changed to the fluorinated liquid FC-3284 with kinematic viscosity $0.42 \mathrm{cSt}$, the combined Reynolds number can reach $R e=1.1 \times 10^{6}$. The apparatus features precise temperature control of the outer and inner cylinder separately, and is fully optically accessible from the side and top. The new facility offers the possibility to accurately study the process of boiling inside a turbulent flow and its effect on the flow.

${ }^{*}$ Published as: Sander G. Huisman, Roeland C. A. van der Veen, Gert-Wim H. Bruggert, Detlef Lohse and Chao Sun, The Boiling Twente Taylor-Couette (BTTC) facility: temperature controlled turbulent flow between independently rotating, coaxial cylinders, Rev. Sci. Inst. 6, 065108 (2015). Setup design by $\mathrm{SH}$ and GWHB, measurements by $\mathrm{SH}$ and RVDV, writing by SH, supervision by CS and DL, discussion of the results and proofreading of the manuscript by SH, RVDV, CS and DL. 


\subsection{Introduction}

Though well known in the context of rheology, the Taylor-Couette geometry-a geometry in which fluid is bound between two concentric rotating cylindershas also been used in many fundamental concepts: the verification of the no-slip boundary condition, hydrodynamic stability [9], higher and lower order bifurcation phenomena and flow structures [10,59,60], but also in the field of combustion [61-63], drag reduction [64-67], magnetohydrodynamics in order to study e.g. the MRI [68-72], astrophysics to study Keplerian flow in accretion discs [73-76], rotating filtration in order to extract plasma from whole blood [77-81], cooling of rotating machinery [82], flows in bearings, the fundamentals of high Reynolds number flows [83-90], and as a catalytic and plasmapheretic reactor [91-93]. Hence the Taylor-Couette geometry is a versatile geometry in physics, engineering, and beyond.

Despite its ubiquity in industrial applications and our daily life, boiling and cavitation, collectively constituting rapid liquid-to-vapor phase transitions (as opposed to slow evaporation), is a field hitherto unexplored in Taylor-Couette. Withal, boiling has almost exclusively been an engineering subject. However, the nucleation of the bubble, the interaction of the gas and vapor with the liquid (absorption, evaporization, and heat transfer), the fluid dynamics of the vapor and gas inside the nucleating bubble and the fluid dynamics of the liquid around the oscillating, moving, and growing bubbles are hardly touched in the field of physics and only "quasi-static boiling" is considered, i.e. the statistics and thermodynamics of phase transitions. Nevertheless, above mentioned rapid features of the phase transitions-boiling - are certainly interesting and involve practically relevant physics that is still in need of deep and fundamental understanding. Boiling can be studied at the surface and in the bulk [94, 95], and can focus on a single vapor (nano)bubble [96, 97], or the collective effects of multiple vapor bubbles. The complexity and intractability of the boiling process is very well exemplified by the so-called boiling curve, which shape and explanation is far from trivial [98-101], and still lacks detailed physical interpretation.

With the TC system introduced in this chapter we plan to study the collective dynamics of many vapor bubbles in the bulk [102]. The combination of vapor bubbles with turbulence complicates the phase transition: first, the present vapor bubbles provide nucleation sites for more boiling, second, the turbulence provides intermittent flow (with associated intermittent kinetic energy spikes), and third, turbulent flows have locally low pressure spots-all of which provide favorable conditions for boiling to initiate.

To trigger the liquid-to-gas phase transition we have to carefully control the kinetic and thermal energy of our working liquid. It is therefore necessary to have a closed geometry with precise boundary conditions for both the velocity (kinetic 
energy) and the temperature (thermal energy). The Taylor-Couette geometry is a good candidate to study the physics of boiling in a controlled way because we can control these boundary conditions, it has global energy and momentum balances[12], its geometry has many symmetries, and it is relatively easy to construct. Though our existing apparatus, the $\mathrm{T}^{3} \mathrm{C}[103]$, is able to accurately rotate and control the temperature within $0.1 \mathrm{~K}$, its rather large volume of $111 \mathrm{~L}$ makes an experiment with low-boiling-point fluorinated liquids like FC-87 or FC-3284 a costly endeavour. The presented apparatus is smaller compared to the $\mathrm{T}^{3} \mathrm{C}$ facility, but is still capable of attaining a very turbulent flow of $\operatorname{Re}=\omega_{i} r_{i}\left(r_{o}-r_{i}\right) / v=\mathscr{O}\left(10^{6}\right)$ and operates in the so-called ultimate regime [12] so the study of nucleation of vapor bubbles inside Taylor vortices can be performed. For more details about the ultimate regime, and an introduction in Taylor-Couette flows in the ultimate regime in context of the applicability of the Taylor-Couette geometry we refer the reader to the review article of Grossmann, Lohse, and Sun[11]. The classical (non-ultimate) regime, with its many different flow structures, is well described by the pioneering work of Andereck et al. [10] and by a recent review article by Fardin et al. [104]. Other setups for high Re flows have been created [105-108], but either do not feature the very precise rotation, and cooling and heating methods that we aim for, or do not have completely transparent top and side cylinders, or do not feature an overflow channel that we need for vaporizing liquids.

The new setup is designed to study macroscopic boiling in the bulk of the working fluid. The improved design is based on the $\mathrm{T}^{3} \mathrm{C}[103]$, and includes accurate temperature control, and is dubbed the Boiling Twente Taylor-Couette (BTTC) facility. The system is able to accurately heat and cool both the inner and outer cylinder independently over a large temperature range of $0{ }^{\circ} \mathrm{C}-60^{\circ} \mathrm{C}$ which can be extended using special coolants rather than water. The temperature of the working fluid is closely monitored at three locations on the inner cylinder and at two locations at the outer cylinder. Section 4.2.3 describes the temperature control in more detail. The inner and outer cylinder have a maximum rotation rate of $f_{i}= \pm 20 \mathrm{~Hz}$ and $f_{o}= \pm 10 \mathrm{~Hz}$, respectively. The maximum Reynolds numbers are

\begin{tabular}{|r|c|c|c|}
\hline & Water & FC-3248 & FC-87 \\
\hline$f_{i}$ & \multicolumn{3}{|c|}{ $\pm 20 \mathrm{~Hz}$} \\
\hline$f_{o}$ & \multicolumn{3}{|c|}{ $\pm 10 \mathrm{~Hz}$} \\
\hline $\mathrm{Re}_{i}=\omega_{i} r_{i}\left(r_{o}-r_{i}\right) / v$ & $2.8 \times 10^{5}$ & $6.7 \times 10^{5}$ & $10.5 \times 10^{5}$ \\
\hline $\mathrm{Re}_{o}=\omega_{o} r_{o}\left(r_{o}-r_{i}\right) / v$ & $2.0 \times 10^{5}$ & $4.7 \times 10^{5}$ & $7.3 \times 10^{5}$ \\
\hline $\mathrm{Re}=\mathrm{Re}_{i}+\mathrm{Re}_{o}$ & $4.8 \times 10^{5}$ & $11.4 \times 10^{5}$ & $17.8 \times 10^{5}$ \\
\hline
\end{tabular}

Table 4.1: Current maximum rotation rates and their corresponding Reynolds number of the BTTC around room temperature. 
tabulated in 4.1, and more details about the driving mechanism can be found in section 4.2.2. The inner cylinder comprises three, vertically stacked, cylinders. The top and bottom inner cylinders can be removed in order to have vertical gaps of similar width as the radial gap. The entire outer cylinder and the top plate rotate together and are completely transparent, and do not have obstructing tension bars like the $\mathrm{T}^{3} \mathrm{C}[103]$. These transparent parts allow for detailed investigation of local quantities using optical methods like particle image velocimetry (PIV), laser Doppler anemometry (LDA), and particle tracking velocimetry (PTV), and regular (high-speed) imaging. An overflow channel that can be used during rotation of either cylinder is one of the unique features of the presented setup, and will be used to capture the expanding or boiling fluid and vapor.

\subsection{System description}

\subsubsection{General}

The system has two independently rotating cylinders confining a liquid in the measurement gap, see figure 4.1 for a cross section of the BTTC. The inner cylinder is made of copper and is chrome plated and has an outside radius $r_{i}=75 \mathrm{~mm}$. The outer cylinder is made from Poly(methyl methacrylate) (PMMA) and has an inside radius of $r_{o}=105 \mathrm{~mm}$. The radial gap is therefore $d=r_{o}-r_{i}=30 \mathrm{~mm}$, and the radius ratio is $\eta=r_{i} / r_{o}=0.714$. The gap has a height of $L=549 \mathrm{~mm}$, giving a measurement volume of $V=\pi\left(r_{o}^{2}-r_{i}^{2}\right) L=9.31 \mathrm{~L}$, and an aspect ratio of $\Gamma=L / d=18.3$. The middle inner cylinder, see Fig. 4.1 , has a height of $L_{\text {middle }}=489 \mathrm{~mm}$, while the height of the top and bottom inner cylinders are $L_{\text {top } / \text { bottom }}=26 \mathrm{~mm}$. The gap between each of the cylinders and between the top and the bottom are $\delta=2 \mathrm{~mm}$. In

\begin{tabular}{|c|c|c|c|c|c|}
\hline & BTTC & \multicolumn{3}{|c|}{$\mathrm{T}^{3} \mathrm{C}$} \\
\hline$L(\mathrm{~mm})$ & 549 & \multicolumn{4}{|c|}{927} \\
$L_{\text {middle }}(\mathrm{mm})$ & 489 & \multicolumn{4}{|c|}{536} \\
$L_{\text {top } / \text { bottom }}(\mathrm{mm})$ & 26 & \multicolumn{4}{|c|}{193.5} \\
$r_{i}(\mathrm{~mm})$ & 75 & \multicolumn{4}{|c|}{200} \\
$r_{o}(\mathrm{~mm})$ & 105 & 279 & 260 & 240 & 220 \\
$d=r_{o}-r_{i}(\mathrm{~mm})$ & 30 & 79 & 60 & 40 & 20 \\
$\eta=r_{i} / r_{o}$ & 0.714 & 0.716 & 0.769 & 0.833 & 0.909 \\
$\Gamma=L / d$ & 18.3 & 11.68 & 15.45 & 23.18 & 46.35 \\
$V(\mathrm{~L})$ & 9.31 & 111 & 80.4 & 51.3 & 24.5 \\
\hline
\end{tabular}

Table 4.2: Geometric dimensions of the BTTC and the $\mathrm{T}^{3} \mathrm{C}$ facility [103]. The outer cylinder of the $\mathrm{T}^{3} \mathrm{C}$ can be varied and all flow options are listed. 


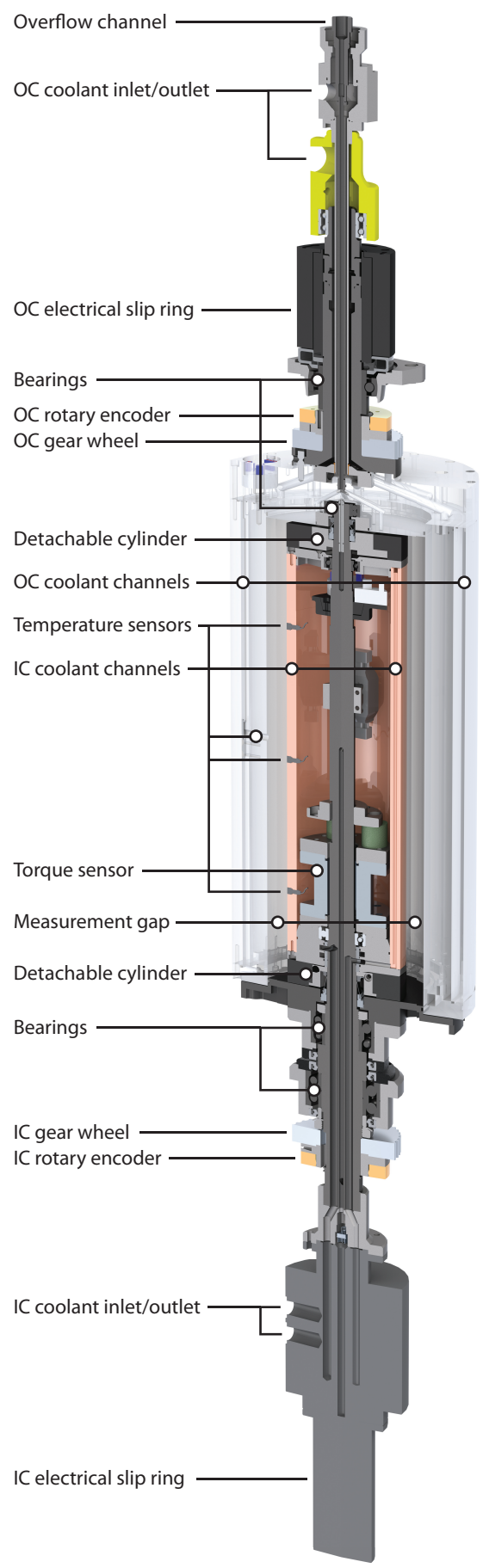

Figure 4.1: Vertical cross section of the BTTC. The inner cylinder is made of copper and is designated as IC. The outer cylinder, designated as $\mathrm{OC}$, is made of PMMA. All the steel that is in contact with water is made of 316 grade stainless steel to prevent corrosion, except for the axis of the inner cylinder which is machined from Duplex 1.4462 stainless steel. 
addition, top and bottom inner cylinders have been made that leave no gap between the inner cylinders. Table 4.2 summarizes the relevant dimensions. The top plate and the outer cylinder are machined from transparent PMMA by Hemabo (Hengelo, The Netherlands) and are heat treated to relieve internal stresses in the material, while the bottom plate is machined from corrosion-resistant 316 stainless steel. These end plates and the outer cylinder are fixed together and rotate in unison. The driving axis of the inner cylinder is machined from Duplex 1.4462 stainless steel, while the end plates of the middle inner cylinder, as well as the holders for the detachable cylinders are made from 316 stainless steel. The driving as well as the ingress and egress of the coolant of the inner cylinder is at the bottom, while these are situated at the top for the outer cylinder, see also Fig. 4.1. The bearing in the top-plate is sealed using a mechanical seal (AES Seal, type: B052-20), and the measurements gap is sealed at the bottom by another mechanical seal (AES Seal, type: B052-40), sealing the interface between the outer and inner cylinder. Both mechanical seals are of the carbon-ceramic type. At the top of the middle inner cylinder a V-ring seal (type: V-25S) is employed. The top and bottom inner cylinders are not hollow and therefore do not require sealing. The bearings of the driving axis of the inner cylinder, and the bearings of the bottom plate of the outer cylinder are protected against dust and water using seals (Simmering, BAUX2 NBR).

\subsubsection{Driving}

The inner (outer) cylinder was designed to rotate at the current maximum rotational velocity of $20 \mathrm{~Hz}(10 \mathrm{~Hz})$, although there is room to increase those velocities

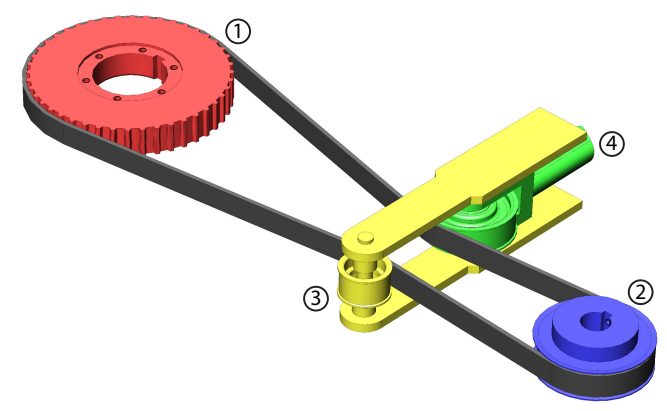

Figure 4.2: Inner (lower) pulley assembly. The red pulley (1) is attached to the axis driving the inner cylinder, the blue pulley (2) is attached to driving motor. In yellow (3) is the idler pulley, while the belt tensioner (green, 4) can be moved inwards/outwards to adjust the tension. Keyed joints and toothed pulleys do not allow for differential rotation between the belt, pulley, and their respective axes. 
if the vibrations in the system, forces on the outer cylinder, and heating up of the slip rings allow for that. The inner cylinder has a gear ratio of $44: 22=2: 1$ while the outer cylinder has a ratio of $44: 18 \approx 2.44: 1$. Both the inner and outer cylinder are driven by $16 \mathrm{~mm}$ toothed belts and pulleys from Mädler, Germany (type: AT10), and each belt has an idler pulley and a belt tensioner to ensure the belt neither vibrates nor skips any teeth. Figure 4.2 shows the pulley assembly of the inner cylinder, the assembly of the outer cylinder is nearly identical. All pulleys feature keyways and all driving axes feature keyseats preventing relative rotation. Both cylinders are driven by servomotors with internal feedback resolvers from Beckhoff (type AM8543-1H00 for the IC, type AM8543-1E00 for the OC) and have a rated power of $2.57 \mathrm{~kW}$ and $1.39 \mathrm{~kW}$ and maximum rotation rate of $5000 \mathrm{rpm}$ and $2500 \mathrm{rpm}$ for the inner and outer cylinder engine, respectively.

Both servomotors are driven by a 2-channel digital compact servo drive from Beckhoff (type AX5206), and each of the outputs of this drive is filtered by a AX2090-MD50-0012 motor choke from Beckhoff. The drive is outfitted with a brake resistor (Beckhoff, up to $600 \mathrm{~W}$ dissipation) to speed up the braking. The drive is controlled through high-speed EtherCAT system communication, and is connected to the other programmable logic controllers (PLCs). More about system control can be found in section 4.2.4.

Magnetic angle encoders are used to independently measure the angle of the shafts, and, by differentiation, the rotation rates. Each shaft is outfitted with an

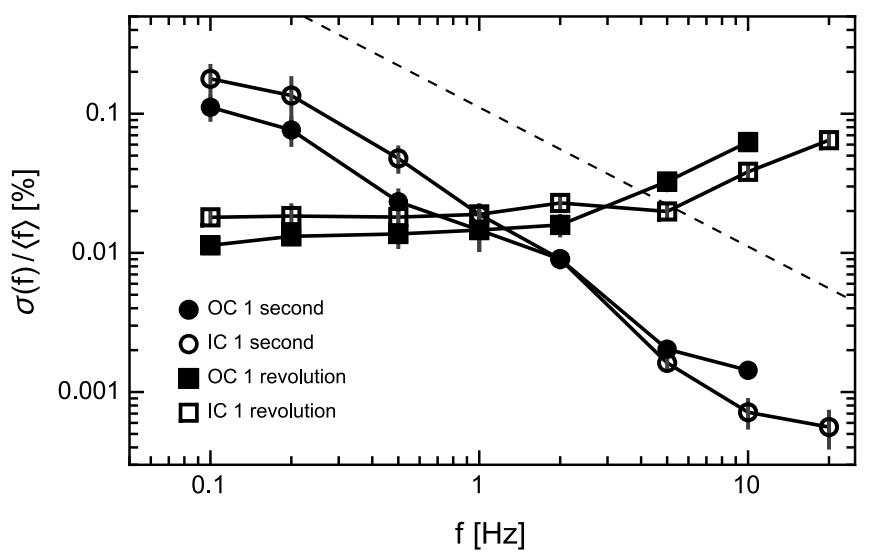

Figure 4.3: Standard deviation of the inner cylinder (IC) and outer cylinder (OC) rotation rates expressed as a percentage of the average rotation rate. We extract the mean velocity over a period of 1 second or the equivalent time of 1 revolution. The dashed line shows the maximum error that can originate from the angular resolution of the magnetic encoders for the case in which we average over 1 second. The slope of this line is -1 . 
ERM200-series angle encoder from Heidenhain (Schaumburg, USA) with a bore of $70 \mathrm{~mm}$, and a line count of 900 , giving an angular resolution of $0.4^{\circ}$ with an accuracy of $\pm 25^{\prime \prime}$. These magnetic encoders are connected to two incremental encoder interfaces by Beckhoff (type EL5101).

The performance of our driving system is checked by varying the rotation rate from $0.1 \mathrm{~Hz}$ to $20 \mathrm{~Hz}$ for the inner cylinder and from $0.1 \mathrm{~Hz}$ to $10 \mathrm{~Hz}$ for the outer cylinder. The angle was measured at an acquisition rate of $100 \mathrm{~Hz}$, and using a sliding linear fit the rotation rate is extracted for a duration of either 1 second or a period corresponding to 1 revolution. The standard deviation is then computed for these rotation rates, and normalized by the average rotation rates, see Fig. 4.3. The rotation rate always has a standard deviation smaller than $0.2 \%$ of the setpoint, and generally much better than this figure. Note that for measurements where the rotation angle is small (slow rotation speeds during a 1 second interval), the angular resolution of $0.4^{\circ}$ can cause errors. This maximum error caused by the angular encoder is indicated in Fig. 4.3, and is based on a measurement duration of 1 second. To exemplify: if we rotate at a frequency of $\frac{5}{9} \mathrm{~Hz}$ and we do a measurement over 1 second, the cylinder should have rotated $200^{\circ}$. The angular resolution of $0.4^{\circ}$ means that both the initial and final positions are both at most $0.2^{\circ}$ off from their actual values, and the error caused by the finite resolution is therefore at most (worst-case scenario) $\left(0.2^{\circ}+0.2^{\circ}\right) / 200^{\circ}=0.2 \%$. We find that the deviations for the 1 second interval measurements are below this limit indicating that the error is most likely caused by the finite resolution of the measurement in addition to the error introduced by the engine. We compare the performance from the current setup with the $\mathrm{T}^{3} \mathrm{C}$ [103] and the new Taylor-Couette setup in Göttingen[108] for which the authors compare the standard deviation for the case of $f=5 \mathrm{~Hz}$ over a single cylinder revolution. They found that they were both $0.1 \%$. Our current setup has values of $0.02 \%$ and $0.03 \%$ (at $5 \mathrm{~Hz}$ ) for the inner and outer cylinders, respectively. These standard deviations increase to about $0.06 \%$ for the maximum rotation rates of both cylinders.

\subsubsection{Temperature}

To allow for experiments with a very precise control of the temperature we have chosen to cool/heat the working fluid from the inner cylinder as well as from the outer cylinder in separate cooling circuits. In order to maximize the cooling power, the inner cylinder has been machined of copper, and has been chrome plated in order to prevent corrosion. The inner cylinder has 12 parallel channels (and 12 return channels) for the coolant, making use of countercurrent heat exchange to lower thermal inhomogeneities, see also Fig. 4.4. The outer cylinder is machined from PMMA (Hemabo, Hengelo, The Netherlands) and it therefore has a poor thermal conductivity, despite that, we also cool the outer cylinder in a second cooling 


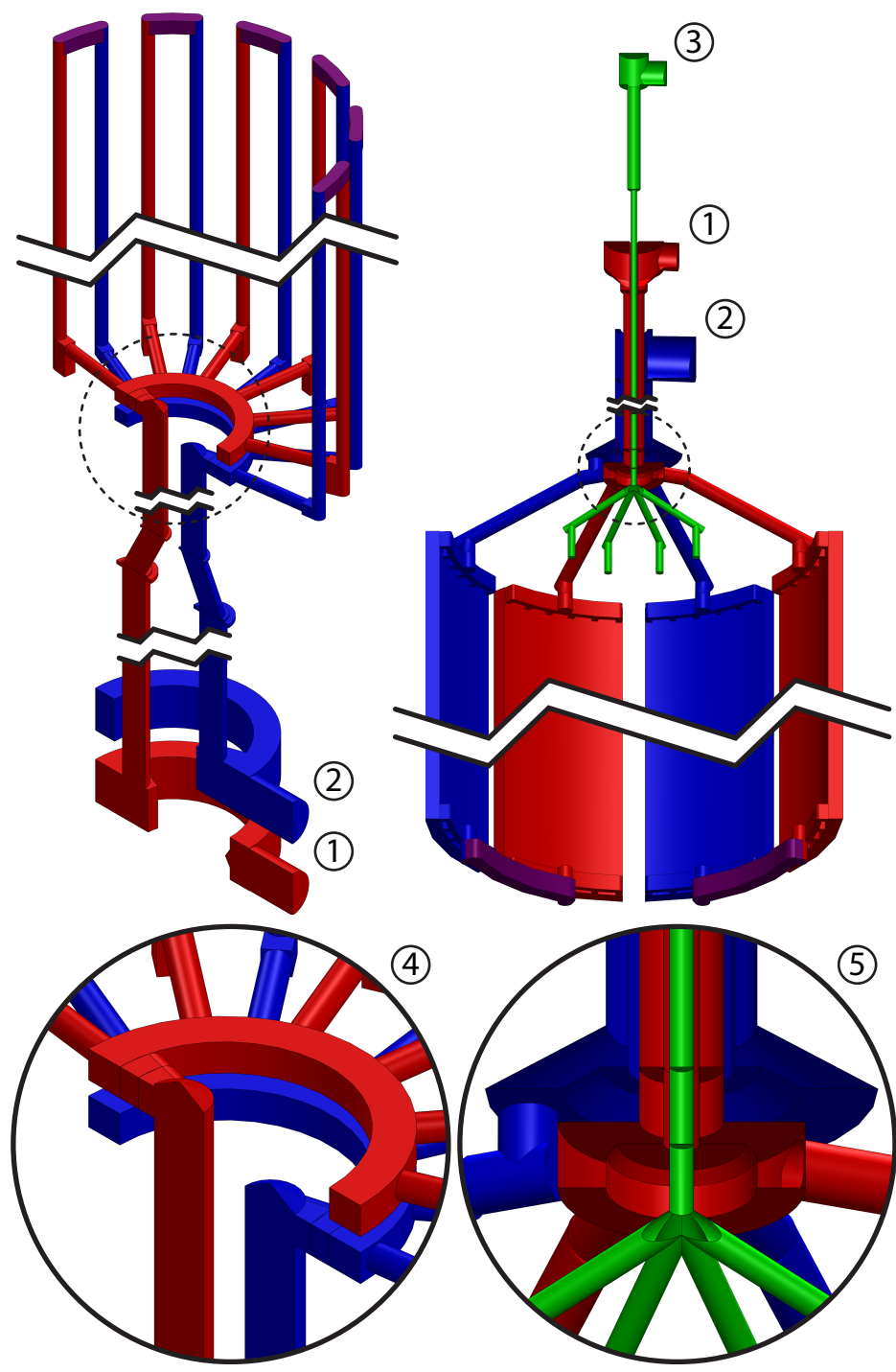

Figure 4.4: Left(right): Section view of the cooling channels of the inner(outer) cylinder. Coolant enters the cooling circuit at 1 where the fluid goes through a rotary union to the rotating frame. The coolant is split up in to 12(4) parallel streams inside the inner(outer) cylinder, see the detail view 4(5) at the bottom. The coolant then flows axially, enters the purple 'bridges' and returns in the other 12(4) parallels channels, and is again combined at $4(5)$. The returning coolant continues to flow axially (and concentrically for the outer cooling circuit) to the entering coolant. The coolant leaves the coolant circuit through a rotary union at 2. The apparatus is equiped with an overflow channel, shown in green. A mix of vapor, gas, and liquid can exit the system through the rotary union at 3. 


\begin{tabular}{|l|r|c|c|}
\hline Case & $f_{i}[\mathrm{~Hz}]$ & $\left\langle\sigma_{t}(\mathrm{~T})\right\rangle_{n}[\mathrm{~K}]$ & $\sigma_{n}\left(\langle T\rangle_{t}\right)[\mathrm{K}]$ \\
\hline Low & 1 & 0.013 & 0.056 \\
Low & 2 & 0.012 & 0.055 \\
Low* & 5 & 0.013 & 0.052 \\
Low & 10 & 0.015 & 0.049 \\
Low & 20 & 0.014 & 0.054 \\
High & 1 & 0.017 & 0.088 \\
High & 2 & 0.019 & 0.042 \\
High & 5 & 0.027 & 0.051 \\
High & 10 & 0.021 & 0.062 \\
High & 20 & 0.021 & 0.061 \\
\hline
\end{tabular}

Table 4.3: Standard deviation of the local temperatures for a low setpoint around $20^{\circ} \mathrm{C}$ and a high setpoint around $50^{\circ} \mathrm{C}$. The duration of each of the recordings is one hour long. $\sigma(\ldots)$ denotes standard deviation, $\langle\ldots\rangle$ denotes averaging, and the subscript $n(t)$ denotes that the operation is over the different sensors(over time). The time evolution for the entry with a star is shown in Fig. 4.5.

circuit that goes through the driving axis of the outer cylinder (at the top) in order to ensure that the thermal boundary is always identical. The outer cylinder has 4 parallel streams where the coolant flows axially around the working fluid inside the measurement gap. Four rotary unions provide the means of ingress to and egress from the cooling circuits (bottom: GAT mbH, Germany, type Rotocombi SW65-W2-HM, top: Deublin, 357-130-235 and a custom-made rotary union). An additional rotary union (Deublin, type: 1115-000-205) is installed at the top, and allows for the system to overflow such that the expanding liquid, gas, or vapor can be captured, see the green channels in Fig. 4.4. The cooling circuit of the inner and outer cylinder are connected to two refrigerated/heating circulators by Julabo (type: FP50-HL) with a maximum cooling capacity $0.9 \mathrm{~kW}$, a heating capacity of $2 \mathrm{~kW}$, a temperature stability of $0.01 \mathrm{~K}$, and a working temperature range of $-50{ }^{\circ} \mathrm{C}$ $-200^{\circ} \mathrm{C}$. Our temperature range is however limited by the glass transition temperature of PMMA which can be as low as $82^{\circ} \mathrm{C}$, and the heaters are therefore limited to a maximum of $60^{\circ} \mathrm{C}$ to ensure full strength of the outer cylinder during rapid rotation while having a large safety margin. The Julabo coolers/heaters now have water as their coolant, in order to reach sub-zero temperatures for other types of experiments one has to replace the coolant by e.g. a glycol based or glycerol based coolant.

The inner cylinder embeds three PT100 temperature sensor at different heights to monitor the temperature of the working fluid, while the outer cylinder embeds another two sensors to check the temperature. The five PT100 sensors are each connected to a precalibrated signal conditioner IPAQ-H ${ }^{\text {plus }}$ from Inor (Malmö, 


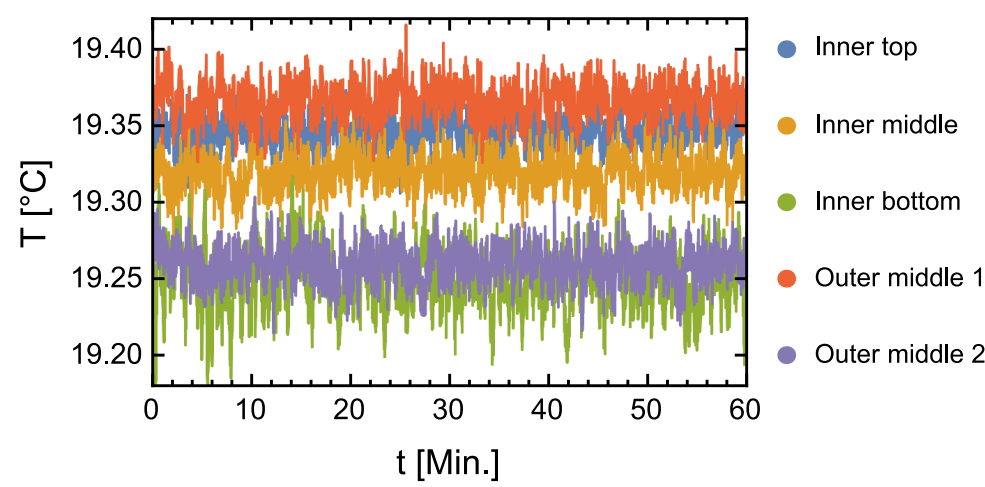

Figure 4.5: Time evolution of the temperature for the low temperature setpoint for the case of $f_{i}=5 \mathrm{~Hz}$, see the starred row in table 4.3. The colors correspond to different sensors in either the inner or outer cylinder, see the legend on the right.

Sweden) inside the inner or outer cylinder. This combination of sensor and signal conditioner results in a specified absolute temperature accuracy of $0.1 \mathrm{~K}$ and for a relative temperature accuracy of $0.01 \mathrm{~K}$. The three sensors inside the inner cylinder are mounted flush (at $z=0.22 \mathrm{~L}, z=0.49 \mathrm{~L}$, and $z=0.76 \mathrm{~L}$ ), are thermally isolated from the copper, and are in direct contact with the working fluid. The two sensors in the outer cylinder are also mounted flush and are both located at $z=0.53 \mathrm{~L}$ and are positioned $180^{\circ}$ apart for balancing reasons. The current signals from the signal conditioners exit the system through slip rings, see Fig. 4.1, and are read out by 4-channel analog input terminals from Beckhoff (type EL3154).

The performance of our temperature control is checked by varying the rotation rate and the setpoint: a low temperature around room temperature and a high temperature around $50^{\circ} \mathrm{C}$. We tabulate the stability of the temperature in tab. 4.3. We find that the temperature stability is better than $0.03 \mathrm{~K}$, and generally better than $0.02 \mathrm{~K}$, see the $3^{\text {rd }}$ column of tab. 4.3. The temperature distribution inside the measurement gap ( $4^{\text {th }}$ column) is better than $0.1 \mathrm{~K}$, which is in correspondence with the absolute temperature accuracy of the sensors of $0.1 \mathrm{~K}$. As an example we show the time evolution of the temperature for the low temperature setpoint for the case of $f_{i}=5 \mathrm{~Hz}$, see Fig. 4.5. We do not find any systematic radial or height-dependence of the temperature due to the large cooling/heating surface (the entire inner and outer cylinder) combined with the excellent turbulent mixing causing a nearly homogeneous temperature inside the measurement gap, which provides an excellent playground to study the physics of boiling in the bulk. 


\subsubsection{Wiring and control}

A diagram of the BTTC wiring is shown in Fig. 4.6. Temperature and torque signals from the inner cylinder leave the rotating frame through a slip ring at the bottom made by GAT mbH (type: SRK 80/18, 18 channels). The temperature signals from the outer cylinder exit the system at the top via a through-bore slip ring (type: SRH 80180 FT 14, 14 channels) made by Gileon. The signals are digitalized by analog input modules (type EL3154 and EL3124) from Beckhoff. The communication to the Julabo temperature controllers are done through analog output modules (type: EL4004) from Beckhoff. The communication with all the DAQ and PLCs is done through the EtherCAT protocol (by Beckhoff Automation), which connects the computer and the Beckhoff modules and the communication

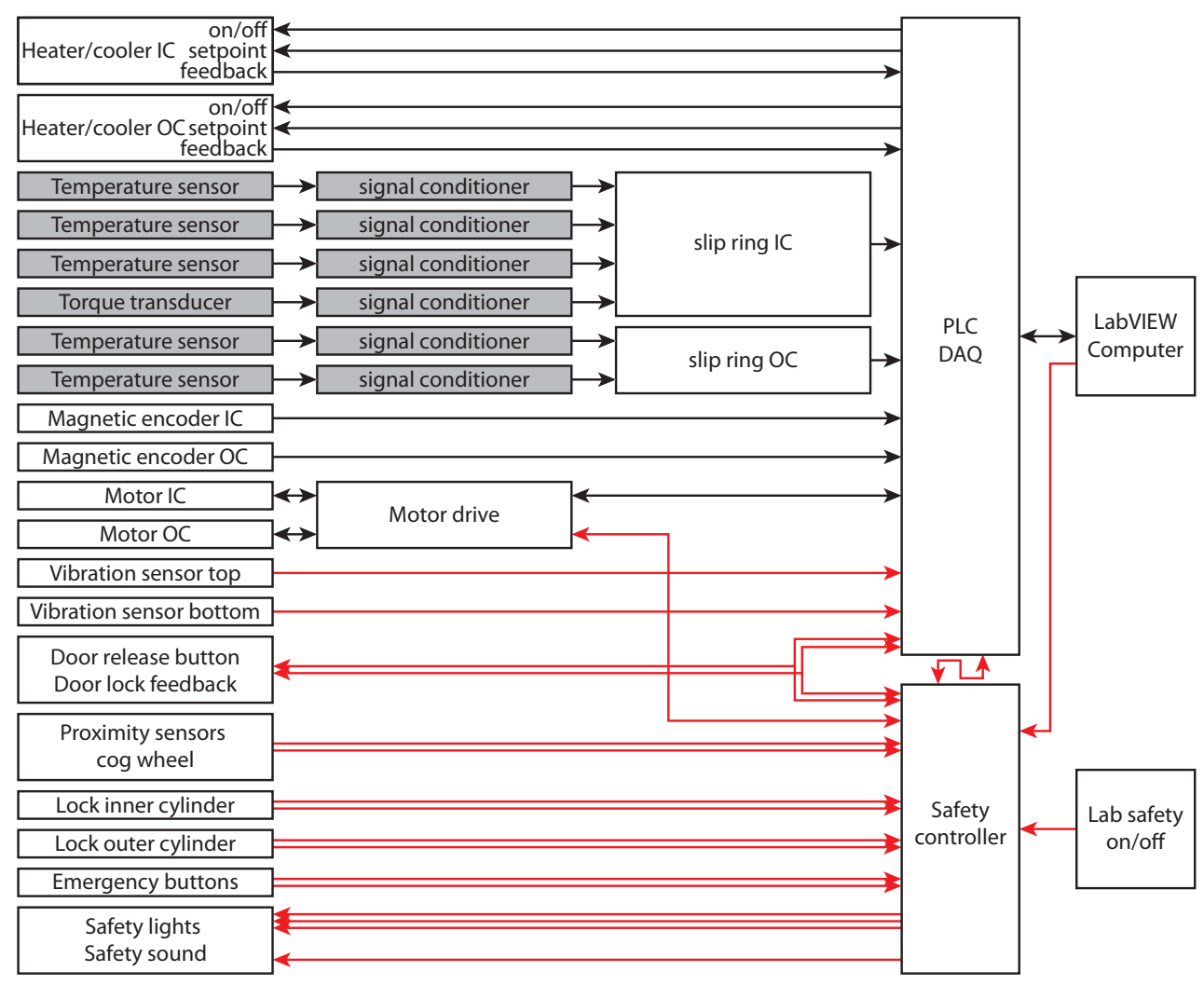

Figure 4.6: Sketch of the BTTC wiring. Grey components are embedded in the inner or outer cylinder. Red lines indicate that these control safety-related aspects of the setup. PLC stands for programmable logic controller, DAQ for data acquisition, IC for inner cylinder, and OC for outer cylinder. 
to the safety module from Omron (type G9SX-LM244-F10). See section 4.2.5 for more information about safety. All the data acquisition and control of the coolers/heaters and motors are performed at a rate of $100 \mathrm{~Hz}$ by the computer. The computer runs a LabVIEW (National Instruments) program by which the end user can control the various peripherals and save all the data. The LabVIEW software allows the end user to e.g. make a continuous velocity ramp from an initial rotational velocity to a final rotational velocity over a certain amount of time, for both cylinders individually. Also tabulated entries can be imported. Each entry will be performed sequentially and can contain different rotational velocities, cooling temperatures, filename for saving et cetera. These automated features minimize operational errors while maintaining high safety standards, see section 4.2 .5 for more about safety.

\subsubsection{Safety features}

The systems employs a wide variety of safety features to allow for a safe operation of the apparatus. The LabVIEW software continuously monitors the vibrations using vibration sensors by Sensonics Ltd (type: PZDC-58K00110, 0-15 mm/s range), mounted at the top and bottom bearings, as well as the temperature at five different locations. If one of these vibrations or one of these temperatures exceed their threshold (safety limit) for a set duration the system is brought to a halt and the coolers/heaters are switched off. Each of the cylinders can be mechanically locked. These locks can be in a 'lock' position or a 'free' position, both are actively detected using limit switches by Eaton (type: LSE-11). The signals for each pair of these sensors go through XOR gates; the entire system will only operate if the output of these gates are both high. If the 'lock' position is detected the system will ensure that the respective motor cannot be operated in order to prevent damage to belts, engines, and pulleys. The bottom plate of the outer cylinder has toothed edges which are detected by two proximity sensors. These proximity sensors by Omron (type: M8 shielded) are positioned and the size of the teeth is chosen in such a way that at least one of the sensors always detects a metal tooth. The signals of both sensors go through an OR gate, and only if this output is high the system is allowed to operate. This protective feature ensures that the outer cylinder is present during operation. The entire system is housed inside 4 metal frames with strong, polycarbonate windows. The front panel has two doors to access the apparatus. The software is configured such that the system is allowed to rotate only if the doors are closed, and the system will halt if the doors are forced open during operation. The system can be halted in the case of an emergency by the safety module, or indirectly by the LabVIEW software (either automatically or manually), but in addition the system also has three emergency buttons that are within the vicinity of the operator. The buttons all cause the power of the engines to be inter- 
rupted. Moreover, the system is also connected to the safety features of the laboratory. In case the apparatus is operated without the outer polycarbonate frames (this might be necessary if more space around cylinders is required for a laser or camera equipment), the door of the lab cannot be opened from the outside while the system is in rotation-minimizing the risk that someone can come in direct contact with the outer cylinder while the system is operational. Running the apparatus without front panel and doors will enable another layer of security: the system will make a beeping sound for a few seconds before the rotation is started. This last feature ensures that everyone is aware that the system is about to rotate and stays away from the rotating parts. The front panel (or a separate pole that is needed for operating the system without front panel) also features three lights and a sound alarm that indicate the current status: ready to operate (green light), operating (orange light), system halted due to the safety mechanisms (red light + sound signal), see also the rendering of the entire apparatus in Fig. 4.7.

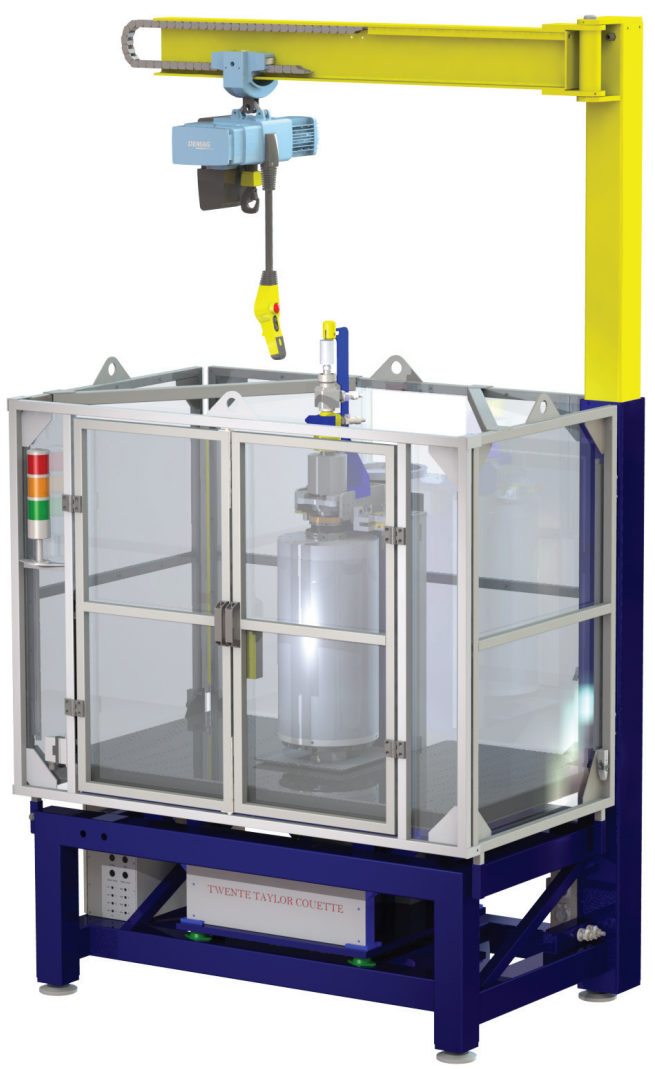

Figure 4.7: Rendering of the new BTTC apparatus. 


\subsubsection{Other details and features}

The system is filled from the bottom of the apparatus through two filling channels embedded inside the bottom plate of the outer cylinder. The system can overflow through a special channel at the top of the apparatus; the liquid of the measurement gap is connected to 8 channels above the top inner cylinder where the expanding liquid can escape the system, see Fig. 4.1 "Overflow channel", or see the green channels in Fig. 4.4. The system can be emptied at the bottom through the same channels as for filling. Apart from a transparent outer cylinder and top plate with cooling channels we also have an outer cylinder and top plate that have no channels and no temperature sensors and are also completely transparent. One can attain clearer images using these parts because the number of interfaces is reduced, which might be imperative for e.g. high resolution PIV measurements through the top plate or the outer cylinder. The inner cylinder also embeds a hollow flanged reaction torque transducer (Althen $\mathrm{GmbH}$, type: 01167-051) that can sense the torque that is needed to drive the inner cylinder. The inner cylinder is mounted on bearings to the driving axis, and the torque sensor connects the driving axis with the inner cylinder. The torque sensor senses the torque on the entire copper inner cylinder, having a height of $L=489 \mathrm{~mm}$, see also figure 4.1. The inner cylinder has several seals to prevent water from leaking into it. It remains to be seen whether or not the shear of the flow is high enough such that the friction of all these seals can be considered as negligible (in order to solely get the fluid torque signal). The frame features an integrated swinging crane (Demag, type: DS-Pro 5-500 with steplessly variable controller) with a jib that can be used to assemble and disassemble the system with ease. The integrated crane is telescopic and can be lowered to provide clearance to move it in and out of the lab, see also Fig. 4.7. A custom-made optical table (dimensions: $1500 \mathrm{~mm} \times 900 \mathrm{~mm} \times 60 \mathrm{~mm}$, made by Thorlabs) installed around the Taylor-Couette cylinders provides the means to easily mount cameras, optical elements, and other equipment, see Fig. 4.7. The optical table, the cylinders, the bearings, the engines, and all the supporting structure are mounted inside the main frame on vibration isolators made out of rubber. The vibrations of the Taylor-Couette cylinders, inner frame, and the optical table are isolated with respect to the outer frame and the windows.

\subsection{Example of flow measurement}

The BTTC apparatus features a fully transparent top plate allowing for easy flow visualizations in the $\theta-r$ plane. This feature is exemplified by performing PIV measurements at three rotational velocities of the inner cylinder $\left(f_{i}=5 \mathrm{~Hz}, f_{i}=10 \mathrm{~Hz}\right.$, and $f_{i}=20 \mathrm{~Hz}$ ). The fluid is seeded with $1-20 \mu \mathrm{m}$ tracer particles, the fluid (water) is illuminated using a laser sheet around mid height at $z=0.49 \mathrm{~L}$. Based on 

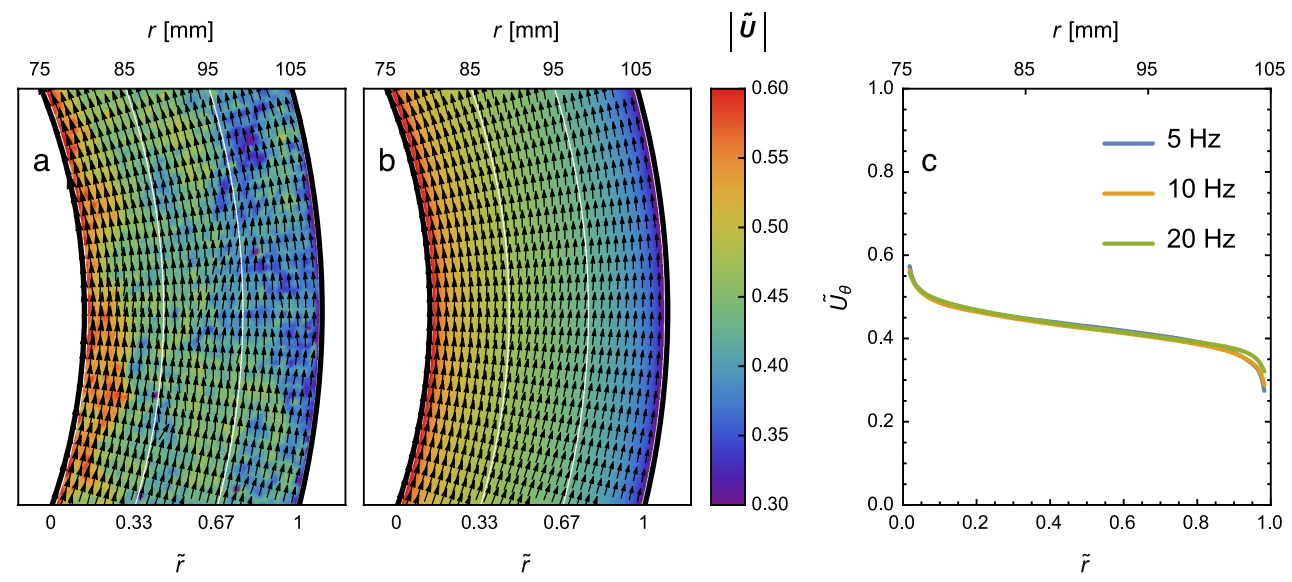

Figure 4.8: (a) Instantaneous flow field for a rotation of $f_{i}=10 \mathrm{~Hz}$ and stationary outer cylinder. Color indicates the magnitude of the vectors. We define $\tilde{r}$ as the normalized distance between the cylinders: $\tilde{r}=\left(r-r_{i}\right) / d$, and the normalized velocity as $|\tilde{\mathbf{U}}|=$ $|\mathbf{U}| /\left(2 \pi f_{i} r_{i}\right)$, and $|\ldots|$ denotes the Euclidean norm. (b) Averaged flow field for the same rotation velocities. (c) Radially binned, and temporally and azimuthally averaged azimuthal velocity profiles normalized with the driving velocity $\left(\tilde{U}_{\theta}=U_{\theta} /\left(2 \pi f_{i} r_{i}\right)\right)$ for $f_{i}=5 \mathrm{~Hz}$, $f_{i}=10 \mathrm{~Hz}$, and $f_{i}=20 \mathrm{~Hz}$, with Reynolds numbers $\operatorname{Re}_{i}=0.7 \times 10^{5}, \operatorname{Re}_{i}=1.4 \times 10^{5}$, and $\operatorname{Re}_{i}=2.8 \times 10^{5}$, respectively. Due to insufficient optical resolution we are unable to measure details of the boundary layer accurately in this measurement, but with modified optics focusing on the boundary layers this would be possible, too.

the particle size and Kolmogorov microscales from Lewis et al.[83] we estimate the particle response time $\tau_{p}$ and the Kolmogorov time scale $\tau_{\eta}$. The highest possible Stokes number for our particles $(20 \mu \mathrm{m}$ particles at the highest rotation speed of $f_{i}=20 \mathrm{~Hz}, \rho_{p}=1190 \mathrm{~kg} / \mathrm{m}^{3}$ as compared to $\rho_{f}=1000 \mathrm{~kg} / \mathrm{m}^{3}$ around $20^{\circ} \mathrm{C}$ ) gives $\mathrm{St}=\tau_{p} / \tau_{\eta} \approx 5 \times 10^{-4}$. The particles therefore faithfully follow the flow, and can be considered as tracer particles. The laser used is a dual head Nd:YLF laser (Litron, type: LDY303PIVHE). The flow is imaged using an sCMOS camera with a resolution of 5.5 megapixel (PCO, Germany) from the top via a front surface mirror mounted at $45^{\circ}$. One thousand frames are recorded (with a maximum frame rate of $50 \mathrm{~Hz}$ ) for each measurement which provides sufficient averaging to become statistically stationary within $1 \%$. We perform so-called dual frame PIV measurements such that each pair of laser pulses provide two recorded images, which will be processed to a single PIV velocity vector field. With the maximum frame rate of $50 \mathrm{~Hz}$ of the camera this results in 25 PIV vector fields per second. We perform standard PIV algorithms with adaptive interrogation window size to compute the vector fields. The output of the algorithm are vector fields of $160 \times 135$ vectors. The high resolution set of vectors are linearly interpolated and a small set of vectors are shown in 
a polar grid, see figure 4.8 .

Figure $4.8 \mathrm{a}$ shows the instantaneous velocity field around mid-height $(z=$ $0.49 L$ ) for the case of $f_{i}=10 \mathrm{~Hz}$, corresponding to $\operatorname{Re}_{i}=\omega_{i} r_{i}\left(r_{o}-r_{i}\right) / v=1.4 \times 10^{5}$. The colormap shows the magnitude of the velocity (in the $\theta-r$ plane). The flow has been normalized by the rotation speed of the inner cylinder. Because of the high Reynolds numbers we are unable to directly measure the velocity boundary layers - the boundary layers are too sharp and our interrogation window too big to faithfully measure the velocity. The main flow direction is very close to purely azimuthal flow; the radial velocity is relatively low, but is very important in order to get higher-than-laminar transport $\left(\mathrm{Nu}_{\omega}>1\right.$ in the terminology introduced in ref. [12]). Figure $4.8 \mathrm{~b}$ shows the averaged flow field for the same case as figure 4.8a and displays a flow that is nearly purely azimuthal. In figure $4.8 \mathrm{c}$ we plot the azimuthally and time averaged velocity profiles for all the three rotation rates. The velocity has been normalized by the velocity of the inner cylinder: $\tilde{U}_{\theta}=U_{\theta} /\left(\omega_{i} r_{i}\right)$. The boundary layers and the 'sharpening' of the boundary layers for increasing driving velocity (Reynolds number) is evident.

In addition to an outer cylinder which has cooling, also a completely transparent outer cylinder without cooling channels has been manufactured. The completely transparent top plate and outer cylinder without obstructing tension bars allows for direct flow visualization, even for the case of outer cylinder rotation. The flow is visualized in the $r-z$ plane by having a laser sheet that is coincident with this plane and that is illuminating tracer particles in said flow. The reflected light from the particles can then be recorded and small illuminated trajectories then visualize the flow direction. The apparatus was set to rotate with a rotation ratio of $a=-\omega_{o} / \omega_{i}=0.4$ and $f_{i}-f_{o}=3.5 \mathrm{~Hz}$. A high-resolution digital single-lens reflex camera from Nikon (type: D800E, 36 megapixel) with a $50 \mathrm{~mm}$ lens from Zeiss (type: Makro-Planar T* 2/50 ZF.2) was used to take a photo of the illuminated tracers, see figure 4.9. Pairs of vortices are marked on the left side. The formation of turbulent Taylor vortices can clearly be seen. The vortices have an aspect-ratio of $\xi=h /\left(r_{o}-r_{i}\right)=1.14$, which is in accordance with Ref. [109]. The bottom half of the system is (in a time-averaged sense) a mirrored version of the top half. 


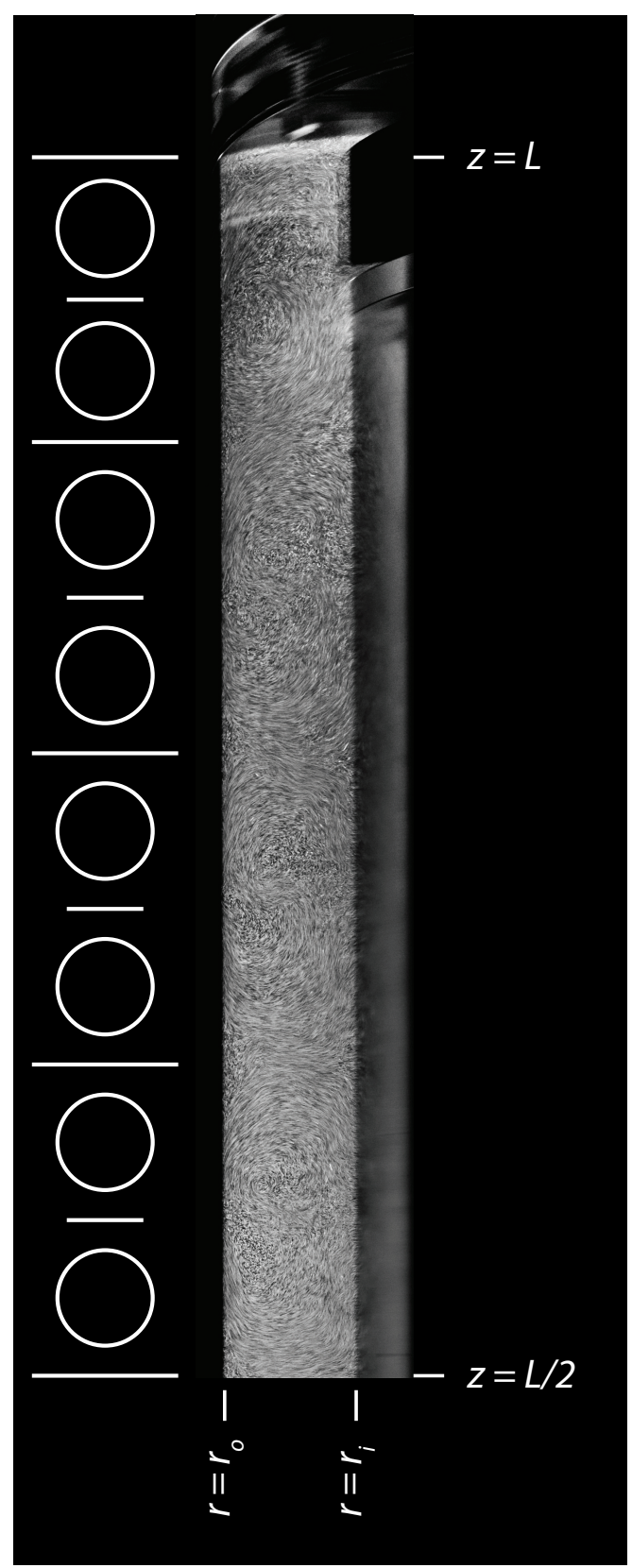

Figure 4.9: Visualization of the flow in the top half of the radial-axial plane. The flow is illuminated using a laser sheet in aforementioned plane and photographed from the side using a camera. One can clearly see the pattern of the light streaks visualizing the trajectory of tracer particles. Indications on the left show the placements of the vortex pairs. 


\subsection{Summary and outlook}

A new Taylor-Couette facility with independently rotating cylinders and precise temperature control has been constructed. The performance of the engines and angular encoders are tested and show very good performance for rotation rates ranging from $0.1 \mathrm{~Hz}$ to $20 \mathrm{~Hz}$. The apparatus is capable of reaching Reynolds numbers of up to $\operatorname{Re}_{i}=\omega_{i} r_{i}\left(r_{o}-r_{i}\right) / v= \pm 2.8 \times 10^{5}$ and $\operatorname{Re}_{o}=\omega_{i} r_{i}\left(r_{o}-r_{i}\right) / v= \pm 2 \times 10^{5}$ for the case of water, and up to $\mathrm{Re}_{i}= \pm 6.7 \times 10^{5}$ and $\operatorname{Re}_{o}= \pm 4.7 \times 10^{5}$ for the case of FC-3284 liquid. The setup can be heated and cooled from $0{ }^{\circ} \mathrm{C}$ to $60^{\circ} \mathrm{C}$ using water as the coolant and while maintaining a large safety factor. Using a different coolant we can possibly extent this temperature range from $-50^{\circ} \mathrm{C}$ to $80^{\circ} \mathrm{C}$ while maintaining a reasonable safety margin. The system has a wide variety of safety features to allow for safe operation of the apparatus. PIV measurements are performed and show that it is feasible to quantify the flow structures from the top. Imaging through the outer cylinder using a high-resolution camera allows us to easily measure the turbulent coherent structures in the vertical plane of the apparatus. The setup is able to cool and heat the outer and inner cylinder independently and was found to have a very good temperature stability. This temperature stability will be crucial in order to explore the boiling process in TC geometry, but before embarking on the exploration of phase transitions in Taylor-Couette flows the characterization of single phase flow in the presented geometry is required. Many open questions in the boiling process remain and we plan to study the nucleation process in this well-controlled turbulent environment, and the clustering dynamics of vapor clouds and its effects on nucleation. 


\section{5}

\section{Multiple states in highly turbulent Taylor-Couette flow $^{*}$}

The ubiquity of turbulent flows in nature and technology makes it of utmost importance to fundamentally understand turbulence. Kolmogorov's 1941 paradigm suggests that for strongly turbulent flows with many degrees of freedom and large fluctuations, there would only be one turbulent state as the large fluctuations would explore the entire higher dimensional phase space. Here we report the first conclusive evidence of multiple turbulent states for large Reynolds number $\operatorname{Re}=\mathscr{O}\left(10^{6}\right)$ (Taylor number $\mathrm{Ta}=\mathscr{O}\left(10^{12}\right)$ ) Taylor-Couette flow in the regime of ultimate turbulence, by probing the phase space spanned by the rotation rates of the inner and outer cylinder. The manifestation of multiple turbulent states is exemplified by providing combined global torque- and local-velocity measurements. This result verifies the notion that bifurcations can occur in high-dimensional flows (that is, very large Re) and questions Kolmogorov's paradigm.

${ }^{*}$ Published as: Sander G. Huisman, Roeland C. A. van der Veen, Chao Sun and Detlef Lohse, Multiple states in highly turbulent Taylor-Couette flow, Nat. Commun. 5, 3820 (2014). Experiments and analysis by SH and RVDV, writing by SH, supervision by CS and DL, discussion of the results and proofreading by everyone. 


\subsection{Introduction}

For macroscopic flows of water or air the Reynolds number is much larger than unity; the standard type of flow is therefore turbulent. A typical Reynolds number for a person walking is already $\mathscr{O}\left(10^{5}\right)$. For large airplanes the Reynolds number is $\mathscr{O}\left(10^{9}\right)$, for atmospheric currents it is $\mathscr{O}\left(10^{10}\right)$, and for ocean currents it is $\mathscr{O}\left(10^{11}\right)$. Reynolds numbers are even larger for astrophysical problems [110]. It is not possible to achieve these large Reynolds numbers in a lab-environment nor is it accessible by direct-numerical simulations (DNS). To extrapolate data from $\operatorname{Re}=\mathscr{O}\left(10^{6}\right)$ to the scales of our atmosphere or the ocean we must bridge 4-5 decades in Reynolds number, and even more for astrophysical applications [111]. While scaling laws exist that can predict the rough magnitude of these flows, they are rendered impractical if there is a flow-transition from a turbulent state at lower Re to another turbulent state at higher Re, or if multiple turbulent states can coexist at the same Re. To extrapolate to large scales we need to know whether there are transitions and whether multiple states can coexist in high Reynolds number flows. As an answer to this question, Kolmogorov's more than 70-year-old paradigm states that for large Reynolds numbers, flows would become 'featureless' due to the fact that the highly-dimensional phase space is explored in its entirety due to the large fluctuations of said flows [112, 113].

For Rayleigh-Bénard convection at low Rayleigh number (laminar-type boundary layers, $\mathrm{Ra}<10^{14}$ ), continuous switching between two different roll states, with different heat transfer properties, was found [114-116]. In this case, the turbulent fluctuations were large enough to overcome trapping in one turbulent state. In the case of high Rayleigh number (turbulent boundary layers), no multiple states have been observed in a single setup; only when the boundary conditions were changed one could trigger a transition to a different state [117, 118]. For von Kármán flow multiple turbulent states were found when driving it with impellors with curved blades [119-121]. These studies revealed the spontaneous symmetry-breaking and turbulent bifurcations in highly turbulent von Kármán flow up to Re $=10^{6}$. In spherical-Couette flow Zimmerman et al. [122] observed spontaneous switching between two turbulent states at fixed rotation rates. The presence of coherent structures at high Reynolds numbers in closed systems suggests that Kolmogorov's hypothesis [112, 113] is incomplete [119-122] and might need revisiting in order to apply to these flow systems. For Taylor-Couette multiple states are only observed for low Re (see e.g. ref. [10])—-the so-called classical regime, where the bulk is laminar or turbulent but the boundary layers are still of laminar type. Around $\operatorname{Re} \sim 10^{4}[123,124]$ the system transitions into the ultimate state $[105,118,125]$, in which the boundary layers are also turbulent [87], and where new scaling laws of the response parameters set in [84, 85, 107, 123, 126-129]. Historically, this ultimate regime was defined based on the scaling properties of the flow [125]. 
Consequently, it does not necessarily exclude the existence of multiple turbulent states. To correctly extrapolate to much higher Re it is crucial to know the characteristics of the turbulent state and the existence of other such states. In ref. [59] it was shown that for increasing Re the waves on top of the Taylor vortices become increasingly complex until only turbulent Taylor vortices are left. Lewis et al. [83] came to the same conclusion by plotting the velocity power spectra for increasing Re up to $5 \cdot 10^{5}$, and observed that the peaks gradually decrease in amplitude. They noted that turbulent Taylor vortices remained. On the other hand, the findings of Lathrop et al. [105] suggest that the Taylor-vortices are not present for Reynolds numbers beyond $1.2 \cdot 10^{5}$.

Here we demonstrate that roll structures remain for Taylor-Couette flow even in the ultimate regime up to at least $\operatorname{Re}=\mathscr{O}\left(10^{6}\right)$ and show that multiple states are even possible far beyond the transition into this ultimate regime.

\subsection{Results}

For TC flow $[84,85,107,123,127-129]$ and using the analogy of TC flow with Rayleigh-Bénard (RB) convection [14] it was found that the Taylor number Ta $=$ $\frac{1}{4}\left(\frac{1+\eta}{2 \sqrt{\eta}}\right)^{4}\left(r_{o}-r_{i}\right)^{2}\left(r_{i}+r_{o}\right)^{2}\left(\omega_{i}-\omega_{o}\right)^{2} / v^{2}$ is a very well suited parameter to describe the driving of the system [12]. Here $\omega_{i, o}=2 \pi f_{i, o}$ are the angular rotation rates and $v$ the kinematic viscosity. The response of the system is the torque required to sustain constant angular velocity or a 'Nusselt' number [12], $\mathrm{Nu}_{\omega}=\tau / \tau_{\text {lam }}$, which is the angular velocity flux nondimensionalised with the flux of the laminar, nonvortical, flow. This Nusselt number scales approximately as $\mathrm{Nu}_{\omega} \propto \mathrm{Ta}^{0.38}[84,85]$ around $\mathrm{Ta}=10^{12}$, which is interpreted as $\mathrm{Nu}_{\omega} \propto \mathrm{Ta}^{1 / 2} \cdot$ Log-corrections $[118,125]$.

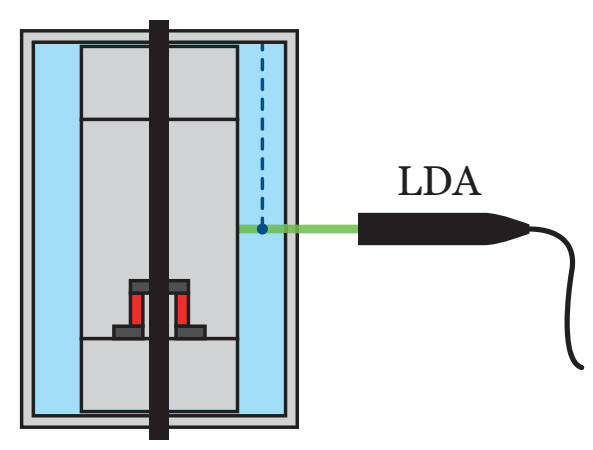

Figure 5.1: Experimental apparatus. Schematic of the cross section of the $T^{3} C$ [103]: the apparatus has been outfitted with a new coaxial torque transducer (shown in red), see the Methods section. The azimuthal velocity is probed at the middle of the gap and in the top half of the apparatus using laser Doppler anemometry (LDA). 
We now find with our new sensor with improved accuracy that the exponent is closer to 0.40 (results not shown), which is still consistent with the aforementioned interpretation. The Twente Turbulent Taylor-Couette facility $\left(\mathrm{T}^{3} \mathrm{C}\right)$ [103] was used for the experiments, see Fig. 5.1. The $\mathrm{T}^{3} \mathrm{C}$ [103] has an inner cylinder with an outer radius of $r_{i}=200 \mathrm{~mm}$, a transparent outer cylinder with inner radius $r_{o}=279 \mathrm{~mm}$, and a height of $L=927 \mathrm{~mm}$, giving a radius ratio of $\eta=r_{i} / r_{o}=0.716$ and an aspect ratio of $\Gamma=L /\left(r_{o}-r_{i}\right)=11.7$. The top and bottom caps rotate along with the outer cylinder. The apparatus was filled with water and actively cooled to keep the temperature constant. The torque is measured on the middle section of the inner cylinder using a new co-axial torque transducer (Honeywell 2404-2K, maximum capacity of $225 \mathrm{Nm}$ ), with improved accuracy compared to our former load cell [84]. The azimuthal velocity is obtained by laser Doppler anemometry (LDA), see Fig. 5.1. The laser beams go through the outer cylinder and are focused in the middle of the gap.The water is seeded with $5 \mu \mathrm{m}$ diameter polyamide tracer particles (Dantec) with a maximum Stokes number of St $=\tau_{p} / \eta_{K}=0.004 \ll 1$. Curvature effects of the outer cylinder to our LDA system are accounted for by numerically ray-tracing the LDA-beams [130].

First we follow trajectory I and V shown in the parameter space of Fig. 5.2, which have as a characteristic that $f_{i}-f_{o}=8 \mathrm{~Hz}$ is kept constant (except for the initial and the final part) and is equivalent to approximately $\mathrm{Ta}=10^{12}$ or $\mathrm{Re}=$ $\left(\omega_{i} r_{i}-\omega_{o} r_{o}\right)\left(r_{o}-r_{i}\right) / v=\mathscr{O}\left(10^{6}\right)$. While traversing the trajectory we continuously

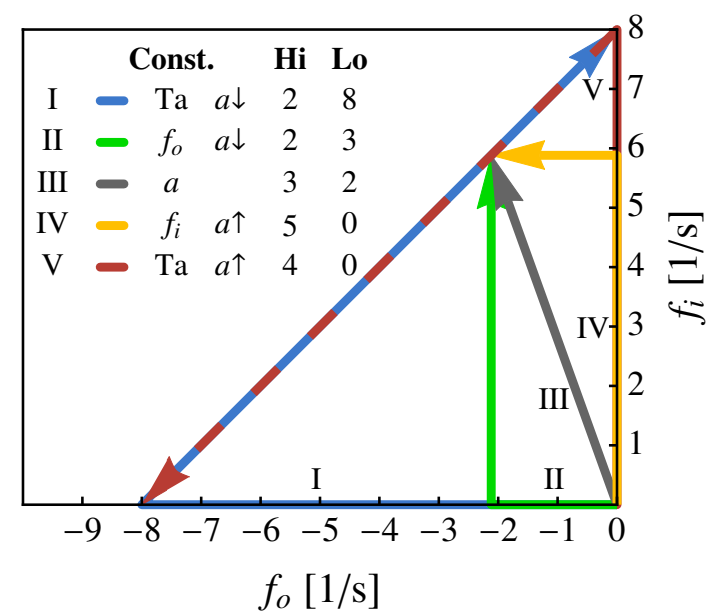

Figure 5.2: Phase space of the trajectories I to V. The arrows indicate the direction in which the phase space is probed. The legend shows which parameter is kept constant, whether the parameter $a=-f_{o} / f_{i}$ is going down $(a \downarrow)$ or up $(a \uparrow)$, and the number of times it went to a high (Hi) or a low (Lo) state. All trajectories go through (or end at) $f_{i}=5.882 \mathrm{~Hz}$, $f_{o}=-2.118 \mathrm{~Hz}\left(a=0.36\right.$, and $\mathrm{Ta} \approx 10^{12}$ or $\left.\operatorname{Re} \approx 10^{6}\right)$. 
measure the torque, scanning over $a$ in one experiment. This is in contrast to experiments that were performed before, where the torque was measured by performing separate ramps of constant $a=-f_{o} / f_{i}[84,85,105,107,127]$. We slowly follow a trajectory in phase space, such as to be in a statistically quasi-steady state the entire time [84]. The temperature variation within the system is $0.04 \mathrm{~K}$, the variation during each measurement is $0.3 \mathrm{~K}$, and the mean temperature for each run is between $19^{\circ} \mathrm{C}$ and $24^{\circ} \mathrm{C}$. The Taylor number depends on viscosity and thus temperature; we therefore remove the main temperature dependence by compensating the Nusselt number with $\mathrm{Ta}^{0.4}$, because $\mathrm{Nu}_{\omega} \propto \mathrm{Ta}^{0.4}$ in the present parameter regime. This approach has been followed before, see e.g. [84, 85].

As can be seen in Fig. 5.3, for increasing $a$ (red shades, trajectory V), the torque is continuous and shows a peak around $a=0.36$, as found before [84, 85, 107, 127]. For trajectory I the torque is found to be the same as trajectory $\mathrm{V}$ for $a<0.17$ and $a>0.51$, however for $0.17<a<0.51$ the torque is found to be different. For decreasing $a$ the system is able to enter another state around $a=0.51$ which is characterised by a lower torque (from here on called 'low state'), around $a=0.17$ the system sharply jumps back to a higher torque state ('high state'), see also the close up view in Fig. 5.4. We have repeated these experiments in order to see how sharp this transition is, and to see in which state the system is, see Fig. 5.2. For trajectory $\mathrm{V}$ we observe that it always goes into the high state, while for the reverse trajectory I the system goes to the low state (for $0.17<a<0.51$ ) with a high probability (8 out of 10$)$.

To verify that the high and low torque states originate from two different physical states, we measure the azimuthal velocity at half-height $z=L / 2$ and center of the gap $r=\left(r_{i}+r_{o}\right) / 2$, while the system is let to move along trajectories I and $\mathrm{V}$ in phase space, see Fig. 5.3b. As in Fig. 5.3a, it is found that the local velocity inside the system bifurcates and that two states are possible. The presence of multiple states in a local measurement (azimuthal velocity) and at the same time a global measurement (torque) provides convincing evidence that the system can indeed be in different turbulent states, despite the very high Taylor number of $\mathscr{O}\left(10^{12}\right)$ (ultimate regime).

To further characterize the turbulent state of the system, we perform axial scans of the azimuthal velocity in the top half of our apparatus for several $a$ for both the high and the low state, see Figs. 5.3c and 5.3d. For each $a$ the azimuthal velocity is standardized (zero mean, unit standard deviation) and color-coded. Fig. 5.3c shows the local velocity for the high state (trajectory V), and shows the presence of 5 large minima/maxima for $a \leq 0.45$; a clear characteristic of 4 turbulent Taylor vortices. For $a \geq 0.5$ the state of the system is less clear, and the system appears to jump between states (without a well-defined $\boldsymbol{a}$-dependence), as seen in the local velocity in Fig. 5.3b. This behavior looks similar to what was found in $\mathrm{RB}$ convection in the classical turbulent regime [116]. However, the mechanism is 


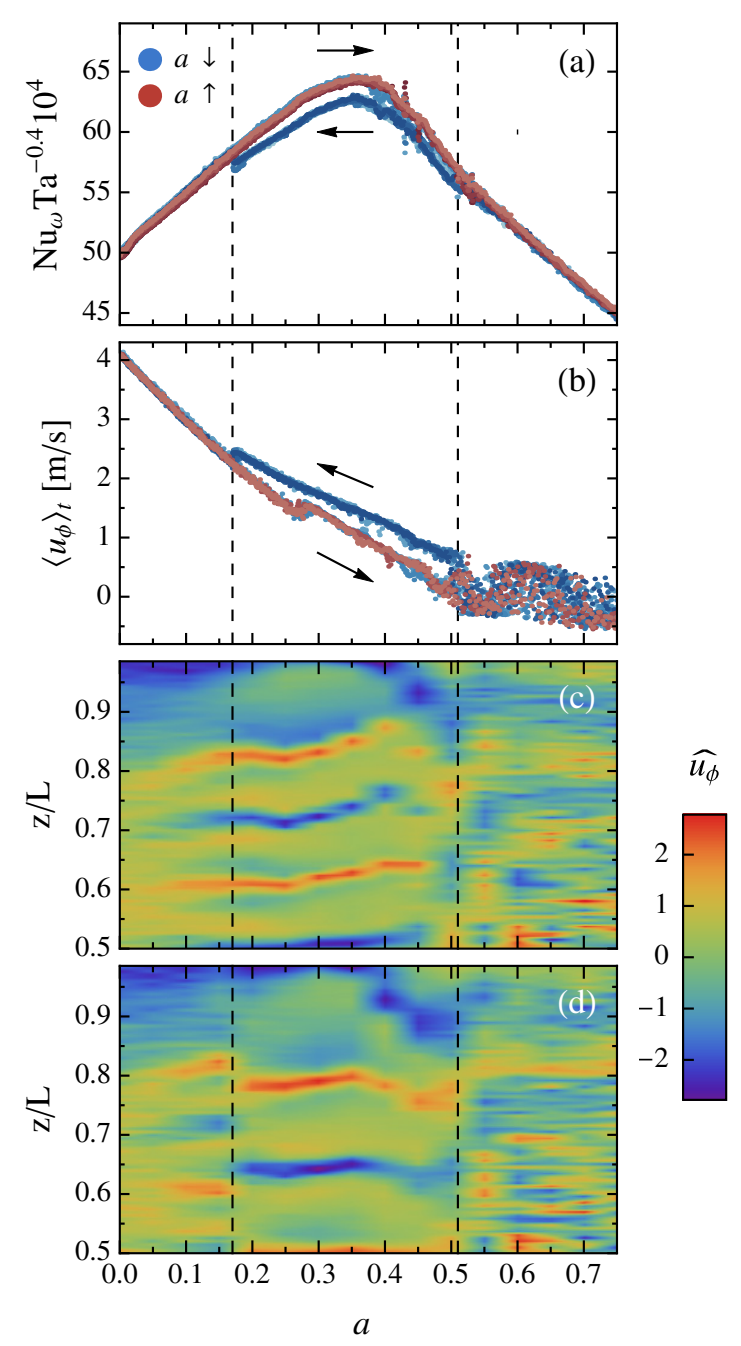

Figure 5.3: (a) Compensated $\mathrm{Nu}_{\omega}$ as a function of $a$. Experiments following trajectories I and $\mathrm{V}$ are colored in blue and red, respectively. Experiments following trajectory I either go into a high or a low state for $0.17<a<0.51$, while experiments of trajectory $\mathrm{V}$ are always in the high state. (b) Azimuthal velocity measured at $r=\left(r_{i}+r_{o}\right) / 2$ and $z / L=0.5$ as a function of $a$ for trajectories I and V. Same colors as in Fig. (a). For the local velocity we also see that the system bifurcates when following trajectory I around $a=0.51$, choosing the high or low state for $0.17<a<0.51$. (c) and (d) show axial scans of the standardized $u_{\phi}$ for varying $a$ following trajectories $\mathrm{V}$ and $\mathrm{I}$, respectively. 4 rolls are present in the top half of the system in Fig. (c), while in Fig. (d) only 3 rolls are present for $0.17<a<0.51$. For $a<0.17$ and $a>0.51$ the system is in the same state, regardless of the trajectory. $\widehat{u_{\phi}}=\left(u_{\phi}-\left\langle u_{\phi}\right\rangle_{z}\right) / \sigma_{a}\left(u_{\phi}\right)$, where $\sigma_{a}$ is the standard deviation of $u_{\phi}$ for each $a$, and \langle\rangle$_{x}$ stands for averaging over $x$. The torque of trajectory V is $2.5 \%$ larger than for trajectory I at $a=0.36$. 


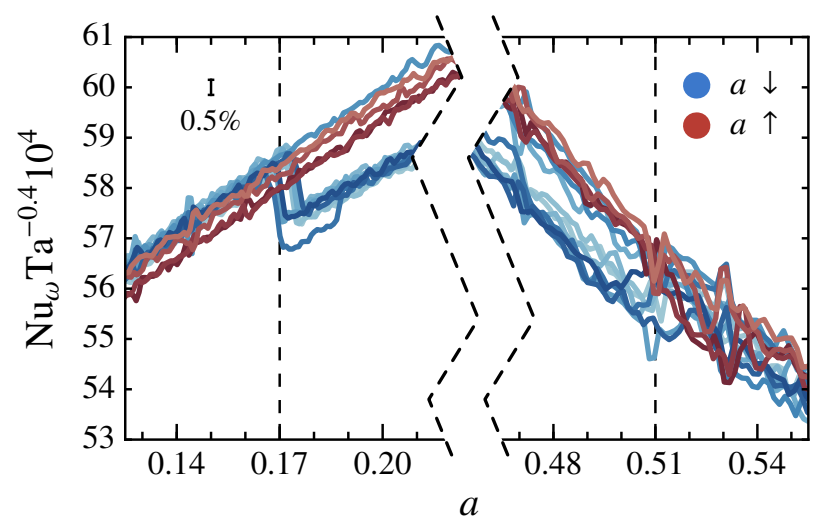

Figure 5.4: Close up view of Fig. 5.3a. The trajectories I $(a \downarrow)$ that are in the low state sharply transition to the high state around $a=0.17$. Trajectories I start to transition around $a=0.51$, though the process seems more gradual, and sometimes stay in the high state. Trajectories V $(a \uparrow)$ never transition and are always in the high state. The error bar, shown in the top-left of the figure, is based on the accuracy of the torque sensor in the system.

different owing to the shorter turnover time scale of the TC system, and the presence of the additional control parameter $a$ in TC, with which we can control the transition. Furthermore, the observed transition is different from Ref. [122] where spontaneous switching back and forth between two states was found, based on global and local measurements. Fig. 5.3d shows the same switching behavior as the high state of Fig. 5.3c for $a$ outside [0.17,0.51]. For $0.17<a<0.51$ it is found that the azimuthal velocity has 4 large minima/maxima, which is the signature of 3 turbulent Taylor vortices (in the top half of the system).

In addition, we provide, for selected $a$, the angular velocity profiles as lines in Fig. 5.5. The angular profiles for trajectories I and V are identical (within experimental and statistical error) for $a$ outside $[0.17,0.51]$. For $a \in[0.17,0.51]$ the profiles are different and show distinguishing features of 3 or 4 rolls $(6$ and 8 rolls in the entire setup), see also the schematics on the right of Fig. 5.5. At the boundary of the last (top) roll and the penultimate roll high velocity fluid from the inner cylinder is advected towards the middle, increasing the velocity at the center of the gap. Similarly, at the boundary of the penultimate and the antepenultimate roll the low velocity fluid from the outer cylinder is advected inwards, decreasing the velocity at the center of the gap. The corresponding average aspect ratio of the vortices is 1.96 ( 3 rolls) and 1.46 ( 4 rolls), which is consistent with previous studies (see e.g. Fig. 2.5 in ref. [131]). Close inspection of Fig. 5.3c and Fig. 5.5a shows that the roll around $z / L=0.5$ slightly drifts upwards for $0.3<a<0.45$ (compared to $a<0.3$ ), which could be a signature of symmetry breaking; the top half of the system behaves slightly differently from the bottom half. This is an explana- 

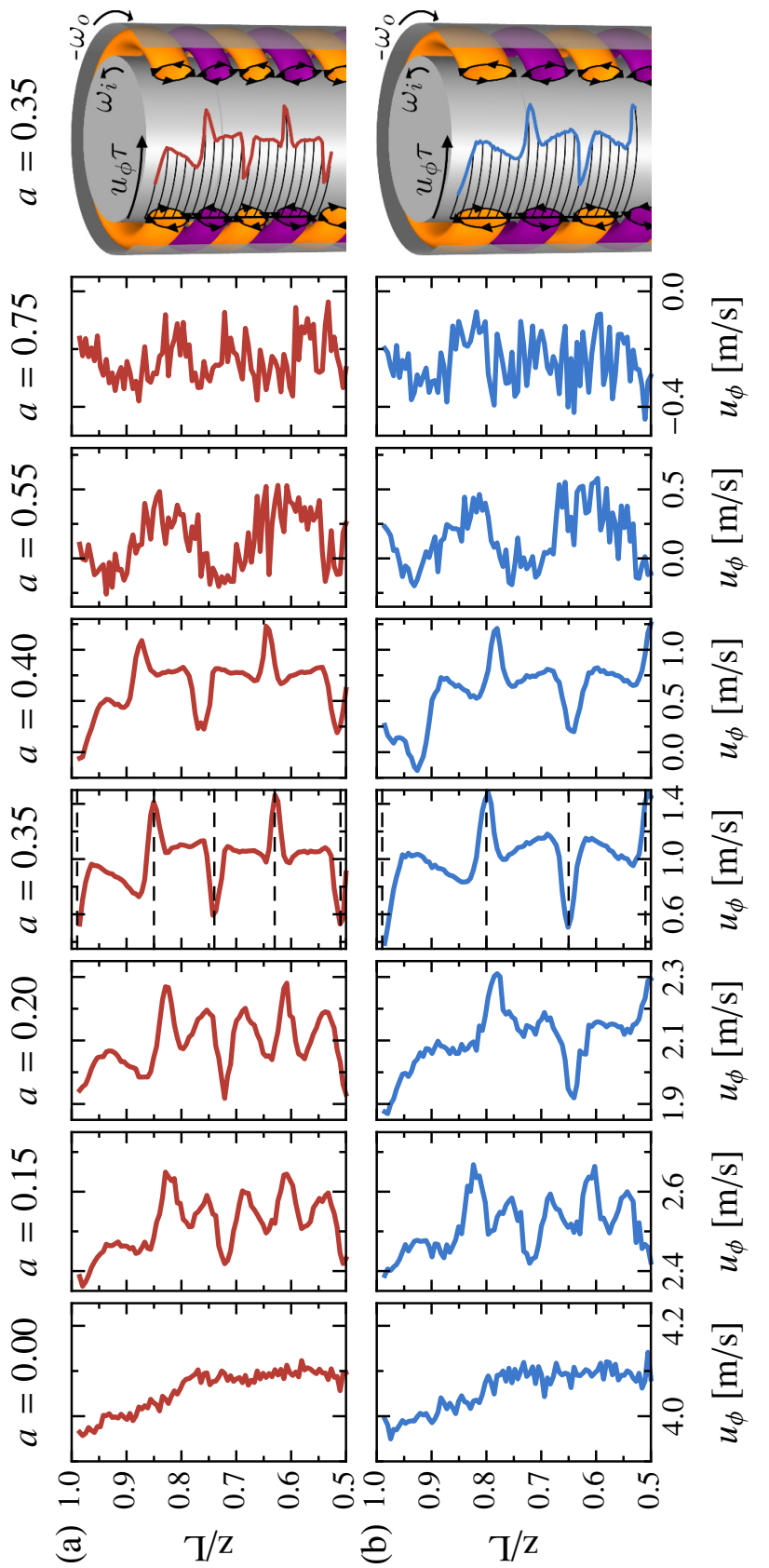

e $\mathrm{T} / \mathrm{z}$

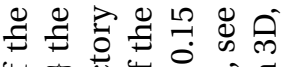

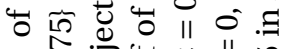

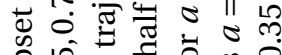

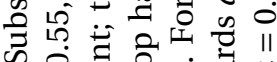

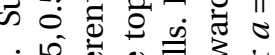
ن

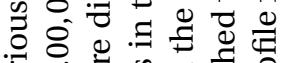
范

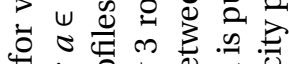

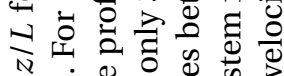

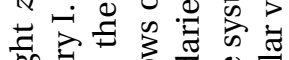

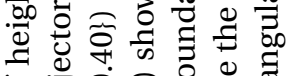
पँ.

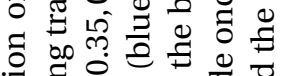

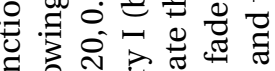
Ð สิ

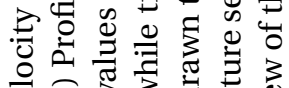

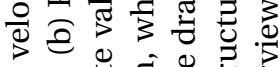

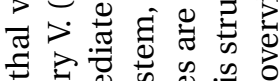

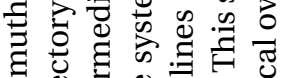
娄 bo 10 क ज्ञ .ำ Tु

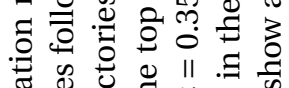

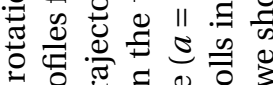

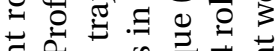

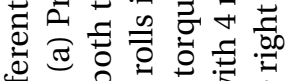

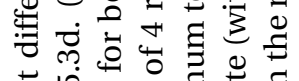
ส

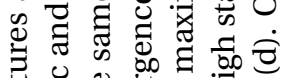
冚

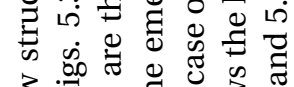
3.

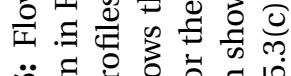
เอ

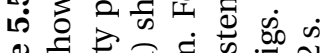

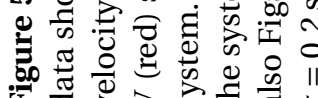


tion of the anomaly in the local angular velocity for trajectory $\mathrm{V}$ around $a \approx 0.28$. Such symmetry-breaking behavior has also been found in von Kármán flow [132]. Note that the middle section of the inner cylinder with height $z / L=0.578$ does not cover all the rolls, therefore the exact mean torque value over the entire inner cylinder could be different. It is, however, unlikely that this difference takes away the 'jumping' behavior.

From our findings of trajectory I and $\mathrm{V}$ (constant Ta) we find that the maximum torque is at $a=0.36\left(f_{i}=5.882 \mathrm{~Hz}, f_{o}=-2.118 \mathrm{~Hz}\right)$. We now look at other trajectories reaching this maximum but keeping either $f_{o}, a$, or $f_{i}$ constant, see trajectories II-IV in Fig. 5.2. For constant $f_{o}$ and constant $a$ (trajectories II and III) the system was found to have the ability to go in either the high or the low state as well, see the legend of Fig. 5.2. Like trajectory V, trajectory IV (constant $f_{i}$ ) was found to be characterised by a high state of the system. It seems that if $a=0.36$ is approached from below (trajectories IV and V) the flow does not bifurcate. However, the system does bifurcate when $a=0.36$ is approached from the top, or if $a$ is kept constant but the driving strength is increased.

\subsection{Discussion}

We have shown that Taylor-Couette flow displays flow structure even for a very high Taylor number of roughly $10^{12}\left(\operatorname{Re}=\mathscr{O}\left(10^{6}\right)\right)$, which is beyond Reynolds numbers for which large-scale structures were believed to vanish in Kolmogorov's picture. In addition, we found that the system is hysteretic and can be in multiple stable turbulent states for the same driving parameters. The multiple states are simultaneously measured globally and locally by performing torque and LDA measurements. It was found that multiple states can occur for rotation ratios $0.17<a<0.51$. For $0 \leq a<0.17$ there is only a single stable state, though we cannot exclude that other trajectories in phase space might trigger multiple states in this region. For $a>0.51$ the system does not possess a state with a clear roll structure. Presently, a theoretical understanding of the values $a=0.17, a=0.51$ and the sharp and smooth behaviour of the jumps around those $a$ is lacking. Finally we note that the presented experiments, performed in the $\mathrm{T}^{3} \mathrm{C}$ facility, will be challenging to simulate in DNS in the foreseen future: simulations of such high Ta are difficult, especially for $\Gamma=\mathscr{O}(10)$. The present work highlights the importance of the coherent structures and their selectability in highly turbulent flows, which demand continued effort and investigation. The question of whether or not these structures survive for even larger Reynolds numbers, is important for understanding the myriad of large-scale flows in nature, and will be treated in chapter 6 . 


\section{6}

\section{Exploring the phase space of multiple states in highly turbulent Taylor-Couette flow*}

We investigate the existence of multiple turbulent states in highly turbulent TaylorCouette flow in the range of $\mathrm{Ta}=10^{11}$ to $9 \cdot 10^{12}$, by measuring the global torques and the local velocities while probing the phase space spanned by the rotation rates of the inner and outer cylinder. The multiple states are found to be very robust and are expected to persist beyond $\mathrm{Ta}=10^{13}$. The rotation ratio is the parameter that most strongly controls the transitions between the flow states, and the transitional values only weakly depend on the Taylor number. However, complex paths in the phase space are necessary to unlock the full region of multiple states. Lastly, by mapping the flow structures for various rotation ratios in a Taylor-Couette setup with an equal radius ratio but a larger aspect ratio than before, multiple states were again observed. Here, they are characterized by even richer roll structure phenomena, including, for the first time observed in highly turbulent TC flow, an antisymmetrical roll state.

${ }^{*}$ Submitted as: Roeland C. A. van der Veen, Sander G. Huisman, On-Yu Dung, Ho L. Tang, Chao Sun and Detlef Lohse, Exploring the phase space of multiple states in highly turbulent Taylor-Couette flow. Experiments by RVDV, SH, OD and HLT, writing by RVDV, supervision by CS and DL. 


\subsection{Introduction}

Taylor-Couette (TC) flow, the flow between two coaxial cylinders that can independently rotate, is one of the paradigmatic systems of fluid physics. In the past century, a large range of phenomena and concepts have been studied in this system, such as instabilities, nonlinear dynamics and spatiotemporal chaos, pattern formation and turbulence. It has been used extensively as a model system in fluid dynamics because it is a closed system, has a relatively simple geometry and therefore has multiple symmetries. This also means that it is experimentally accessible with high precision. For a historical overview of Taylor-Couette research, the reader is referred to Donnely et al. [133], while the reviews of Di Prima et al. [60] and Fardin et al. [104] treat the rich flow structures of TC flow at the onset of instabilities and slightly above. The state-of-the-art of high Reynolds number TaylorCouette turbulence is treated by Grossmann, Lohse, and Sun [11], which is the regime we focus on in the present work. Since the research of Wendt [134] in 1933 and the Austin-Maryland experiments $[83,105,135]$ in the nineties, only in the past decade more researchers have started to explore the strongly turbulent regime with independently rotating cylinders, both experimentally $[75,85,107,126-128$, 136-138] and numerically [109, 123, 129, 139-142].

The geometrical parameters of the TC system are the inner and outer cylinder radii $r_{i}$ and $r_{o}$ respectively, the gap width $d=r_{o}-r_{i}$, and the height of the setup $L$. These can be expressed in dimensionless form by the radius ratio $\eta=r_{i} / r_{o}$ and the aspect ratio $\Gamma=L / d$. The inner and outer cylinder rotate with angular velocities $\omega_{i, o}=2 \pi f_{i, o}$, which can be expressed in dimensionless form by the Reynolds numbers $\operatorname{Re}_{i, o}=\omega_{i, o} r_{i, o} d / v$ where $v$ is the kinematic viscosity. Using the analogy of TC flow with Rayleigh-Bénard (RB) convection [14], the driving of the flow can alternatively be characterized by the Taylor number [12]

$$
\mathrm{Ta}=\frac{(1+\eta)^{4}}{64 \eta^{2}} \frac{\left(r_{o}-r_{i}\right)^{2}\left(r_{i}+r_{o}\right)^{2}\left(\omega_{i}-\omega_{o}\right)^{2}}{v^{2}},
$$

combined with the (negative) rotation ratio

$$
a=-\frac{\omega_{o}}{\omega_{i}}
$$

with $a>0$ for counter-rotation and $a<0$ for corotation. The response of the system is the torque $\tau$ required to sustain constant angular velocity or a 'Nusselt' number [12], $\mathrm{Nu}_{\omega}=\tau / \tau_{\text {laminar }}$, which is the angular velocity flux nondimensionalised with the flux of the laminar, nonvortical, flow.

As we increase the driving strength, we can identify three regimes in TaylorCouette flow. At Taylor numbers that are still low $\left(\mathrm{Ta} \sim 10^{6}\right)$, the gap between the cylinders is filled with coherent structures called Taylor rolls or Taylor vortices 
$[10,104,143]$. When increasing the driving strength (i.e. the Taylor number), turbulence starts to develop in the bulk and boundary layers start to develop. These BLs are still of laminar type, and in analogy [14] with Rayleigh-Bénard flow, we call this regime the classical regime of TC turbulence. In the turbulent bulk, depending on the rotation ratio $a$, either the Taylor rolls survive, they partly survive close to the inner cylinder, or the bulk is featureless [88, 109, 144, 145]. By further increasing $\mathrm{Ta}$, around $\mathrm{Ta} \approx 3 \cdot 10^{8}$, the ultimate regime $[118,125,146]$ is reached, in which also the boundary layers are turbulent. It has to be noted that in the transition to the ultimate regime the bulk characteristics do not change significantly, so in principle the Taylor rolls can survive.

The question, however, is what happens to the roll structures for even stronger driving, e.g. Ta $\gg 10^{10}$ or Re $\gg 10^{5}$. Visualizations by Lathrop et al. [105] show that for pure inner cylinder rotation $(a=0)$, no clear Taylor vortices are visible for Reynolds numbers beyond $\mathrm{Re}=1.2 \cdot 10^{5}$. Also for inner cylinder rotation only, Lewis et al. [83] found that vortex-like structures persist for Reynolds numbers up to $\operatorname{Re}=10^{6}$. However, for Re $>10^{5}$ the vortex boundaries drifted axially and the number of vortices was not well-defined. In chapter 5 we explored the existence of rolls at high Reynolds number for counter-rotating cylinders, and found that stable turbulent roll structures persist at a range of rotation ratios $a>0$ (but not $a=0)$ for very large Taylor numbers of $\mathrm{Ta}=10^{12}\left(\mathrm{Re}=10^{6}\right)$. At pure inner cylinder rotation, no roll structures in the time-averaged azimuthal velocity were found, consistent with the previous findings. Strikingly, the range of rotation ratios at which the structures exist, corresponds to the $a$-range close to optimal momentum transport, reflecting that the optimal transport is connected to the existence of the stable large-scale coherent structures: the Taylor rolls supply the optimal angular velocity transfer from the inner to the outer cylinder.

The main finding of chapter 5 was however, that at these very high Taylor numbers, multiple states corresponding to different roll structures can exist for the same rotation parameters. These observed multiple states are stable, i.e. there is no spontaneous switching and there are no slow transitions between the states. The existence of these multiple turbulent states was unexpected and questioned Kolmogorov's paradigm that suggests that for strongly turbulent flows with their many degrees of freedom and large fluctuations, only one turbulent state would be possible [112, 113].

Multiple states have been observed in other geometries, but the nature of the states is generally different. In Rayleigh-Bénard convection at low Rayleigh number, continuous switching between two different roll states with different heat transfer properties was found [114-116]. At larger Rayleigh numbers no multiple states were observed in a single setup; only when the boundary conditions were changed one could trigger transitions $[117,118]$. However, in rotating RayleighBénard convection for very high Rayleigh numbers up to $2 \cdot 10^{12}$, a sequence of 
sharp transitions was found as the rotation rate was increased [147]. For von Kármán flow multiple turbulent states were found when driving it with impellors with curved blades [119-121]. These studies revealed the spontaneous symmetrybreaking and turbulent bifurcations in highly turbulent von Kármán flow up to $\operatorname{Re}=10^{6}$. Lastly, in spherical-Couette flow spontaneous switching between two turbulent states at fixed rotation rates was observed [122].

In Taylor-Couette flow, multiple states were only observed in the classical regime (see e.g. [10]) before Huisman et al. [148] showed their existence in the ultimate regime. More recently, multiple states in TC flow have again been observed, in the transition to the ultimate regime [145] and beyond [149]. These studies again confirmed that, in the Taylor-Couette geometry, the multiple states manifest themselves as states with a different number of turbulent rolls, which can create a different torque on the cylinders. This leads us to the main implication of the existence of multiple states, which is both fundamental and practical: when extrapolating the known scaling laws for e.g. the angular momentum transport, it is essential to be aware of possible transitions between different turbulent states, which in general possess different values for the torque and the angular momentum transport.

In their review of high Reynolds number TC flow, Grossmann, Lohse and Sun [11] posed questions to be solved by the scientific community. Two of them were: "When further increasing Ta to $\mathrm{Ta} \gg 10^{13}$, will the large-scale coherent structures (turbulent Taylor rolls) continue to exist? If so, will multiple turbulent states still coexist, or will the fluctuation be so large that the turbulent dynamics meanders between these states?" These are two questions that we here can begin to answer, by increasing the maximum Taylor number by almost one order of magnitude to $9 \cdot 10^{12}$ and mapping the (Ta, $a$ ) phase space of multiple states.

Additionally, as mentioned in chapter 5, understanding of the values of $a$ at which the system transitions between states is still lacking. By exploring the phase spanned by the inner and outer cylinder velocities we will investigate how these transitional $a$ values depend on the driving strength, and what parameter most strongly controls the transitions.

The final question we aim to answer in this chapter concerns the influence of the aspect ratio of the system. The multiple states manifest themselves as different roll structures and the aspect ratio of the Taylor-Couette system directly influences the number of rolls it can support. In this chapter we will measure the exact roll structure dependence on the rotation ratio $a$ in a setup with aspect ratio $\Gamma=18.3$, in order to compare the results to previous measurements and to explore the influence of the aspect ratio on the manifestation of multiple states.

We can summarize these questions into one: "How robust are multiple states?". 


\subsection{Setups and methods}

The experiments were performed in the Twente Turbulent TC facility $\left(\mathrm{T}^{3} \mathrm{C}\right)$ [103] and the new Boiling Twente Taylor-Couette facility (BTTC); see Fig. 6.1 for a schematic overview. In the larger $\mathrm{T}^{3} \mathrm{C}$ setup we are able to reach Taylor numbers of up to $\mathrm{Ta}=9 \cdot 10^{12}$ and accurately measure the torque while traversing the phase space of the inner and outer cylinder velocities, in order to map the phase space of multiple turbulent states. The BTTC is designed to study multiphase turbulent flows, but here we will employ the excellent optical access to perform extensive local velocity measurements using laser Doppler anemometry. The systems have equal radius ratio $\eta=r_{i} / r_{o}$ but different aspect ratio $\Gamma=L / d$, allowing us to characterize the importance of the aspect ratio with respect to the behavior of the roll structures, by comparing measurements to those in chapter 5 . We will first discuss the experimental details of the torque and LDA measurements that are performed in the $\mathrm{T}^{3} \mathrm{C}$ facility, after which we will treat the LDA measurements in the BTTC setup. Presently, accurate torque measurements are not possible in the BTTC setup.

(a)

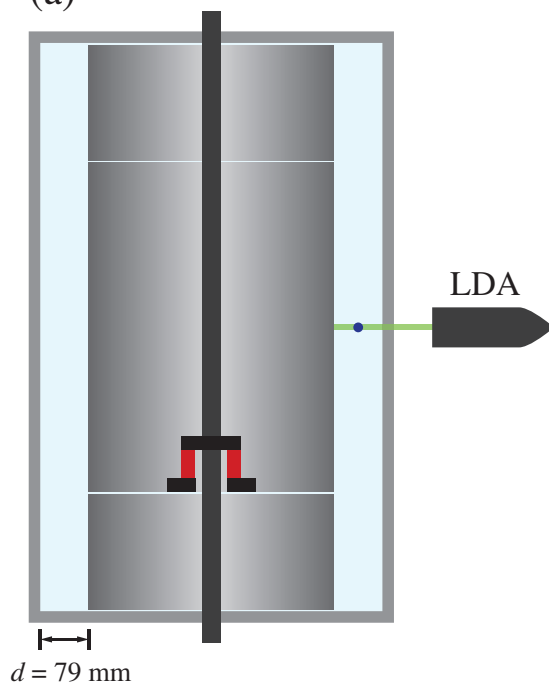

(b)

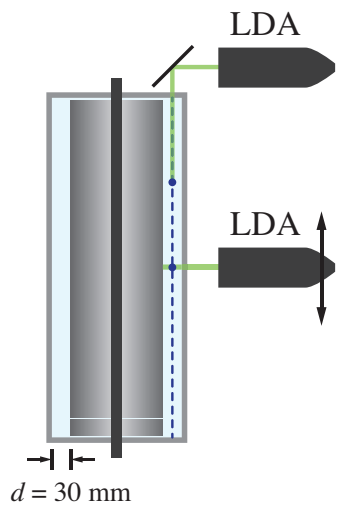

Figure 6.1: Schematic of the cross section of (a) the $\mathrm{T}^{3} \mathrm{C}$ [103] and (b) the BTTC (see chapter 4) setup. (a) The $\mathrm{T}^{3} \mathrm{C}$ apparatus is outfitted with a co-axial torque transducer to measure the torque that the fluid exerts on the inner cylinder. The azimuthal velocity is probed at the middle of the gap and the middle of the height of the apparatus using laser Doppler anemometry (LDA). (b) In the BTTC apparatus the azimuthal and radial velocity at the middle of the gap and 0.75 of the height are measured through the transparent top plate using a $45^{\circ}$ mirror. Additionally, a full height scan of the azimuthal velocity is made by traversing the LDA head vertically. 
The $\mathrm{T}^{3} \mathrm{C}$ apparatus has an inner cylinder with radius $r_{i}=200 \mathrm{~mm}$ and a transparent outer cylinder with inside radius $r_{o}=279.4 \mathrm{~mm}$ and a height of $L=927 \mathrm{~mm}$. This gives a radius ratio of $\eta=r_{i} / r_{o}=0.716$ and an aspect ratio of $\Gamma=L /\left(r_{o}-r_{i}\right)=11.7$. The maximum rotation rates of the inner and outer cylinder are $20 \mathrm{~Hz}$ and $10 \mathrm{~Hz}$, respectively, giving maximum inner and outer Reynolds numbers of $\operatorname{Re}_{i}=2.0 \cdot 10^{6}$ and $\mathrm{Re}_{o}=1.4 \cdot 10^{6}$ with water at room temperature as working fluid. The top and bottom caps rotate along with the outer cylinder. The apparatus was filled with water and actively cooled at the top and bottom plate to keep the temperature constant. Due to excellent turbulent mixing of the fluid, the spatial temperature variation within the system is less than $0.1 \mathrm{~K}$.

In chapter 5 it was found that changes between different roll states manifest themselves as 'jumps' in the torque that acts on the cylinders. The torque is measured on the middle section of the inner cylinder using a co-axial torque transducer (Honeywell 2404-2K, maximum capacity of $225 \mathrm{Nm}$ ). The middle section of the inner cylinder with height $z / L=0.578$ does not cover all the rolls, therefore the exact mean torque value over the entire inner cylinder could be different. This, however, does not take away the jumps observed in the torque when the flow undergoes transitions between different roll structures.

The azimuthal velocity is obtained by laser Doppler anemometry (LDA), see Fig. 6.1(a). The laser beams go through the outer cylinder and are focused in the middle of the gap. Curvature effects of the outer cylinder on our LDA system are accounted for by numerically ray-tracing the LDA-beams [130]. The water is seeded with $5 \mu \mathrm{m}$ diameter polyamide tracer particles (Dantec) with a maximum Stokes number of $S t=\tau_{p} / \tau_{K}=0.017 \ll 1$ (with $\tau_{p}$ the particle response time and $\tau_{K}$ the Kolmogorov timescale), so that they can be considered to be perfect tracers of the flow.

To answer the questions we posed in the introduction, we measured the torque on the cylinders while quasistatically traversing the phase space along different types of trajectories shown in Fig. 6.2. Specific trajectories such as those of type III were used to find the extent of the phase space in which multiple states are possible, as will be shown in the results section.

We slowly followed the trajectories in phase space by changing the cylinder velocities with $4 \mathrm{~Hz} / \mathrm{h}$, found to be slow enough to make acceleration/deceleration effects negligible and give the system ample time to stabilize into any flow state [84]. The beginnings of the trajectories were reached by spinning the cylinders up to the initial velocities at constant $a$ with $4 \mathrm{~Hz} / \mathrm{min}$, after which the system was kept at these velocities for five minutes to remove any transitional effects. The mean water temperature for each run was between $20^{\circ} \mathrm{C}$ to $27^{\circ} \mathrm{C}$, while the temperature variation during each measurement was smaller than $0.5 \mathrm{~K}$. The Taylor number depends on viscosity and thus on temperature; we therefore removed the main temperature dependence by compensating the Nusselt number with $\mathrm{Ta}^{0.4}$, 


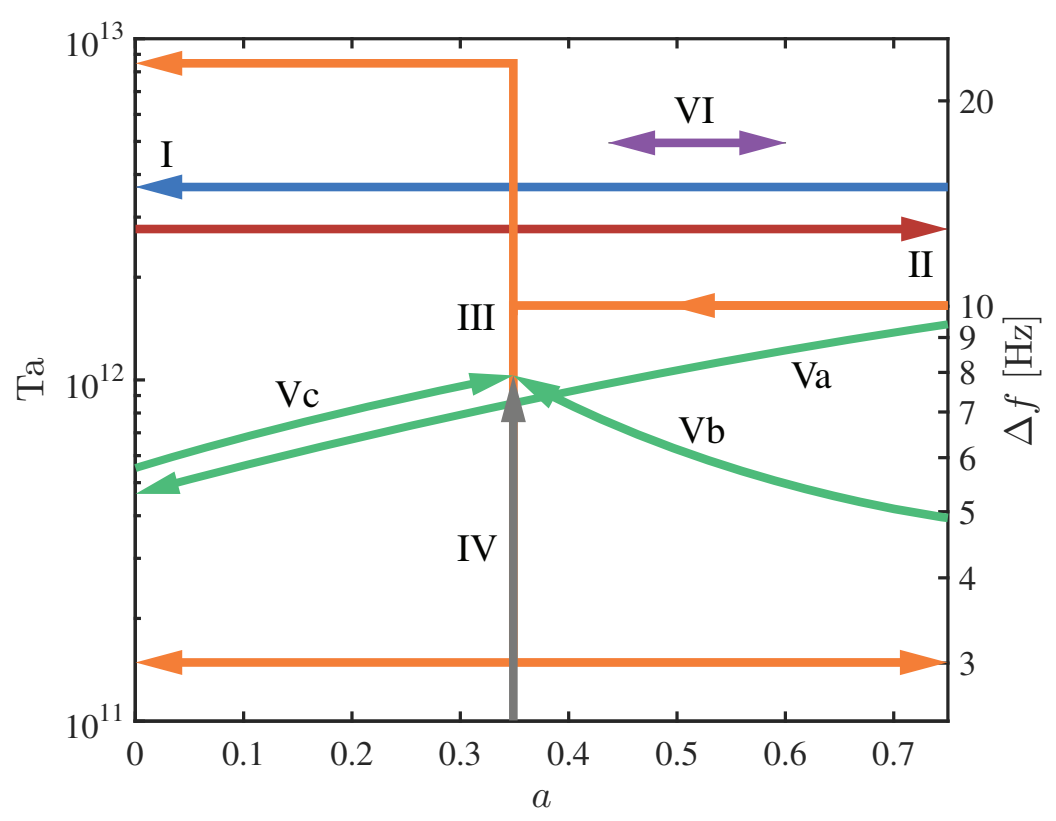

Figure 6.2: Representative examples of the types of measurement trajectories in the phase space (Ta, $a$ ): constant Ta with decreasing $a=-f_{o} / f_{i}$ (I) and increasing $a$ (II), constant Ta with a change to different Ta in between (III), increasing Ta at constant $a$ (IV), fixed $f_{i}$ with decreasing $\left|f_{o}\right|(\mathrm{Va})$, fixed $f_{o}$ with increasing $\left|f_{i}\right|(\mathrm{Vb})$, fixed $f_{i}$ with increasing $\left|f_{o}\right|(\mathrm{Vc})$ and constant Ta back and forth (VI). For the right axis a temperature of $22^{\circ} \mathrm{C}$ is used to convert from the Taylor number to $\Delta f=f_{i}-f_{o}$.

because $\mathrm{Nu}_{\omega} \propto \mathrm{Ta}^{0.4}$ in the present parameter regime. This approach has been followed before in chapter 5 and Refs. [84, 85].

We now turn our attention to the BTTC facility (see chapter 4 for more details). This setup has an inner cylinder with radius $r_{i}=75 \mathrm{~mm}$ and a transparent outer cylinder with inside radius $r_{o}=105 \mathrm{~mm}$, and a height of $L=549 \mathrm{~mm}$. This gives a radius ratio of $\eta=r_{i} / r_{o}=0.714$, very close to that of the $\mathrm{T}^{3} \mathrm{C}$ setup, and an aspect ratio of $\Gamma=L /\left(r_{o}-r_{i}\right)=18.3$, considerably larger than that of the $\mathrm{T}^{3} \mathrm{C}$ setup. The maximum rotation rates of the inner and outer cylinder are again $20 \mathrm{~Hz}$ and $10 \mathrm{~Hz}$, respectively, giving maximum inner and outer Reynolds numbers of $\operatorname{Re}_{i}=2.8 \cdot 10^{5}$ and $\operatorname{Re}_{o}=2.0 \cdot 10^{5}$ with water at room temperature as the working fluid. The top and bottom caps rotate along with the outer cylinder. The top plate and outer cylinder are fully transparent, enabling excellent optical access for optical measurement techniques such as laser Doppler anemometry. The apparatus was filled with water and actively cooled at the inner cylinder to keep the temperature constant. The spatial temperature variation within the system is less than $0.1 \mathrm{~K}$. 
Two types of LDA measurements were performed in the BTTC setup. The first consisted of measuring the local velocity at a single point continuously while slowly changing $a$, in order to see transitions between the multiple states. With the second set of experiments we characterized the roll structures by performing finely spaced measurements of the velocity over the height of the setup, for several values of $a$. For each type of measurement, a different way of mounting the LDA system was employed, as shown in Fig. 6.1(b). Using a mirror mounted at an angle of $45^{\circ}$, we could measure the azimuthal and radial velocity in the flow. Although measuring the azimuthal velocity is a good way of characterizing rolls, as we have seen in chapter 5 , measuring the radial velocity gives a more direct quantification of the rolls. We quasistatically traversed the phase space by either decreasing or increasing $a$ while keeping the difference in cylinder velocities constant (and so Ta), while simultaneously measuring the two velocity components with LDA at the middle of the gap and at $z / L=0.75$, showing transitions between different roll states. This was done at $\Delta f=f_{i}-f_{o}=10 \mathrm{~Hz}$, giving a Taylor number of $\mathrm{Ta}=3 \cdot 10^{10}$. Again $5 \mu \mathrm{m}$ diameter polyamide tracer particles were used, which here have a maximum Stokes number of St $=\tau_{p} / \tau_{\eta}=0.002 \ll 1$.

In addition to these continuous measurements of the velocity, we performed axial scans using LDA. The LDA head was positioned on a vertical translation stage with the focus at the middle of the gap (optical effects were again accounted for using a ray-tracer). The measurements were performed in a similar manner as in chapter 5, and can be summarized as follows: we spun the cylinders up to either $a=0$ or $a=0.75$, acquired velocity data at many points along the height of the cylinder, slowly changed the velocity to the next value of $a$, repeated the velocity acquisition, again slowly changed to the next $a$, and so forth. At every height the velocity was measured for $20 \mathrm{~s}$, giving approximately 10000 data points each. The axial step size was $1.5 \mathrm{~mm}$ and the step size in $a$ was 0.05 , refined to 0.025 around $a=0.4$. The measurement height was not traversed monotonically, but divided in groups of heights, which were measured non-consecutively. We did so in order to detect possible slow transitions of the flow, which then would show as discontinuities in the resulting velocity profiles. 


\subsection{Phase space of multiple states in the $\mathbf{T}^{3} \mathbf{C}$ with $\Gamma=11.7$}

As observed before in chapter 5 , different flow states can be triggered by either decreasing (trajectory type I) or increasing (trajectory type II) the rotation ratio $a$ at constant driving parameter Ta. In this chapter we perfomed these measurements at several different values of the Taylor number between $\mathrm{Ta}=1.4 \cdot 10^{11}$ and $\mathrm{Ta}=8.8 \cdot 10^{12}$, of which some examples are shown in Fig. 6.3(a). For the case of increasing $a$, the torque is continuous and shows the familiar peak around $a=0.36$ $[84,85,88,107,127]$. But when $a$ is decreased, the system can enter a state with
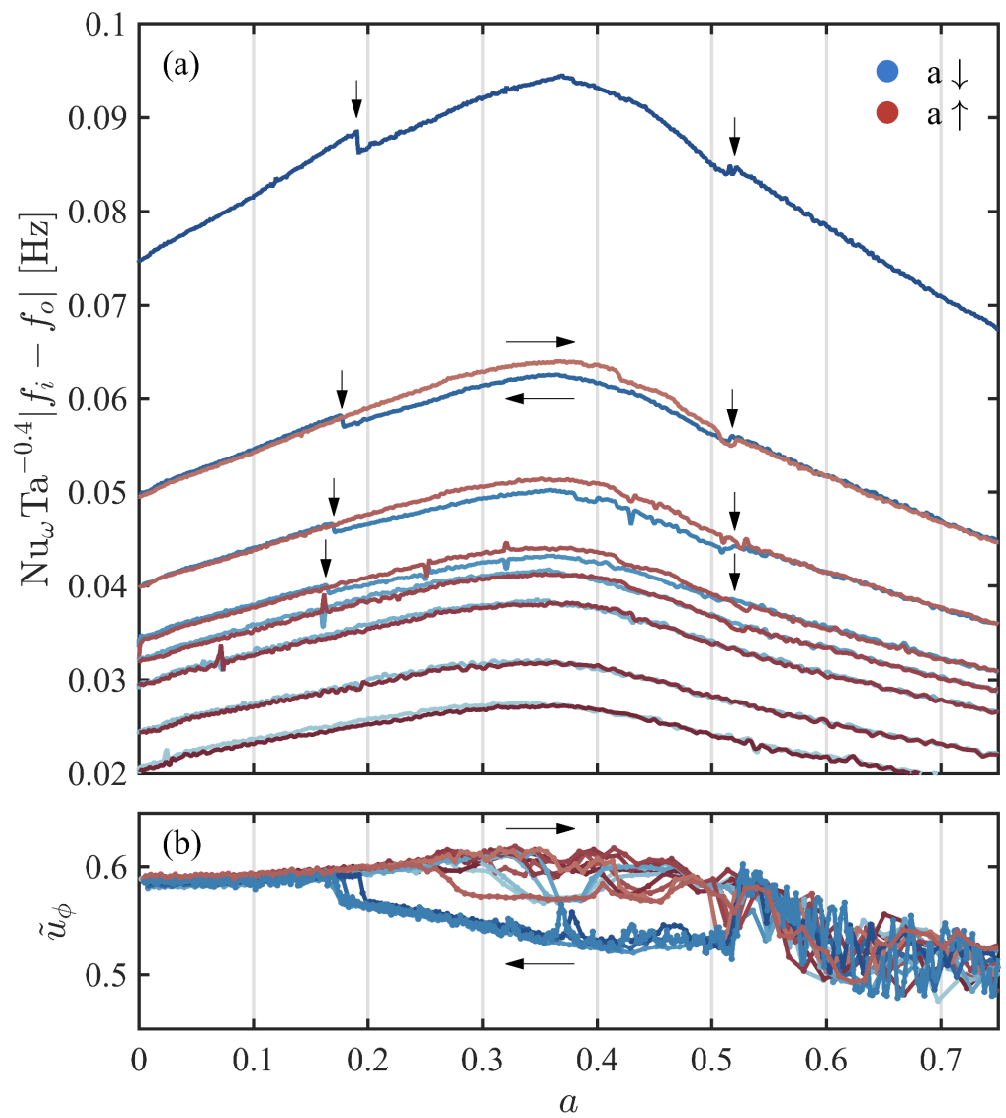

Figure 6.3: Global torque and local velocity versus rotation ratio for several values of Ta, corresponding to trajectories of type I (blue) and II (red) in Fig. 6.2. (a) Compensated $\mathrm{Nu}_{\omega}$ as a function of $a$, multiplied by $\left|f_{i}-f_{o}\right|$ to shift the lines for clarity, for $\Delta f=4.3 \mathrm{~Hz}, 5 \mathrm{~Hz}$, $6 \mathrm{~Hz}, 6.5 \mathrm{~Hz}, 7 \mathrm{~Hz}, 8 \mathrm{~Hz}, 10 \mathrm{~Hz}$ and $15 \mathrm{~Hz}$. The vertical arrows denote the transitions to the low and high torque states. (b) Azimuthal velocity $\tilde{u}_{\phi}=\left(\left\langle u_{\phi}\right\rangle_{t}-u_{i}\right) /\left(u_{o}-u_{i}\right)$ measured at $r=\left(r_{i}+r_{o}\right) / 2$ and $z / L=0.5$ as a function of $a$. 
a lower torque (called 'low state') at a certain value $a>0.36$, jumping back to a higher torque state ('high state') at a certain value $a<0.36$. This bifurcation is confirmed by measuring the local velocity using laser Doppler anemometry, see Fig. 6.3(b). The transition to the low state is a probabilistic phenomenon, although the probability is high (8 out of 10 for trajectories at $\Delta f=8 \mathrm{~Hz}$ ).

By performing several more measurements with finely spaced Taylor number it was found that for the type I and II trajectories of constant Ta, multiple states only appear for Ta $>(7.7 \pm 0.2) \cdot 10^{11}$. We stress that this does not imply anything about the existence or strength of roll structures, but rather the ability of the system to switch between the different roll structures. Of the two possible roll states, the 8-roll state corresponding to the high torque state seems to be more favorable than the 6-roll state corresponding to the low torque state (see chapter 5).

From these measurements we can draw two intermediate conclusions, on which we will then further build. The first is that multiple states still occur for higher Ta than observed before, but also only beyond a certain high enough threshold Taylor number. The second is that there seems to be a (weak) dependence of the transitional $a$-values on the Taylor number.

Refer now to Fig. 6.4, as we make a journey through the trajectories and fill the phase space with the transitions between the low and high torque state. We have already seen that at constant Ta and increasing $a$ no transitions are present, but for decreasing $a$ we can plot the blue symbols in the figure. As mentioned, below $\mathrm{Ta}=(7.7 \pm 0.2) \cdot 10^{11}$ the low state cannot be reached using these trajectories. We find that by following a type IV trajectory of constant $a$ and increasing $\mathrm{Ta}$, the system sometimes is in the low state, however with a low probability (see chapter 5). To further explore the boundaries of the multiple states, we follow the recipe of the type III trajectories, by starting at $a=1$ and $\Delta f=10 \mathrm{~Hz}$, then reducing $a$ at constant Ta to relatively reliably enter the low state. Then Ta is reduced at constant $a$, crossing the line of $\mathrm{Ta}=7.7 \cdot 10^{11}$, after which $a$ is either decreased or increased. The system stays in the low state until jumping back to the high state at $a=0.15$ to 0.17 and $a=0.53$ to 0.55 , respectively. This greatly extends the knowledge of the boundaries of the region where multiple states are possible. To gather more points at the left boundary of the phase space, the Taylor number is also increased to $\mathrm{Ta}=8.8 \cdot 10^{12}$, corresponding to a combined Reynolds number of $\operatorname{Re}=\left(\omega_{i} r_{i}-\omega_{o} r_{o}\right) d / v=2.6 \cdot 10^{6}$. Concluding, multiple states are observed for almost two orders of magnitude in the Taylor number within a well-defined range of rotation ratios.

We wonder whether the transitional $a$ depends on the way one traverses the boundary of the multiple states. For this, we perform experiments at constant $f_{i}$ or $f_{o}$, traversing the (Ta, $a$ ) phase space diagonally. Along trajectories of type Va the system undergoes transitions at very similar $a$ as compared to the other trajectories, confirming the idea that the rotation ratio is the parameter that controls 


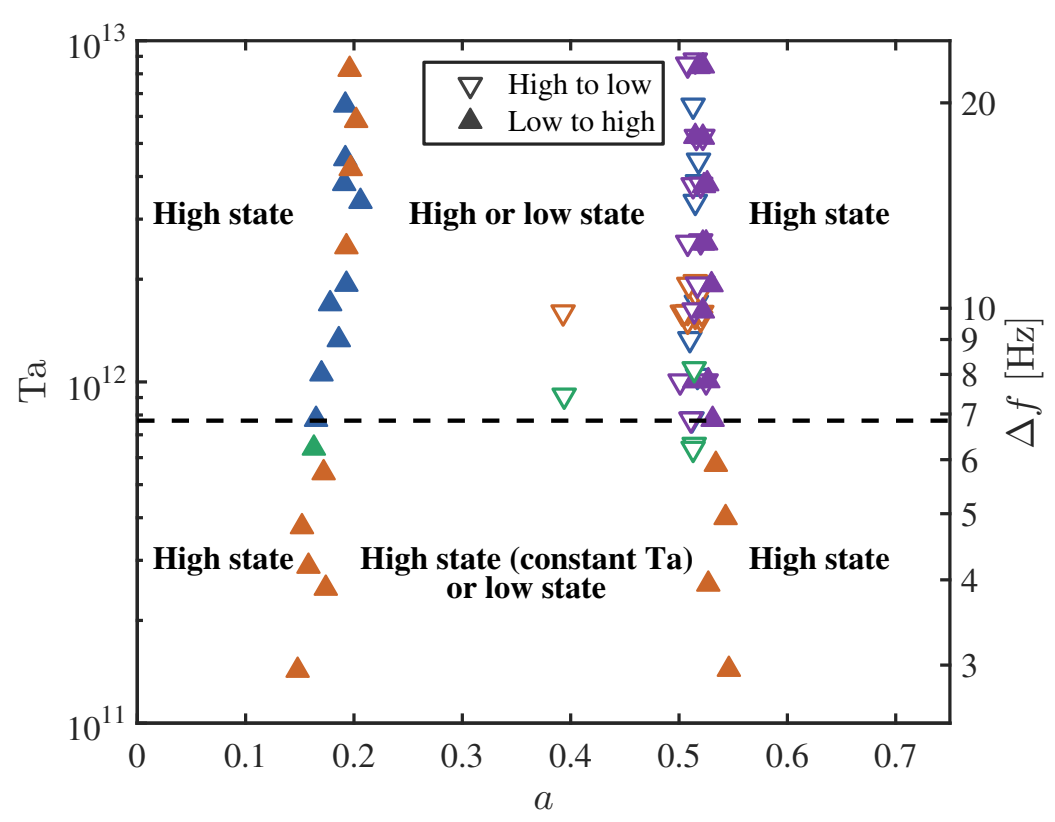

Figure 6.4: The phase space (Ta,a) showing transitions from the high to low state by open symbols and transitions from the low to high state by closed symbols. The dashed line is at $\mathrm{Ta}=7.7 \cdot 10^{11}$, below which the system does not transition into the low state for trajectories of type I, II and VI. The colors correspond to those in Fig. 6.2.

the inclination of the system to be in either state. By increasing $\left|f_{i}\right|$ at constant $f_{o}$ (trajectory type $\mathrm{Vb}$ ) the system also undergoes transitions to the low state at the expected value of $a \approx 0.51$ (except for 1 out of 5 measurements). This transition however, is incidentally at a lower $\mathrm{Ta}=6.4 \cdot 10^{11}$ than the previously observed boundary of $\mathrm{Ta}=(7.7 \pm 0.2) \cdot 10^{11}$ for trajectories of constant Ta.

So far we have approached the right boundary in the phase space only from the high state. By now first traversing it from the right and then reversing the direction of $a$, we can investigate the system jumping back to the high state: does hysteresis of the transition position occur and can we quantify it? This was done by following trajectories of type VI at several different Taylor numbers. In the phase space we can see that for most of the measurements the systems undergoes transitions back to the high state slightly later (i.e. at higher $a$ ) when increasing $a$ again. In Fig. 6.5 an example of a measurement is shown where the direction of $a$ is changed three times. The difference in the value of $a$ between the jumps back and forth was less than 0.015 here, while in general it was smaller than 0.030 . We conclude that there is some hysteresis, which is only just revealed within the variation of the jump positions between measurements. 


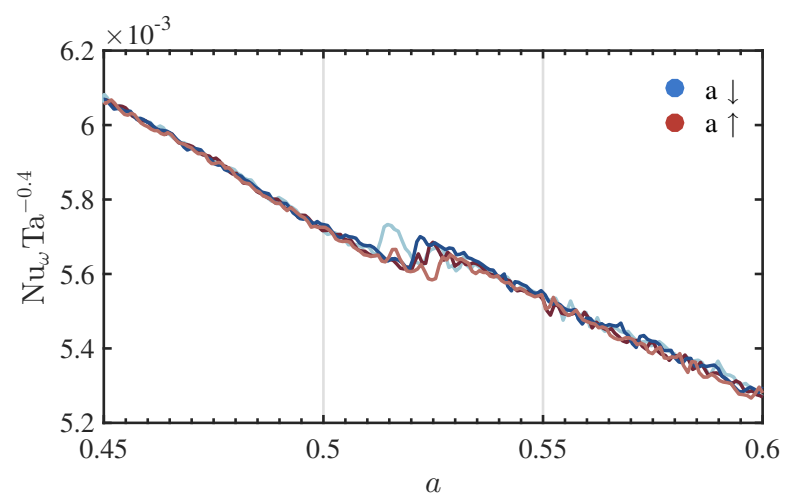

Figure 6.5: Global torque signal of one measurement following a type IV trajectory at $\Delta f=$ $15 \mathrm{~Hz}$, decreasing and increasing $a$ twice, showing hysteresis in the transitional $a$-value.

Having filled the phase space, we can now see how the boundaries of the multiple states region behave. The previously found range of $0.17<a<0.51$ holds for a large range of Ta, and only widens slightly with decreasing Ta below $\mathrm{Ta}=10^{12}$. When looking at the larger values of the Taylor number, there is no indication of the multiple states region closing up. From this, we expect the multiple states to exist (far) beyond $\mathrm{Ta}=10^{13}$.

\subsection{Flow structures in the BTTC setup with $\Gamma=18.3$}

Having shown the dependence of the existence of multiple states on the rotation ratio and driving strength, we will now turn our attention to another very important parameter: the aspect ratio $\Gamma$ of the system. It is straightforward to see that the aspect ratio will strongly influence the roll structures of the system. But what do the roll structures corresponding to the different states actually look like? That is what we aim to answer in this section. In chapter 5 we have mapped the roll structures for increasing and decreasing $a$ in the $\mathrm{T}^{3} \mathrm{C}$ setup, showing that 8 and 6 -roll states can exist for an aspect ratio of 11.7, with the 6-roll state corresponding to the non-default 'low' torque state. Here we performed similar experiments in the BTTC setup with an aspect ratio of 18.3 , with a finer axial resolution to individually resolve the expected larger number of rolls.

First, let us look at measurements of the azimuthal velocity at a single positions for both setups, see Fig. 6.6(a) and (b). In both cases the velocity bifurcates, showing very stable multiple states. For $a>0.51$ the velocity fluctuates strongly, which is not suggestive of a stable, well-defined flow state. However, the velocity was measured at the middle of the gap, and it could be possible that coherent structures still existed closer to the inner cylinder [123]. Now, as can be seen in 

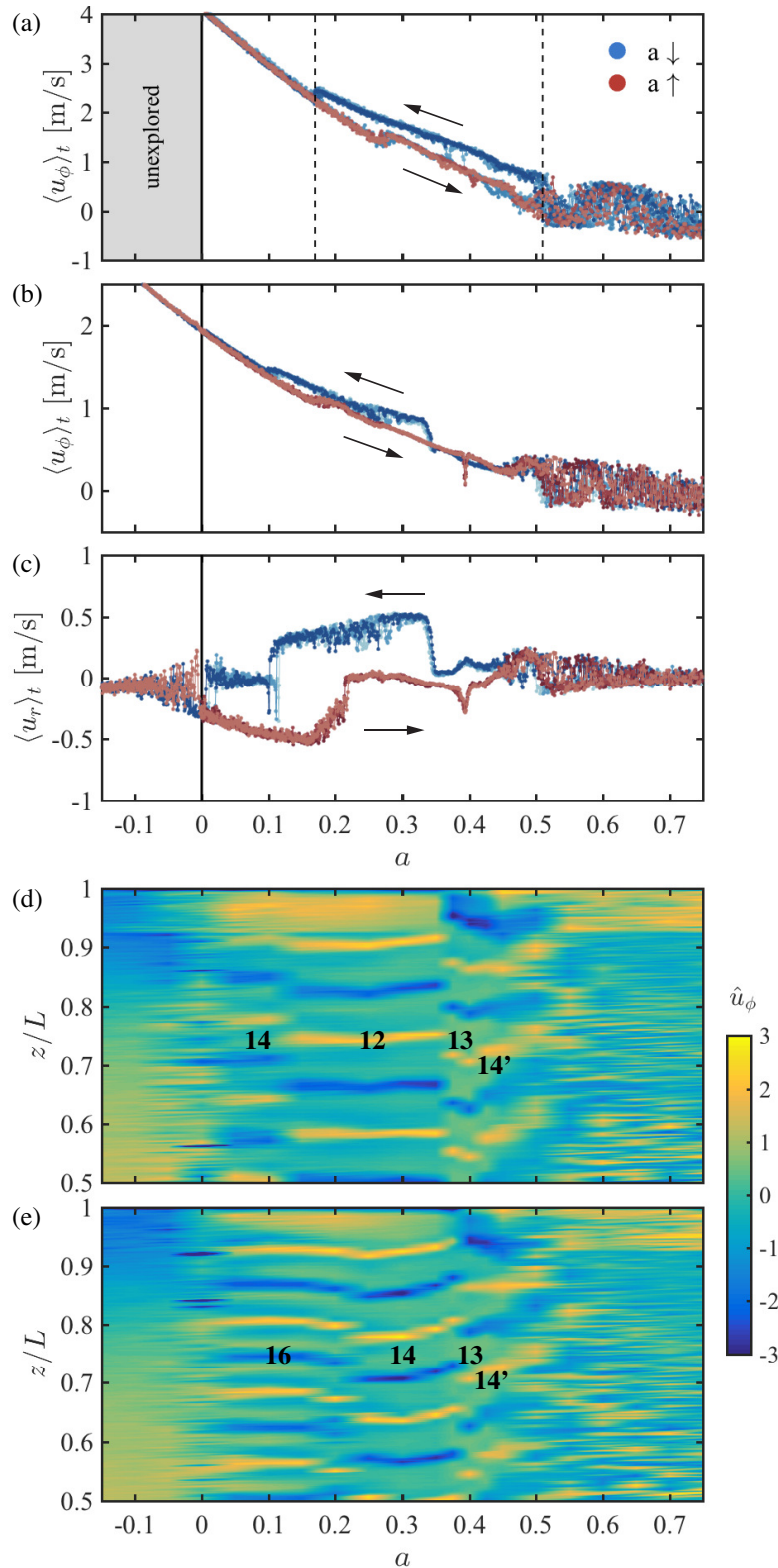

Figure 6.6: Local velocity and flow structures. (a) Azimuthal velocity measured in the $\mathrm{T}^{3} \mathrm{C}$ setup at $r=\left(r_{i}+r_{o}\right) / 2$ and $z / L=0.5$ as a function of $a$ for increasing and decreasing $a$ at $\mathrm{Ta} \approx 10^{12}$ (data from chapter 5). (b) and (c) Azimuthal and radial velocity, respectively, in the BTTC setup at $r=\left(r_{i}+r_{o}\right) / 2$ and $z / L=0.75$ at Ta $=3 \cdot 10^{10}$. (d) and (e) Axial scans of the standardized azimuthal velocity in the BTTC setup for decreasing (d) and increasing (e) $a$ at $\mathrm{Ta}=3 \cdot 10^{10}$. The standardized velocity is $\hat{u}_{\phi}=\left(\left\langle u_{\phi}\right\rangle_{t}-\left\langle u_{\phi}\right\rangle_{z}\right) / \sigma_{a}\left(u_{\phi}\right)$ where $\sigma_{a}\left(u_{\phi}\right)$ is the standard deviation of $u_{\phi}$ for each a, and \langle\rangle$_{x}$ stands for averaging over $x$. The bold numbers indicate the number of rolls the systems contains over the full height, with the accent denoting a reversed roll direction. 
Fig. 6.6(c), the radial velocity is a stronger indicator of the multiple states than the azimuthal velocity. While only two branches were observed, one for increasing $a$ and one for decreasing $a$, there are strong jumps of the velocity within these branches. This indicates that more than one state is possible in either branch.

By performing the aforementioned axial scans of the azimuthal velocity, we saw that this indeed is the case. Fig. 6.6(d) shows that for $a$ outside $[0,0.5]$ there are no clearly defined structures, but that within that range, a multitude of states is accessible. To count the number of rolls, we used the following concept: in between a pair of counter-rotating rolls, either high-velocity fluid from the inner cylinder or low-velocity fluid from the outer cylinder is advected, creating maxima and minima in the standardized velocity. From this we deduced that one roll sits between every minimum and maximum. With this knowledge, we counted 12, 13, and 14 rolls for different regions of $a$, for the measurement with decreasing $a$.

The case of increasing $a$, Fig. 6.6(e), also shows a myriad of accessible states, with 13, 14 and 16 rolls. Curiously, for $a$ within the range of $[0,0.4]$ the two branches of increasing and decreasing $a$ are never in the same roll state, again showing the importance of the history of the system. By following different trajectories in phase space, one can end up in different states, which are stable for at least multiple hours. Apparently, information about the history of the flow is not destroyed by the strong turbulence.

The average aspect ratio of the observed rolls were 1.53, 1.41, 1.31 and 1.14 for 12, 13, 14 and 16 rolls, respectively. The aspect ratios of the rolls observed in the $\mathrm{T}^{3} \mathrm{C}$ with smaller system aspect ratio of $\Gamma=11.7$ were 1.96 and 1.46 , measured however at a larger Taylor number of $\mathrm{Ta}=10^{12}$. The difference in aspect ratio can be explained by a trend of increasing aspect ratio with Reynolds number (and Taylor number) [109], although relatively stronger end effects in the $\mathrm{T}^{3} \mathrm{C}$ setup with smaller $\Gamma$ could also play a role. The fact that more roll states can be observed in the BTTC setup can be easily explained by the smaller energy barrier the system has to overcome to change the roll state. This energy barrier is smaller because the differences between the roll aspect ratios are smaller for the larger system aspect ratio $\Gamma$.

Signs of symmetry-breaking appear in Fig. 6.6(e) for $a$ in the range [0.2,0.4], where the top roll slowly changes aspect ratio, previously also observed in the $\mathrm{T}^{3} \mathrm{C}$ setup. More strikingly, at $a=0.4$ a roll state with an odd number of rolls seems to have appeared, which is very unexpected considering the symmetric boundary conditions of the system. We will now look into this phenomenom more closely using Fig. 6.7. By comparing the case of $a=0.375$ and $a=0.425$, we found that the system was in the same roll state of 14 vortices for these values of $a$, however with reversed flow direction of the rolls. We remind the reader that in this setup the end plates rotate with the outer cylinder, and it can be expected that the preferred roll direction at the boundaries depends on the rotation ratio $a$. In between 


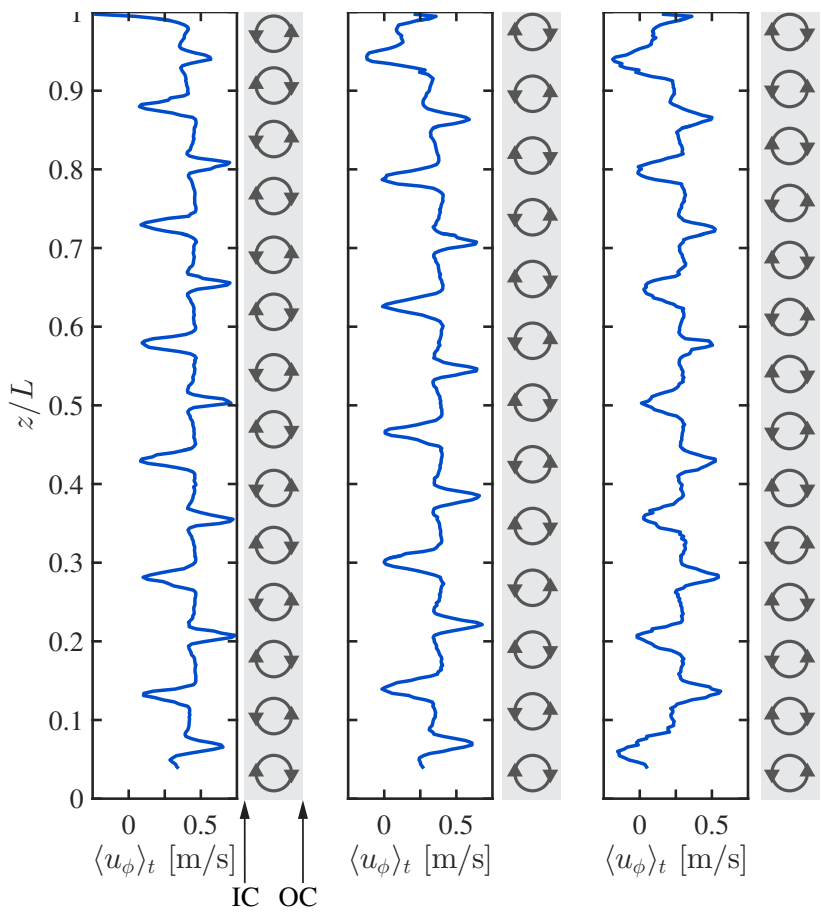

Figure 6.7: Azimuthal velocity profiles at Ta $=3 \cdot 10^{10}$ as a function of height $z / L$ for $a=$ $0.375, a=0.4$ and $a=0.425$, with increasing $a$. The data are from Fig. 6.6(e) but here shown for the full height of the system. The gray circles with arrows schematically show the rolls and their flow directions. Here the inner cylinder (IC) is on the left, the outer cylinder (OC) is on the right, and the convention of positive IC rotation is used.

these two cases, at $a=0.4$, both states are seemingly not favorable and the system reacts by first changing (in this particular case) the flow direction of the top roll of the system to the new boundary condition. Now that the top and bottom rolls rotate in the same direction, only an odd number of rolls can be supported in the system. This state has the center of the middle roll at $z / L=0.5$, making the term 'antisymmetrical' appropriate. Moving on to $a=0.425$, also the bottom roll in the system has adapted, arriving again at the expected symmetrical roll structure. In the case of decreasing $a$, the odd 13-roll state can also be observed, although the system does not revert to the 14-roll state until much later (smaller) $a$.

To our knowledge this is the first time a stable antisymmetrical strongly turbulent roll structure has been observed in a symmetrical Taylor-Couette setup. More generally, symmetry-breaking has been observed before in von Kármán flow with curved propellors [132]. 


\subsection{Conclusions}

To answer the question we ended the introduction with: multiple states in highly turbulent Taylor-Couette flow are very robust. In this chapter their existence was observed for a Taylor number range of almost two decades, and it can be expected that they persist for $\mathrm{Ta}=10^{13}$ and beyond. Although their existence is robust, to reliably reach the less-likely low torque state in our $\mathrm{T}^{3} \mathrm{C}$ setup, complex paths in phase space can be necessary. When a path of constant Ta (i.e. constant difference of rotation rates) is taken, a minimum Taylor number of $\mathrm{Ta}=(7.7 \pm 0.2) \cdot 10^{11}$ is needed to observe multiple states.

The rotation ratio $a$ is found to be the main control parameter for the transition; only a very weak dependence of the transitional $a$-values on the Taylor number was observed. Traversing the boundaries of the phase space with nonconstant $a$ and Ta also does not significantly influence the transitional $a$ value.

The robustness also reflects in the measurements we performed in a different setup with a larger aspect ratio. Here, the phenomena are even richer, although the same principles hold, namely that the system is hysteretic and can be in multiple stable turbulent states for the same driving parameters. For the larger aspect ratio, the system can be in a larger number of different roll states. Additionally, for the first time in turbulent TC flow, an antisymmetrical roll state with an odd number of rolls has been observed.

A theoretical understanding of the values of $a$ at which the systems transitions between states remains elusive, although we have shown here that the transitional $a$ values strongly depend on the aspect ratio of the system. Another interesting opportunity for further research is the probabilistic nature of the bifurcations.

With this work we have continued the investigation into the manifestation and selectability of coherent states in highly turbulent flows, which is important for understanding and predicting large-scale flows in nature and industry. 


\section{7}

\section{Taylor-Couette turbulence at radius ratio $\eta=0.5$ : scaling, flow structures and plumes*}

Using high-resolution particle image velocimetry we measure velocity profiles, the wind Reynolds number and characteristics of turbulent plumes in Taylor-Couette flow for a radius ratio of 0.5 and Taylor number of up to $6.2 \cdot 10^{9}$. The extracted angular velocity profiles follow a log-law more closely than the azimuthal velocity profiles due to the strong curvature of this $\eta=0.5$ setup. The scaling of the wind Reynolds number with the Taylor number agrees with the theoretically predicted 3/7-scaling for the classical turbulent regime, which is much more pronounced than for the well-explored $\eta=0.71$ case, for which the ultimate regime sets in at much lower Ta. By measuring at varying axial positions, roll structures are found for counter-rotation while no clear coherent structures are seen for pure inner cylinder rotation. In addition, turbulent plumes coming from the inner and outer cylinder are investigated. For pure inner cylinder rotation, the plumes in the radial velocity move away from the inner cylinder, while the plumes in the azimuthal velocity mainly move away from the outer cylinder. For counter-rotation, the mean radial flow in the roll structures strongly affects the direction and intensity of the turbulent plumes. Furthermore, it is experimentally confirmed that in regions where plumes are emitted, boundary layer profiles with a logarithmic signature are created.

${ }^{*}$ Submitted as: Roeland C. A. van der Veen, Sander G. Huisman, Sebastian Merbold, Uwe Harlander, Christoph Egbers, Detlef Lohse and Chao Sun, Taylor-Couette turbulence at radius ratio $\eta=0.5$ : scaling, flow structures and plumes. Measurements by RVDV, SH and SM, analysis and writing by $\mathrm{RVDV}$, discussion of the results and proofreading of the manuscript by everyone. 


\subsection{Introduction}

The paradigmatic Taylor-Couette (TC) flow has long been a flow configuration of great interest to fluid dynamicists. It consists of flow between two coaxial cylinders that can independently rotate, see Fig. 7.1. This system has been used extensively as a model in fluid physics because it is a closed system, has a relatively simple geometry and therefore has multiple symmetries. After early investigations $[7,8,150]$, Wendt [134] started studying turbulence in this system. For a further historical overview, the reader is referred to Donnelly [133]. For a review on TC flow at the onset of instabilities and slightly above, see Fardin et al. [104]. The state-of-the-art of high Reynolds number Taylor-Couette turbulence is treated by Grossmann, Lohse, and Sun [11].

The two geometrical control parameters of TC flow are the ratio $\eta$ of the inner and outer cylinder radius and the aspect ratio $\Gamma=L / d$, where $d$ is the gap width and $L$ the height of the cylinders. In this chapter we use a small radius ratio of $\eta=0.5$, corresponding to a relatively wide gap. The vast majority of existing work (see overview by Dubrulle et al. [74] and review by Grossmann et al. [11]) focuses on a radius ratio of 0.71 or higher. Lower $\eta$ experiments concern the Rayleighstable regime [136], mean flow and turbulence characteristics [138] and global torque measurements [107]. In addition, numerical work has been done for $\eta=0.5$ $[88,109,141]$. The radius ratio is a key control parameter [12] in TC flow and it has been found that a low radius ratio Taylor-Couette system behaves differently as compared to higher $\eta$ setups in several aspects, which will be outlined below. The underlying reason for the different behavior is the strong boundary layer asymmetry. Because the ratio of inner and outer boundary layer thicknesses scales as $\eta^{3}$ [12], a strong asymmetry between the inner and outer boundary layer exists for small radius ratio.

As dimensionless control parameters of the system we use the (negative) rotation ratio $a=-\omega_{o} / \omega_{i}$ and the Taylor number [12]:

$$
\mathrm{Ta}=\frac{(1+\eta)^{4}}{64 \eta^{2}} \frac{\left(r_{o}-r_{i}\right)^{2}\left(r_{i}+r_{o}\right)^{2}\left(\omega_{i}-\omega_{o}\right)^{2}}{v^{2}}
$$

where $\omega_{i, o}$ are the angular velocities of the inner and outer cylinder, $r_{i, o}$ are the inner and outer cylinder radii, $\eta=r_{i} / r_{o}$ is the radius ratio, and $v$ is the kinematic viscosity. The driving of the system can also be described by the shear Reynolds number [74]:

$$
\operatorname{Re}_{\mathrm{S}}=\frac{2 r_{i} r_{o}\left(r_{o}-r_{i}\right)}{v\left(r_{o}+r_{i}\right)}\left|\omega_{o}-\omega_{i}\right|
$$

for which $\operatorname{Re}_{\mathrm{S}} \propto \mathrm{Ta}^{1 / 2}$.

When increasing the driving strength (i.e. the Taylor number), TC flow first gradually undergoes a transition from a purely azimuthal, laminar state to a state 


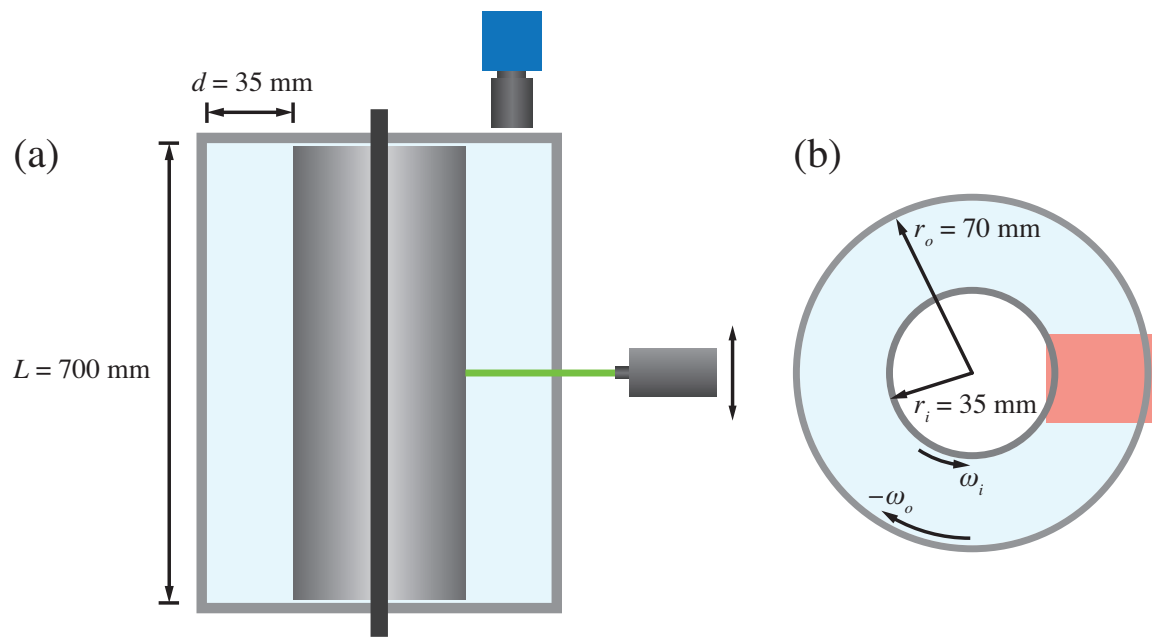

Figure 7.1: (a) Schematic of the vertical cross-section of the Taylor-Couette setup, showing the horizontal laser sheet which is imaged from above trough the transparent top plate. The laser can be traversed vertically. (b) Horizontal cross-section of the setup. The red rectangle represents the typical field of view.

where the bulk becomes turbulent while the boundary layers still remain laminar. The latter state is called the classical regime [14]. By further increasing Ta, the ultimate regime $[118,125,146]$ is reached, in which also the boundary layers are turbulent. The signature of these turbulent boundary layers are logarithmic velocity profiles which have recently been found for a radius ratio of $\eta=0.716$ [87] and for $\eta=0.909$ [151]. Grossmann et al. [152] have derived theoretical velocity profiles, and found that the angular velocity profile follows a log-law more closely than the azimuthal velocity profile, an effect that is more pronounced with the stronger asymmetry for smaller $\eta$. In section 7.3.1 we set out to investigate the correspondence of experimentally measured angular and azimuthal velocity profiles to a log-law for $\eta=0.5$ and Taylor numbers up to $6.2 \cdot 10^{9}$ at the onset of the ultimate turbulent regime. Furthermore, the velocity profiles are compared to existing experimental and numerical work.

In addition to affecting the velocity profiles, the radius ratio strongly influences the transitional Taylor number for the ultimate regime $[88,90,107,126]$. For $\eta=0.5$ the ultimate regime does not start before $\mathrm{Ta}=10^{10}[88,107]$, whereas the transition for a higher radius ratio of $\eta=0.71$ already occurs at $\mathrm{Ta}=5 \cdot 10^{8}[88,127]$. Very different scaling of the angular momentum transfer and the "wind" in the gap with the driving paramater Ta exist for the classical and ultimate regime [14, 118]. The late onset of the ultimate regime for $\eta=0.5$ gives us the opportunity to analyze the scaling of the wind Reynolds number with Taylor numbers in the classical TC regime, up to the onset of the ultimate regime and compare it with theoretical 
predictions (section 7.3.2).

Another area where the influence of the radius ratio is apparent, is the angular momentum transport trough the gap. This transport is a key response parameter of the system and is directly connected to the torque required to maintain constant cylinder velocities [12]. The radius ratio strongly influences the rotation ratio for which optimal momentum transport occurs [90, 107, 127]. For example, the optimal momentum transport occurs at $a_{\text {opt }} \approx 0.33$ for $\eta=0.714$ [127] whereas it is $a_{\mathrm{opt}} \approx 0.20$ for $\eta=0.5$ [107].

Roll structures play an important role in the momentum transport in the Taylor-Couette system, as one can see in chapter 5 and Refs. [10, 83, 124, 143]. With increasing Taylor number, the system undergoes transitions from a purely laminar state to having steady, coherent Taylor vortices, to having modulated, wavy Taylor vortices which eventually evolve into chaotic turbulent Taylor vortices [83]. But even in the ultimate regime, for some $a>0$, it was found that remnants of these rolls are present in time-averaged quantities (chapter 5 and Refs. [124, 144]), whereas for $a=0$ (pure inner cylinder rotation) these rolls vanish for large Taylor number [135]. In section 7.3.3 we will characterize the roll structures for $\eta=0.5$ at both pure inner cylinder rotation $a=0$ and slight counter-rotation $a=0.2$, at which optimal transport occurs.

It was recently proposed that the logarithmic velocity profiles in ultimate TC flow are triggered by turbulent plume ejection [124, 153, 154], which in turn is regulated by the relative strength of the axial and radial mean flow. Using timeresolved azimuthal and radial velocity field measurements at several heights in the setup we will investigate the connection between the roll structures that generate strong radial and axial flow, and the turbulent plumes that emanate from either the inner or outer cylinder (section 7.3.4). Futhermore, in section 7.3.5 we will quantify how these plumes affect the logarithmic nature of the velocity profiles.

\subsection{Setup \& explored parameter space}

\subsubsection{Setup}

The experiments were carried out in the Cottbus Taylor-Couette facility [107, 155]. The inner and outer cylinder radii are $r_{i}=35.0 \pm 0.2 \mathrm{~mm}$ and $r_{o}=70.0 \pm 0.2 \mathrm{~mm}$, respectively, the height of the setup is $L=700 \mathrm{~mm}$. This gives a radius ratio of $\eta=0.5$ and an aspect ratio of $\Gamma=20$. The cylindricities of the cylinders that were used are $0.4 \mathrm{~mm}$ and $0.3 \mathrm{~mm}$ for the inner and outer cylinder, respectively. The maximum rotation rates are $5 \mathrm{~Hz}$ for both the inner and outer cylinder.

The end plates rotate with the outer cylinder. The outer cylinder and the top plate are transparent, making the setup ideally suited to be used in combination 

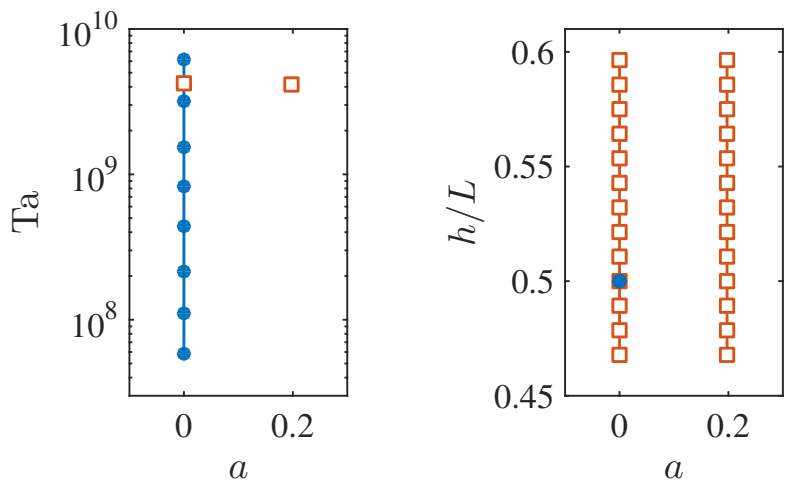

Figure 7.2: The explored parameter space. The Taylor number (Ta) is varied for inner cylinder rotation $(a=0)$, and axial scans have been performed for (negative) rotation ratios $a=0$ and $a=0.2$.

with particle image velocimetry (PIV). A high-resolution PIV camera (LaVision Imager sCMOS) with a resolution of $2560 \times 2160$ pixels and a frame rate of $50 \mathrm{~Hz}$ is installed above the top end plate, pointing downwards. Water is used at the working fluid $\left(20^{\circ} \mathrm{C}, v=1.0 \cdot 10^{-6} \mathrm{~m}^{2} / \mathrm{s}\right)$. The water contains fluorescent particles (Dantec Dynamics, PMMA-RhB, 1-20 $\mu \mathrm{m}$ ) with a maximum Stokes number of $\mathrm{St}=\tau_{p} / \tau_{\eta} \approx 10^{-4} \ll 1$, which means that they faithfully follow the flow and can be considered as tracer particles. The flow is illuminated by a horizontal light sheet from a high-powered pulsed Nd:YLF dual cavity laser (Litron LDY303HE). Because of the high-resolution PIV camera, very high resolution measurements of the flow fields can be achieved. The imaging of the full width of the gap combined with a vector grid of $16 \times 16$ pixels with $50 \%$ overlap results in a velocity vector spacing of $0.13 \mathrm{~mm}$. The PIV system is operated in dual frame mode, allowing for an interframe time $\Delta t$ smaller than the inverse frame rate. The PIV image pairs are processed using LaVision DaVis software, after which the flow fields are transformed to the radial velocity $u_{r}(\theta, r, z, t)$ and the azimuthal velocity $u_{\theta}(\theta, r, z, t)$.

\subsubsection{Explored parameter space}

The parameter space that was explored in this chapter is shown in Fig. 7.2. The first set of measurements that is treated here consists of varying the Taylor number between $\mathrm{Ta}=5.8 \cdot 10^{7}$ and $\mathrm{Ta}=6.2 \cdot 10^{9}$ (corresponding to a shear Reynolds number of $\operatorname{Re}_{\mathrm{S}}=6.0 \cdot 10^{3}$ to $\left.6.2 \cdot 10^{4}\right)$ for inner cylinder rotation only $(a=0)$ at mid-height $(h=0.5 L)$. These measurements were performed as a single continuous experiment; the cylinder velocity was increased slowly between experiments and before each measurement approximately 10 minutes were taken to give the flow ample time to stabilize. Each measurement in this set consists of 10000 PIV image pairs, 

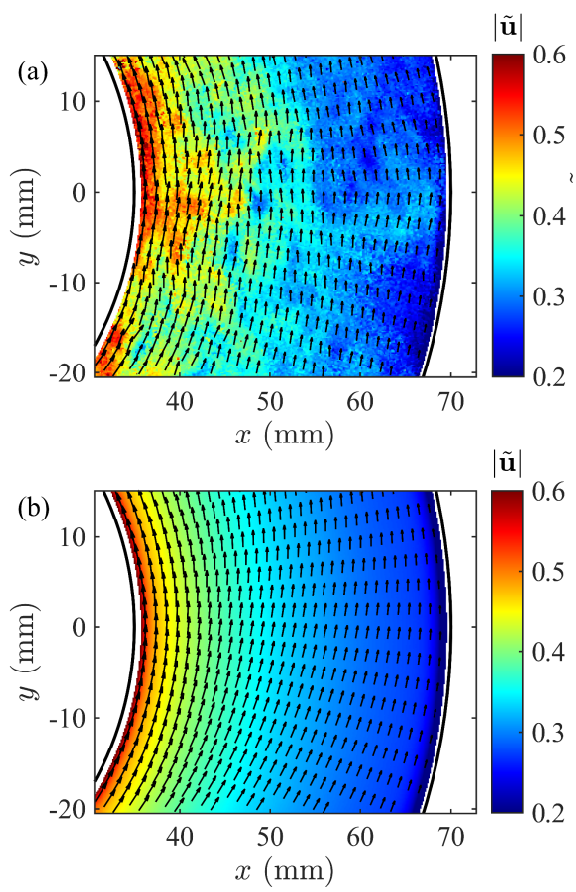
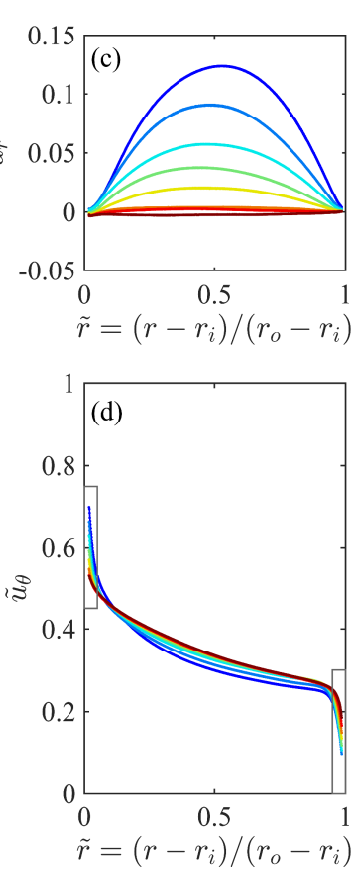
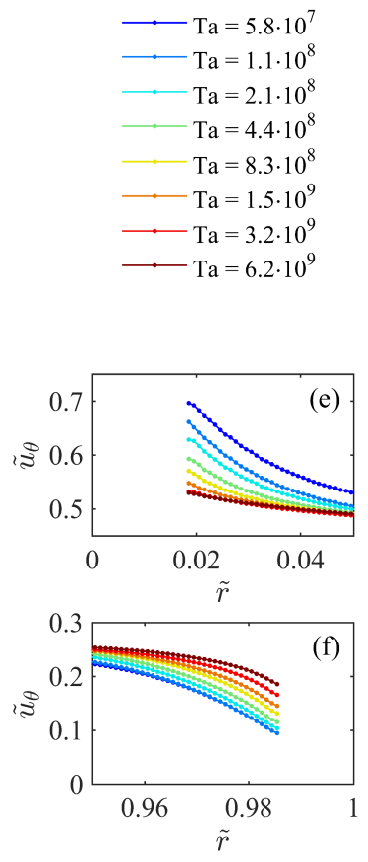

Figure 7.3: Overview of the flow profiles for varying Taylor number with pure inner cylinder rotation $a=0$ at mid-height $h / L=0.5$. (a) Snapshot of the flow field for Ta $=6.2 \cdot 10^{9}$. The colors and lengths of the arrows indicate the norm of the velocity $|\tilde{\mathbf{u}}|$. (b) Averaged flow field over 10000 PIV image pairs at Ta $=6.2 \cdot 10^{9}$. (c) Radial velocity profiles across the gap of the TC apparatus, normalized by the inner cylinder velocity. For lower Taylor numbers there is still a strong radial flow, which can be attributed to the presence of Taylor vortices. (d) Azimuthal velocity profiles normalized by the inner cylinder velocity. For increasing Taylor number, the profiles become flatter and the boundary layers steeper. (e) and (f) Magnification of the azimuthal velocity profiles in (d) close to the cylinders.

which were recorded at 25 frames per second. This corresponds to 235 and 2418 cylinder revolutions for $\mathrm{Ta}=5.8 \cdot 10^{7}$ and $\mathrm{Ta}=6.2 \cdot 10^{9}$, respectively.

In addition to investigating the Taylor number dependence, the height of the laser sheet was varied to characterize the axial dependence of the flow field. At 13 different heights with $7.5 \mathrm{~mm}$ spacing, 5000 image pairs of the velocity field were recorded. This was done at $\mathrm{Ta}=4.2 \cdot 10^{9}$ for both pure inner cylinder rotation and optimal [107] counter-rotation $a=0.2$. 


\subsection{Results}

\subsubsection{Azimuthal and angular velocity profiles}

In this part the properties of flow profiles for varying Taylor number will be investigated. An example of an instantaneous flow field is shown in Fig. 7.3(a), while the averaged field is shown in (b). From (c) it is apparent that at this specific axial position (mid-height) a significant mean radial flow still exists for the lower Taylor numbers up to approximately $10^{9}$. These are Taylor vortices, which at $a=0$ disappear for higher Taylor numbers [135]. With increasing Taylor number the azimuthal velocity profile in the bulk becomes flatter (Fig. 7.3(d-f)), but it is still apparent that the strong curvature of a radius ratio of $\eta=0.5$ creates a significant asymmetry between the inner and outer boundary layer.

In Fig. 7.4 profiles up to the middle of the gap are shown, normalized to the wall normal distance $y^{+}$from both the inner and outer cylinder, and to the azimuthal velocity $u^{+}\left((\mathrm{a})\right.$ and (b)) and angular velocity $\omega^{+}((\mathrm{c})$ and (d)). The wall normal distance, azimuthal velocity and angular velocity for the inner cylinder are defined as:

$$
\begin{aligned}
y^{+} & =\frac{r-r_{i}}{\delta_{v, i}} \\
u^{+} & =\frac{u_{\theta}\left(r_{i}\right)-u_{\theta}(r)}{u_{\tau, i}} \\
\omega^{+} & =\frac{\omega\left(r_{i}\right)-\omega(r)}{u_{\tau, i} / r_{i}} ;
\end{aligned}
$$

The same parameters for the outer cylinder are defined as:

$$
\begin{aligned}
y^{+} & =\frac{r_{o}-r}{\delta_{v, o}} \\
u^{+} & =\frac{u_{\theta}(r)-u_{\theta}\left(r_{o}\right)}{u_{\tau, o}} \\
\omega^{+} & =\frac{\omega(r)-\omega\left(r_{o}\right)}{u_{\tau, o} / r_{o}}
\end{aligned}
$$

with the viscous length scale $\delta_{v, i}=v / u_{\tau, i}$, the friction velocity $u_{\tau, i}=\sqrt{\tau_{w, i} / \rho}$ containing the wall shear stress $\tau_{w, i}=T / 2 \pi r_{i}^{2} L$ with the torque $T$, where the subscript $i$ may be replaced by $o$. The torque values are from Ref. [107], where a (non-transparent) Taylor-Couette setup with identical dimensions is used. The local torque can be different from the average torque, following the large-scale roll structures. This causes the imperfect matching with the viscous sublayer $u^{+}=y^{+}$ and $\omega^{+}=y^{+}$, especially visible in Fig. 7.4(b) and (d). 

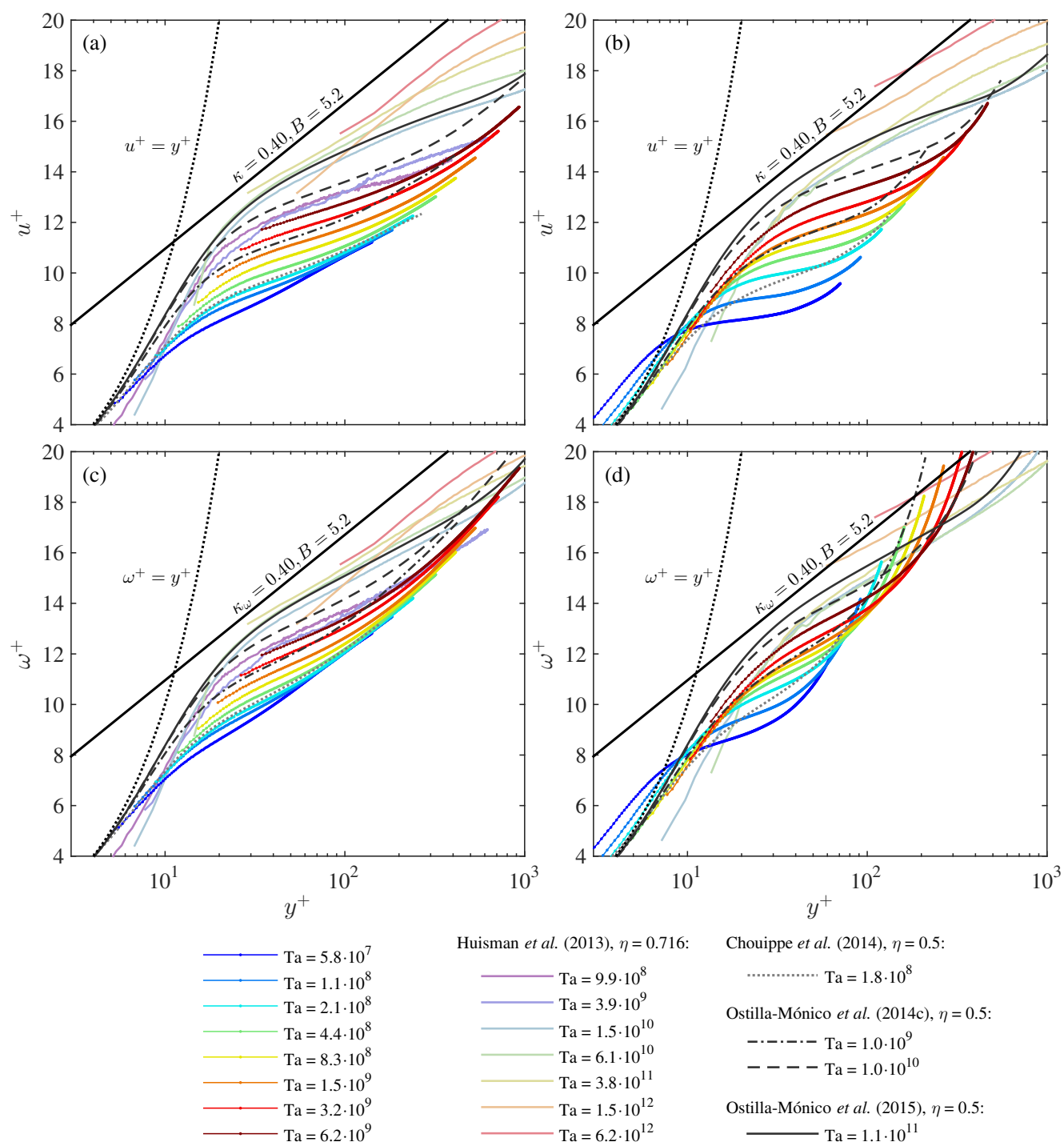

Huisman et al. (2013), $\eta=0.716$ :

Chouippe et al. (2014), $\eta=0.5$ :

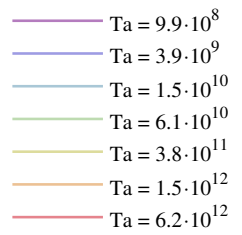

.............. Ta $=1.8 \cdot 10^{8}$

Ostilla-Mónico et al. (2014c), $\eta=0.5$ :

-.-.-.- $\mathrm{Ta}=1.0 \cdot 10^{9}$

$----\mathrm{Ta}=1.0 \cdot 10^{10}$

Ostilla-Mónico et al. (2015), $\eta=0.5$ :

$-\mathrm{Ta}=1.1 \cdot 10^{11}$

Figure 7.4: Velocity profiles for inner cylinder rotation $a=0$ and varying Taylor number. See main text for the definitions of $u^{+}, \omega^{+}$and $y^{+}$. (a) Azimuthal velocity profiles near the inner cylinder $(\tilde{r} \in[0,1 / 2])$.(b) Azimuthal velocity profiles near the outer cylinder $(\tilde{r} \in$ $[1 / 2,1])$. (c) Angular velocity profiles near the inner cylinder $(\tilde{r} \in[0,1 / 2])$. (d) Angular velocity profiles near the outer cylinder $(\tilde{r} \in[1 / 2,1])$. All figures include the logarithmic law of the wall from Prandtl and von Kármán $u^{+}=1 / \kappa \ln y^{+}+B$ with the typical values of $\kappa=0.40$ and $B=5.2$ (see Ref. [156] and references therein), the viscous sublayer $u^{+}=y^{+}$, DNS data from Refs. [109], [88] and [151] at $\eta=0.5$ and measurement data from Ref. [87] at $\eta=0.716$. 
As the driving is increased, the profiles slowly approach the Prandtl-von Kármán log-law, although at these Taylor numbers the log layer is not yet fully developed. For comparison, profiles at higher Taylor numbers and aspect ratio of $\eta=0.716$ from Ref. [87] are also plotted. The data show good agreement with direct numerical simulations (DNS) at the same radius ratio $\eta=0.5$ from Chouippe et al. [109] at Ta $=1.8 \cdot 10^{8}$ and Ostilla-Mónico et al. at $\mathrm{Ta}=1.0 \cdot 10^{9}$ and $\mathrm{Ta}=1.0 \cdot 10^{10}[88]$ and at $\mathrm{Ta}=1.1 \cdot 10^{11}[151]$.

When comparing the azimuthal velocity $u^{+}$to the angular velocity $\omega^{+}$(i.e. (a) to (c) and (b) to (d)) it can be seen that the angular velocity profiles curve upwards more compared to the azimuthal ones. This is consistent with the theoretical argument of Grossmann et al. [152] that the angular velocity profile $\omega^{+}$is closer to a log-law than the azimuthal velocity profile $u^{+}$. The effect is much more pronounced for the smaller $\eta=0.5$ here as compared to the $\eta=0.716$ from Huisman et al. [87]. Note that, for example, the lower Taylor number $u^{+}$profiles at $\eta=0.716$ are above the profiles measured here, but that they cross when represented as $\omega^{+}$. This clearly shows the influence of the large curvature for small radius ratio TC setups.

\subsubsection{Wind Reynolds number}

The degree of turbulence of the wind in the gap of the cylinders, which measures the strength of the secondary flows $u_{r}$ and $u_{z}$, can be characterized by the wind Reynolds number. We use the standard deviation of the radial velocity to quantify the wind Reynolds number:

$$
\mathrm{Re}_{\mathrm{w}}=\sigma\left(u_{r}\right) d / v
$$

with the gap width $d=r_{o}-r_{i}$. In the analogy between TC and Rayleigh-Bénard flow, the unifying theory of Grossmann et al. [14] predicts that the wind Reynolds number in the classical turbulent regime scales as:

$$
\operatorname{Re}_{\mathrm{w}} \propto \mathrm{Ta}^{3 / 7}
$$

In contrast, in the ultimate turbulent regime the scaling was found to be $\mathrm{Re}_{\mathrm{w}} \propto$ $\mathrm{Ta}^{1 / 2}$ [118], which was confirmed experimentally by Huisman et al. [128] for TC flow with $\eta=0.716$ for Ta up to $6.2 \cdot 10^{12}$.

The highest Taylor number we achieve in the present measurements is $\mathrm{Ta}=$ $6.2 \cdot 10^{9}$, which for $\eta=0.5$ with the higher transitional Taylor number of $\mathrm{Ta}=10^{10}$ $[88,107]$ implies that we are in the classical turbulent regime. Therefore we expect the theoretically predicted 3/7-scaling to hold. This classical scaling was measured in Rayleigh-Bénard convection [146], but to our knowledge has not yet been confirmed for TC flow. 

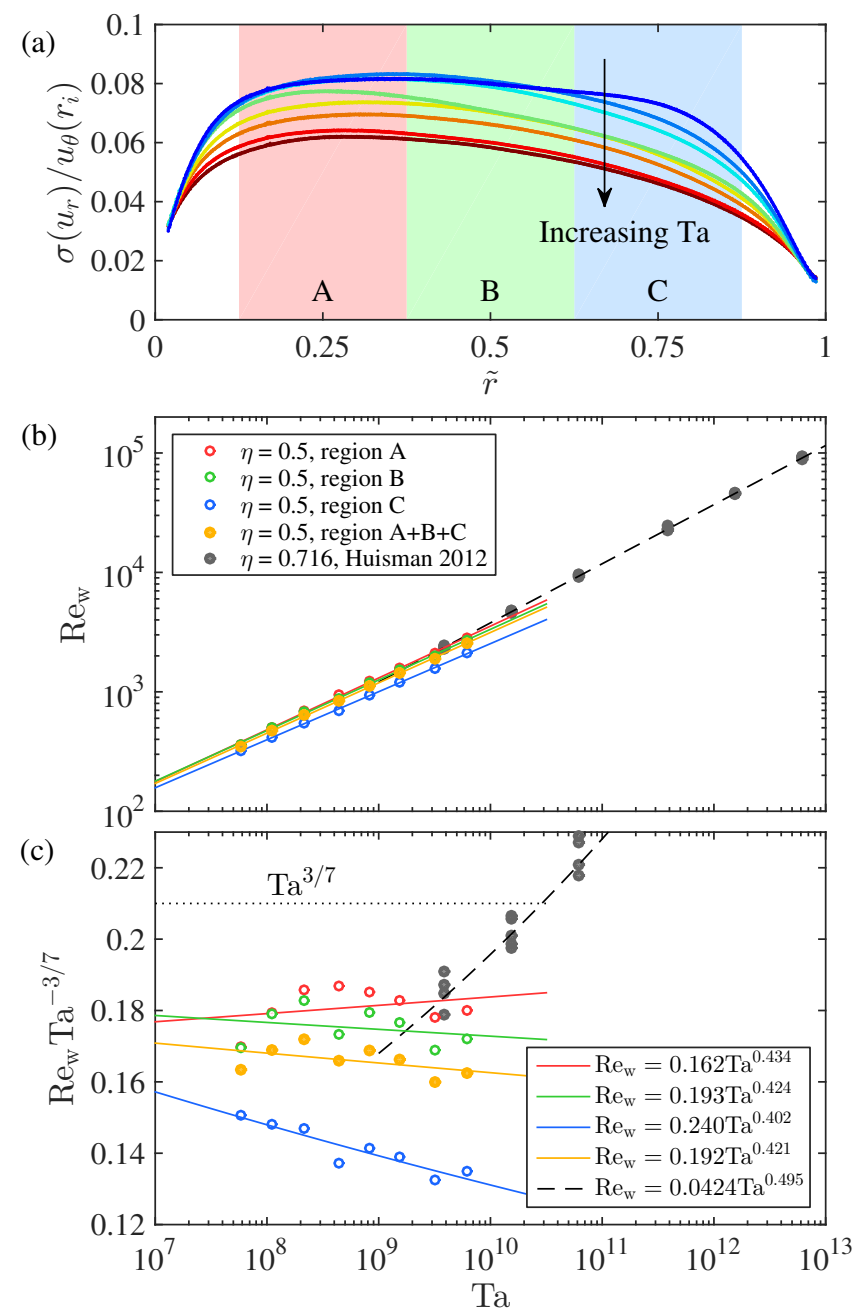

Figure 7.5: The scaling of the wind Reynolds number with Taylor number for inner cylinder rotation $a=0$. (a) The standard deviation of the radial velocity over 10000 frames (400 s) and the azimuthal direction, normalized by the inner cylinder velocity, as a function of the normalized radial position. See the legend in Fig. 7.3 or 7.4 for the values of the Taylor numbers. The three areas A $(\tilde{r} \in[1 / 8,3 / 8]), \mathrm{B}(\tilde{r} \in[3 / 8,5 / 8])$ and $\mathrm{C}(\tilde{r} \in[5 / 8,7 / 8])$ indicate regions over which $\sigma$ is averaged. (b) $\operatorname{Re}_{\mathrm{W}}$ versus Ta averaged over $\tilde{r}$-values corresponding to regions $\mathrm{A}, \mathrm{B}, \mathrm{C}$ and all three combined $(\tilde{r} \in[1 / 8,7 / 8])$. In addition, data from Huisman et al. [128] with their fit are shown. This data uses the equivalent to area $\mathrm{B}$ for averaging. (c) Data in (b) compensated by $\mathrm{Ta}^{3 / 7}$. The fits are $\mathrm{Re}_{\mathrm{W}}=0.162 \mathrm{Ta}^{0.434}$ for area A, $\mathrm{Re}_{\mathrm{W}}=0.193 \mathrm{Ta}^{0.424}$ for area $\mathrm{B}, \mathrm{Re}_{\mathrm{W}}=0.240 \mathrm{Ta}^{0.402}$ for area C and $\mathrm{Re}_{\mathrm{W}}=0.192 \mathrm{Ta}^{0.421}$ for the three areas combined. 
The wind Reynolds number $\mathrm{Re}_{\mathrm{w}}$ is extracted from the velocity field $u_{r}(\theta, r, z, t)$ by calculating the standard deviation $\sigma\left(u_{r}(r, \theta, t)\right)$ over time and the azimuthal direction, which is then averaged over a certain range in the gap, see Fig. 7.5. When looking at the shape of the $\tilde{r}$-dependence of $\sigma$, it is apparent that an asymmetry is present. The fluctuations exhibit a maximum around a quarter of the gap width away from the inner cylinder. It is likely that the strong curvature of the $\eta=0.5$ setup causes this assymmetry. Calculating the wind Reynolds number thus comes with an arbitrariness in the area to choose for averaging the profile of $\sigma(r)$, although excluding the boundary layers is reasonable because the interest lies in the convective bulk transport, as opposed to the diffusive transport in the boundary layers. The dependence of $\operatorname{Re}_{\mathrm{w}}(\mathrm{Ta})$ is shown in Fig. 7.5(b) for different regions of averaging. From Fig. 7.5(c) it can be seen that a clear power law scaling is present with an exponent that ranges from 0.402 to 0.434 , depending on the region of averaging. This is consistent with the theoretically predicted classical regime scaling of $3 / 7 \approx 0.429$ [14].

\subsubsection{Roll structures}

The phenomena of roll structures, turbulent plumes and logarithmic velocity profiles in Taylor-Couette flow are intimately connected [88, 124]. We will first show an example of roll structures that are observed in TC flow. Flow profiles at several heights for both $a=0$ and $a=0.2$ are measured, allowing us to visualize roll structures as shown in Fig. 7.6. At this reasonably high Taylor number of $\mathrm{Ta}=4.2 \cdot 10^{9}$, no clear roll structures exist for inner cylinder rotation only. The radial velocity shows some very faint indications of rolls, with a maximum value of $\tilde{u}_{r}=0.01$ in the center of the gap. In contrast, in the case of counter-rotation $(a=0.2)$, very strong roll structures can be seen. The fact that there are strong rolls at optimal counter-rotation and no clear structures at inner cylinder rotation only, is corroborated by recent work for higher radius ratio [124, 144], see also chapter 5 .

In the radial flow, between two rolls at approximately $h / L=0.51$, there is very strong outwards radial flow (see Fig. 7.6(e)). Specifically, the height $0.52 L$ corresponds to the bottom of a roll, while $0.50 \mathrm{~L}$ corresponds to the top of the roll below. Exactly in between the rolls at $0.51 \mathrm{~L}$, the radial flow surprisingly exhibits a local minimum. In Fig. 7.6(g,h) it can be seen that in the case of positive (outward) radial flow, inner cylinder velocity is advected outwards, shifting the profiles upwards at the inner half of the gap. For negative (inward) radial flow, the profiles shift downwards in the outer half of the gap. In summary, the roll structures are represented by a large secondary flow of $u_{r}$, which advects velocity from the cylinders and changes the azimuthal velocity profiles. In the following two subsections we will elaborate on the characteristics of turbulent plumes and their effect on the velocity profiles. 

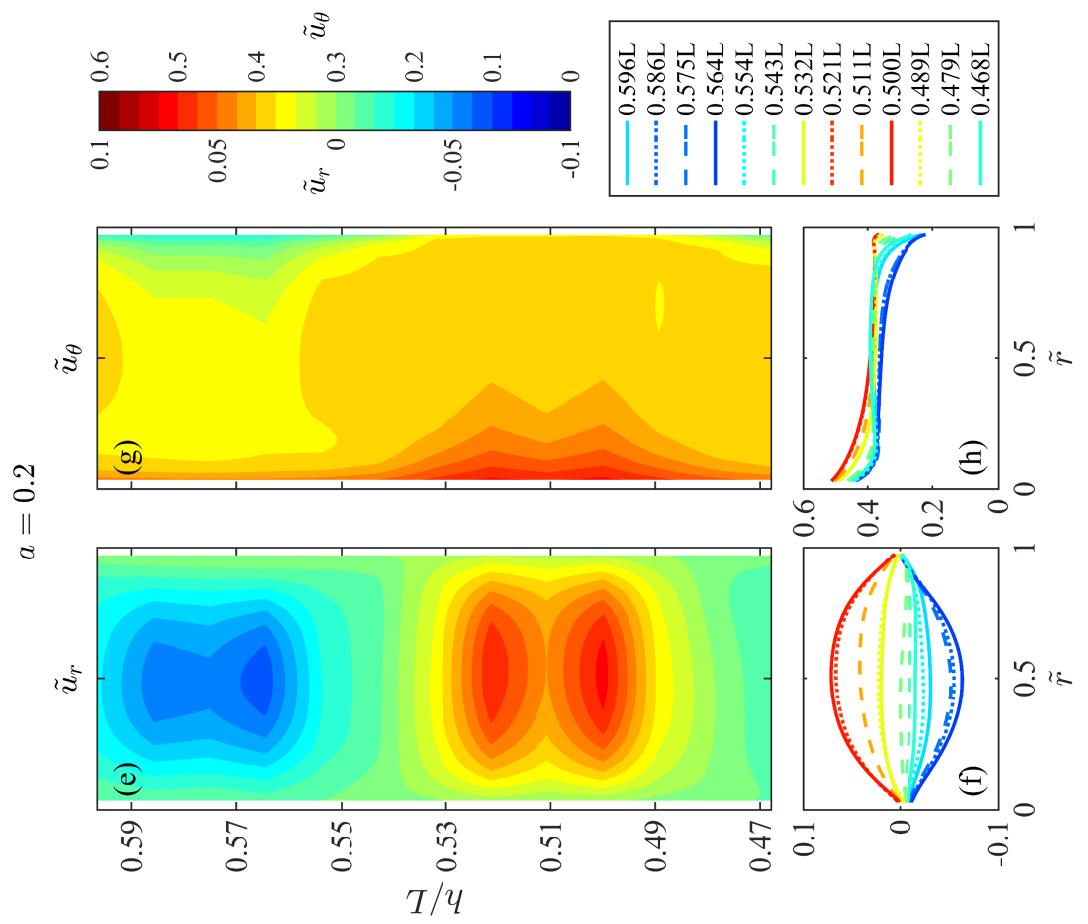

몰

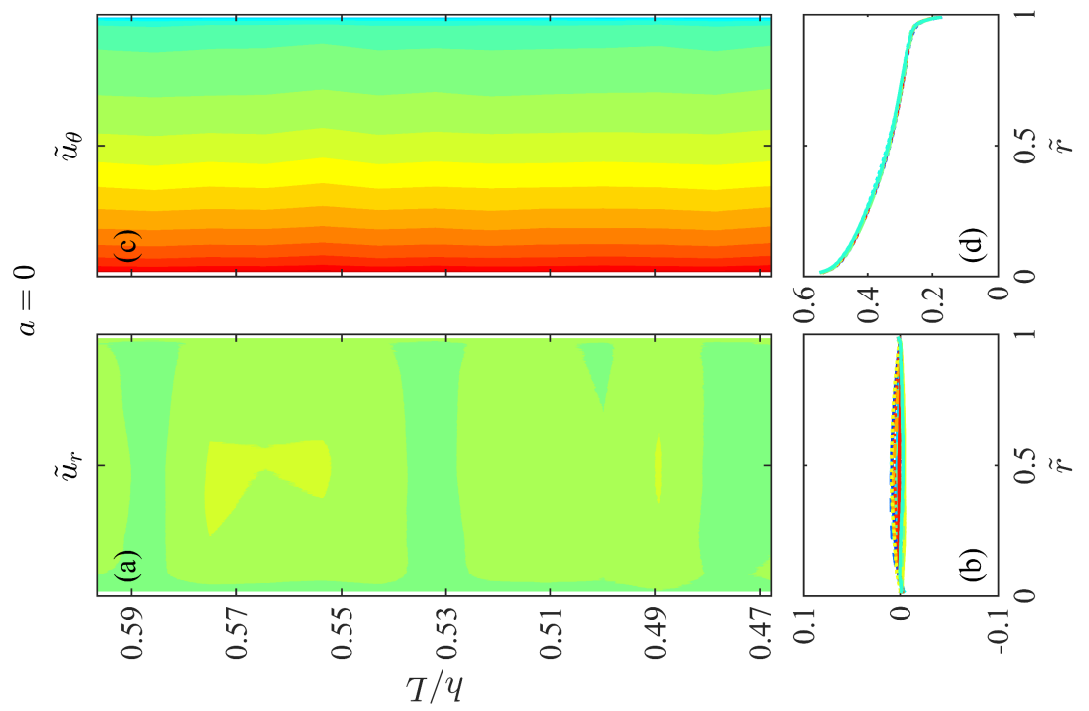

ర్త

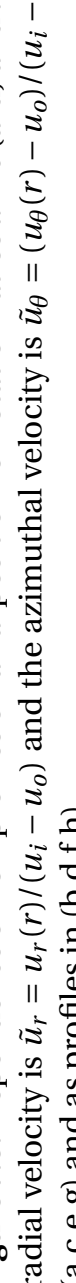




\subsubsection{Turbulent plumes}

In turbulent Taylor-Couette flow, structures detach from the boundary layers, called herring-bone streaks [141] or velocity plumes [124], in correspondence to the thermal plumes in the analogous turbulent Rayleigh-Bénard convection. These plumes in TC flow are large-scale spatial and temporal fluctuations in the velocity fields that detach from either the inner or outer cylinder and advect velocity from the respective cylinder. Because of the sufficiently high frame rate of 25 fps and large amount of data recorded in our experiments, these plumes can be resolved in time, and the typical velocity can be extracted. We are interested in understanding how these plumes are affected by roll structures and whether some scaling relation exists for the typical plume velocity as a function of the driving parameter Ta.

The time dependence of the profiles of the azimuthal and radial velocity can be represented by the spatio-temporal fields $u_{r}(r, t)$ and $u_{\theta}(r, t)$, with the value of the velocities represented by a color map, as shown in e.g. Fig. 7.7(a). In this way, structures or fluctuations of the profiles that propagate inwards or outwards become visible as diagonal lines. It has to be stressed that these diagonals do not represent a nonzero mean radial flow, but only the fluctuations on these profiles that travel outward or inward. In order to assign a velocity to these plumes we employ an analysis which is based on applying the following affine shearing transformation to the functions $u_{r}(r, t)$ and $u_{\theta}(r, t)$ :

$$
(r, t) \mapsto\left(r, t+d \cdot \tilde{r} / v_{b}\right)
$$

with $\tilde{r}=\left(r-r_{i}\right) / d$, the gap width $d$ and the plume velocity $v_{b}$. For a certain amount of shearing, the plumes which were represented by diagonal lines, become vertical lines. To find this optimal value of shearing, we postulate that when averaging the sheared function over $\tilde{r}$, the standard deviation of the resulting time signal has a maximum as a function of the shearing value. At this optimal shearing, the plumes are represented by narrow peaks in the time signal, creating a high standard deviation. Using a golden section search algorithm [157] the optimal shearing value is found, which corresponds to the mean velocity of the plumes $v_{b}$.

The dependence of the plume velocity on the axial position and the rotation ratio is shown in Fig. 7.7. Again, for inner cylinder rotation only $(a=0)$, no height dependence can be seen. The fluctuations on the radial velocity are moving outwards with a velocity $v_{b} /\left(u_{i}-u_{o}\right)=0.25 \pm 0.02\left(u_{o}=0,2 \sigma\right.$ error $)$. When the azimuthal velocity is considered, the picture looks different. Crossing diagonal lines can be seen, meaning that plumes emit from both the inner and outer cylinder. Despite of this, a maximum was found for inward plumes with an average relative velocity of $v_{b} /\left(u_{i}-u_{o}\right)=0.20 \pm 0.02$. The velocity of these plumes is of the same order, but it remains to be explained why the main plume direction is different for the two velocity components. 

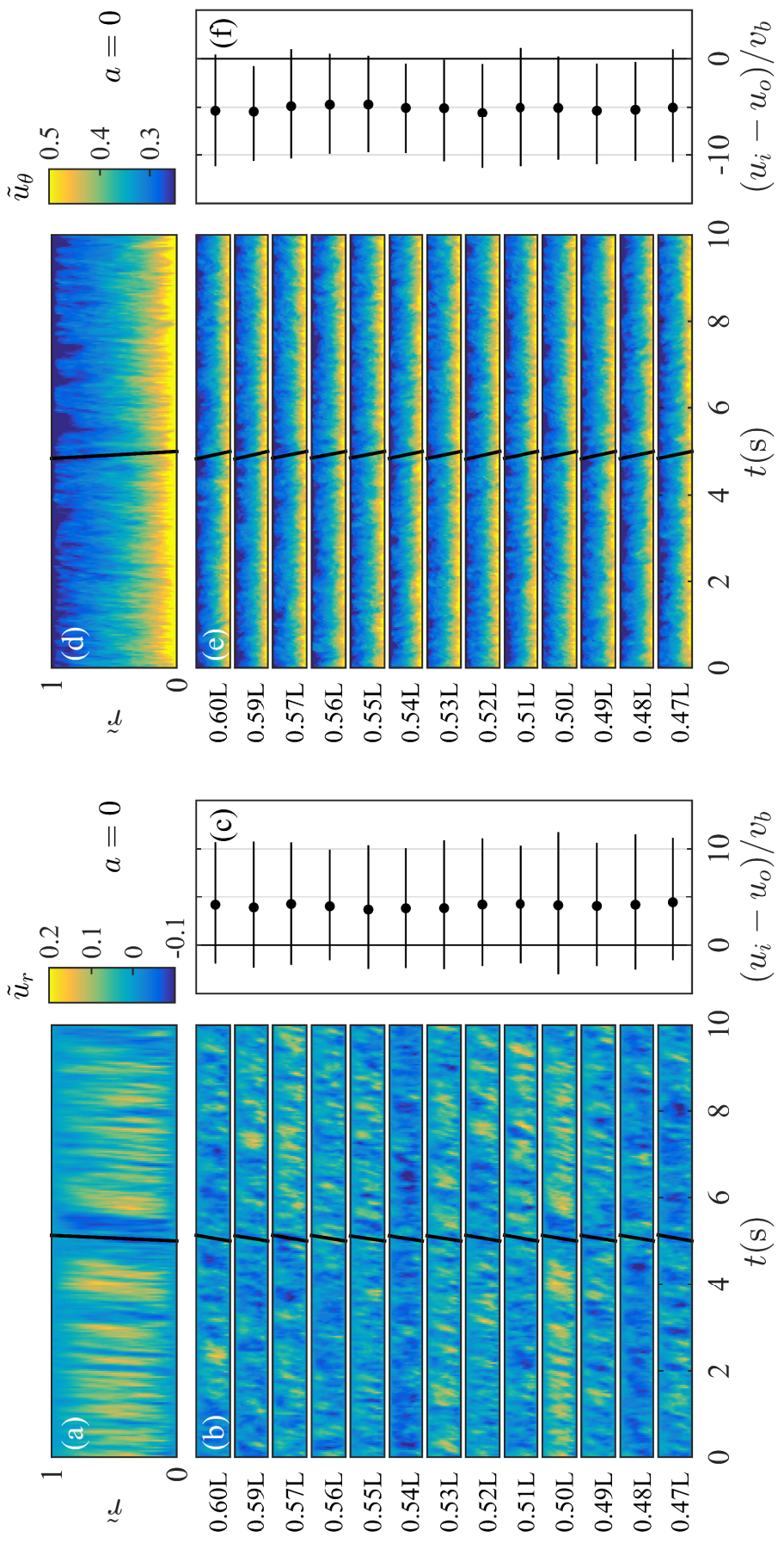


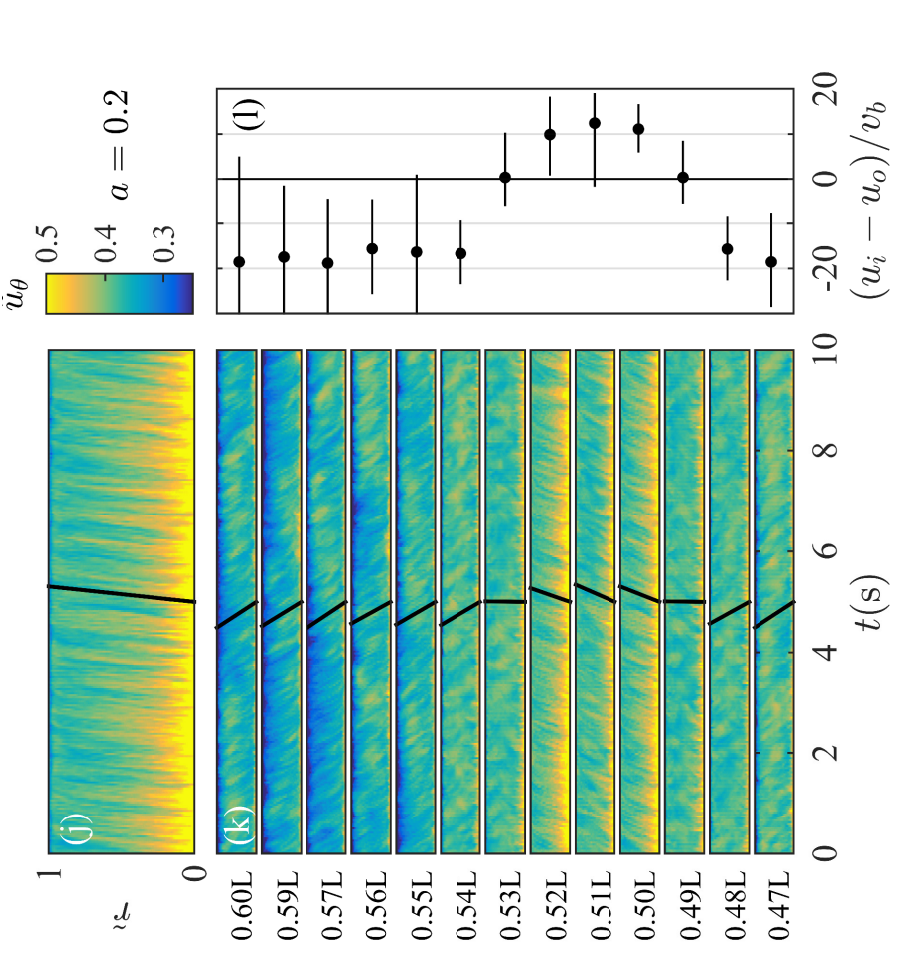

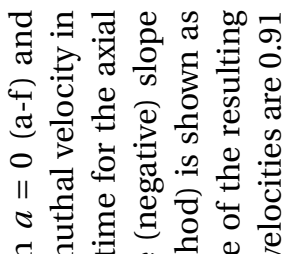

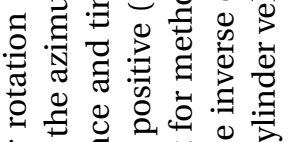

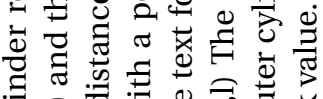

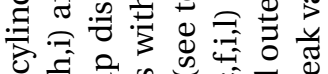

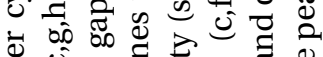
记 ఏ

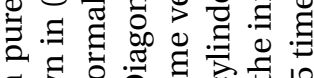

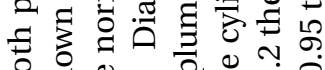

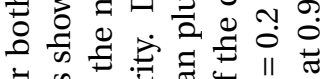
రํ.

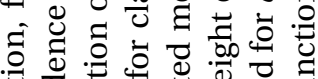
:

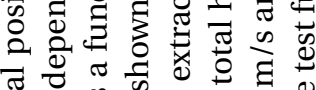

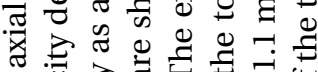

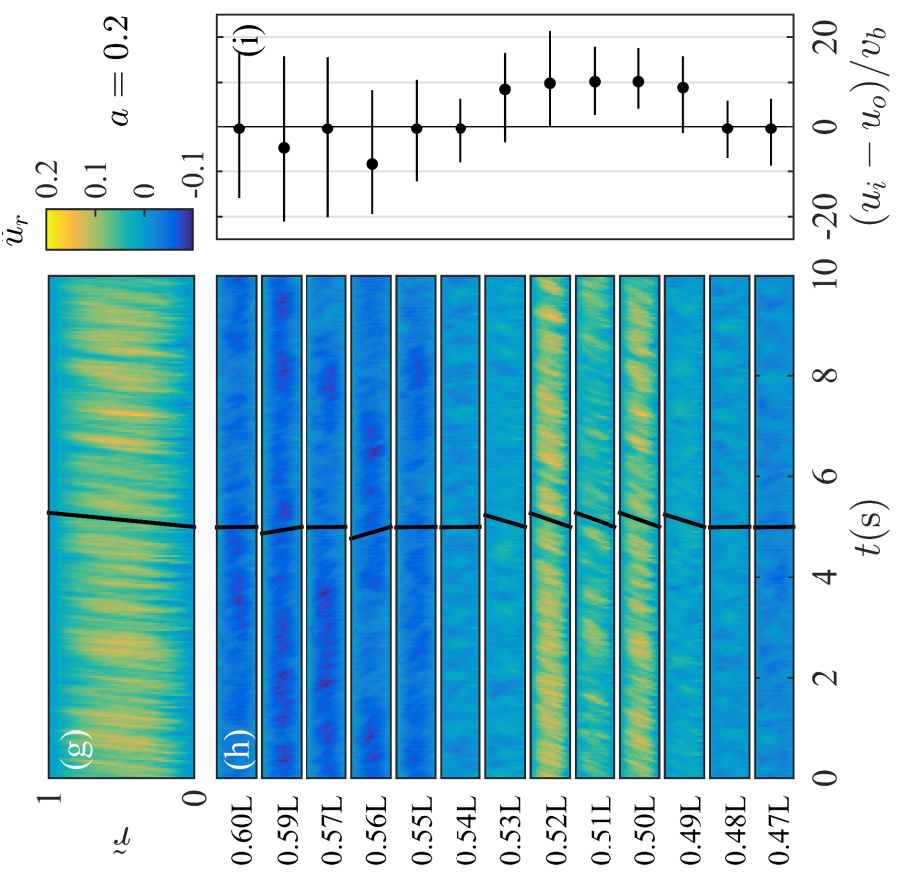

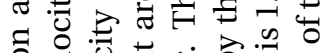

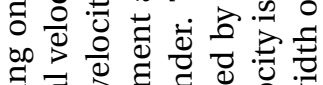
: 记苛 웜 豆

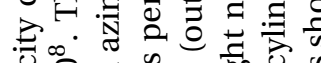
:

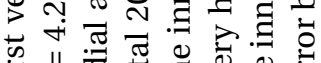

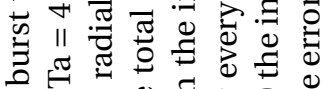
䒕导导

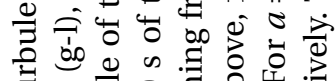

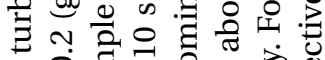

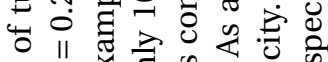

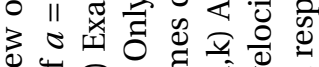
.

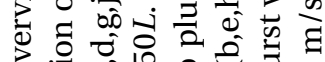

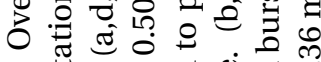

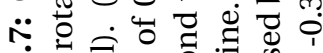

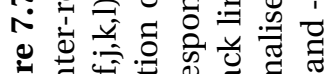

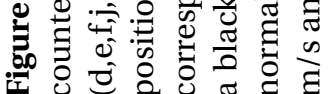


For counter-rotation ( $a=0.2)$, the bottom half of Fig. 7.7 clearly indicates that roll structures are present. There is a correspondence between the sign of the mean radial velocity and the direction of these turbulent plumes, coming from either the inner or outer cylinder. Compare Fig. 7.6(e) with Fig. 7.7(h,k). For both velocity components, the direction of the plumes corresponds to the direction of the mean radial velocity. The mean radial velocity forces the plumes strongly in the inward or outward direction, also typically creating a higher plume velocity than for the $a=0$ case. Whereas for the $a=0$ case there was some ambiguity in the direction of the plumes for the azimuthal velocity, for $a=0.2$ it exactly follows the roll structure. When looking at the radial velocity, the inward plumes are less pronounced than the outward ones, likely due to a tendency for the $u_{r}$ plumes to flow outwards, as could be seen for the $a=0$ case.

In addition to the dependence on the axial position and the rotation ratio, in Fig. 7.8 the dependence of the plume velocity on the driving parameter Ta is shown. With increasing driving strength, the velocity of the plumes also increases. When the plume velocity is normalized by $\left(u_{i}-u_{o}\right)$ it is however nearly constant over two decades of Ta.
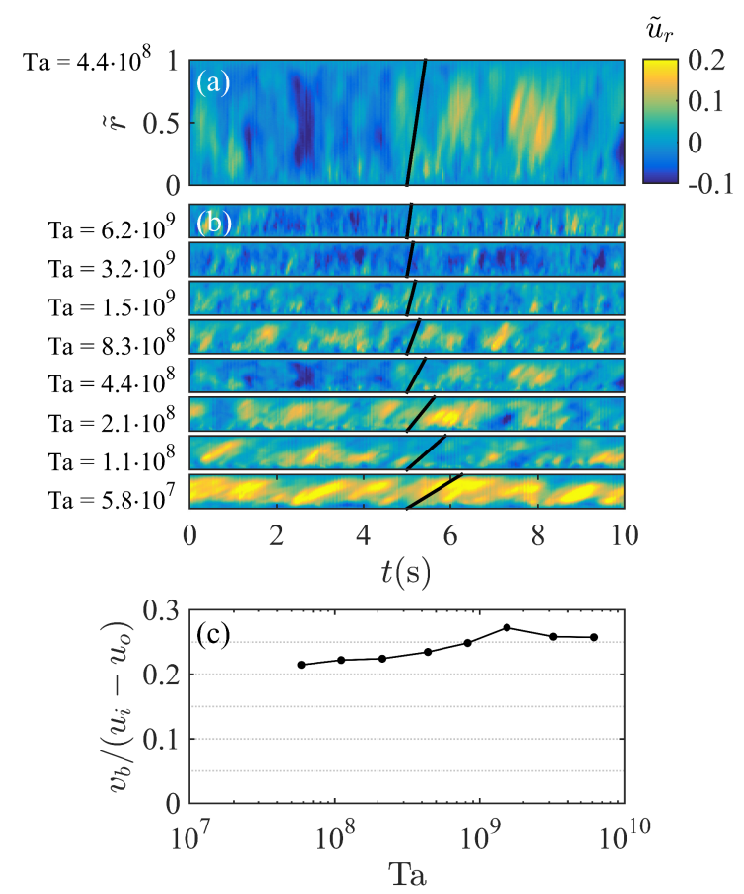

Figure 7.8: Turbulent plumes in the radial velocity for varying Ta and $a=0$. (a) and (b) as in Fig. 7.7. (c) The plume velocity normalized by the inner cylinder velocity $\left(u_{o}=0\right)$. Errors are of the same order as in Fig. 7.7(c). 


\subsubsection{Logarithmic velocity profiles}

It has been proposed that the logarithmic temperature profiles in Rayleigh-Bénard convection and logarithmic velocity profiles in Taylor-Couette flow are triggered by plume ejection [124, 153, 154]. These Refs. also support the notion that parts of the boundary layer can be turbulent, while others are not, and that the transition to the ultimate regime entails plume emission from the full extent of the boundary layer.

We will now further investigate the effect of the plumes on the logarithmic nature of the velocity profiles. As we have seen before, the roll structure that appears for counter-rotation of $a=0.2$ provides strong mean radial flows which cause plume emission away from either the inner or outer cylinder.

In Fig. 7.9(a) the angular velocity at the inner cylinder is shown. It can be seen that there is a significant variation in the shape of the profiles, and that profiles at the axial positions where a strong positive radial flow exists (top lines in Fig. 7.9(c)) follow the shape of a logaritmic law more closely. This effect is even stronger at the outer cylinder in Fig. 7.9(b), where profiles at an axial position with negative radial flow, so a flow away from the outer cylinder, correspond best to a log-law.

To quantify this phenomenom, the velocity profiles are fitted with the modified [152] Prandtl-von Kármán law of the wall: $\omega^{+}=1 / \kappa_{\omega} \ln y^{+}+B$, with both $\kappa_{\omega}$ and $B$ as fitting parameters. We quantify the deviation from a log profile by the standard error of the fitting parameter $\sigma\left(\kappa_{\omega}\right)$. A small value of $\sigma\left(\kappa_{\omega}\right)$ corresponds to a profile which closely follows a log-law. In Fig. 7.9(d) it can be seen that for a mean wall normal velocity away from the cylinder (positive $\langle\tilde{\mathbf{u}} \cdot \mathbf{n}\rangle$ ), the value of $\kappa_{\omega}$ more closely approaches the known classical value of 0.40 . Additionally, the error of the fit is much smaller for positive values of the outward wall normal velocity as compared to negative values. We can summarize that for regions with strong radial flow away from a cylinder surface, plumes will emit from that cylinder, which in turn create logarithmic boundary layers. This is in line with results from direct numerical simulations $[124,154]$. 

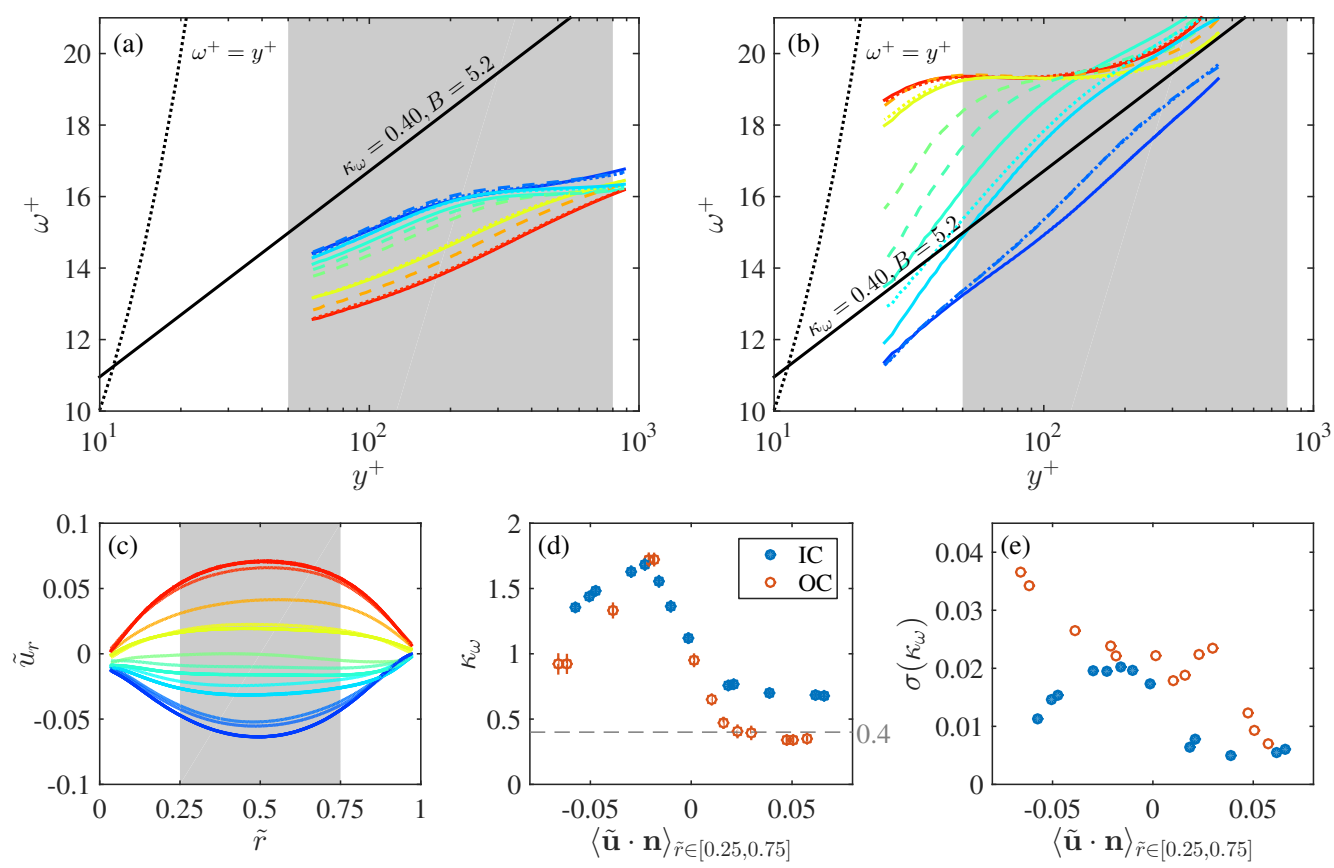

Figure 7.9: Angular velocity profile dependence on mean radial flow for counter-rotation $a=0.2$ and $\mathrm{Ta}=4.2 \cdot 10^{9}$ at varying axial positions (data from Fig. 7.6(h)). (a) Angular velocity profiles near the inner cylinder $(\tilde{r} \in[0,1 / 2])$. The angular velocity $\omega^{+}$and wall normal distance $y^{+}$are defined in the caption of Fig. 7.4, the colors and line styles of the profiles correspond to those in Fig. 7.6. The gray area is the fitting domain $y^{+} \in[50,800]$, chosen to encompass the start of the outer region up to mid-gap for both inner and outer cylinder. (b) Angular velocity profiles near the outer cylinder $(\tilde{r} \in[1 / 2,1])$. (c) normalized radial velocity $\tilde{u}_{r}$ as in Fig. 7.6(f). (d) The von Kármán constant $\kappa_{\omega}$ found by fitting $\omega^{+}=$ $1 / \kappa_{\omega} \ln y^{+}+B$ for $y^{+} \in[50,800]$ near the inner (IC) and outer cylinder (OC). The horizontal axis shows the normalized wall normal velocity, equal to $\tilde{u}_{r}$ and $-\tilde{u}_{r}$ for the IC and OC respectively, averaged over $\tilde{r} \in[1 / 4,3 / 4]$. The error bars have a total length of four times the standard error of the fit. (e) The standard error of the fitting parameter $\kappa_{\omega}$. 


\subsection{Conclusions}

In this chapter we measured velocity profiles, the wind Reynolds number and characteristics of turbulent plumes in Taylor-Couette flow for a radius ratio of 0.5 and Taylor number of up to $6.2 \cdot 10^{9}$. The flow profiles show Taylor vortices for a Taylor number smaller than $\mathrm{Ta} \approx 10^{9}$. The normalized profiles approach the Prandtl-von Kármán log-law, although at the highest $\mathrm{Ta}=6.2 \cdot 10^{9}$ the log layer is not yet fully developed. They are in good agreement with DNS data from other work [88, 109, 151]. Due to the strong curvature of this $\eta=0.5$ setup, a large difference between the azimuthal velocity $u^{+}$and the angular velocity $\omega^{+}$arises. The angular velocity $\omega^{+}$resembles a log-law more closely, as suggested by Grossmann et al. [152], based on their Navier-Stokes based theoretical considerations.

Because of the late onset of the ultimate regime for $\eta=0.5$, the measurements with Ta up to $6.2 \cdot 10^{9}$ are in the classical turbulent regime. For the first time the wind Reynolds number has been measured in the classical regime of TaylorCouette flow and it indeed follows the theoretically predicted classical scaling of $\mathrm{Re}_{\mathrm{w}} \propto \mathrm{Ta}^{3 / 7}$.

Moreover, we focused on the interplay between rolls, turbulent plumes and logarithmic velocity profiles. At a strong driving of $\mathrm{Ta}=4.2 \cdot 10^{9}$ no significant coherent structures exists for pure inner cylinder rotation, but roll structures appear for counter-rotation at $a=-\omega_{o} / \omega_{i}=0.2$. This behavior has been observed previously for different values of $\eta$ and Ta, and shows the important part that rolls play in momentum transfer between the two cylinders.

For inner cylinder rotation only, strong outward plumes are visible in the radial velocity. In the azimuthal velocity, the plumes mainly go inwards. The exact mechanism causing this difference is yet to be elucidated. For counter-rotation, the roll structures strongly influence the direction of the plumes. There is a direct correspondence between the direction of the mean radial flow and the direction of the plumes at a certain position. The plume velocity in the radial flow profiles increases with Taylor number, and has an approximately constant value of one quarter of the inner cylinder velocity for pure inner cylinder rotation.

Lastly, by quantifying the correspondence of the angular velocity profiles to a log-law for several axial positions, it was found that in regions with strong radial flow away from a cylinder surface, plumes will emit from that cylinder, which in turn create logarithmic boundary layers.

This chapter confirms predictions about velocity profiles and the scaling of the wind Reynolds number and sheds new light on the characteristics and the role of plumes in Taylor-Couette flow for a radius ratio $\eta=0.5$, and hopefully will spark further research into these intricate phenomena. 


\section{Conclusions}

In this thesis we set out to answer several questions in the fields of drop impact and Taylor-Couette turbulence. The major results of each chapter are discussed below.

In chapter 1 a high-speed color interferometry method is developed to measure the complete air layer profile and its evolution under an impacting drop. With this method, it is observed that wetting starts at the rim, after which the air under the dimple is entrapped in a bubble. Additionally, the velocity of air flow between the drop and the surface, as well as the velocity of the dimple before wetting occurs, is quantified. These results offer a benchmark for theories of drop impact.

In chapter 2 it is shown that two competing effects minimize the (relative) size of entrained air bubbles: for large drop impact velocity and large drops the inertia of the liquid flattens the entrained bubble, whereas for small impact velocity and small droplets capillary forces minimize the entrained bubble. In between there is an optimum, leading to maximal air bubble entrapment. For a $1.8 \mathrm{~mm}$ diameter ethanol droplet this optimum is achieved at an impact velocity of $0.25 \mathrm{~m} / \mathrm{s}$. These findings can be applied to various industrial and technical applications to control the size of entrained bubbles.

In chapter 3 it is shown that for drop impact on micropatterned surfaces, the liquid-air interface globally resembles the azimuthally symmetric dimple on smooth surfaces, however with local symmetry-breaking deformation caused by the microstructure. It was found that the central dimple height is increased due to presence of protruding pillars, which create an additional pressure build-up. However, in the case of a sparse array of micropillars, the maximum dimple height is hardly influenced by the height of the pillars. These results and the employed imaging technique can facilitate the understanding of drop impact on rough surfaces.

In chapter 4 the design and construction of a new Taylor-Couette system with independently rotating cylinders and precise temperature control is treated. The performance of the engines and angular encoders are tested and show very good performance for rotation rates ranging from $0.1 \mathrm{~Hz}$ to $20 \mathrm{~Hz}$. The setup is able to cool and heat the outer and inner cylinder independently and was found to have a very good temperature stability. The good optical accessibility is shown by performing PIV measurements from the top, and imaging turbulent coherent struc- 
tures in the vertical plane of the apparatus using a high-resolution camera. The new facility offers the possibility to accurately study the process of boiling inside a turbulent flow and its effect on the flow. Many open questions on the boiling process remain and possible future studies include studying the nucleation process in this well-controlled turbulent environment, and the clustering dynamics of vapor clouds and its effects on nucleation.

In chapter 5 it is shown that Taylor-Couette flow displays flow structure even for a very high Taylor number of roughly $10^{12}\left(\mathrm{Re}=\mathscr{O}\left(10^{6}\right)\right)$. In addition, it is found that the system is hysteretic and can be in multiple stable turbulent states for the same driving parameters. It is found that in this setup, multiple states can occur for rotation ratios $0.17<a<0.51$. For $0 \leq a<0.17$ there is only a single stable state, although it cannot be excluded that other trajectories in phase space might trigger multiple states in this region. For $a>0.51$ the system does not possess a state with a clear roll structure. This chapter highlights the importance of the coherent structures and their selectability in highly turbulent flows. The question of whether or not these structures survive for even larger Reynolds numbers, is important for understanding the myriad of large-scale flows in nature, and is treated in chapter 6 .

In chapter 6 it is found that multiple states in highly turbulent Taylor-Couette flow are very robust. Their existence was observed for a Taylor number range of almost two decades, and it can be expected that they persist for $\mathrm{Ta}=10^{13}$ and beyond. Although their existence is robust, to reliably reach the less-likely low torque state in our $\mathrm{T}^{3} \mathrm{C}$ setup, complex paths in phase space can be necessary. The rotation ratio $a$ is the parameter that most strongly controls the transitions between the flow states, and the transitional values only weakly depend on the Taylor number. The robustness of the multiple states also shows in the measurements that were performed in a different setup with a larger aspect ratio. Here, the phenomena are even more rich, although the same principles hold; the system is hysteretic and can be in multiple stable turbulent states for the same driving parameters. For the larger aspect ratio, the system can be in a larger number of different roll states. Additionally, for the first time in turbulent TC flow, an antisymmetrical roll state has been observed. Potential directions for further study are: improving the understanding of the transitional $a$-values and the probabilistic nature of the bifurcations.

In chapter 7 velocity profiles, the wind Reynolds number and characteristics of turbulent plumes are investigated in Taylor-Couette flow for a radius ratio of 0.5 and Taylor number of up to $6.2 \cdot 10^{9}$. The extracted angular velocity profiles follow a log-law more closely than the azimuthal velocity profiles due to the strong curvature of this $\eta=0.5$ setup. The scaling of the wind Reynolds number with the Taylor number agrees with the theoretically predicted $3 / 7$-scaling for the classical turbulent regime, which is much more pronounced than for the well-explored 
$\eta=0.71$ case, for which the ultimate regime sets in at much lower Ta. In addition, turbulent plumes coming from the inner and outer cylinder are investigated. For pure inner cylinder rotation, the plumes in the radial velocity move away from the inner cylinder, while the plumes in the azimuthal velocity mainly move away from the outer cylinder. For counter-rotation, the mean radial flow in the roll structures strongly affects the direction and intensity of the turbulent plumes. Furthermore, it is experimentally confirmed that in regions where plumes are emitted, boundary layer profiles with a logarithmic signature are created. This chapter confirms predictions about velocity profiles and the scaling of the wind Reynolds number and sheds new light on the characteristics and the role of plumes in Taylor-Couette flow for a radius ratio $\eta=0.5$. 


\section{Bibliography}

[1] A.M. Worthington, A study of splashes (Longmans, Green, and Co.) (1908).

[2] M. Rein, "Phenomena of liquid drop impact on solid and liquid surfaces", Fluid Dyn. Res. 12, 61 (1993).

[3] A. L. Yarin, “Drop impact dynamics: Splashing, spreading, receding, bouncing...”, Annu. Rev. Fluid Mech. 38, 159-192 (2006).

[4] C. Josserand and S. Thoroddsen, "Drop impact on a solid surface”, Annu. Rev. Fluid Mech. 48 (2016).

[5] S. Thoroddsen, T. Etoh, and K. Takehara, "High-speed imaging of drops and bubbles”, Annu. Rev. Fluid Mech. 40, 257-285 (2008).

[6] M. Versluis, “High-speed imaging in fluids", Exp. Fluids 54 (2013).

[7] M. Couette, “Études sur le frottement des liquides”, Ann. Chim. Phys. 21, 433 (1890).

[8] A. Mallock, "Experiments on Fluid Viscosity”, Phil. Trans. R. Soc. Lond. A 187, 41-56 (1896).

[9] G. I. Taylor, "Stability of a viscous liquid contained between two rotating cylinders", Phil. Trans. R. Soc. A 223, 289-343 (1923).

[10] C. D. Andereck, S. S. Liu, and H. L. Swinney, "Flow regimes in a circular Couette system with independently rotating cylinders”, J. Fluid Mech. 164, 155-183 (1986).

[11] S. Grossmann, D. Lohse, and C. Sun, "High Reynolds number Taylor-Couette turbulence”, Annu. Rev. Fluid Mech. 48, 53-80 (2016).

[12] B. Eckhardt, S. Grossmann, and D. Lohse, “Torque scaling in turbulent TaylorCouette flow between independently rotating cylinders”, J. Fluid Mech. 581, 221250 (2007).

[13] J. Westerweel, G. E. Elsinga, and R. J. Adrian, "Particle image velocimetry for complex and turbulent flows”, Annu. Rev. Fluid Mech. 45, 409-436 (2013).

[14] S. Grossmann and D. Lohse, "Scaling in thermal convection: A unifying view", J. Fluid Mech. 407, 27-56 (2000). 
[15] A.M. Worthington, "On the forms assumed by drops of liquids falling vertically on a horizontal plate”, Proc. Royal Soc. London 25, 261 (1876).

[16] L. Xu, W.W. Zhang and S.R. Nagel, “Drop splashing on a dry smooth surface”, Phys. Rev. Lett. 94, 184505 (2005).

[17] M.M. Driscoll and S.R. Nagel, "Ultrafast interference imaging of air in splashing dynamics”, Phys. Rev. Lett. 107, 154502 (2011).

[18] S. Thoroddsen, M. Thoraval, K. Takehara, and T. Etoh, "Droplet splashing by a slingshot mechanism”, Phys. Rev. Lett. 106, 34501 (2011).

[19] S. Mandre, M. Mani and M.P. Brenner, "Precursors to splashing of liquid droplets on a solid surface”, Phys. Rev. Lett. 102, 134502 (2009).

[20] M. Mani, S. Mandre and M.P. Brenner, "Events before droplet splashing on a solid surface”, J. Fluid Mech. 647, 163-185 (2010).

[21] P.D. Hicks and R. Purvis, "Air cushioning and bubble entrapment in threedimensional droplet impacts”, J. Fluid Mech. 649, 135-163 (2010).

[22] S. Mandre and M.P. Brenner, "The mechanism of a splash on a dry solid surface”, J. Fluid Mech. 690, 148-172 (2012).

[23] S. Chandra and C.T. Avedisian, "On the collision of a droplet with a solid surface", Proc. Royal Soc. London, A 432, 13-41 (1991).

[24] S.T. Thoroddsen and J. Sakakibara, "Evolution of the fingering pattern of an impacting drop”, Phys. Fluids 10, 1359 (1998).

[25] D.B. van Dam and C. Le Clerc, "Experimental study of the impact of an ink-jet printed droplet on a solid substrate”, Phys. Fluids 16, 3403 (2004).

[26] S. Thoroddsen, T. Etoh, K. Takehara, N. Ootsuka, and A. Hatsuki, “The air bubble entrapped under a drop impacting on a solid surface”, J. Fluid Mech. 545, 203-212 (2005).

[27] R.W.G. Hunt, Measuring Colour (Fountain Press, Kingston-Upon-Thames, UK) (1998).

[28] C. Lin and R.F. Sullivan, "An application of white light interferometry in thin film measurements”, IBM J. Research and Development 16, 269-276 (1972).

[29] J. M. Kolinski, S. M. Rubinstein, S. Mandre, M. Mani, M. P. Brenner, D. A. Weitz, and L. Mahadevan, "Skating on a film of air: Drops impacting on a surface", Phys. Rev. Lett. 108, 074503 (2012).

[30] L. Xu, L. Barcos, and S. R. Nagel, "Splashing of liquids: Interplay of surface roughness with surrounding gas”, Phys. Rev. E 76, 066311 (2007). 
[31] M. H. W. Hendrix, R. Manica, E. Klaseboer, D. Y. C. Chan, and C.-D. Ohl, "Spatiotemporal evolution of thin liquid films during impact of water bubbles on glass on a micrometer to nanometer scale”, Phys. Rev. Lett. 108, 247803 (2012).

[32] P. D. Hicks and R. Purvis, "Air cushioning in droplet impacts with liquid layers and other droplets", Phys. Fluids 23, 062104 (2011).

[33] J. Eggers, M. A. Fontelos, C. Josserand, and S. Zaleski, "Drop dynamics after impact on a solid wall: Theory and simulations”, Phys. Fluids 22, 062101 (2010).

[34] H. N. Oguz and A. Prosperetti, "Dynamics of bubble-growth and detachment from a needle.", J. Fluid Mech. 257, 111-145 (1993).

[35] R. P. H. M. Bergmann, D. van der Meer, S. Gekle, J. van der Bos, and D. Lohse, "Controlled impact of a disk on a water surface: cavity dynamics”, J. Fluid Mech. 633, 381-409 (2009).

[36] S. Gekle and J. Gordillo, "Compressible air flow through a collapsing liquid cavity", Int. J. Numer. Meth. Fluids 67 (2011).

[37] F. T. Smith, L. Li, and G. X. Wu, “Air cushioning with a lubrication/inviscid balance", J. Fluid Mech. 482, 291-318 (2003).

[38] M. Switkes, M. Rothschild, T. A. Shedd, H. B. Burnett, and M. S. Yeung, "Bubbles in immersion lithography”, J. Vac. Sci. Technol. B 23, 2409-2412 (2005).

[39] K. G. Winkels, I. R. Peters, F. Evangelista, M. Riepen, A. Daerr, L. Limat, and J. H. Snoeijer, "Receding contact lines: From sliding drops to immersion lithography", EPJ - Special Topics 192, 195-205 (2011).

[40] J. S. Lee, B. M. Weon, J. H. Je, and K. Fezzaa, "How does an air film evolve into a bubble during drop impact?”, Phys. Rev. Lett. 109, 204501 (2012).

[41] M. Reyssat, D. Richard, C. Clanet, and D. Quéré, "Dynamical superhydrophobicity”, Faraday Discuss. 146, 19-33 (2010).

[42] P. Tsai, R. C. A. Van Der Veen, M. Van De Raa, and D. Lohse, "How micropatterns and air pressure affect splashing on surfaces”, Langmuir 26, 16090-16095 (2010).

[43] P. Tsai, M. H. W. Hendrix, R. R. M. Dijkstra, L. Shui, and D. Lohse, "Microscopic structure influencing macroscopic splash at high weber number", Soft Matter 7, 1132511333 (2011).

[44] L. Courbin, E. Denieul, E. Dressaire, M. Roper, A. Ajdari, and H. A. Stone, "Imbibition by polygonal spreading on microdecorated surfaces”, Nat. Mater. 6, 661-664 (2007).

[45] S. Moulinet and D. Bartolo, "Life and death of a fakir droplet: Impalement transitions on superhydrophobic surfaces”, Eur. Phys. J. E 24, 251-260 (2007). 
[46] M. Sbragaglia, A. M. Peters, C. Pirat, B. M. Borkent, R. G. H. Lammertink, M. Wessling, and D. Lohse, "Spontaneous breakdown of superhydrophobicity", Phys. Rev. Lett. 99, 156001 (2007).

[47] C. Pirat, M. Sbragaglia, A. M. Peters, B. M. Borkent, R. G. H. Lammertink, M. Wessling, and D. Lohse, "Multiple time scale dynamics in the breakdown of superhydrophobicity”, Europhys. Lett. 81, 66002 (2008).

[48] P. Papadopoulos, L. Mammen, X. Deng, D. Vollmer, and H. Butt, "How superhydrophobicity breaks down”, P. Natl. Acad. Sci. USA 1 10, 3254-3258 (2013).

[49] I. U. Vakarelski, N. A. Patankar, J. O. Marston, D. Y. C. Chan, and S. T. Thoroddsen, "Stabilization of leidenfrost vapour layer by textured superhydrophobic surfaces", Nature 489, 274-277 (2012).

[50] D. Quéré, “Leidenfrost dynamics”, Annu. Rev. Fluid Mech. 45, 197-215 (2013).

[51] H. Nair, H. J. J. Staat, T. Tran, A. van Houselt, A. Prosperetti, D. Lohse, and C. Sun, "The leidenfrost temperature increase for impacting droplets on carbon-nanofiber surfaces", Soft Matter 10, 2102-2109 (2014).

[52] T. Tran, H. J. J. Staat, A. Susarrey-Arce, T. C. Foertsch, A. Van Houselt, H. J. G. E. Gardeniers, A. Prosperetti, D. Lohse, and C. Sun, "Droplet impact on superheated micro-structured surfaces”, Soft Matter 9, 3272-3282 (2013).

[53] H. V. Jansen, J. G. E. Gardeniers, J. Elders, H. A. C. Tilmans, and M. Elwenspoek, "Applications of fluorocarbon polymers in micromechanics and micromachining", Sensor. Actuat. A-Phys. 41, 136-140 (1994).

[54] R. N. Wenzel, "Resistance of solid surfaces to wetting by water", Ind. Eng. Chem. 28, 988-994 (1936).

[55] A. B. D. Cassie and S. Baxter, "Wettability of porous surfaces", T. Faraday Soc. 40, 546-551 (1944).

[56] J. Bico, C. Marzolin, and D. Quéré, “Pearl drops”, Europhys. Lett. 47, 220-226 (1999).

[57] D. Quéré, “Surface chemistry: Fakir droplets”, Nat. Mater. 1, 14-15 (2002).

[58] L. Feng, S. H. Li, Y. S. Li, H. J. Li, L. J. Zhang, J. Zhai, Y. L. Song, B. Q. Liu, L. Jiang, and D. B. Zhu, "Super-hydrophobic surfaces: From natural to artificial”, Adv. Mater. 14, 1857-1860 (2002).

[59] P. R. Fenstermacher, H. L. Swinney, and J. P. Gollub, "Dynamical instabilities and the transition to chaotic Taylor vortex flow”, J. Fluid Mech. 94, 103-128 (1979).

[60] R. C. Di Prima and H. L. Swinney, "Instabilities and transition in flow between concentric rotating cylinders", in Hydrodynamic Instabilities and the Transition to Turbulence, edited by H. L. Swinney and J. P. Gollub, volume 45 of Topics in Applied Physics, 139-180 (Springer Berlin Heidelberg) (1985). 
[61] R. C. Aldredge, "A novel flow reactor for the study of heat-loss effects on turbulent flame propagation”, Int. Commun. Heat Mass 23, 1173 - 1179 (1996).

[62] R. Aldredge, V. Vaezi, and P. Ronney, "Premixed-flame propagation in turbulent Taylor-Couette flow”, Combust. Flame 115, 395 - 405 (1998).

[63] P. D. Ronney, B. D. Haslam, and N. O. Rhys, "Front propagation rates in randomly stirred media”, Phys. Rev. Lett. 74, 3804-3807 (1995).

[64] T. H. van den Berg, S. Luther, D. P. Lathrop, and D. Lohse, "Drag reduction in bubbly Taylor-Couette turbulence”, Phys. Rev. Lett. 94, 044501 (2005).

[65] K. Sugiyama, E. Calzavarini, and D. Lohse, "Microbubbly drag reduction in TaylorCouette flow in the wavy vortex regime”, J. Fluid Mech. 608, 21-41 (2008).

[66] D. P. M. van Gils, D. Narezo-Guzman, C. Sun, and D. Lohse, “The importance of bubble deformability for strong drag reduction in bubbly turbulent Taylor-Couette flow", J. Fluid Mech. 722, 317-347 (2013).

[67] S. Srinivasan, J. A. Kleingartner, J. B. Gilbert, R. E. Cohen, A. J. Milne, and G. H. McKinley, "Sustainable drag reduction in turbulent taylor-couette flows by depositing sprayable superhydrophobic surfaces”, Phys. Rev. Lett. 114, 014501 (2015).

[68] S. Chandrasekhar, Hydrodynamic and hydromagnetic stability, volume 196 (Courier Corporation) (1961).

[69] S. A. Balbus and J. F. Hawley, "A powerful local shear instability in weakly magnetized disks. I - Linear analysis. II - Nonlinear evolution”, Astrophys. J. 376, 214-233 (1991).

[70] G. Rüdiger and Y. Zhang, "MHD instability in differentially-rotating cylindric flows", A\&A 378, 302-308 (2001).

[71] H. Ji, J. Goodman, and A. Kageyama, "Magnetorotational instability in a rotating liquid metal annulus”, Mon. Not. R. Astron. Soc. 325, L1-L5 (2001).

[72] R. Hollerbach, V. Teeluck, and G. Rüdiger, "Nonaxisymmetric magnetorotational instabilities in cylindrical Taylor-Couette flow”, Phys. Rev. Lett. 104, 044502 (2010).

[73] D. Richard and J. Zahn, “Turbulence in differentially rotating flows what can be learned from the Couette-Taylor experiment”, A\&A 347, 732 (1999).

[74] B. Dubrulle, O. Dauchot, F. Daviaud, P. Y. Longgaretti, D. Richard, and J. P. Zahn, "Stability and turbulent transport in Taylor-Couette flow from analysis of experimental data", Phys. Fluids 17, 095103 (2005).

[75] E. Schartman, H. Ji, M. J. Burin, and J. Goodman, "Stability of quasi-Keplerian shear flow in a laboratory experiment”, A\& A 543, A94 (2012). 
[76] M. S. Paoletti, D. P. M. van Gils, B. Dubrulle, C. Sun, D. Lohse, and D. P. Lathrop, "Angular momentum transport and turbulence in laboratory models of Keplerian flows”, A\&A 547, A64 (2012).

[77] K.-I. Ohashi, K. Tashiro, F. Kushiya, T. Matsumoto, S. Yoshida, M. Endo, T. Horio, K. Ozawa, and K. Sakai, "Rotation-induced Taylor vortex enhances filtrate flux in plasma separation”, ASAIO Journal 34, 300-307 (1988).

[78] G. Beaudoin and M. Y. Jaffrin, "Plasma filtration in couette flow membrane devices", Artificial Organs 13, 43-51 (1989).

[79] G. A. Ameer, S. Raghavan, R. Sasisekharan, W. Harmon, C. L. Cooney, and R. Langer, "Regional heparinization via simultaneous separation and reaction in a novel Taylor-Couette flow device”, Biotechnol. Bioeng. 63, 618-624 (1999).

[80] S. T. Wereley and R. M. Lueptow, "Inertial particle motion in a Taylor-Couette rotating filter”, Phys. Fluids 11, 325-333 (1999).

[81] E. Serre, M. A. Sprague, and R. M. Lueptow, "Stability of Taylor-Couette flow in a finite-length cavity with radial throughflow”, Phys. Fluids 20, 034106 (2008).

[82] T.-M. Jeng, S.-C. Tzeng, and C.-H. Lin, "Heat transfer enhancement of TaylorCouette-Poiseuille flow in an annulus by mounting longitudinal ribs on the rotating inner cylinder", Int. J. Heat Mass. Tran. 50, 381-390 (2007).

[83] G. S. Lewis and H. L. Swinney, "Velocity structure functions, scaling, and transitions in high-Reynolds-number Couette-Taylor flow”, Phys. Rev. E 59, 5457-5467 (1999).

[84] D. P. M. van Gils, S. G. Huisman, G. W. Bruggert, C. Sun, and D. Lohse, “Torque scaling in turbulent Taylor-Couette flow with co- and counter-rotating cylinders”, Phys. Rev. Lett. 106, 024502 (2011).

[85] M. S. Paoletti and D. P. Lathrop, “Angular momentum transport in turbulent flow between independently rotating cylinders”, Phys. Rev. Lett. 106, 024501 (2011).

[86] S. G. Huisman, D. Lohse, and C. Sun, "Statistics of turbulent fluctuations in counterrotating Taylor-Couette flows”, Phys. Rev. E 88, 063001 (2013).

[87] S. G. Huisman, S. Scharnowski, C. Cierpka, C. Kähler, D. Lohse, and C. Sun, "Logarithmic boundary layers in strong Taylor-Couette turbulence”, Phys. Rev. Lett. 110, 264501 (2013).

[88] R. Ostilla-Mónico, E. P. van der Poel, R. Verzicco, S. Grossmann, and D. Lohse, “Exploring the phase diagram of fully turbulent Taylor-Couette flow”, J. Fluid Mech. 761, 1-26 (2014).

[89] R. Ostilla-Mónico, R. Verzicco, S. Grossmann, and D. Lohse, “Turbulence decay towards the linearly stable regime of Taylor-Couette flow”, J. Fluid Mech. 748, R3 (2014). 
[90] R. Ostilla-Mónico, S. G. Huisman, T. J. G. Jannink, D. P. M. Van Gils, R. Verzicco, S. Grossmann, C. Sun, and D. Lohse, "Optimal Taylor-Couette flow: radius ratio dependence”, J. Fluid Mech. 747, 1-29 (2014).

[91] S. Cohen and M. D. Moalem, "Analysis of a rotating annular reactor in the vortex flow regime”, Chem. Eng. Sci. 46, 123 - 134 (1991).

[92] G. A. Ameer, W. Harmon, R. Sasisekharan, and R. Langer, "Investigation of a whole blood fluidized bed Taylor-Couette flow device for enzymatic heparin neutralization", Biotechnol. Bioeng. 62, 602-608 (1999).

[93] G. A. Ameer, G. Barabino, R. Sasisekharan, W. Harmon, C. L. Cooney, and R. Langer, "Ex vivo evaluation of a Taylor-Couette flow, immobilized heparinase i device for clinical application”, Proc. Nat. Acad. Sci. USA 96, 2350-2355 (1999).

[94] R. Lakkaraju, L. E. Schmidt, P. Oresta, F. Toschi, R. Verzicco, D. Lohse, and A. Prosperetti, "Effect of vapor bubbles on velocity fluctuations and dissipation rates in bubbly rayleigh-bénard convection”, Phys. Rev. E 84, 036312 (2011).

[95] R. Lakkaraju, R. J. A. M. Stevens, P. Oresta, R. Verzicco, D. Lohse, and A. Prosperetti, "Heat transport in bubbling turbulent convection", P. Natl. Acad. Sci. USA 110, 92379242 (2013).

[96] M. A. J. van Limbeek, H. Lhuissier, A. Prosperetti, C. Sun, and D. Lohse, "Explosive boiling?", Phys. Fluids 25, 091102 (2013).

[97] X. Zhang, H. Lhuissier, C. Sun, and D. Lohse, "Surface nanobubbles nucleate microdroplets”, Phys. Rev. Lett. 112, 144503 (2014).

[98] V. K. Dhir, “Boiling heat transfer”, Annu. Rev. Fluid Mech. 30, 365-401 (1998).

[99] T. Theofanous, J. Tu, A. Dinh, and T.-N. Dinh, “The boiling crisis phenomenon: Part I: nucleation and nucleate boiling heat transfer", Exp. Therm. Fluid Sci. 26, 775-792 (2002).

[100] V. K. Dhir, "Mechanistic prediction of nucleate boiling heat transfer-achievable or a hopeless task?”, J. Heat Tran. 128, 1-12 (2006).

[101] J. Kim, “Review of nucleate pool boiling bubble heat transfer mechanisms", Int. J. Multiphas. Flow 35, 1067 - 1076 (2009).

[102] J.-Q. Zhong, D. Funfschilling, and G. Ahlers, "Enhanced heat transport by turbulent two-phase Rayleigh-Bénard convection”, Phys. Rev. Lett. 102, 124501 (2009).

[103] D. P. M. van Gils, G. W. Bruggert, D. P. Lathrop, C. Sun, and D. Lohse, “The Twente turbulent Taylor-Couette $\left(T^{3} C\right.$ ) facility: strongly turbulent (multi-phase) flow between independently rotating cylinders”, Rev. Sci. Instr. 82, 025105 (2011).

[104] M. A. Fardin, C. Perge, and N. Taberlet, ““The hydrogen atom of fluid dynamics" Introduction to the Taylor-Couette flow for Soft Matter scientists", Soft Matter 10, 3523-3535 (2014). 
[105] D. P. Lathrop, J. Fineberg, and H. S. Swinney, “Transition to shear-driven turbulence in Couette-Taylor flow”, Phys. Rev. A 46, 6390-6405 (1992).

[106] E. Schartman, H. Ji, and M. J. Burin, “Development of a Couette-Taylor flow device with active minimization of secondary circulation”, Rev. Sci. Inst. 80, 024501 (2009).

[107] S. Merbold, H. J. Brauckmann, and C. Egbers, “Torque measurements and numerical determination in differentially rotating wide gap Taylor-Couette flow”, Phys. Rev. E 87, 023014 (2013).

[108] K. Avila and B. Hof, "High-precision Taylor-Couette experiment to study subcritical transitions and the role of boundary conditions and size effects", Rev. Sci. Inst. 84, 065106 (2013).

[109] A. Chouippe, E. Climent, D. Legendre, and C. Gabillet, "Numerical simulation of bubble dispersion in turbulent Taylor-Couette flow”, Phys. Fluids 26, 043304 (2014).

[110] S. A. Balbus, "Enhanced angular momentum transport in accretion disks", Annu. Rev. Astron. Astr. 41, 555-597 (2003).

[111] H. Ji and S. Balbus, "Angular momentum transport in astrophysics and in the lab", Phys. Today 66, 27-33 (2013).

[112] A. N. Kolmogorov, “The local structure of turbulence in incompressible viscous fluid for very large Reynolds numbers”, Dokl. Akad. Nauk. SSSR. 30, 299-303 (1941).

[113] A. N. Kolmogorov, "On degeneration of isotropic turbulence in an incompressible viscous liquid”, Dokl. Akad. Nauk. SSSR. 31 (1941).

[114] H.-D. Xi and K.-Q. Xia, "Flow mode transitions in turbulent thermal convection", Phys. Fluids 20, 055104 (2008).

[115] E. P. van der Poel, R. J. A. M. Stevens, and D. Lohse, “Connecting flow structures and heat flux in turbulent Rayleigh-Bénard convection”, Phys. Rev. E 84, 045303 (2011).

[116] S. Weiss and G. Ahlers, "Effect of tilting on turbulent convection: cylindrical samples with aspect ratio $\Gamma=0.50 "$, J. Fluid. Mech. 715, 314-334 (2013).

[117] G. Ahlers, D. Funfschilling, and E. Bodenschatz, "Heat transport in turbulent Rayleigh-Bénard convection for $\operatorname{Pr} \simeq 0.8$ and $\mathrm{Ra} \lesssim 10^{15}$, J. Phys.: Conf. Series 318, 082001 (2011).

[118] S. Grossmann and D. Lohse, "Multiple scaling in the ultimate regime of thermal convection”, Phys. Fluids 23, 045108 (2011).

[119] F. Ravelet, L. Marié, A. Chiffaudel, and F. Daviaud, "Multistability and memory effect in a highly turbulent flow: Experimental evidence for a global bifurcation”, Phys. Rev. Lett. 93, 164501 (2004).

[120] F. Ravelet, A. Chiffaudel, and F. Daviaud, "Supercritical transition to turbulence in an inertially driven von Kámán closed flow”, J. Fluid Mech. 601, 339-364 (2008). 
[121] P.-P. Cortet, A. Chiffaudel, F. Daviaud, and B. Dubrulle, "Experimental evidence of a phase transition in a closed turbulent flow”, Phys. Rev. Lett. 105, 214501 (2010).

[122] D. S. Zimmerman, S. A. Triana, and D. P. Lathrop, "Bi-stability in turbulent, rotating spherical Couette flow”, Phys. Fluids 23, - (2011).

[123] R. Ostilla-Mónico, R. J. A. M. Stevens, S. Grossmann, R. Verzicco, and D. Lohse, “Optimal Taylor-Couette flow: direct numerical simulations”, J. Fluid Mech. 719, 14-46 (2013).

[124] R. Ostilla-Mónico, E. P. van der Poel, R. Verzicco, S. Grossmann, and D. Lohse, "Boundary layer dynamics at the transition between the classical and the ultimate regime of Taylor-Couette flow", Phys. Fluids 26, 015114 (2014).

[125] R. H. Kraichnan, "Turbulent thermal convection at arbritrary Prandtl number", Phys. Fluids 5, 1374-1389 (1962).

[126] F. Ravelet, R. Delfos, and J. Westerweel, "Influence of global rotation and reynolds number on the large-scale features of a turbulent Taylor-Couette flow", Phys. Fluids 22, 055103 (2010).

[127] D.P.M. van Gils, S. G. Huisman, S. Grossmann, C. Sun, and D. Lohse, “Optimal taylorcouette turbulence", J. Fluid Mech. 708, 118 (2012).

[128] S. G. Huisman, D. P. M. van Gils, S. Grossmann, C. Sun, and D. Lohse, "Ultimate turbulent Taylor-Couette flow", Phys. Rev. Lett. 108, 024501 (2012).

[129] H. Brauckmann and B. Eckhardt, "Direct Numerical Simulations of Local and Global Torque in Taylor-Couette Flow up to Re=30.000”, J. Fluid Mech. 718, 398-427 (2013).

[130] S. G. Huisman, D. P. van Gils, and C. Sun, "Applying laser doppler anemometry inside a Taylor-Couette geometry using a ray-tracer to correct for curvature effects", Eur. J. Mech. B-Fluid 36, 115 - 119 (2012).

[131] A. Chouippe, "Etude numérique de la réduction de traînée par injection de bulles en écoulement de Taylor-Couette”, PhD thesis, l'Institute National Polytechnique de Toulouse (INPT) (2012).

[132] M. López-Caballero and J. Burguete, "Inverse cascades sustained by the transfer rate of angular momentum in a 3d turbulent flow”, Phys. Rev. Lett. 110, 124501 (2013).

[133] R. J. Donnelly, “Taylor-Couette flow: the early days”, Phys. Today 44(11), 32-39 (1991).

[134] F. Wendt, “Turbulente Strömungen zwischen zwei rotierenden konaxialen Zylindern”, Ingenieur-Archiv 4, 577-595 (1933).

[135] D. P. Lathrop, J. Fineberg, and H. S. Swinney, “Turbulent flow between concentric rotating cylinders at large Reynolds numbers”, Phys. Rev. Lett. 68, 1515-1518 (1992). 
[136] H. Ji, M. Burin, E. Schartman, and J. Goodman, "Hydrodynamic turbulence cannot transport angular momentum effectively in astrophysical disks", Nature 444, 343346 (2006).

[137] D. Borrero-Echeverry, M. F. Schatz, and R. Tagg, “Transient turbulence in TaylorCouette flow", Phys. Rev. E 81, 025301 (2010).

[138] R. van Hout and J. Katz, "Measurements of mean flow and turbulence characteristics in high-Reynolds number counter-rotating Taylor-Couette flow", Phys. Fluids 23, 105102 (2011).

[139] M. Bilson and K. Bremhorst, "Direct numerical simulation of turbulent TaylorCouette flow”, J. Fluid Mech. 579, 227-270 (2007).

[140] W. He, M. Tanahashi, and T. Miyauchi, "Direct numerical simulation of turbulent Taylor-Couette flow with high Reynolds number", Advances in Turbulence XI: Proceedings of the 11th EUROMECH European Turbulence Conference held June 2528, 2007, in Porto, Portugal (2007).

[141] S. Dong, "Direct numerical simulation of turbulent Taylor-Couette flow", J. Fluid Mech. 587, 373-393 (2007).

[142] D. Pirro and M. Quadrio, "Direct numerical simulation of turbulent Taylor-Couette flow”, Eur. J. Mech. B-Fluid 27, 552-566 (2008).

[143] P. R. Fenstermacher, H. L. Swinney, and J. P. Gollub, "Dynamical instabilities and the transition to chaotic Taylor vortex flow”, J. Fluid Mech. 94, 103-128 (1979).

[144] S. Tokgoz, G. E. Elsinga, R. Delfos, and J. Westerweel, "Experimental investigation of torque scaling and coherent structures in turbulent Taylor-Couette flow", J. Phys.: Conf. Ser. 318, 082018 (2011).

[145] B. Martínez-Arias, J. Peixinho, O. Crumeyrolle, and I. Mutabazi, "Effect of the number of vortices on the torque scaling in Taylor-Couette flow", J. Fluid Mech. 748, 756-767 (2014).

[146] X. He, D. Funfschilling, H. Nobach, E. Bodenschatz, and G. Ahlers, "Transition to the ultimate state of turbulent Rayleigh-Bénard convection”, Phys. Rev. Lett. 108, 024502 (2012).

[147] P. Wei, S. Weiss, and G. Ahlers, "Multiple transitions in rotating turbulent rayleighbénard convection”, Phys. Rev. Lett. 114, 114506 (2015).

[148] S. G. Huisman, R. C. A. van der Veen, C. Sun, and D. Lohse, "Multiple states in highly turbulent Taylor-Couette flow”, Nat. Commun. 5, 3820 (2014).

[149] M. Gul, G. E. Elsinga, and J. Westerweel, "Experimental investigation of geometry on torque hysteresis behaviour of Taylor-Couette flow", 15th European Turbulence Conference held August 25-28, 2015, in Delft, The Netherlands (2015). 
[150] G. I. Taylor, "Experiments on the motion of solid bodies in rotating fluids", Proc. R. Soc. Lond. A 104, 213-218 (1923).

[151] R. Ostilla-Mónico, R. Verzicco, S. Grossmann, and D. Lohse, “The near-wall region of highly turbulent Taylor-Couette flow”, J. Fluid Mech. 788, 95-117 (2016).

[152] S. Grossmann, D. Lohse, and C. Sun, "Velocity profiles in strongly turbulent TaylorCouette flow”, Phys. Fluids 26, 025114 (2014).

[153] G. Ahlers, E. Bodenschatz, and X. He, "Logarithmic temperature profiles of turbulent Rayleigh-Bénard convection in the classical and ultimate state for a Prandtl number of 0.8”, J. Fluid Mech. 758, 436-467 (2014).

[154] E. P. van der Poel, R. Ostilla-Mónico, R. Verzicco, S. Grossmann, and D. Lohse, “Logarithmic mean temperature profiles and their connection to plume emissions in turbulent Rayleigh-Bénard convection”, Phys. Rev. Lett. 115, 154501 (2015).

[155] S. Merbold, S. Fischer, and C. Egbers, "Torque scaling in Taylor-Couette flow - an experimental investigation”, J. Phys.: Conf. Ser. 318, 082017 (2011).

[156] I. Marusic, J. P. Monty, M. Hultmark, and A. J. Smits, “On the logarithmic region in wall turbulence", J. Fluid. Mech. 716 (2013).

[157] J. Kiefer, "Sequential minimax search for a maximum”, Proc. Amer. Math. Soc. 4, 502-506 (1953). 


\section{Summary}

Turbulence, bubbles and drops are fluid phenomena that are not only encountered in daily life, but also in many industrial and technological applications. Despite our familiarity with these concepts, the understanding of them is far from complete. In this thesis, several questions related to the fields of drop impact and Taylor-Couette turbulence are answered.

A drop impacting on a surface can display many complicated phenomena, such as splashing, jetting, spreading and rebounding. In this work the focus is on the deformation of the drop just before impact, which can cause a bubble to be entrapped. For many applications, such as inkjet printing and spray painting, it is crucial to control the size of this entrapped bubble. To study the deforming drop and resulting bubble, a high-speed color interferometry method is developed to dynamically measure the air layer profile under impacting drops (chapter 1). This tool offers the advantage of providing absolute thickness values without the need for a reference, in contrast to single-wavelength interferometry. It is shown that the shape of the air layer can be accurately and reliably reconstructed from color interference patterns using the method's color-matching approach. In chapter 2 the method is used to measure the size of the entrapped bubble under impacting drops for a large range of impact velocities. These experiments and accompanying simulations and theory show a pronounced maximum in the bubble size, caused by competing inertial and capillary effects. In chapter 3 the method is applied to micropatterned surfaces, which serve as a model for the rough surfaces of many applications. It is found that the liquid-air interface globally resembles the dimple that is created in the case of smooth surfaces, but that locally symmetry-breaking occurs. In the case of impact on a dense array of micropillars, the central dimple height is increased due to an additional pressure build-up.

The second part of this thesis treats several aspects of Taylor-Couette (TC) turbulence. Turbulent flows, characterized by chaotic property changes, are notoriously hard to predict. A better understanding of the fundamentals would allow for improvement of technological and industrial processes and a better understanding of many natural phenomena. To study turbulence in a highly controlled way, the Taylor-Couette system is employed, which consists of flow between two coaxial cylinders that can independently rotate. The design and construction of a new Taylor-Couette system with precise temperature control and excellent optical 
access is treated in chapter 4 . The new facility offers the possibility to accurately study the process of boiling inside a turbulent flow.

The ability to extrapolate data from flows on the lab scale to the large scales in nature and industry is paramount. While scaling laws exist, these can be rendered impractical if there are flow transitions with increasing Reynolds number, or if multiple states can coexist at the same Reynolds number. It was expected that for highly turbulent flows, only one possible flow state could occur at a certain set of parameters. In chapter 5, however, the first evidence is reported of multiple turbulent states, characterized by stable roll structures with a different number of rolls, at very high Taylor number of roughly $10^{12}\left(\mathrm{Re}=\mathscr{O}\left(10^{6}\right)\right)$. By systematically probing the phase space of the inner and outer cylinder velocity, it is found that these states are very robust (chapter 6). It can be expected that they persist for $\mathrm{Ta}=10^{13}$ and beyond. Additionally, it is found that the rotation ratio of the cylinders is the main parameter that controls the transition. The robustness of the multiple states also shows in experiments in the aforementioned new TC-setup with a larger aspect ratio. Here, the phenomena are even more rich, and for the first time in turbulent TC flow, an antisymmetrical roll state has been observed.

In the previous chapters, TC systems with a cylinder radius ratio of approximately 0.71 were used. In the last chapter of this thesis, several flow properties in a setup with a smaller radius ratio of 0.5 are investigated with high-resolution particle image velocimetry, at a Taylor number of up to $6.2 \cdot 10^{9}$. Because of the stronger curvature, the angular velocity profiles follow a log-law more closely than the azimuthal velocity profiles. The stronger curvature also allows for investigation of the scaling of the wind Reynolds number in the classical turbulent regime, which was found to agree with the theoretically predicted 3/7-scaling. By measuring the velocity profiles and analyzing turbulent plume ejections at several axial positions, it was found that profiles with a logarithmic signature are created at plume-emitting regions, confirming results from direct numerical simulations. 


\section{Samenvatting}

Turbulentie, bellen en druppels zijn fenomenen die we niet alleen tegenkomen in het dagelijks leven, maar ook in vele industriële en technologische toepassingen. Ondanks dat we zo bekend zijn met deze concepten, is het begrip ervan verre van compleet. In dit proefschrift worden verscheidene vragen over druppelinslag en Taylor-Couette turbulentie beantwoord.

Een druppel die neerkomt op een oppervlak kan vele gecompliceerde gedragingen laten zien, zoals spatten, uitspreiden en terugspringen. Dit werk richt zich op de vervorming van de druppel net voor inslag, waarbij een bel kan worden ingevangen. Voor veel toepassingen, zoals inkjetprinten en verfspuiten, is het cruciaal om de belgrootte te kunnen beïnvloeden. Om de vervormende druppel en resulterende bel te bestuderen, is een meetmethode ontwikkeld die met behulp van hogesnelheids-kleureninterferometrie dynamisch de luchtlaag onder inslaande druppels kan meten. Deze techniek produceert absolute waarden voor de laagdikte zonder dat een referentie nodig is, een voordeel ten opzichte van interferometrie die licht van een enkele golflengte gebruikt. Met een aanpak gebaseerd op kleurcorrespondentie wordt getoond dat de vorm van de luchtlaag nauwkeurig en herhaalbaar kan worden gereconstrueerd uit de gemeten interferentiepatronen. In hoofdstuk 2 wordt de methode gebruikt om de grootte van de ingevangen bel onder inslaande druppels te meten voor een groot bereik van inslagsnelheden. Deze metingen en aansluitende simulaties en theorie laten een maximum zien in de belgrootte, veroorzaakt door elkaar tegenwerkende intertiële en capillaire effecten. In hoofdstuk 3 wordt de methode toegepast op oppervlakken met micropatronen, die dienen als model voor ruwe oppervlakken in praktijktoepassingen. Het blijkt dat de vloeistof-lucht-grenslaag globaal overeenkomt met het kuiltje in de druppel dat onstaat bij gladde oppervlakken, maar dat lokaal de symmetrie verloren gaat. In het geval van inslag op een dicht netwerk van micropilaren wordt de hoogte van het kuiltje in de druppel groter door een aanvullende drukopbouw.

Het tweede deel van dit proefschrift behandelt diverse aspecten van TaylorCouette (TC) turbulentie. Turbulente stromingen, gekarakteriseerd door chaotische veranderingen in hun eigenschappen, zijn buitengewoon moeilijk te voorspellen. Een beter basisbegrip zou het mogelijk maken om technologische en industriële processen te verbeteren en grootschalige natuurlijke fenomenen beter te begrijpen. Om turbulentie op een gecontroleerde manier te onderzoeken, wordt 
er gebruik gemaakt van het Taylor-Couette systeem, dat bestaat uit stroming tussen twee coaxiale cylinders die onafhankelijk kunnen draaien. Het ontwerp en de constructie van een nieuw Taylor-Couette systeem met precieze temperatuurregeling en goede optische toegang wordt behandeld in hoofdstuk 4. Deze nieuwe faciliteit biedt de mogelijkheid om faseovergangprocessen in een turbulente stroming nauwkeurig te bestuderen.

De mogelijkheid om data te extrapoleren van stromingen in de labschaal naar grotere schalen in de natuur en industrie is van groot belang. Alhoewel er schalingswetten bestaan, kunnen deze onpraktisch zijn als er stromingsovergangen plaatsvinden met toenemend Reynoldsgetal, of als meerdere toestanden tegelijk kunnen bestaan bij hetzelfde Reynoldsgetal. Er werd gedacht dat voor hoogturbulente stromingen er maar één mogelijke stromingstoestand kon bestaan bij een specifieke parameterset. In hoofdstuk 5, daarentegen, wordt het eerste bewijs van meerdere turbulente toestanden gegeven bij een hoog Taylorgetal van ongeveer $10^{12}\left(\operatorname{Re}=\mathscr{O}\left(10^{6}\right)\right)$. Deze turbulente toestanden worden gekarakteriseerd door stabiele rolstructuren met verschillende rolaantallen. Door het systematisch onderzoeken van de faseruimte van de binnen- en buitencylinderrotatie, is gevonden dat de toestanden zeer robuust zijn (hoofdstuk 6). De verwachting is dat ze bestaan voor $\mathrm{Ta}=10^{13}$ en hoger. Daarnaast is ontdekt dat de rotatieverhouding van de cylinders de belangrijkste parameter is die de toestandsovergangen bepaalt. De robuustheid van de meerdere toestanden laat zich ook zien in experimenten in de hiervoor genoemde nieuwe TC-opstelling met een grotere hoogtebreedteverhouding. Hierbij zijn de fenomenen nog complexer, en is er, voor de eerste keer in turbulente TC-stroming, een antisymmetrische roltoestand waargenomen.

In de voorgaande hoofdstukken zijn TC-systemen met een cylinderstraalverhouding van ongeveer 0.71 behandeld. In het laatste hoofdstuk van dit proefschrift worden verscheidene stromingseigenschappen onderzocht in een opstelling met een kleinere cylinderstraalverhouding van 0.5 , met behulp van hogeresolutie-PIV (stromingsmeting m.b.v. deeltjes), voor een Taylorgetal tot $6.2 \cdot 10^{9}$. Vanwege de sterkere kromming komen de profielen van de hoeksnelheid beter overeen met een logaritmische functie dan die van de azimutale snelheid. De sterkere kromming laat ook toe dat de schaling van het wind-Reynoldsgetal in het klassieke turbulente regime kan worden onderzocht. Deze blijkt overeen te komen met de theoretisch voorspelde 3/7-schaling. Door het meten van snelheidsprofielen en het analyseren van turbulente-pluim-emissie op meerdere axiale posities, is gebleken dat profielen met een logaritmisch kenmerk ontstaan op gebieden met turbulentepluim-emissie, wat resultaten van directe numerieke simulaties bevestigd. 


\section{Acknowledgements}

The tangible result of the four years of my $\mathrm{PhD}$ research at the Physics of Fluids group lies in front you. This could not have been possible in this manner without the help, support and collaboration of many people.

My time at the Physic of Fluids group already started with my bachelor's assignment, which I performed under the great supervision of Peichun and Chao. These people laid the foundation of my scientific progress, which was further solidified during my master's assignment supervised by Tuan and Chao. The possibilities that Detlef and Chao offered, in combination with the great atmosphere, quickly convinced me to do my $\mathrm{PhD}$ at the same group. Chao, you have been a continuous support as an extremely knowledgeable and motivating supervisor. Thank you for discussing ideas and results, working on papers together, and in general being a great person to interact with. Detlef, I learned a lot from you, and I am especially thankful for the critical, productive and inspiring environment that you create.

During my research I worked together with many people, both on turbulence and drop impact related work. On both sides the collaboration has always been very pleasant. Thank you Tuan, Wilco, Diederik, Koen, Ivo, Devaraj, Jacco, Maurice and Peichun for the great collaboration on the drop impact research. We were able to combine measurements, theory and simulations, to achieve somewhat of a 'holy grail' in science.

On the Taylor-Couette side of things, my lab mate, office mate and friend Sander has been an invaluable help, guiding me through the workings of the Taylor-Couette machine and many techniques and theories. I specifically appreciate your eye for detail and quest for extremely high quality. This has led to some excellent joint work, such as the discovery of the multiple turbulent states. I also fondly remember our 'measurement campaign' to Cottbus, Germany, where we overcame many challenges, from the van breaking down, fixing a leaking setup to consuming huge schnitzels. On a personal level we share the interests of photography, traveling and mountain biking, which was very fun to do together on many occasions.

Ruben, Rodrigo and Dennis joined the Taylor-Couette team in the second half of my PhD. It is great to see how you guys have picked it up and are expanding the TC research in many new directions. Ruben, we've had collaboration on two 
manuscripts already, and I very much appreciate your view on things from the 'engineering side'. I will miss the conferences and (Skype) meetings with Chao and the three of you! Thank you Peter and Michael, for joining us for very fruitful summer projects. To the other 'turbulence team' in the Water tunnel lab: Vivek, Varghese, Biljana and Elise, thank you for the good interactions.

The Taylor-Couette setup brings me to the technicians of our group: GertWim, Martin, Bas and recently Dennis. Jullie zijn de motor die de vakgroep doet draaien, en door het ontwerpen, bouwen en onderhouden van de opstellingen zijn jullie absoluut onmisbaar. Ik heb veel geleerd over techniek, niet alleen in de professionele samenwerking, maar ook in de onderonsjes in jullie kantoor over bijvoorbeeld auto's, motoren en landbouwapparatuur. Mijn dank gaat ook uit naar de mensen van TCO, onder andere Rindert, Sip Jan en Geert, voor jullie werk aan de opstellingen.

Also much time was spent in ME249, where I had a great time with my office mates Sander, Erwin, Rodolfo, Xiaojue, Xiumei, Pengyu and José. Many great conversations and discussions were had about a myriad of subjects; science-related and less-science-related. Sander, you would always be happy to discuss about the Mac, photography, Matlab vs. Mathematica, in addition to Taylor-Couette of course. We had many laughs and a great time, and I'm happy you'll be assisting me March 24th. Erwin, you keep me on point with your strong but thought-through views on the world, and we have become friends through the time at POF. A mention also goes out to 'honorary office mate' and friend Matthijs Neut who on many occasions borrowed my chair. All is forgiven. Rodolfo, thank you for lots of interaction and laughs. Xiaojue, you work so hard, but are always happy to talk.

During my PhD, many collaborations were had. Thanks go out to Sebastian, Uwe, Christoph and the technicians at BTU Cottbus. Also thank you Freja and Dan for the collaboration on Rayleigh-stable TC. Thank you Agathe Chiouppe for sharing data with us. Thank you Xuehua and Erik for the project on the nanodroplets.

The POF group has a great atmosphere due to the diverse group of people. Thank you Rianne, Enrique, Pascal, Erik-Jan, Sander W., Claas-Willem, Vamsi, Maarten, Marise, Michiel, Matthijs, Oscar, Álvaro, Tim, Minori, Alexander, Loreto, Ivan, Shantanu, Yantao and all of the others for a great time at POF! Thank you Joost, Wilco, Rianne and Enrique for the fun and educational time we had as teaching assistants together for the course Fysica van Vloeistoffen of Devaraj. Joanita, bedankt voor je absoluut onmisbare bijdrage aan de vakgroep. Aram, geen lid van POF maar toch een vaakgeziene gast, bedankt voor de steun en super dat je me assisteert als paranimf!

$\mathrm{Nu}$ weer een stapje terug in de tijd, toen ik op de middelbare school de eerste lessen natuurkunde van Benno kreeg. Door het enthousiasme en de kunde die je in het lesgeven stopte, en de interesse voor natuurkunde die je daarmee wekte, was de keus voor een natuurkundestudie niet moeilijk. Daarmee heb je een grote 
rol gespeeld in dit uiteindelijke resultaat. Bedankt!

Tijdens deze studie heb ik ook een fantastische tijd gehad, onder andere bij het afstuderen met Gerben, Oscar, Nikolai en Matthias. Deze en andere vrienden, bedankt voor de steun toentertijd en tijdens mijn promotie!

Dit dankwoord komt bijna tot het einde, maar er zijn nog een paar belangrijke mensen die ik wil bedanken. Ackelien, je bent een ongelofelijke steun. Ontzettend bedankt dat je me zo hebt bijgestaan, van de kleine tot de grote dingen. En mijn ouders, die altijd voor me klaar staan en er altijd voor gezorgd hebben dat niets in de weg stond om dit resultaat te bereiken. Dank jullie wel! 


\section{About the author}

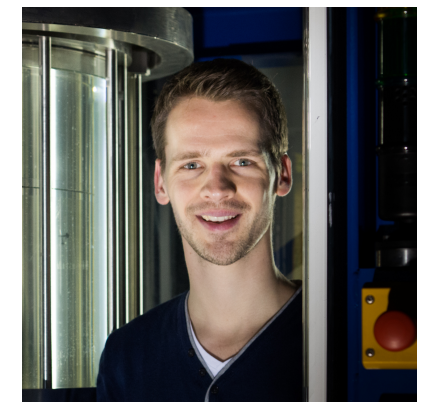

Roeland Cornelis Adriaan van der Veen was born in Enschede, The Netherlands on August 10th, 1988. He attended the 'Bonhoeffer College' in Enschede where he received his high school diploma in 2006. He studied Applied Physics at the University of Twente, obtaining his Bachelor's degree in 2009 and doing his internship at the University of Chicago in 2010, studying Leidenfrost dynamics. In 2011, he received his Master's degree with honors for developing a drop impact visualization method at the Physics of Fluids group. He was awarded a University of Twente Master Thesis Prize and a KHMW Shell Master Thesis Prize for this work. His PhD at the same group involved the continuation of drop impact studies and the new subject of Taylor-Couette turbulence. Apart from physics he takes an interest in technology, photography, traveling, cycling, and many other things. 


\section{Publications}

How micropatterns and air pressure affect splashing on surfaces

P. Tsai, R. C. A. van der Veen, M. van de Raa and D. Lohse

Langmuir 26, 16090-16095 (2010)

Direct measurements of air layer profiles under impacting droplets using highspeed color interferometry

R. C. A. van der Veen, T. Tran, D. Lohse and C. Sun

Phys. Rev. E 85, 026315 (2012)

Geometry of the vapor layer under a Leidenfrost drop

J. C. Burton, A. L. Sharpe, R. C. A. van der Veen, A. Franco and S. R. Nagel

Phys. Rev. Lett. 109, 074301 (2012)

Maximal air bubble entrainment at liquid-drop impact

W. Bouwhuis, R. C. A. van der Veen, T. Tran, D. L. Keij, K. G. Winkels, I. R. Peters,

D. van der Meer, C. Sun, J. H. Snoeijer and D. Lohse

Phys. Rev. Lett. 109, 264501 (2012)

Multiple states in highly turbulent Taylor-Couette flow

S. G. Huisman, R. C. A. van der Veen, C. Sun and D. Lohse

Nat. Commun. 5, 3820 (2014)

How microstructures affect air film dynamics prior to drop impact

R. C. A. van der Veen, M. H. W. Hendrix, T. Tran, C. Sun, P. A. Tsai and D. Lohse Soft Matter 10, 3703-3707 (2014) 
Mixed mode of dissolving immersed nanodroplets at a solid-water interface

X. Zhang, J. Wang, L. Bao, E. Dietrich, R. C. A. van der Veen, S. Peng, J. Friend, H. J. W. Zandvliet, L. Yeo and D. Lohse

Soft Matter 11, 1889-1900 (2015)

The boiling Twente Taylor-Couette (BTTC) facility: Temperature controlled turbulent flow between independently rotating, coaxial cylinders

S. G. Huisman, R. C. A. van der Veen, G.-W. H. Bruggert, D. Lohse and C. Sun Rev. Sci. Inst. 6, 065108 (2015)

Azimuthal velocity profiles in Rayleigh-stable Taylor-Couette flow and implied axial angular momentum transport

F. Nordsiek, S. G. Huisman, R. C. A. van der Veen, C. Sun, D. Lohse and D. P. Lathrop J. Fluid Mech. 774, 342-362 (2015)

Taylor-Couette turbulence at radius ratio $\eta=0.5$ : scaling, flow structures and plumes

R. C. A. van der Veen, S. G. Huisman, S. Merbold, U. Harlander, C. Egbers, D. Lohse and C. Sun

submitted

Universal decay of high Reynolds number Taylor-Couette turbulence

R. A. Verschoof, S. G. Huisman, R. C. A. van der Veen, C. Sun and D. Lohse submitted

Exploring the phase space of multiple states in highly turbulent Taylor-Couette flow

R. C. A. van der Veen, S. G. Huisman, O.-Y. Dung, H. L. Tang, C. Sun and D. Lohse submitted 




\section{Propositions}

belonging to the dissertation

\section{Turbulence, bubbles and drops}

Roeland C. A. van der Veen, March 24, 2016

1. Color interferometry is a useful technique to investigate very thin air layers below impacting drops.

Chapter 1, 2 and 3 of this thesis

2. Multiple stable states in highly turbulent Taylor-Couette flow exist far into the ultimate turbulent regime and are very reproducible.

Chapter 5 and 6 of this thesis

3. Despite the many problems with the current state of scientific research, science is and will be the only way to truly understand nature.

4. Dissecting natural phenomena in a scientific way only enhances their beauty.

5. Space colonization can ensure a long-term future for humans and by attempting it we can likely solve the Fermi paradox.

6. The value of browsing Wikipedia as both a recreational and professional activity is still being underestimated.

VII. Consistency is key. 


\section{Stellingen}

behorende bij het proefschrift

\section{Turbulentie, bellen en druppels}

Roeland C. A. van der Veen, 24 maart 2016

1. Kleureninterferometrie is een goede techniek om de zeer dunne luchtlagen onder inslaande druppels te onderzoeken.

Hoofdstuk 1, 2 en 3 van dit proefschrift

2. Meerdere stabiele toestanden in hoogturbulente Taylor-Couette-stroming bestaan tot ver in het ultieme turbulente stromingsregime en zijn zeer reproduceerbaar.

\section{Hoofdstuk 5 en 6 van dit proefschrift}

3. Ondanks de vele problemen in de uitvoering van huidig wetenschappelijk onderzoek, is en blijft wetenschap de enige manier om de natuur echt te begrijpen.

4. Het ontleden van natuurverschijnselen op een wetenschappelijke manier vergroot hun schoonheid enkel.

5. Ruimtekolonisatie kan een langetermijnbestaan van de mensheid garanderen, en door een poging te wagen kan de Fermi-paradox opgelost worden.

6. De waarde van het lezen van Wikipedia als zowel een hobby als een beroepsmatige activiteit wordt nog steeds onderschat.

VII. Consistent zijn is uitermate belangrijk. 

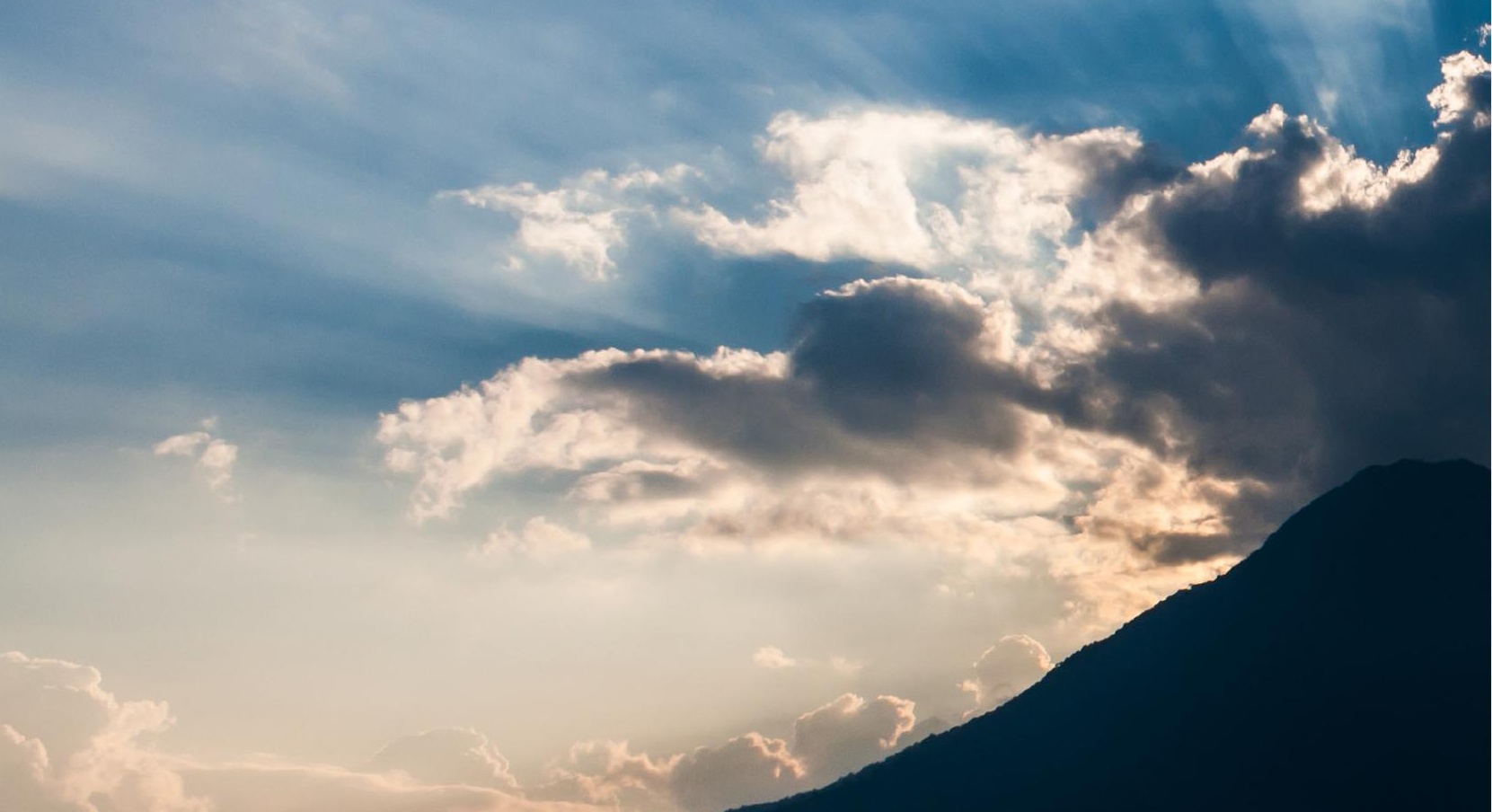

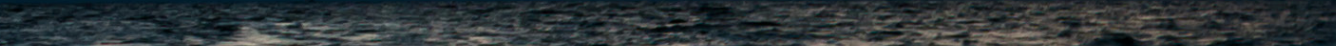

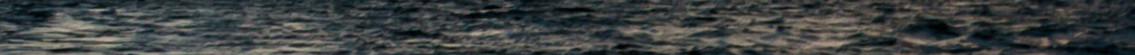
In 